\author{
STELLAR CHROMOSPHERES : \\ H-ALPHA AND Ca II K PROFILES
}

\author{
BY \\ DOMINIC MICHAEL ZARRO \\ A THESIS SUBMITTED TO THE AUSTRALIAN NATIONAL UNIVERSITY \\ FOR THE DEGREE OF DOCTOR OF PHILOSOPHY \\ MT. STROMLO AND SIDING SPRINGS OBSERVATORIES \\ RESEARCH SCHOOL OF PHYSICAL SCIENCES \\ AUSTRALIAN NATIONAL UNIVERSITY \\ CANBERRA, AUSTRALIA
}

MAY, 1983 
Unless otherwise acknowledged in the text, the work reported in this thesis was performed by the candidate.

\section{D) Zano}

Dominic Michael Zarro

May, 1983 


\section{ACKNOWLEDGEMENTS}

It is with great pleasure that I acknowledge the encouragement, insight, expertise and, above all, friendship offered to me by my supervisor Dr. Alex Rodgers throughout all phases of this Ph.D dissertation.

Deep appreciation is also due to many people who have collectively and individually assisted me during the development of this thesis. They include:

- Drs Tom Ayres, Jeff Linsky, Kjell Eriksson and Bob Stencel for numerous useful discussions concerning stellar chromospheres and radiative transfer theory;

- the Mt. Stromlo and Siding Spring Observatories Electronic, Mechanical and Optical workshop staff for their expert maintenance of the telescopes and data acquisition systems employed for this investigation;

- the Vax, Univac and Cray computing staff at Mt. Stromlo, A.N.U and N.C.A.R (Boulder), respectively, for their provision of facilities so vital to the empirical and theoretical aspects of this thesis;

- the Mt. Stromlo publications and photographics sections for their assistance with various diagrams appearing in the ensuing manuscript;

- the Mt. Stromlo faculty, administrative and student bodies for providing a stimulating working environment: 
and

- last, but not least, my wife Kin for her constant emotional (and financial) support during the course of this research.

Finally, I acknowledge the financial support of a Commonwealth Postgraduate Research award during 1979-83, and the International Astronomical Union for funding generously my travel expenses to visit the Joint Institute for Laboratory Astrophysics at Boulder, Colorado. 


\section{$\underline{\text { ABSTRACT }}$}

An atlas of high resolution $\mathrm{H}-\mathrm{alpha}$ and $\mathrm{Ca}$ II $\mathrm{K}$ Echelle spectra is presented for 85 predominantly southern stars, covering a wide range of spectral type and luminosity class. This homogenous data set constitutes a valuable foundation upon which to investigate the capacity of the H-alpha profile as a chromospheric diagnostic in late type stars. A coordinated analysis of $\mathrm{H}$-alpha and $\mathrm{Ca}$ II $\mathrm{K}$ profiles has yielded the following results :

(i) A non-linear, spectral-type dependent correlation of increasing $\mathrm{H}-\mathrm{alpha}$ full width at half depth with increasing Ca II K Wilson-Bappu width. This behaviour concurs with that found previously by Kraft et al. (1964).

(ii) For many stars, Ca II K Wilson-Bappu widths - when compared with upper limit non-thermal broadening velocities, deduced approximately from H-alpha widths - are found to exceed the limiting Doppler core width of the $\mathrm{K}$ line absorption coefficient. This result suggests that damping effects may be an important consideration in interpretations of the Wilson-Bappu effect.

(iii) A correspondence in asymmetry morphology between the $\mathrm{H}-\mathrm{alpha}$ absorption and $\mathrm{Ca}$ II $\mathrm{K}$ emission cores in late type giants and supergiants. In particular, red-dominated $\mathrm{Ca}$ II $\mathrm{K}$ asymmetry is reflected in redward asymmetry in the corresponding $\mathrm{H}$-alpha core. More striking, is an apparent 
one-to-one mapping between fine structures present within the cores of these lines. The correlation highlights the importance of the H-alpha profile as a tracer for chromospheric velocity fields in late type stars.

(iv) A correlation of increasing $\mathrm{H}$-alpha central residual flux with increasing $\mathrm{Ca}$ II $\mathrm{K}$ emission strength among $\mathrm{G}$ and $\mathrm{K}$ dwarfs. This result suggests that the H-alpha core may provide a viable diagnostic of chromospheric activity in cool main sequence stars.

Mathematical and computational details of the Auer and Mihalas (1969) Complete Linearisation code are discussed. The non-LTE radiative transfer code is applied to a study of $\mathrm{H}-\mathrm{alpha}$ and $\mathrm{Ca}$ II $\mathrm{K}$ formation in schematic dwarf chromosphere models. Numerical simulations indicate that the H-alpha core brightening phenomenon, observed in active dwarf stars, can be understood in terms of enhanced chromospheric temperatures and electron densities in magnetic active regions on their surfaces, similar to solar plages.

Finally, a computational study of $\mathrm{H}-\mathrm{alpha}$ in 70 Ophiuchi $\mathrm{A}$ and Arcturus is performed in an endeavour to establish the usefulness of the H-alpha line as a consistency check on chromospheric models inferred from $\mathrm{Ca}$ II and Mg II diagnostics. 
page

TITLE

STATEMENT

ACKNOWLEDGEMENT

ABSTRACT

CHAPTER ONE : INTRODUCTION

1.1 GENERAL INTRODUCTION

1.2 WIDTH-LUMINOSITY CORRELATIONS

1.2.1 Interpretations of the Wilson-Bappu Effect

1.3 H-ALPHA AS A CHROMOSPHERIC DIAGNOSTIC

1.4

OUTLINE OF THESIS

CHAPTER TWO : ATLAS OF H-ALPHA AND Ca II $\mathrm{K}$ OBSERVATIONS

2.1 INTRODUCTION

2.2

INSTRUMENTATION AND OBSERVATIONAL PROCEDURE

2.3

REDUCTIONS

APPENDIX

CHAPTER THREE : ANALYSIS OF H-ALPHA AND $\mathrm{Ca}$ II $\mathrm{K}$ PROFILES

3.2 H-ALPHA AND Ca II K LINE PARAMETERS

3.2.1 Definitions of Quantities

3.2.2 Discussion of Errors

3.3 H-ALPHA CORE INTENSITY AND CHROMOSPHERIC ACTIVITY 31

3.4 COMPARISON OF H-ALPHA AND Ca II K LINE WIDTHS 35

3.4.1 The Empirical Ho-Wo Relation 35

3.4.2 The Role of Chromospheric Microturbulence in 37

$\mathrm{H}-\mathrm{Al}$ pha and $\mathrm{Ca}$ II $\mathrm{K}$ Width-luminosity Correlations

3.5 COMPARISON OF H-ALPHA AND Ca II K PROFILE ASYMMETRIES 
page

CHAPTER FOUR : DESCRIPTION OF THE PROFILE SYNTHESIS CODE 48

$\begin{array}{lll}4.1 & \text { INTRODUCTION } & 48\end{array}$

4.2 MODEL ATMOSPHERE 49

4.3 MODEL ATOMS 50

4.4 CALCULATION OF LTE ATOMIC POPULATIONS

4.5 CONTINUUM AND LINE OPACITY CALCULATIONS

4.5.1 Continuum Opacity Sources 54

4.5.2 Line Opacities 59

4.6 FEAUTRIER SOLUTION OF RADIATIVE TRANSFER EQUATION 63

$\begin{array}{lllll}4.7 & \text { FORMULATION OF } & \text { THE } & \text { RATE EQUATIONS }\end{array}$

4.7.1 Radiative Rates 67

4.7.2 Collisional Rates 69

4.7.3 The Equations of Statistical Equilibrium 70

4.8 CALCULATION OF NON-LTE ATOMIC POPULATIONS

4.8.1 Lamda Iteration 72

4.8.2 The Complete Linearisation Method 73

4.8.3 Hydrostatic Equilibrium Adjustment of Hydrogen 76 And Electron Number Densities

4.9 OVERVIEW OF RADIATIVE TRANSFER CALCULATION 77

CHAPTER FIVE : THE DEPENDENCE OF H-ALPHA ON CHROMOSPHERIC 80 ACTIVITY IN MAIN SEQUENCE STARS

5.1 INTRODUCTION 80

5.2 MODEL CALCULATIONS 82

5.3 ANALYSIS OF RESULTS 86

5.4 DISCUSSION 91

5.5 CONCLUSIONS 93 
CHAPTER SIX : THE H-ALPHA PROFILE IN 70 OPHIUCHI A (KO V) 97 AND ARCTURUS (K2 I I I )

6.1 INTRODUCTION

6.2 70 OPHIUCHI A CHROMOSPHERE MODELS

6.2.1 Description of Models

6.2.2 Model Calculations and Results

6.2 .3

Discussion

107

6.3 ARCTURUS CHROMOSPHERE MODELS

110

6.3.1 Description of Models

6.3.2 Model Calculations and Results

6.3.3 Discussion

6.4 CONCLUSIONS

115

CHAPTER SEVEN : THESIS SUMMARY AND FUTURE WORK 


\subsection{GENERAL INTRODUCTION}

The H-alpha absorption profile in late type stars was investigated by Kraft et al. (1964), who discovered that its absorption full width at half depth (Ho) increased with increasing stellar luminosity. This correlation suggested that the H-alpha line could be a viable tool for stellar distance measurement and complemented the well established Wilson-Bappu effect in the $\mathrm{Ca}$ II $\mathrm{H}$ and $\mathrm{K}$ resonance lines (Wilson and Bappu, 1957).

The observed H-alpha profiles clearly behaved in a manner different from that expected of a Stark broadened line formed under LTE* in a cool stellar photosphere dominated by $\mathrm{H}^{-}$continuous opacity. In this latter circumstance, first order variations of a line width which decreases with photospheric effective temperature and wich is independent of stellar surface gravity should be observed (Gray, 1976). Kraft et al. (1964) showed this not to be the case.

Other studies of $\mathrm{H}$-alpha in late type stars have been undertaken by Reimers (1973) and Fosbury (1973). The thrust of their investigations was the relation between thermal and non-thermal chromospheric velocities deduced

* local thermodynamic equilibrium 
from $\mathrm{H}-\mathrm{al}$ pha widths and the supposed non-thermal velocity broadening of $\mathrm{Ca}$ II $\mathrm{K}$ emission. In a series of papers on H-alpha profiles in cepheid variables, Rodgers and Bell (1968, and references cited therein) demonstrated that while an LTE profile could accurately fit the wings of the H-alpha lines, the line cores were significantly wider and deeper compared to LTE predictions and displayed different kinematics to the photospheric pulsation. Thus, in these early papers there is the implication of a chromospheric component of the $\mathrm{H}-\mathrm{alpha}$ line core, which in the cooler giants and supergiants completely dominates its photospheric counterpart.

The existence of a predominant chromospheric component in the H-alpha profiles of late type stars is further supported by observations of profile asymmetries. Investigations of a ori (Weymann, 1962; Goldberg, 1979); of $\mathrm{G}$ and $\mathrm{K}$ supergiants (Mallik, 1982); of M giants (Boesgaard and Hagen, 1979) and of globular cluster red giants (Cohen, 1976; Mallia and Pagel, 1978; Cacciari and Freeman, 1981) have provided evidence for a chromospheric component of the $\mathrm{H}$-alpha profile which is characterised by large scale velocities, symptomatic of a stellar wind. In the latter context, the red dominated asymmetries observed in Ca II K (Stencel, 1978) and Mg II K (Stencel and Mullan, 1980) together with the often blue-shifted Ca II $K$ central absorptions (denoted as $K 3$ and K4) (Deutsch, 1956, 1960; Reimers, 1975, 1977), have commonly been used as signatures of outward mass fluxes and circumstellar envelopes in cool giants and supergiants. 
The above examples collectively illustrate the importance of the H-alpha profile as a diagnostic of chromospheric velocity fields. In view of the dynamical information also implicit in the Ca II K line, a coordinated study of these two spectral features can lead, therefore, to a better understanding of the physical processes occurring in stellar chromospheres. Accordingly, the broad aim of this thesis is to present a set of medium to high resolution observations of $\mathrm{H}$-alpha and $\mathrm{Ca} I I \mathrm{~K}$ in a large sample of late type stars in order to examine the systematics of $\mathrm{H}-a l p h a$ absorption profiles, and to determine empirically the extent to which velocity-related features observed therein are reflected in the chromospheric components of Ca II $\mathrm{K}$. Prior to outlining the more specific aims of this thesis, it is instructive to review briefly the nature of width-luminosity correlations obeyed by various spectral features, as chromospheric velocity fields appear to play an important underlying role in the behaviour of these correlations.

\subsection{WIDTH-LUMINOSITY CORRELATIONS}

The most notable of width-luminosity (W-L) correlations is that displayed by the chromospheric emissions seen in the cores of $\mathrm{Ca}$ II $\mathrm{H}$ and $\mathrm{K}$ in stars cooler than F5. The broadening of these lines with increasing luminosity was recognised initially by Delandres (1921) from comparisons of prismatic spectra of late type dwarfs and giants. 
Wilson and Bappu (1957) and Wilson (1959, 1967, 1976) later quantified this discovery into the famous Wilson-Bappu (W-B) effect; a remarkably tight correlation between the $\mathrm{Ca}$ II $\mathrm{K}$ full width at half maximum (FWHM, Wo) and the stellar absolute visual magnitude (Mv), which is embodied in the expression,

$$
M v=27.59-14.94 \log (\text { Wo, } \mathrm{km} / \mathrm{s})
$$

The power of this equation in determining stellar distances derives from its validity over a range of 15 magnitudes absolute, independently of spectral type although, weak dependencies on metallicity $[\mathrm{Fe} / \mathrm{H}]$ and core emission strength have been suggested by Lutz and Pagel (1978, 1982) and Glebocki and Stawikowski (1978), respectively.

Since the pioneering work of Wilson and Bappu, analogous $\mathrm{W}-\mathrm{L}$ correlations have been observed in the $\mathrm{Mg}$ II $h$ and $k$ emission cores (Moos et al., 1974; McClintock et al., 1975; Kondo et al., 1976; Weiler and Oegerle, 1979 ) and in Ly-alpha emission (MCClintock et al., 1975; Dupree, 1976). In addition, different features within the $K$ line have been shown to broaden with increasing stellar luminosity. These include the entire damping wings of Ca II K (Lutz et al., 1973), the base emission width measured at Kl (W1) (Ayres et al., 1975; Engvold and Rygh, 1978) and the separation of the twin K2 emission peaks (W2) (Cram et al., 1979).

As mentioned in the previous section, the H-alpha line in late type stars also demonstrates a 
width-luminosity relation (Kraft et al., 1964; Reimers, 1973; Fosbury, 1973). However, the proposed relationship between Ho and Mv displayed a marked scatter and a dependence upon spectral type which hindered its practical application as an accurate luminosity discriminant. Using a small sample of 15 stars, L Presto (1971) improved the correlation slightly by defining widths measured from Gaussian profiles fitted to the Doppler core of the H-alpha lines.

\subsubsection{Interpretations of The Wilson-Bappu Effect}

In their efforts to unravel the origin of the $W-B$ effect, various authors have evoked statistical mass-luminosity (Neckel, 1974) and mass-radius (Reimers, 1973 ) relations to re-express equation 1.1 in terms of the fundamental stellar parameters: surface gravity (g) and effective temperature (Teff). Generally, their results assume the form,

$$
\begin{aligned}
& w_{0} \sim g^{\alpha} T_{\text {eff }}^{\beta} \\
& \begin{aligned}
\text { with }(\alpha, \beta) & =(-.20,1.1) \text { Reimers (1973) } \\
& =(-.22,1.2) \text { Neckel (1974) }
\end{aligned}
\end{aligned}
$$

Equation 1.2 emphasises the dominance of surface gravity, over Teff, in the control of the $W-B$ effect. For example, in the region of the $H-R$ diagram where $C a$ II $K$ emission is prominent, Teff varies by at most a factor of 3. whereas the range of $g$ is five orders of magnitude between faint dwarfs and luminous supergiants. 
Despite the large amount of work invested in studies of the $W-B$ effect, there is still considerable debate over the nature of the physical mechanisms governing the observed correlation. Two schools of thought are displayed in the literature:

\section{(i) The Doppler Hypothesis}

The primary assumption of this theory is that wo is formed within the Doppler core of the Ca II $K$ line absorption profile and is, therefore, mainly a function of the Doppler broadening due to chromospheric gas motions (Athay and Skumanich, 1968; Linsky and Avrett, $1970)$.

Early attempts at reconciling this scenario with observations hinged upon the derivation of theoretical relationships between wo and the amplitude of non-thermal motions generated in the sub-photospheric convection zone (Goldberg, 1957; Hoyle and Wilson, 1958; Schatzman, 1958; Kraft, 1959). Scharmer (1976) postulated the existence of a supersonic turbulence in giant and supergiant chromospheres, and employed energy and momentum conservation arguments to deduce a gravity dependence for Wo similar to equation 1.2. More recently, Lutz and Pagel (1978) adopted the acoustic wave model of Edmunds (1978), together with a slab approximation for the Ca II $\mathrm{K}$ line formation region, to infer the power law,

$$
W_{0} \sim g^{-0.2} T_{e f f} 1.6
$$

which was in striking agreement with their empirical 
result derived from wo measurements of 55 late type stars.

These examples illustrate how the Doppler hypothesis for wo can produce, to first order, theoretical relations that mimic the $\mathrm{W}-\mathrm{B}$ effect. Curve of growth studies by Bonsack and Culver (1966) have indicated that the photospheric microturbulence deduced from weak lines in G, $K$ and $M$ stars, increased with increasing stellar luminosity and was, in fact, correlated with wo. If chromospheric small-scale motions are indeed extensions of those existing in the photosphere, then the latter result offers corroborative evidence for the Doppler theory.

The above microturbulent velocity is a largely adhoc parameter ascribed to the presence of random gas motions caused, for example, by various types of waves such as gravity, acoustic, magnetohydrodynamic or shocks. These motions are usually presumed to exist on scales much smaller than a photon mean free path (Edmunds, 1978), and manifest as a non-thermal contribution to the Doppler broadening of spectral lines.

\section{(ii) The Damping Hypothesis}

An alternative physical interpretation of the $\mathrm{W}-\mathrm{B}$ effect relies upon the assumption that optically thick $\mathrm{Ca}$ II $\mathrm{K}$ features are formed in the Lorentzian damping wing of the line absorption profile. Studies by de Jager (1958), Avrett (1973), Engvold and Rygh (1978) and Peytremann (1978) have demonstrated that under this 
circumstance, characteristic widths of Ca II K are primarily sensitive to chromospheric mass column densities, and relatively insensitive to microturbulent velocities.

The assumption of Lorentzian control of Wl (Ayres et al., 1975), together with the approximation that $\mathrm{Kl}$ is formed in the region of the temperature minimum ( $\mathrm{Tmin}$ ) (Thomas, 1973; Linsky and Ayres, 1973), resulted in the following simple relationship between $W 1$ and the mass column density $\left(\mathrm{m}^{\star}\right.$ ) at Tmin (Cram and Ulmschneider, 1978),

$$
W_{1}[\AA]=2.5\left(\mathrm{~m}^{\star}\right) 1 / 2
$$

With a similar damping assumption for W2, Ayres (1979) used plasma cooling arguments to derive scaling laws of the forms,

$$
\begin{aligned}
& W_{1} \sim g^{-1 / 4} \mathrm{~T}_{\text {eff }}{ }^{7 / 4} \\
& W_{2} \sim g^{-1 / 4} \mathrm{~T}_{\text {eff }}-5 / 4 \Delta \lambda_{D}{ }^{1 / 2} \\
& \text { where } \Delta \lambda_{D}=\text { the Doppler width }
\end{aligned}
$$

The common gravity behaviour of $w 1$ and $w 2$ led to the postulate that every width between the $\mathrm{K} 1$ and $\mathrm{K} 2$ features, in particular wo, ought to scale in the same fashion. Most importantly, it has been suggested that the luminosity broadening of damping controlled Ca II $K$ features can best be described as a response to the "thickening" of stellar chromospheres with decreasing surface gravity, rather than an increase in the amplitude 
of non-thermal motions (Ayres, 1979).

Supportive evidence for the Damping hypothesis was provided by the success of partial redistribution (PRD) calculations in matching the observed limb darkening of solar $\mathrm{Kl}$ and $\mathrm{K} 2$ features (Zirker, 1968; Shine et al.., 1975). These observations indicated near coherent scattering (and hence little Doppler redistribution) at and beyond the $\mathrm{K} 2$ emission peaks.

Finally, these PRD studies bring us to the important conclusions of Basri (1979) concerning interpretations of W-L correlations. From prototype PRD calculations of $\mathrm{Ca}$ II and Mg II lines in supergiant chromospheres, Basri (1979) demonstrated that, despite the Kl feature being formed in the line damping wing, Doppler motions can critically influence its wavelength location by controlling the frequency diffusion of core photons into the line wing. Such effects are expected to be particularly severe in luminous giants where low atmospheric densities lead to very small collisional redistribution rates and a high degree of coherent scattering. Based on similar PRD Ca II K computations, Severino (1982) recently questioned the assumption that $\mathrm{Kl}$ is formed at the temperature minimum, and has re-affirmed the importance of Doppler motions in affecting characteristic widths of optically thick Ca II K features. 


\subsection{H-ALPHA AS A CHROMOSPHERIC DIAGNOSTIC}

The study of $W-L$ correlations, in particular the $W-B$ effect, has stimulated considerable advances in the development of the Ca II (and Mg II) lines as chromospheric diagnostics. Extensive observations of these spectral features, in conjunction with detailed numerical transfer calculations, have led to significant progress in the understanding of stellar chromospheres in late type stars (see Linsky, 1977, 1980; Ulmschneider, 1979 for detailed reviews).

In contrast to the large body of work involving the above resonance lines, relatively little attention has been focussed on observations and non-LTE calculations of the $\mathrm{H}$-alpha line as a means of investigating stellar chromospheres. Nevertheless, the potential usefulness of H-alpha as a chromospheric diagnostic is clearly evident in the following examples.

(a) Analyses of $\mathrm{H}-\mathrm{al}$ pha formation in young $\mathrm{T}$-Tauri stars have drawn attention to the possibility of H-alpha emission being produced under the assumption of a photoionisation dominated source function, and have indicated the importance of chromospheric velocity gradients in achieving consistency between theory and observations (Dumont et al., 1973; Thomas, 1980).

(b) Based on non-LTE calculations of H-alpha profiles in the metal-weak giants Arcturus and HD 122563, Eriksson 
(1980) concluded that, in addition to a chromospheric temperature rise, a microturbulent velocity field increasing with height in the chromosphere was required to obtain agreement with observed line widths and strengths.

(c) Cram and Mullan (1979) and Cram and Woods (1982) have explored the feasibility of $\mathrm{H}$-alpha emission profiles in dMe stars as indicators of chromospheric thermal structure and optical thickness under conditions of enhanced non-radiative heating.

(d) Mullan and Cram (1982) examined the time-varying H-alpha absorption line of the RS CVn system, $\lambda$ And. On the basis of comparisons with computed $\mathrm{H}-\mathrm{al}$ pha profiles, they attempted to discriminate between high transition zone pressure chromosphere models with zero non-thermal broadening and low pressure models with large macroturbuience.

In the light of these examples, and in view of the importance of the H-alpha profile as a velocity field indicator, this thesis aims to pursue and extend the applicability of H-alpha profiles in the diagnosis of late type stellar chromospheres. The adopted approach and the particular cases examined are summarised in the following section. 
1.4 OUTLINE OF THESIS

Chapter Two - An atlas of medium to high resolution

$\mathrm{H}-\mathrm{al}$ pha and $\mathrm{Ca}$ II $\mathrm{K}$ spectra is presented for a variety of

late type dwarfs, giants and supergiants later than F8.

The observational procedures employed in their

acquisition and the data reduction methods used in their

processing are described.

Chapter Three - A qualitative and quantitative analysis of the data is performed with emphasis on comparisons of H-alpha and $\mathrm{Ca}$ II $\mathrm{K}$ line widths, strengths and shapes. In particular, the relationship between the $\mathrm{H}-a l$ pha core intensity and the Ca II K emission strength is examined in order to seek a possible dependency upon chromospheric activity. The correlation between Ho and wo is investigated and sources of anomalous behaviour are noted.

Chapter Four - Mathematical and computational details of of the non-LTE radiative transfer code used in this thesis are discussed. The original version of the computer code was kindly provided by the staff at the National Center for Atmospheric Research (N.C.A.R) at Boulder, and implemented by the author on the Australian National University (A.N.U), Univac 1100.

Chapter Five - The non-LTE transfer code is utilised to compute $\mathrm{H}$-alpha and $\mathrm{Ca}$ II K profiles from schematic dwarf chromosphere models and perturbations thereof. The 
dependence of the $\mathrm{H}$-alpha line on chromospheric activity, as parameterised by enhanced chromospheric temperatures and densities, is examined.

Chapter Six - The concepts developed in the previous chapter are expanded in a study of the chromospheres of 7øOPHA (an active $\mathrm{K} \emptyset$ dwarf) and Arcturus (a metal weak K2 giant). H-alpha profiles are computed for these stars, using published chromosphere models inferred from $\mathrm{Ca}$ II $\mathrm{K}$ line analyses, and are compared with observations. In the light of these comparisons, we discuss appropriate modifications to these chromosphere models.

Chapter Seven - The conclusions from our empirical and theoretical studies of $\mathrm{H}-\mathrm{al}$ pha and $\mathrm{Ca}$ II $\mathrm{K}$ are summarised and suggestions for future work outlined. 


\section{CHAPTER TWO}

\section{ATLAS OF H-ALPHA AND CA II $K$ OBSERVATIONS}

\subsection{INTRODUCTI ON}

We present high resolution echelle spectra of the cores and wings of $\mathrm{H}-\mathrm{al}$ pha and $\mathrm{Ca}$ II $\mathrm{K}$ in 85 predominantly southern stars, covering a wide range of spectral type and luminosity class. The observations were detected and recorded with a photon counting detector, and processed using identical reduction procedures.

The purpose of these observations is to provide a homogenous data base with which to study the systematic behaviours of $\mathrm{H}-\mathrm{al}$ pha and $\mathrm{Ca}$ II $\mathrm{K}$ profiles in late type stars and, in particular, illustrate the usefulness of H-alpha as an indicator, inter alia, of velocity fields in stellar chromospheres. The latter demonstration is effected by plotting paired $\mathrm{H}$-alpha and $\mathrm{Ca}$ II $\mathrm{K}$ spectra on common photospheric velocity scales so as to highlight correlating features that may arise from chromospheric - related phenomena.

In the following sections we describe the observational and data reduction techniques employed in this thesis. The atlas of line profiles is displayed in section 2.4 and a quantitative analysis of the data will be undertaken in Chapter Three. 


\subsection{INSTRUMENTATION AND OBSERVATIONAL PROCEDURE}

H-alpha observations were obtained with an echelle spectrograph ( 79 grooves/mm grating, 63.43 blaze angle) positioned at the $\mathrm{f} / 31.7$ coude focus of the Mt. Stromlo $1.88 \mathrm{~m}$ (74 inches) telescope. The echelle grating was used in conjunction with a $5.6 \mathrm{~m}$ focal length collimator and $813 \mathrm{~mm}$ off-axis Schimdt camera, giving a dispersion of 4.Ø $\AA / \mathrm{mm}$ at $6562 \AA$ in the 34 th echelle order, and a spectral range of $51 \AA$. A cross-disperser grating ( 300 grooves $/ \mathrm{mm}, 4.9^{\circ} \mathrm{blaze}$ ) was operated in first order mode to provide 5 separate orders in an unvignetted field. Prior to December 1981, the H-alpha spectra were detected with the Mt. Stromlo one dimensional Photon Counting Array (PCA) (Stapinski et al., 1978). The detector system comprised an $\mathbf{S} 2 \emptyset$ photo-cathode and a series of 6 electrostatically focussed, fibreoptically coupled Varian intensifier tubes, imaging onto two parallel reticon diode arrays of length $12.8 \mathrm{~mm}$ and $2.5 \mathrm{~mm}$ separation. The arrays consisted of 512 diodes of 25 microns width, each double binned by event centering into 12.5 microns resolution elements. During an observation, the stellar spectrum was focussed onto one array (adjusted parallel to the direction of dispersion), whilst the sky background was monitored on the second reticon.

H-alpha data obtained subsequent to the above date were recorded with a two dimensional PCA (Stapinski et al., 1981) functioning with an ITT dual-stage 
microchannel plate (MCP) intensifier, fibreoptically coupled to a single stage electrostatic focus tube. The intensified photo events were detected by a Fairchild charge coupled device (CCD) consisting of 488 horizontal rows of 380 vertical columns of charge-coupled photosites, each measuring 12 by 18 microns. The latter were situated on 18 by $3 \emptyset$ microns centres, double binned in the horizontal direction (adjusted parallel to the dispersion direction) to provide 760 event centered pixels of 15 microns. Dark count rates were negligible at $\emptyset . \emptyset 65 \mathrm{~Hz} /$ pixel, for both $\mathrm{l}-\mathrm{D}$ and 2-D systems.

The bulk of the Ca II K data was acquired at the $\mathrm{f} / 1 \emptyset$ cassegrain focus of the Siding Spring Observatory Im ( 49 inches) telescope, with a similar echelle grating and 2-D photon counting device. The spectrograph incorporated a $1 \mathrm{~m}$ focal length collimator and $305 \mathrm{~mm}$ camera configuration which yielded a dispersion of 3.9 $\AA / \mathrm{mm}$ at $3933 \AA$ ( 57 th order) and a $44 \AA$ spectral coverage. The overlapping orders were separated by a $6 \emptyset \emptyset$ grooves $/ \mathrm{mm}, 13^{\circ}$ blaze cross-disperser.

Entrance slit widths were typically $20 \emptyset$ microns $(\emptyset .7$ arc seconds on the sky) and $1 \emptyset \emptyset$ microns ( 1 arc second) for the $1.88 \mathrm{~m}$ and $1 \mathrm{~m}$ observations, respectively. These widths were often adjusted according to the quality of the night transparency and seeing (usually ranging 2 to 6 arc seconds), but were never set narrower than to provide a projected slit width of 2 pixels at the detector. Maximum count rates were maintained at below $0.6 \mathrm{~Hz} /$ pixel to ensure linearity of signal response at the detector. 
In Table 2.1 we list the program stars (assembled according to luminosity class), telescopes utilised for their observation, exposure times and observation dates; from which the time interval between $\mathrm{Ca}$ II $\mathrm{K}$ and $\mathrm{H}-\mathrm{alpha}$ observations for a particular star can be inferred. H-alpha and Ca II K observations tabulated for the 1979 period were kindly obtained by Alex Rodgers and Paul Harding, using the 1-D PCA at the $1.88 \mathrm{~m}$ telescope. (Their Ca II K data was observed at $2.4 \mathrm{~A} / \mathrm{mm}$ dispersion). During the course of a night's observations, each stellar spectrum was bracketted by a $50 \emptyset$ seconds exposure of an emission line arc. Neon and iron-argon gas tubes were utilised to provide reference lines in the wavelength regions of $\mathrm{H}-\mathrm{alpha}$ and $\mathrm{Ca}$ II $\mathrm{K}$, respectively. At the end of each night, a 5000 seconds exposure of a diffuse quartz halogen lamp spectrum was obtained for subsequent flat-fielding of the spectra. Finally, the data were written onto magnetic tape and transferred to a Vax $11 / 78 \emptyset$ computer for processing.

\subsection{REDUCTIONS}

\section{(a) Background Subtraction And Flat-fielding}

The sky background in the 1-D PCA spectra was removed by channel per channel subtraction of the sky array from the star counts. Most of the spectral curvature induced by image tube vignetting and echelle blaze effects was eliminated by division of the stellar spectrum with the normalised flatfield exposure 
TABLE 2.1

JOURNAL OF OBSERVATIONS

\begin{tabular}{|c|c|c|c|c|c|c|c|c|c|c|c|c|c|}
\hline \multirow[b]{2}{*}{ Name } & \multicolumn{2}{|r|}{$\mathrm{H} \alpha$} & \multirow[b]{2}{*}{ Date } & \multicolumn{2}{|c|}{ CaIIK } & \multirow[b]{2}{*}{ Date } & \multirow[b]{2}{*}{ Name } & \multicolumn{2}{|c|}{ Ha } & \multirow[b]{2}{*}{ Date } & \multicolumn{2}{|r|}{ CaIIK } & \multirow[b]{2}{*}{ Date } \\
\hline & To1 & $\begin{array}{l}\text { Exp } \\
\text { (s) }\end{array}$ & & Tel & $\begin{array}{l}\text { Exp } \\
(\mathrm{s}) \\
\end{array}$ & & & Tel 1 & $\begin{array}{l}\text { Exp } \\
(\mathrm{s})\end{array}$ & & Te1 & $\begin{array}{l}\text { Exp } \\
(\mathrm{s}) \\
\end{array}$ & \\
\hline \multicolumn{7}{|c|}{ DWARFS } & . & \multicolumn{6}{|c|}{ GIANTS (cont'd) } \\
\hline Moon & 74 & 1000 & $5 / 4 / 82$ & 74 & 2000 & $5 / 4 / 82$ & 51 Hya & 74 & 1500 & $18 / 3 / 81$ & 40 & 1500 & $12 / 5 / 81$ \\
\hline$a$ Cen $A$ & 74 & 600 & $7 / 4 / 81$ & 40 & 1000 & $11 / 5 / 81$ & $\delta$ oph & 74 & 1000 & $19 / 5 / 81$ & 40 & 1500 & $13 / 5 / 81$ \\
\hline B Hyi & 74 & 1000 & $1 / 3 / 79$ & 74 & 2600 & $1 / 3 / 79$ & $\checkmark$ vir & 74 & 900 & $1 / 3 / 79$ & 74 & 3600 & $1 / 3 / 79$ \\
\hline HD 147513 & 74 & 1500 & $19 / 3 / 81$ & 40 & 1500 & $12 / 5 / 81$ & HD 146003 & 74 & 1500 & $19 / 3 / 81$ & 40 & 2000 & $12 / 5 / 81$ \\
\hline K Cet & 74 & 2000 & $17 / 12 / 81$ & 40 & 2000 & $13 / 2 / 82$ & $\psi$ Leo & 74 & 2000 & $8 / 4 / 81$ & 40 & 1500 & $13 / 5 / 81$ \\
\hline ह Во० A & 74 & 2000 & $19 / 5 / 81$ & 40 & 1500 & $11 / 5 / 81$ & $\delta$ vir & 74 & 2000 & $19 / 5 / 81$ & 40 & 1500 & $13 / 5 / 81$ \\
\hline $6 \mathrm{Pav}$ & 74 & 600 & $8 / 4 / 81$ & 40 & 1000 & $11 / 5 / 81$ & $\psi$ vir & 74 & 2000 & $8 / 4 / 81$ & 40 & 2000 & $11 / 5 / 81$ \\
\hline Y, Aps & 74 & 1000 & $8 / 4 / 81$ & 40 & 1000 & $11 / 5 / 81$ & $\psi$ Phe & 74 & 1500 & $17 / 12 / 81$ & 40 & 800 & $11 / 2 / 82$ \\
\hline $0^{2} \mathrm{Er} 1$ & 74 & 1800 & $18 / 3 / 81$ & 40 & 1000 & $23 / 3 / 81$ & $\sigma$ Lib & 74 & 500 & $19 / 3 / 81$ & 40 & 2000 & $24 / 3 / 81$ \\
\hline 70 oph A & 74 & 1500 & $18 / 3 / 81$ & 40 & 1000 & $11 / 5 / 81$ & $\varepsilon$ Mus & 74 & 600 & $7 / 4 / 81$ & 40 & 2000 & $11 / 5 / 81$ \\
\hline 83 Leo & 74 & 2000 & $19 / 3 / \theta 1$ & 40 & 1000 & $12 / 5 / 81$ & $Y$ Ret & 74 & 1000 & $16 / 12 / 81$ & 40 & 2000 & $15 / 2 / 82$ \\
\hline$a$ Cen $B$ & 74 & 800 & $8 / 4 / 81$ & 40 & 1000 & $11 / 5 / 81$ & Y Cru & 74 & 2000 & $10 / 3 / 81$ & 40 & 2000 & $13 / 2 / 81$ \\
\hline$\varepsilon$ Er1 & 74 & 1000 & $15 / 12 / 81$ & 40 & 2500 & $13 / 2 / 82$ & & \multirow{3}{*}{\multicolumn{6}{|c|}{ SUPER GIANTS }} \\
\hline$c$ Ind & 74 & 1000 & $8 / 4 / 81$ & 40 & 1000 & $11 / 5 / 81$ & & & & & & & \\
\hline HD 131977 & 74 & 2000 & $18 / 3 / 81$ & 40 & 2000 & $11 / 5 / 81$ & & & & & & & \\
\hline \multirow{2}{*}{\multicolumn{7}{|c|}{ GIANTS }} & o. Pup & 74 & 1000 & $16 / 12 / 81$ & 40 & 2500 & $15 / 2 / 82$ \\
\hline & & & & & & & $\delta \mathrm{cma}$ & 74 & 800 & $19 / 3 / 81$ & 40 & 1000 & $24 / 3 / 81$ \\
\hline $8 \mathrm{Col}$ & 74 & 900 & $1 / 3 / 79$ & 74 & 2600 & $1 / 3 / 79$ & $\delta$ vol & 74 & 2000 & $22 / 12 / 80$ & 40 & 2000 & $25 / 2 / 81$ \\
\hline B Lep & 74 & 1000 & $1 / 3 / 79$ & 74 & 2400 & $1 / 3 / 79$ & 11 Pup & 74 & 1000 & $11 / 3 / 82$ & 40 & 1000 & $5 / 4 / 82$ \\
\hline u vel & 74 & 1000 & $19 / 5 / 81$ & 40 & 1000 & $11 / 5 / \theta 1$ & HD 57146 & 74 & 1500 & $24 / 12 / 80$ & 40 & 1500 & $12 / 5 / 81$ \\
\hline B Crv & 74 & 1000 & $19 / 5 / 81$ & 40 & 1000 & $13 / 5 / 81$ & HD 59890 & 74 & 1500 & $24 / 12 / 80$ & 40 & 1000 & $12 / 5 / 81$ \\
\hline E. Hya & 74 & 1000 & $17 / 12 / 81$ & 40 & $2000^{\prime}$ & $11 / 2 / 82$ & 5 Mon & 74 & 1500 & $16 / 12 / 81$ & 40 & 2000 & $13 / 2 / 82$ \\
\hline $\mathrm{v}^{1}{ }^{1} \mathrm{Hya}$ & 74 & 2000 & $10 / 3 / 82$ & 40 & 1500 & $15 / 2 / 82$ & 20 Pup & 74 & 1500 & $16 / 12 / 81$ & 40 & 1500 & $15 / 2 / 82$ \\
\hline 5. Lup & 74 & 1500 & $18 / 3 / 81$ & 40 & 1500 & $23 / 3 / 81$ & $\alpha \operatorname{Ret}$ & 74 & 2000 & $15 / 12 / 81$ & 40 & 1200 & $15 / 2 / 82$ \\
\hline$\delta$ Lep & 74 & 1000 & $2 / 3 / 79$ & 74 & 3000 & $2 / 3 / 79$ & $\varepsilon \mathrm{cmi}$ & 74 & 2000 & $19 / 3 / 81$ & 40 & 2000 & $25 / 3 / 81$ \\
\hline$\delta \mathrm{crt}$ & 74 & 1500 & $7 / 4 / 81$ & 40 & 1000 & $24 / 3 / 81$ & HD 165438 & 74 & 2000 & $19 / 5 / 81$ & 40 & 2000 & $12 / 5 / 81$ \\
\hline I Phe & 74 & 1000 & $2 / 2 / 82$ & 40 & 2000 & $15 / 2 / 82$ & IID 93070 & 74 & 2000 & $18 / 3 / 81$ & 40 & 1500 & $13 / 5 / \theta 1$ \\
\hline c vir & 74 & 2000 & $19 / 5 / 81$ & 40 & 1000 & 13/5/81 & HD 65662 & 74 & 1000 & $24 / 12 / 80$ & 40 & 2000 & $13 / 5 / 81$ \\
\hline a Phe : & 74 & $1000=$ & $2 / 3 / 79$ & 74 & 2400 & $2 / 3 / 79$ & HD 152781 & 74 & 2000 & $19 / 3 / 81$ & 40 & 2000 & $12 / 5 / 81$ \\
\hline T Pup & 74 & $1000^{\circ}$ & $19 / 3 / 81$ & 40 & 2000 & $23 / 3 / 61$ & $v^{1} \operatorname{sgr}$ & 74 & 1000 & $8 / 4 / 81$ & 40 & 1000 & $11 / 5 / 81$ \\
\hline$v^{2} E r i$ & 74 & 1000 & $18 / 3 / 81$ & 40 & 2000 & $23 / 3 / 81$ & ID 68553 & 74 & 1000 & $24 / 12 / 80$ & 40 & 1500 & $12 / 5 / 81$ \\
\hline HD 94510 & 74 & 1000 & $7 / 4 / 81$ & 40 & 1500 & $11 / 5 / 81$ & HD 91942 & 74 & 1000 & $7 / 4 / 81$ & 40 & 1500 & $12 / 5 / 81$ \\
\hline e $\mathrm{Col}$ & 74 & 1000 & $6 / 4 / 79$ & 74 & 2500 & $6 / 4 / 79$ & B Ara & 74 & 1200 & $18 / 3 / 81$ & 4J & 1000 & $11 / 5 / 81$ \\
\hline B Cet & 74 & 1000 & $5 / 4 / 79$ & 74 & 2500 & $5 / 4 / 79$ & a Tra & 74 & 800 & $8 / 4 / 81$ & 40 & 1000 & $11 / 5 / 81$ \\
\hline a Boo & 74 & 600 & $8 / 4 / 81$ & 40 & 1000 & $11 / 5 / 81$ & Y Phe & 74 & 1000 & 19/3/81 & 40 & 1000 & $24 / 3 / 81$ \\
\hline$\pi$ Hys & 74 & 1000 & $7 / 4 / 81$ & 40 & 1000 & $12 / 5 / 81$ & $\pi$ Pup & 74 & 1000 & $8 / 4 / 81$ & 40 & 2000 & $24 / 3 / 81$ \\
\hline e sco & 74 & 600 & $8 / 4 / 81$ & 40 & 1500 & $24 / 3 / 81$ & HD 89388 & 74 & 1200 & $18 / 3 / 81$ & 40 & 1500 & $12 / 5 / 81$ \\
\hline HO 101067 & 74 & 2000 & $8 / 4 / 81$ & 40 & 1500 & $11 / 5 / 81$ & $\lambda$ Vel & 74 & 720 & $8 / 4 / 81$ & 40 & 1200 & $11 / 5 / 81$ \\
\hline $8 \mathrm{col}$ & 74 & 2000 & $18 / 3 / 81$ & 40 & 1200 & $13 / 5 / 81$ & IID 63032 & 74 & 1000 & $7 / 4 / 81$ & 40 & 1500 & $12 / 3 / 81$ \\
\hline$\checkmark$ Hya & 74 & 1000 & $7 / 4 / 81$ & 40 & 3000 & $23 / 3 / 81$ & a cma & 74 & 1200 & $22 / 12 / 80$ & 40 & 1300 & $24 / 3 / 81$ \\
\hline a $\mathrm{T} \cdot \mathrm{jc}$ & 74 & 600 & $8 / 4 / 81$ & 40 & 1000 & $12 / 5 / 81$ & HD 66342 & 74 & 2000 & $13 / 3 / 81$ & 40 & 2000 & $13 / 5 / 81$ \\
\hline $\mathrm{B} \mathrm{Cnc}$ & 74 & 2000 & $22 / 12 / 80$ & 40 & 1000 & $13 / 5 / 81$ & 11091056 & 74 & 2000 & $18 / 3 / 81$ & 40 & 2000 & $13 / 5 / 81$ \\
\hline o Pup & 74 & 1000 & $19 / 3 / 81$ & 40 & 1000 & $26 / 2 / 01$ & a Sco & 74 & 000 & $7 / 4 / 81$ & 40 & 1000 & $11 / 5 / 81$ \\
\hline N vel & 74 & 1000 & $\theta / 4 / \theta 1$ & 40 & 1500 & $12 / 5 / 81$ & a or 1 & 74 & 1000 & $11 / 3 / 82$ & 40 & 2000 & $15 / 2 / 82$ \\
\hline c Lep & 74 & 1000 & $6 / 4 / 79$ & 74 & 2400 & $6 / 4 / 79$ & $a^{\prime}$ ller & 74 & 1000 & $19 / 5 / 81$ & 40 & 1000 & $13 / 5 / 81$ \\
\hline
\end{tabular}


corresponding to the nights observations. The latter procedure also corrected for pixel to pixel sensitivity variations and coherent clock noise levels superimposed on the array output signals.

For the 2-D PCA observations, the spectral orders were generally spread over 5-6 rows of the CCD array, (depending upon seeing conditions). A mean sky background was estimated by taking the average over 6 rows of counts in the inter-order regions on both sides of the order. Pixel by pixel division of [star minus background by \{corresponding flat-field row was then performed separately for the rows containing the spectrum.

\section{(b) Wavelength Calibration}

Wavelength scales for the Ca II $K$ profiles were derived from the arc exposures by averaging the "before" and "after" lamp spectra (thus accounting for possible instrumental drifts) and computing the pixel positions corresponding to the centroids of several arc emission lines of known laboratory wavelengths. Table 2.2 lists the Fe-Ar lines identified for this purpose. Arc centroid positions were generally computed to a precision of 1 pixel (about $\emptyset . \emptyset 6 \AA$ ) and a wavelength-pixel relation was determined by a least squares calculation performed to this tolerance. Each calibrated spectrum was then aligned with respect to photospheric absorption lines of known wavelength (e.g. FeI at $3930.308 \AA$ ) so as to place the Ca II $K$ feature in the rest frame of the stellar 
TABLE 2.2

IRON - ARGON COMPARISON LINES IDENTIFIED FOR CAII K WAVELENGTH CALIBRATION

\begin{tabular}{lc}
\hline Element & ${ }^{*}$ Laboratory wavelength [A] \\
\hline Ar I & 3920.26 \\
Ar I & 3922.91 \\
Ar I & 3925.95 \\
Ar I & 3927.92 \\
Ar I & 3930.30 \\
Ar I & 3942.44 \\
Fe I & 3947.51 \\
Fe I & 3948.98 \\
\hline
\end{tabular}

* From Gray (1972) 
photosphere.

In most cases, the neon arc exposures contained insufficient comparison lines for an accurate wavelength calibration of the H-alpha data. Alternatively, we used unblended photospheric absorption lines in the neighbourhood of H-alpha to automatically establish a wavelength scale relative to the stellar photosphere. The absorption line centroids were calculated to a precision of 2 pixels $(\sim \varnothing .1 \AA)$ and the associated wavelengths (Table 2.3) were determined by reference to the Arcturus (Griffin, 1968) and solar (Minnaert et al., 1940) spectral atlases.

For the 2-D PCA data, a first order polynomial fit was performed for each row of the arc spectrum and the resulting calibrations applied to the corresponding rows of the stellar spectral order. Each row was then shifted to coincide in wavelength and summed to produce a composite spectrum of high signal to noise. Comparison lines obtained with the 1-D system required a third order polynomial fit to correct for the non-linear wavelength-pixel mapping caused by "pin cushion" distortions of the tandem electrostatic tubes. In order to facilitate comparisons of $\mathrm{H}-a l$ pha and $\mathrm{Ca}$ II $K$ profiles, the respective wavelength scales were converted into velocity units through the equation,

$$
V=c\left(\lambda-\lambda_{0}\right) / \lambda_{0}
$$

where $\lambda_{0}$ is the line rest wavelength, (6562.808 $\AA$ and 3933.682 $\AA$ for H-alpha and Ca II K, respectively). 
TABLE 2.3

PHOTOSPHERIC ABSORPTION LINES IDENTIFIED FOR $\mathrm{H} \alpha$ WAVELENGTH CALIBRATION

Element

* Solar spectrum wavelength [\&]

$\mathrm{Fe} \mathrm{I}$

Ti I

Si I

Ti I

$\mathrm{Ti}$ I I

Fe I

Ca I

$\mathrm{Fe} \mathrm{I}$

$\mathrm{Fe} I$
6546.25

6554.24

6555.47

6556.08

6559.58

6569.22

6572.80

6574.25

6575.04

* From Moore et al. (1966) 


\section{(c) Spectral Resolution And Profile Filtering}

We estimate the spectral resolution of the data by assuming a gaussian instrumental profile with a FWHM approximately equal to the square root of the sum of the squares of the limiting resolutions of the main optical components in the spectrograph plus detector system. For the H-alpha profiles observed with the 1-D system, we take 28.8 microns for the mean projected slit width in the spectrograph exit plane and 12.5 microns for the array bin resolution. These widths combine to give an instrumental resolution of 31.4 microns, translating to $0.13 \AA$ at $4 \AA / \mathrm{mm}$. A similar calculation for the Ca II $\mathrm{K}$ data obtained with the 2-D detector, using 31 microns projected slit width and 15 microns pixel resolution, yields 34.4 microns or $\emptyset .13 \AA$ at $3.9 \AA / \mathrm{mm}$.

The above estimates are slightly less than the full widths at half intensities demonstrated by the gas lamp emission lines which were typically $\emptyset .18 \AA$ (about 3 pixels) for both detector systems. We therefore adopt $0.18 \AA$ as the mean spectral resolution of the $\mathrm{H}-\mathrm{alpha}$ and $\mathrm{Ca}$ II K profiles, and equate it to the instrumental broadening of the observational system.

A further degradation in resolution was introduced through spatial filtering of the profiles by convolution with a $\emptyset .1 \AA$ FWHM gaussian function normalised to unit integrated area. This choice of width was optimum in removal of extraneous high frequency noise, whilst retaining low frequency information. Taking into account 
the additional broadening induced by the filtering process, we compute a net spectral resolution of $0.21 \AA$, which corresponds to $16 \mathrm{~km} / \mathrm{s}$ and $9 \mathrm{~km} / \mathrm{s}$ for the Ca II $\mathrm{K}$ and $H-a l p h a$ profiles, respectively.

\section{(d) Definition of Continuum Levels}

Photospheric continuum levels for the H-alpha profiles were determined by reference to line free regions in the previously cited spectral atlases. For each spectrum, we approximated the continuum by a straight line (or a series of straight lines in the case of a poorly flatfielded, sloping spectrum) over a $30 \AA$ bandpass centered at H-alpha. The profiles were then normalised by division with the estimated continuum functions.

Photospheric continua for the Ca II K profiles could not be readily assigned owing to the narrow wavelength coverage of the observations. The amount of usable spectrum, free from edge effects, was limited to approximately $\pm 15 \AA$ of line centre, and only extended to the outer wings of the broad Lorentzian profile of the $\mathrm{Ca}$ II $\mathrm{K}$ line. Alternatively, we normalised each Ca II $\mathrm{K}$ spectrum to a reference level located in the line wing at $3939.682 \AA(\Delta \lambda=6 \AA)$. The latter point is free of significant blanketing by weak lines and is sufficiently far from Ca II $K$ line centre that (a) flux contamination by chromospheric emissions (such as from stellar analogs of 
plages and networks) is small compared to the radiative equilibrium, photospheric component of the wing flux, and (b) it is not likely to be affected by Doppler shifted chromospheric or circumstellar components.

In principle, our arbitrary photometric scale can be calibrated by non-LTE model computations of the Ca II $K$ wing flux at the chosen reference level, as is commonly conducted to produce synthetic spectra for differential abundance analyses. However, this calculation is complicated by uncertainties in upper photospheric temperature and line blanketing distributions, and in assumed values of stellar parameters: effective temperature, surface gravity, metal abundances and calcium line broadening coefficients (Ayres, 1975). Such a calibration for each of the survey stars is beyond the scope of this thesis. Nevertheless, the normalised wing intensity scale offers an approximate means of comparing the relative behaviours of Ca II K profiles, provided such comparisons are confined to stars of similar photospheric properties (e.g. Teff, g, $[\mathrm{Fe} / \mathrm{H}]$ ).

\section{(e) Scattered Light Effects}

Even modern echelles scatter a significant amount of neighbouring continuum light into the core of a deep line. In order to examine the effects of instrumental scattered light, we observed the Moon and Arcturus (K2 III) during this program so that we could compare our observations with the high quality spectra available for these objects. Arcturus was observed in a manner typical 
of our observations of other stars in the spectral survey. The Moon, as an extended source along the spectrograph slit, provided some additional problems in light baffling in the spectrograph, despite the use of appropriate slit masks.

In the upper panel of Figure 2.1a, we plot the 1-D PCA H-alpha profile for Arcturus, together with its counterpart from the Griffin (1968) atlas, on common photospheric velocity and continuum intensity scales. The zero intensity and continuum levels are annotated on the diagram. The Griffin profile was smoothed with a $\emptyset .21 \AA$ gaussian function in order to simulate the spectral resolution of the observations. Comparison of the spectra reveals favorable agreement, in terms of width, and it is clear that the scattered light level in the echelle plus 1-D PCA configuration has a very small effect on the observed H-alpha core intensity.

A similar comparison of solar H-alpha profiles is made in the upper portion of figure $2.1 \mathrm{~b}$, where representative residual intensity points from the Utrecht (1940) Solar atlas (which relates to the centre of the solar disc) are transcribed upon the 2-D PCA lunar exposure. As in the case of Arcturus, the two spectra are congruent in the line wings, but with a slight discrepancy (about $2 \%$ ) in the cores attributable to scattered light.

Since residual intensities of the Ca II $\mathrm{K}$ line are typically 4 to 58 , a small percentage of scattered light will have a more pronounced effect on the core of this 


\section{FIGURE $2.1 \mathrm{a}$}

A comparison of echelle and Griffin (1968) spectra for H-alpha (upper panel) and Ca II K (lower panel) is shown for Arcturus. The Griffin H-alpha spectrum has been convolved with a 0.21 \& gaussian function in order to simulate the instrumental broadening. The $\mathrm{H}$-alpha zero and continuum levels are marked. At Ca II $K$, we overlay the unconvolved Griffin profile upon our observed spectrum. The latter are compared on the normalised intensity scale defined in the text. 


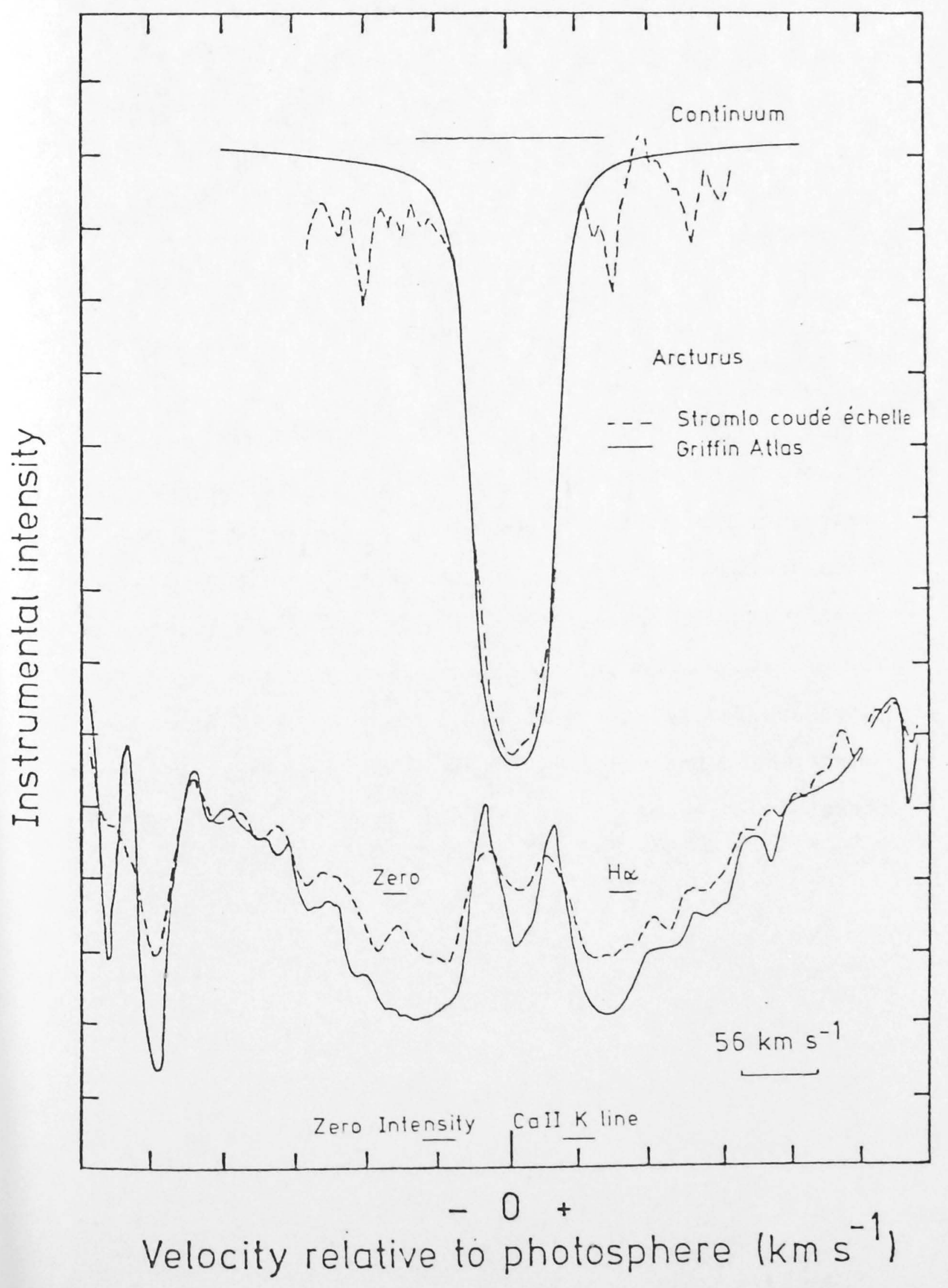




\section{FIGURE $2.1 \mathrm{~b}$}

Similar to Fig. 2.1a, except that comparisons are made between our lunar observations and corresponding profiles from the Utrecht Solar Atlas. Note that the observed H-alpha profile is non-flatfielded. The estimated continuum level is drawn as a smooth arc line. Open circles denote residual intensity points from the solar atlas relative to this continuum. Similarly, open circles at Ca II K represent points from the solar atlas normalised as defined in the text. 
made

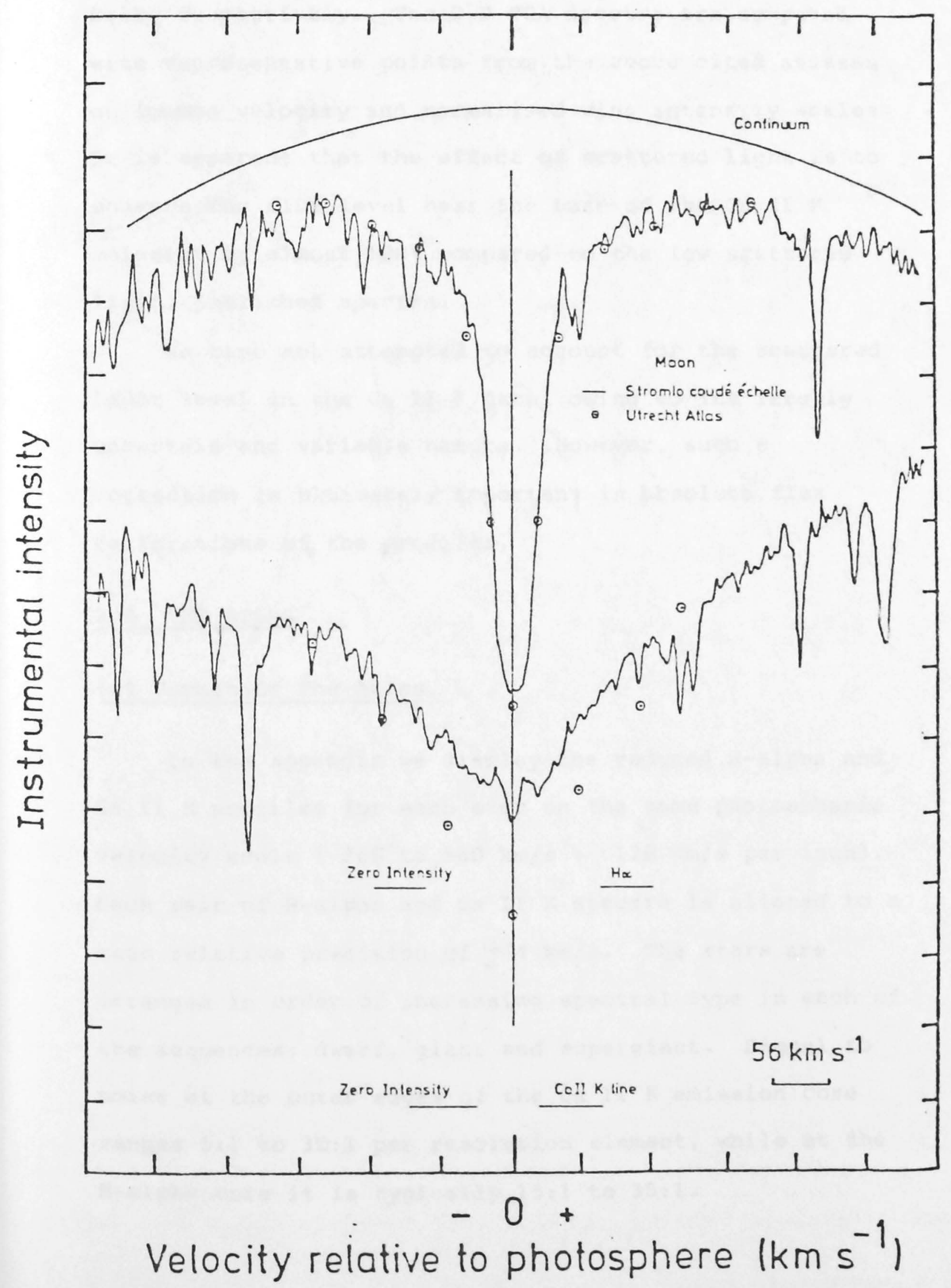


line. The extent of scattered light contamination is graphically illustrated for the Arcturus and lunar Ca II $K$ profiles in the lower sections of figures $2.1 \mathrm{a}$ and $2.1 \mathrm{~b}$, respectively. The 2-D PCA spectra are compared with representative points from the above cited atlases on common velocity and normalised wing intensity scales. It is apparent that the effect of scattered light is to enhance the flux level near the base of the Ca II $K$ emission by almost $100 \%$ compared to the low scattered light, published spectra.

We have not attempted to account for the scattered light level in the Ca II K data, owing to its largely uncertain and variable nature. However, such a correction is ultimately important in absolute flux calibrations of the profiles.

\subsection{THE ATLAS}

\section{(a) Format of The Atlas}

In the appendix we display the reduced $\mathrm{H}$-alpha and Ca II $K$ profiles for each star on the same photospheric velocity scale $(-360$ to $360 \mathrm{~km} / \mathrm{s}$ : $120 \mathrm{~km} / \mathrm{s}$ per inch). Each pair of $\mathrm{H}-\mathrm{al}$ pha and $\mathrm{Ca}$ II $\mathrm{K}$ spectra is aligned to a mean relative precision of $\pm 4 \mathrm{~km} / \mathrm{s}$. The stars are arranged in order of increasing spectral type in each of the sequences: dwarf, giant and supergiant. Signal to noise at the outer edges of the Ca II $\mathrm{K}$ emission core ranges $5: 1$ to $30: 1$ per resolution element, while at the H-alpha core it is typically $15: 1$ to $35: 1$. 
The vertical intensity scale is in units of the normalised $\mathrm{Ca}$ II $\mathrm{K}$ wing counts (as defined in section 2.3). On this scale the zero intensity for the overlayed H-alpha profile is set at ordinate $\emptyset .4$, with the adjudged continuum level corresponding to 1.4. ( $\mathrm{H}$-alpha is plotted on a scale of $\emptyset .2$ residual flux units per inch).

\section{(b) Photospheric LTE H-alpha Profiles}

We have used the ATLAS code (Kurucz, 1970) to compute LTE H-alpha lines from radiative equilibrium model photospheres with effective temperatures in the spectral range spanned by our survey. Figure 2.2 displays the grid of profiles for representative spectral types at $\log (g)=3$ and 4 appropriate to giants and dwarfs, respectively. Solar metal abundances and constant microturbulence of $2 \mathrm{~km} / \mathrm{s}$ were assumed for the calculations. The profiles are plotted on the same intensity and velocity scale as the atlas data, but have been displaced in the vertical direction so as to facilitate direct comparisons.

Our only purpose in deriving these spectra is to empirically illustrate that, while LTE profiles can provide reasonably good fits to the observed wings of the hotter low luminosity stars (e.g. G5 V), there is an "excess" absorption relative to the LTE profile wings in the majority of the cooler Population I giants and supergiants (e.g. K5 III). Presumably, this disparity is due to the influence of a non-LTE chromospheric component in the H-alpha line of late type stars (refer to Chapter One). 


\section{FIGURE 2.2}

H-alpha profiles computed in LTE for flux constant photospheres representative of G0, G5, KO and K5 giants, and $\mathrm{G} 0$ and $K 5$ dwarfs. The profiles are plotted on the same velocity and continuum scale $(0.2$ residual intensity units/inch) as the atlas data, and have been displaced in the vertical direction so as to facilitate comparisons with the observations. Continuum levels are marked. In the text, we call attention to the lack of validity of these computations at 1 ine centre. 
and

in the

the

we

tions

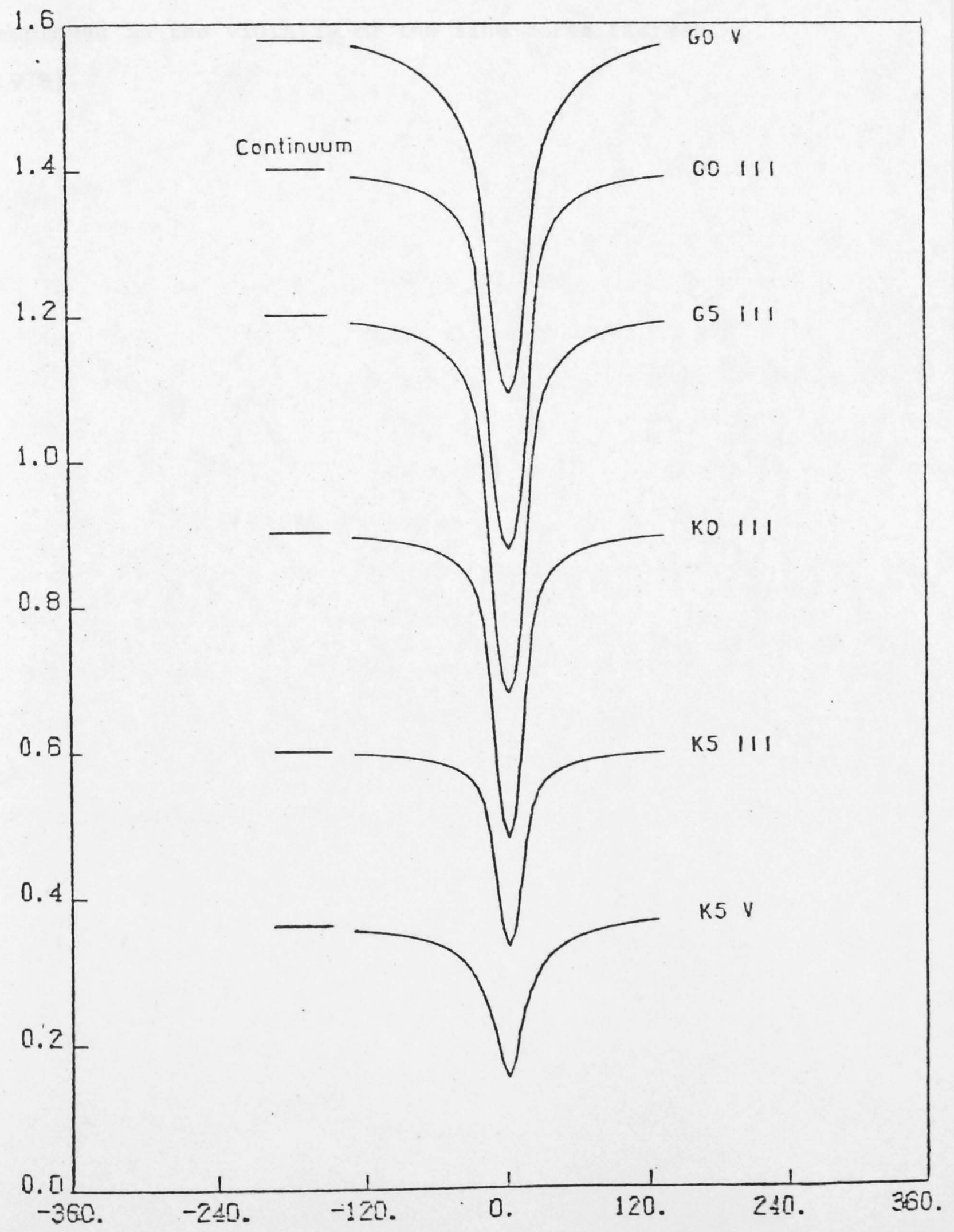

$V[\mathrm{KM} / \mathrm{S}]$ 
Quantitative comparisons between our observed and synthetic line cores are precluded by uncertainties in upper photospheric temperature structure and difficulties associated with $\mathrm{H}-a l$ pha line broadening approximations employed in the vicinity of the line cores (Kurucz, 1979). 


\section{APPENDIX}

An atlas of H-alpha and Ca II $\mathrm{K}$ profiles is presented for the stars in Table 2.1 (excluding the Arcturus and lunar observations which are illustrated in Figs. 2.1a and 2.1b, respectively). Horizontal scale is in units of the photospheric rest velocity. Vertical scale is in units of the normalised $\mathrm{Ca} I I \mathrm{~K}$ intensity defined in text, with zero intensity at 0.0 and unity corresponding to the wing reference level at $3939.682 \AA$. Zero intensity for H-alpha is at ordinate 0.4 , while continum level is at ordinate 1.4 . Stellar identifications and classifications are labelled in the top left hand corner of each panel. Sources of the latter are given in Chapter Three. 


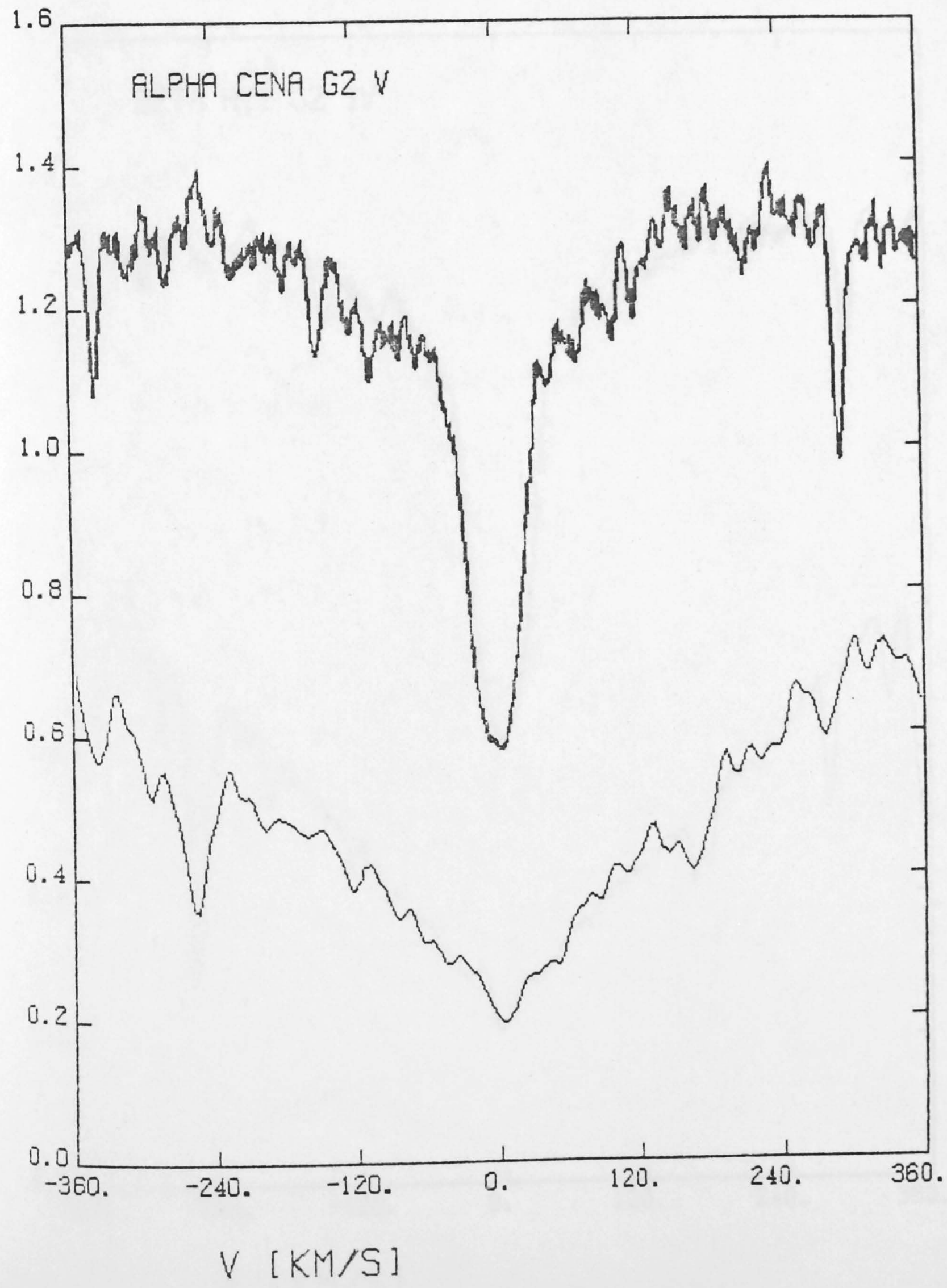




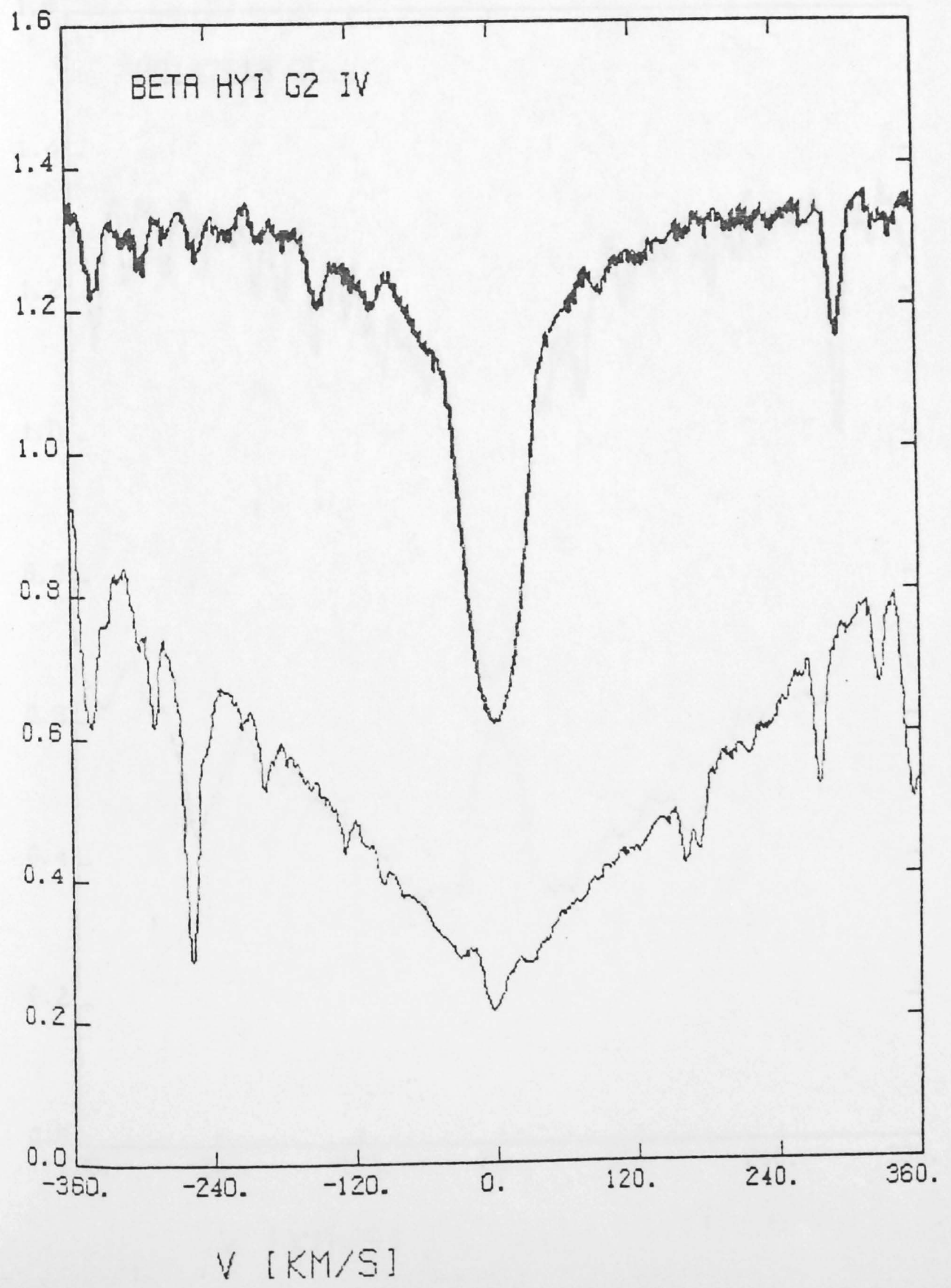




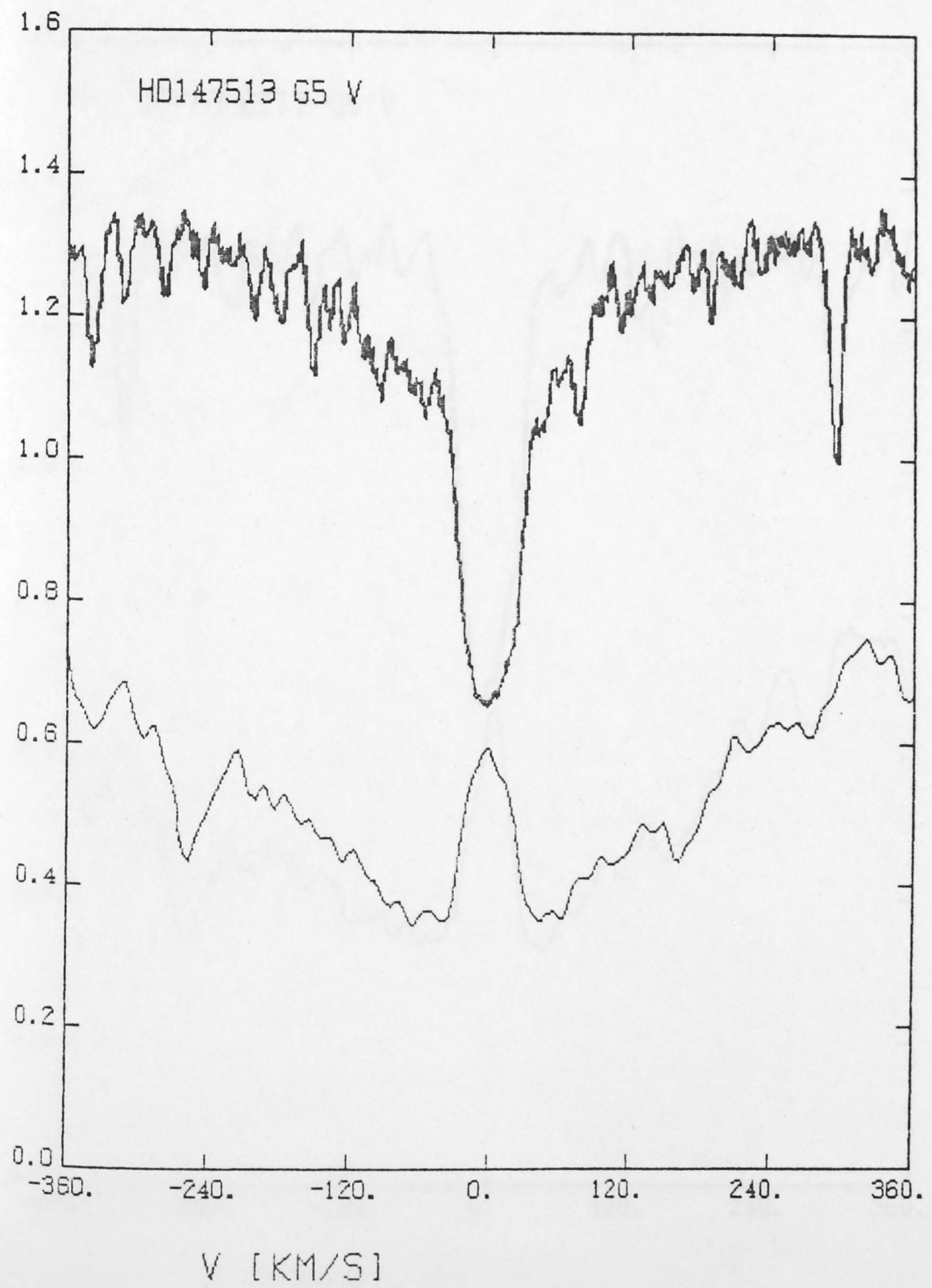




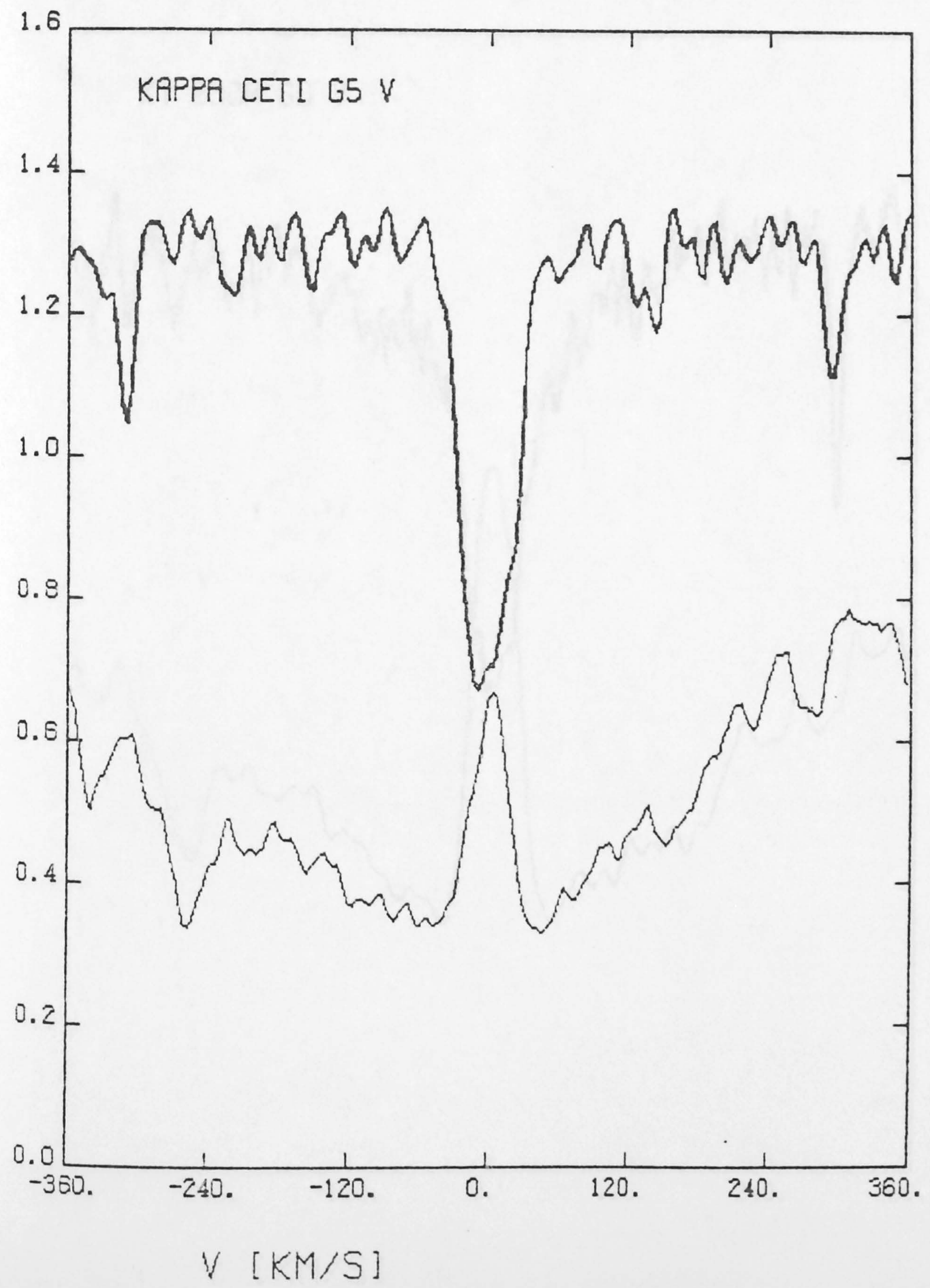




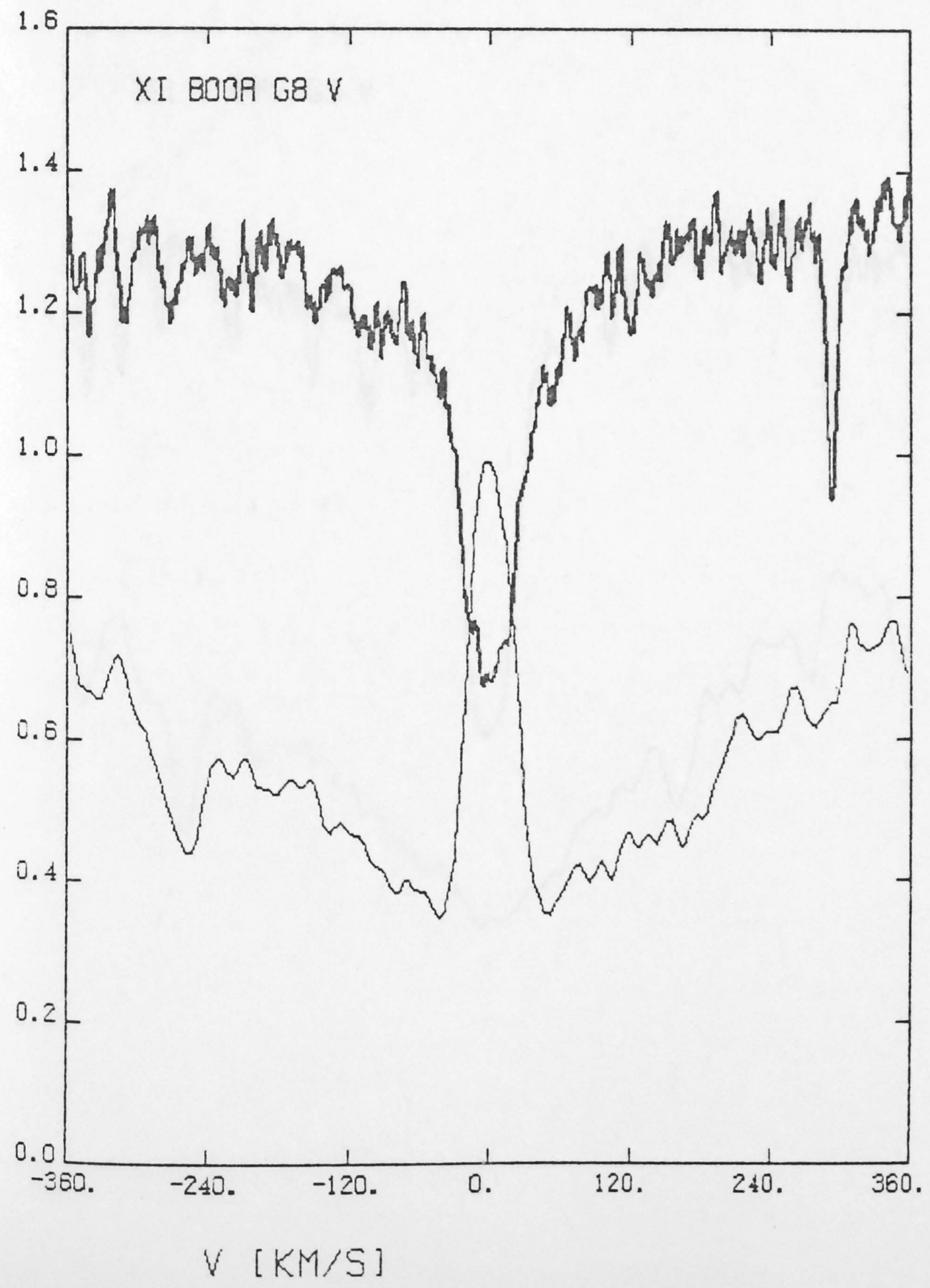




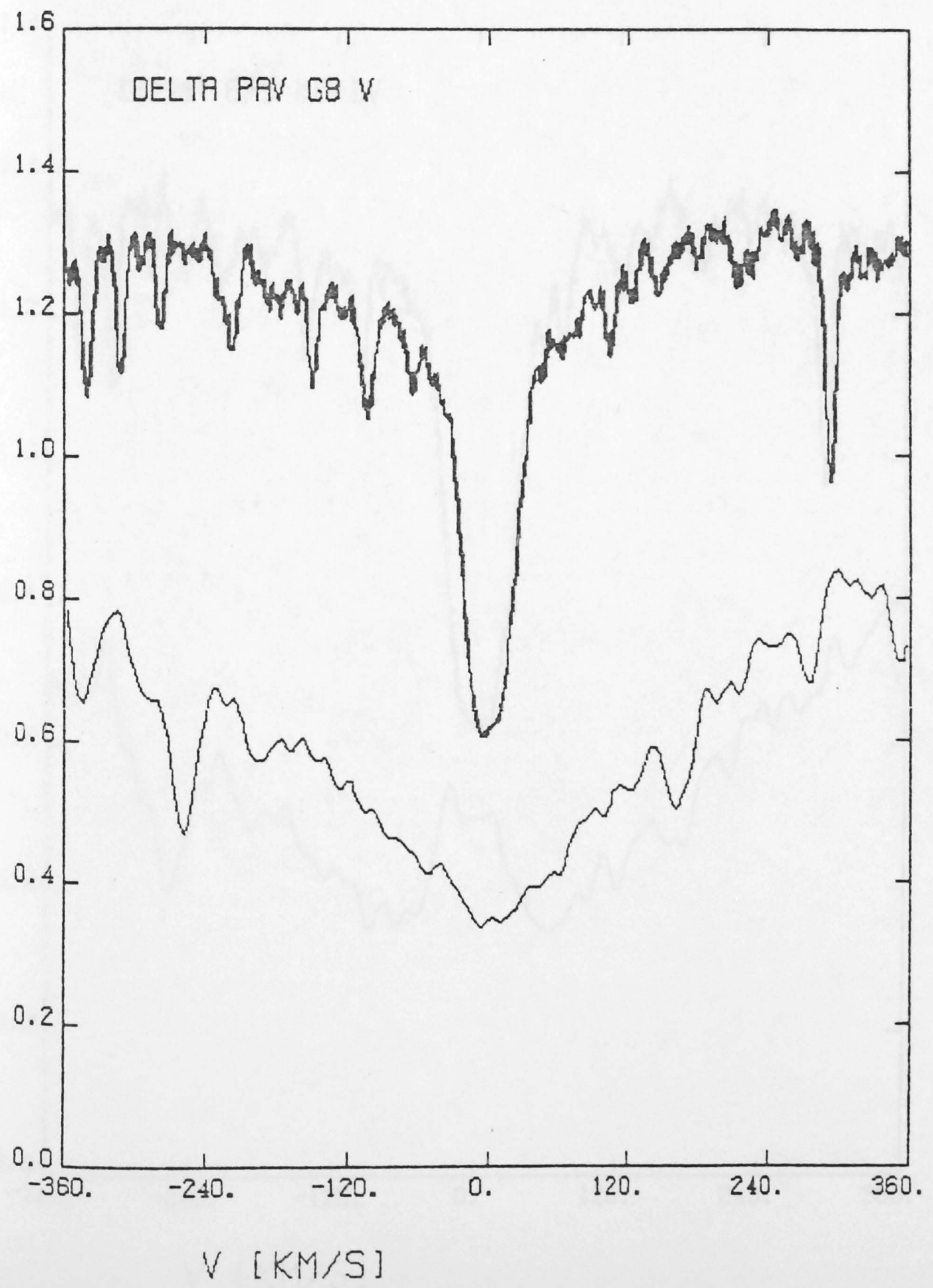




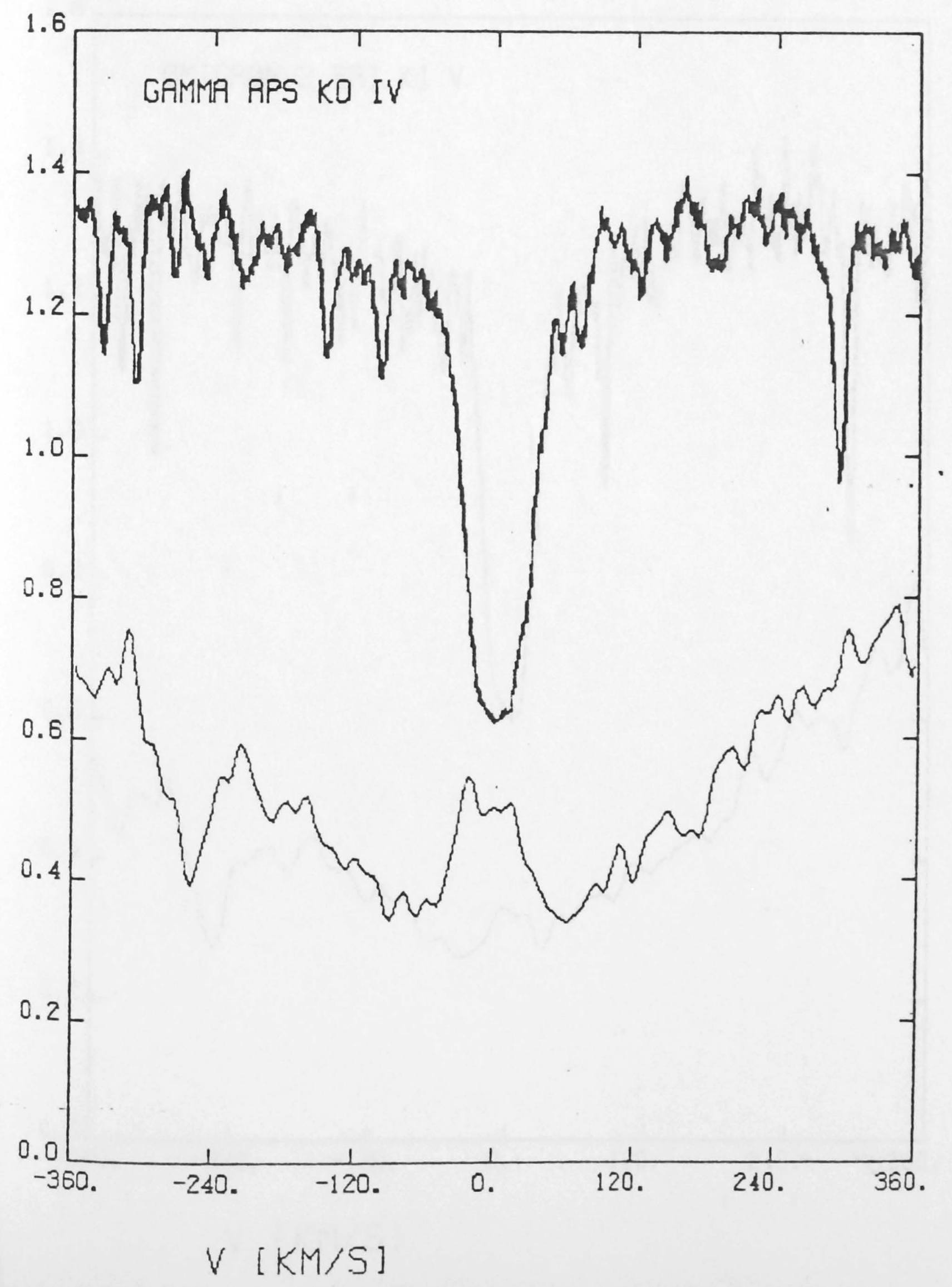




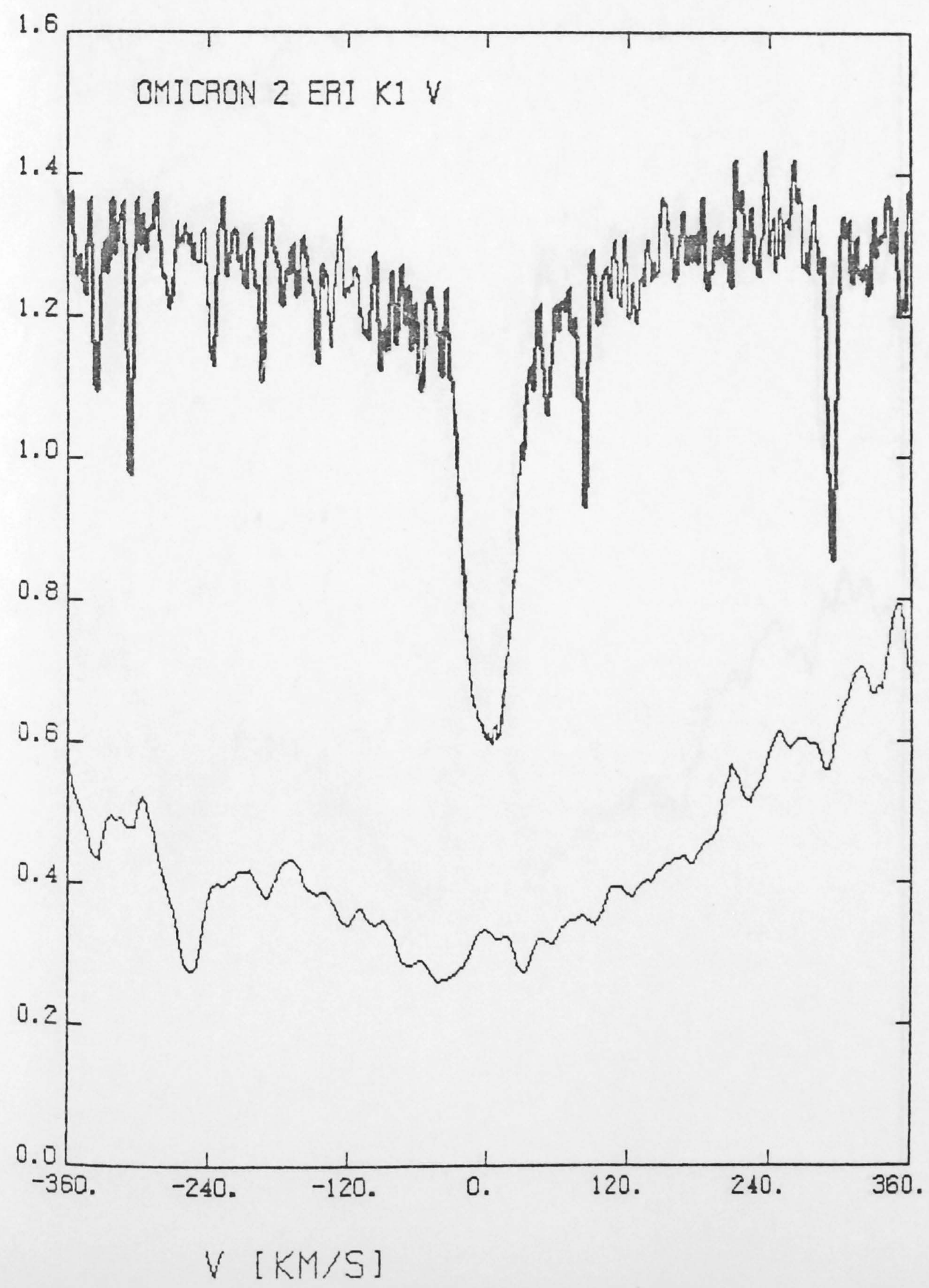




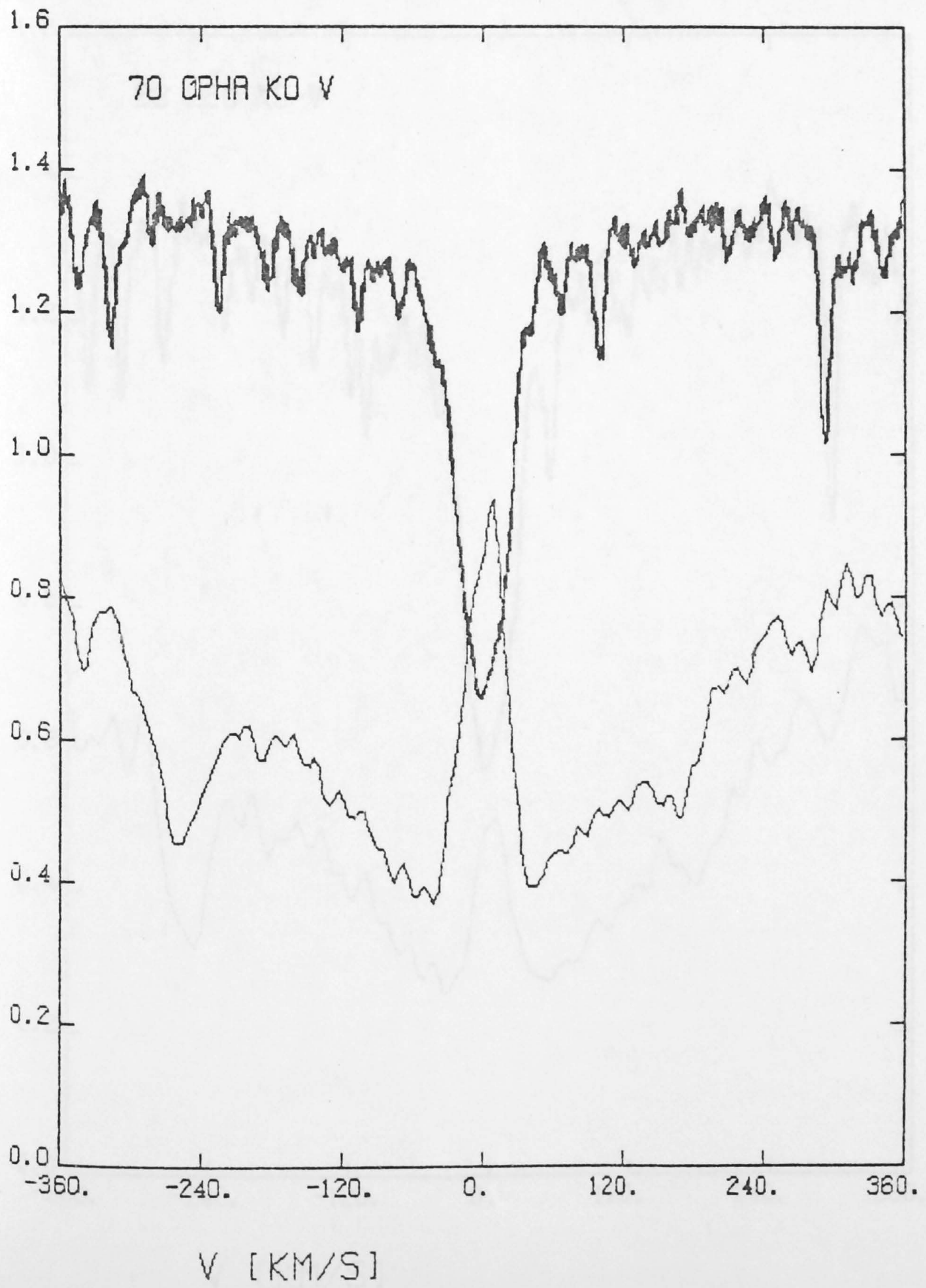




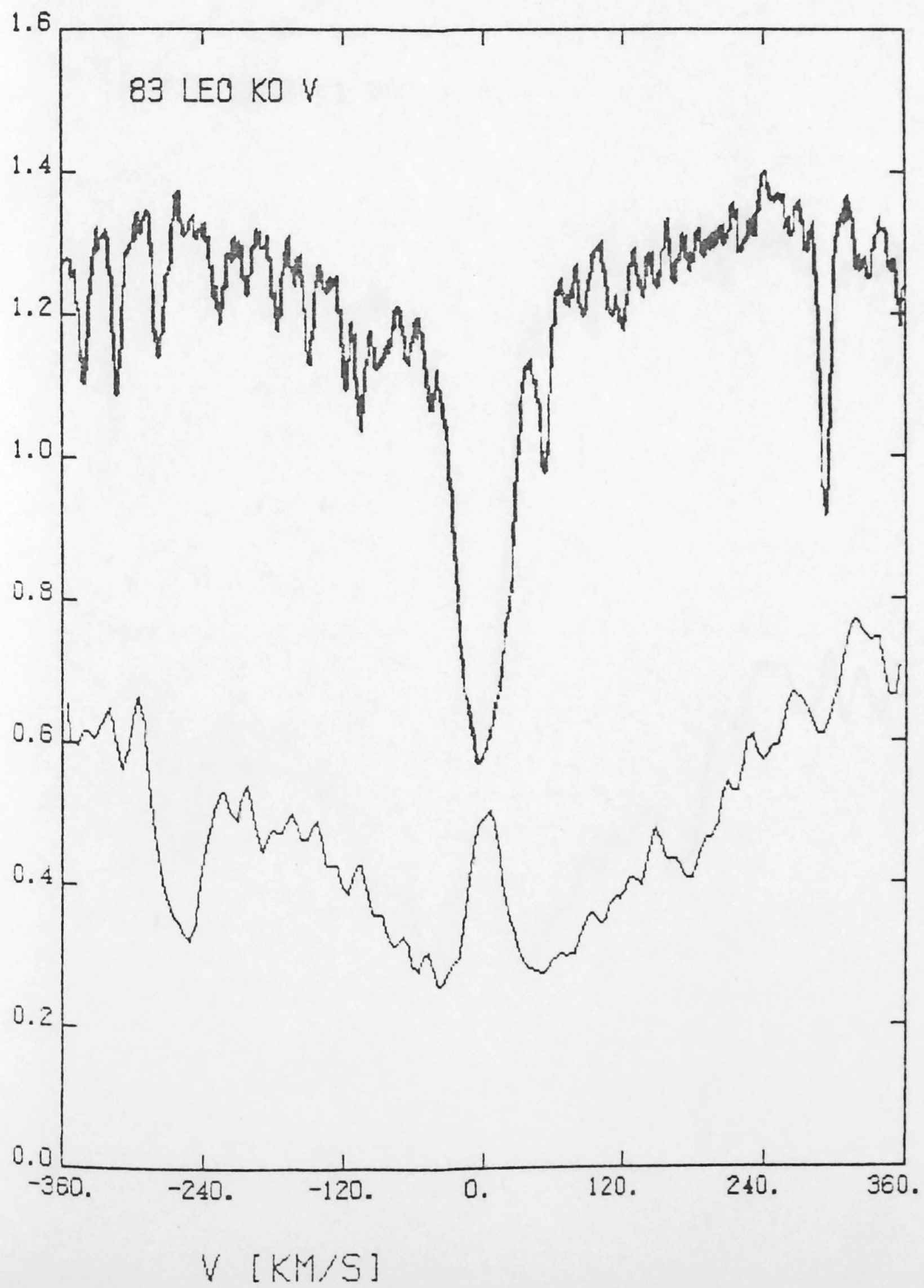




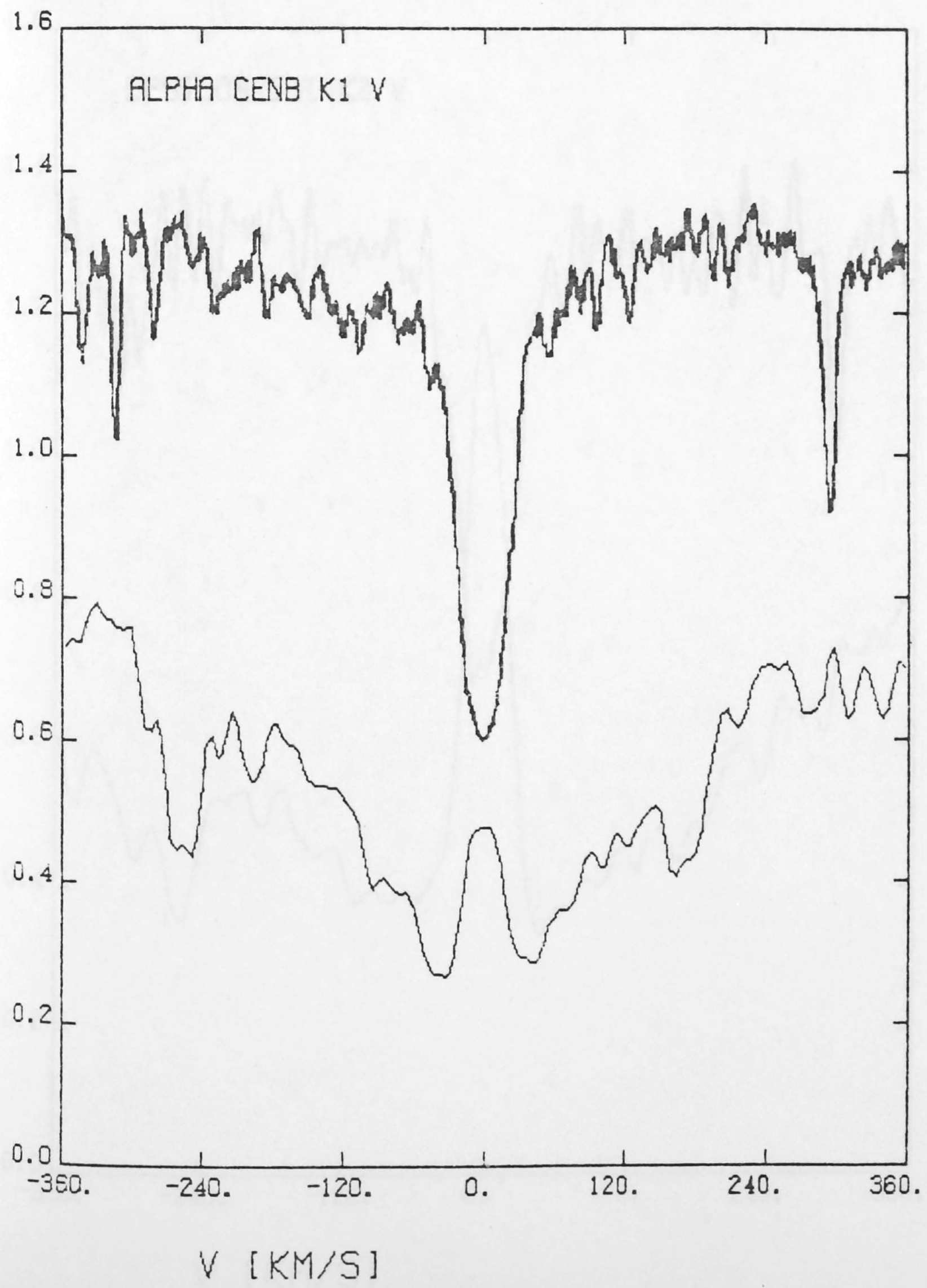




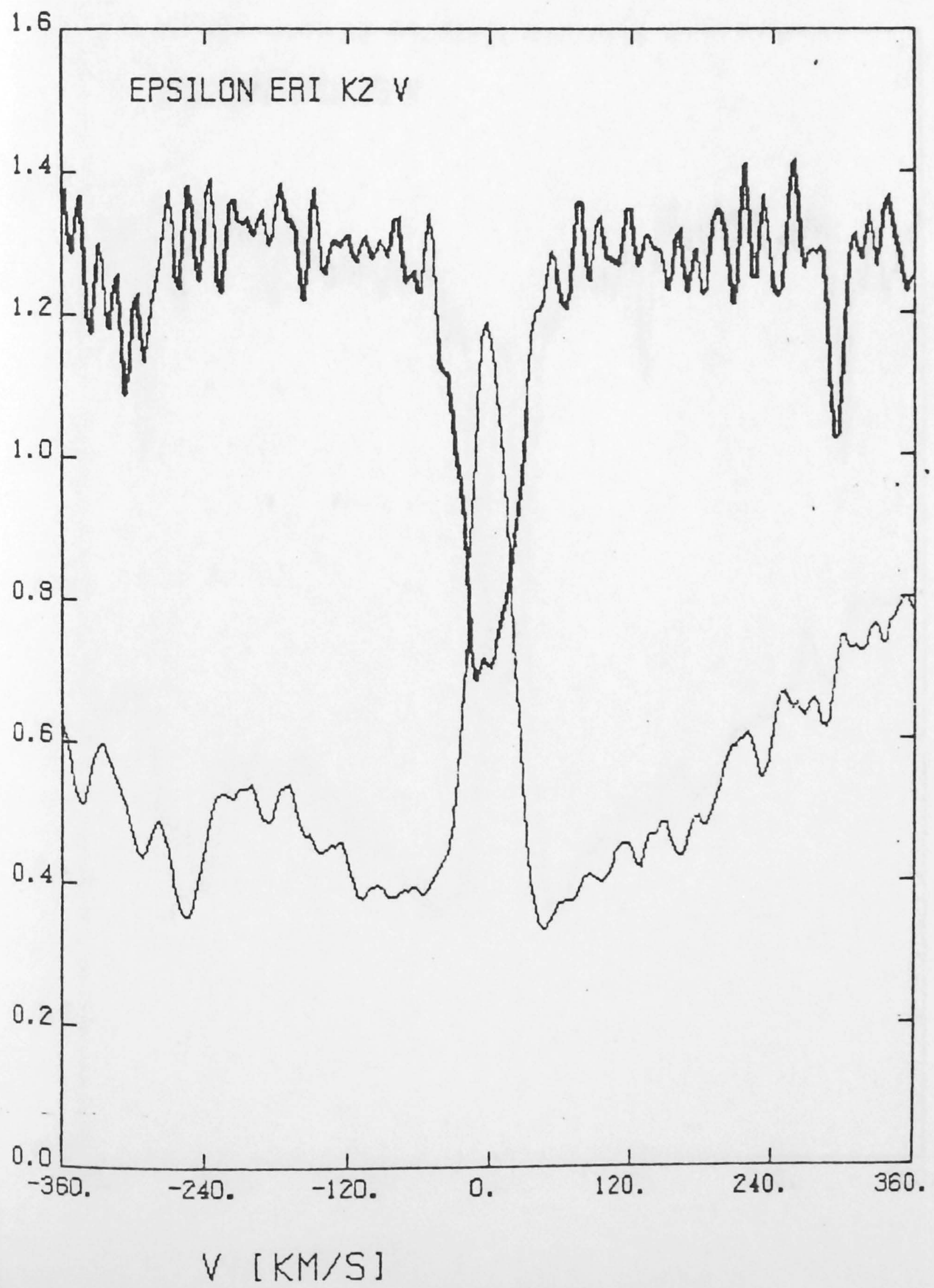




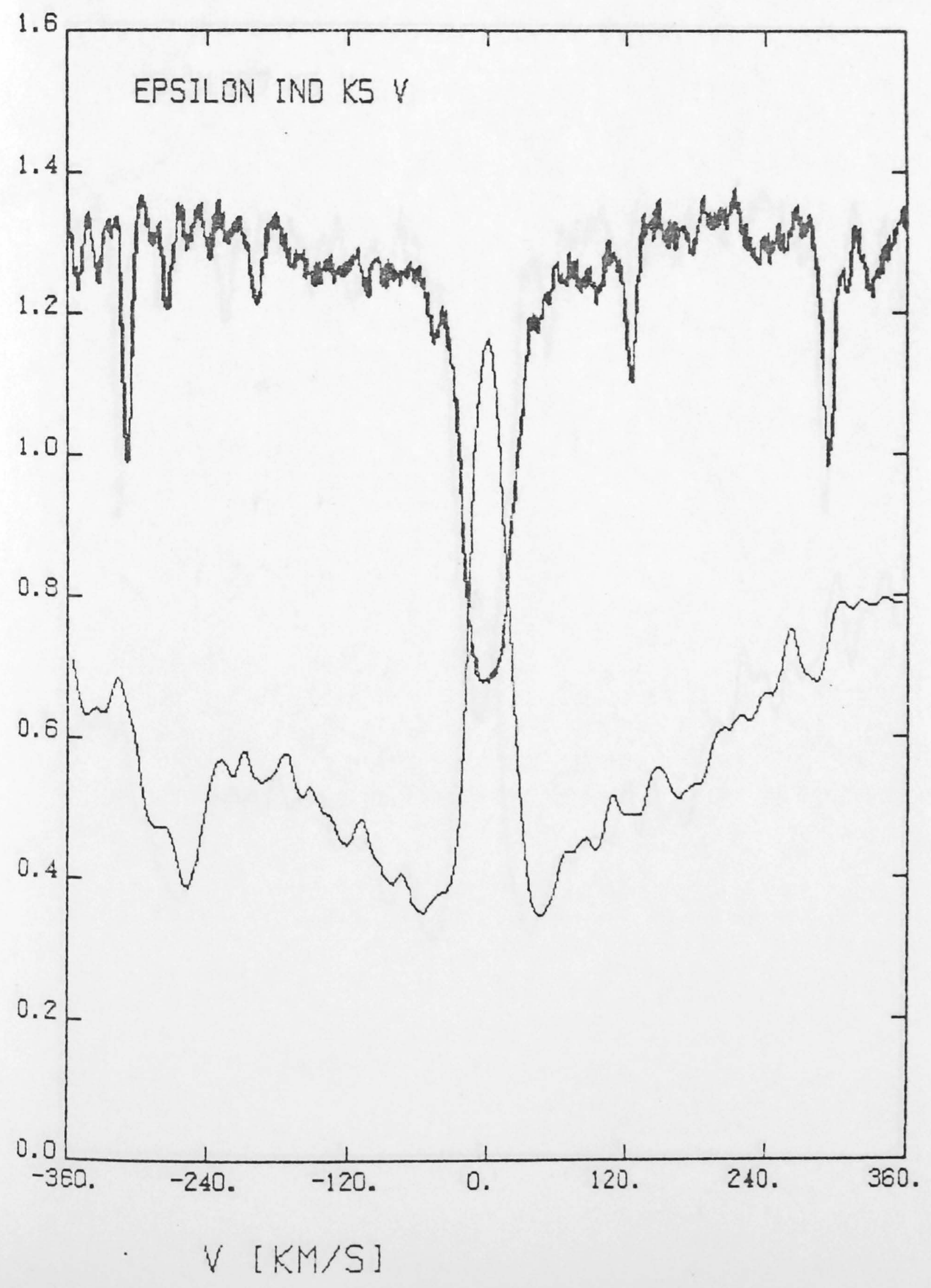




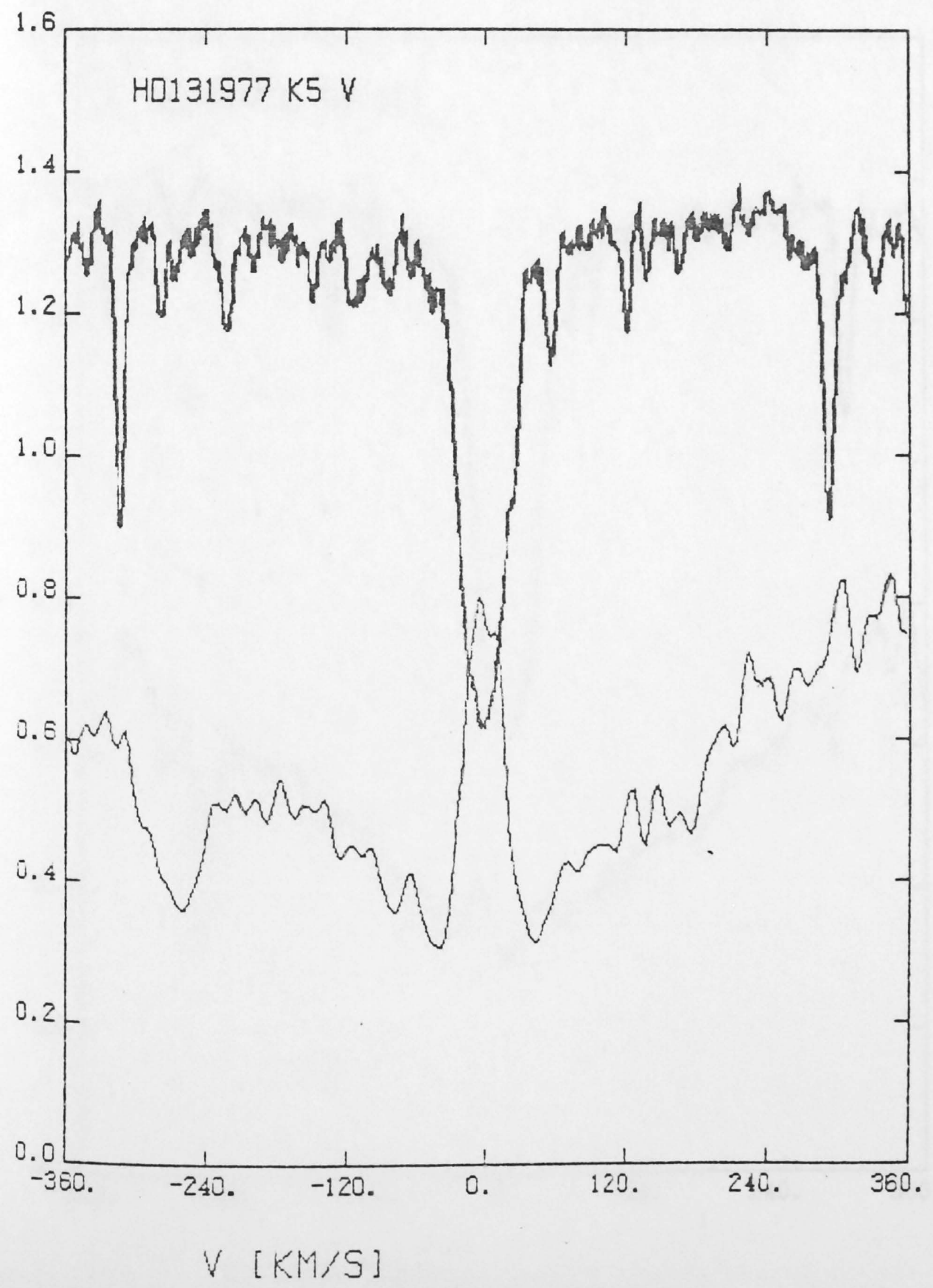




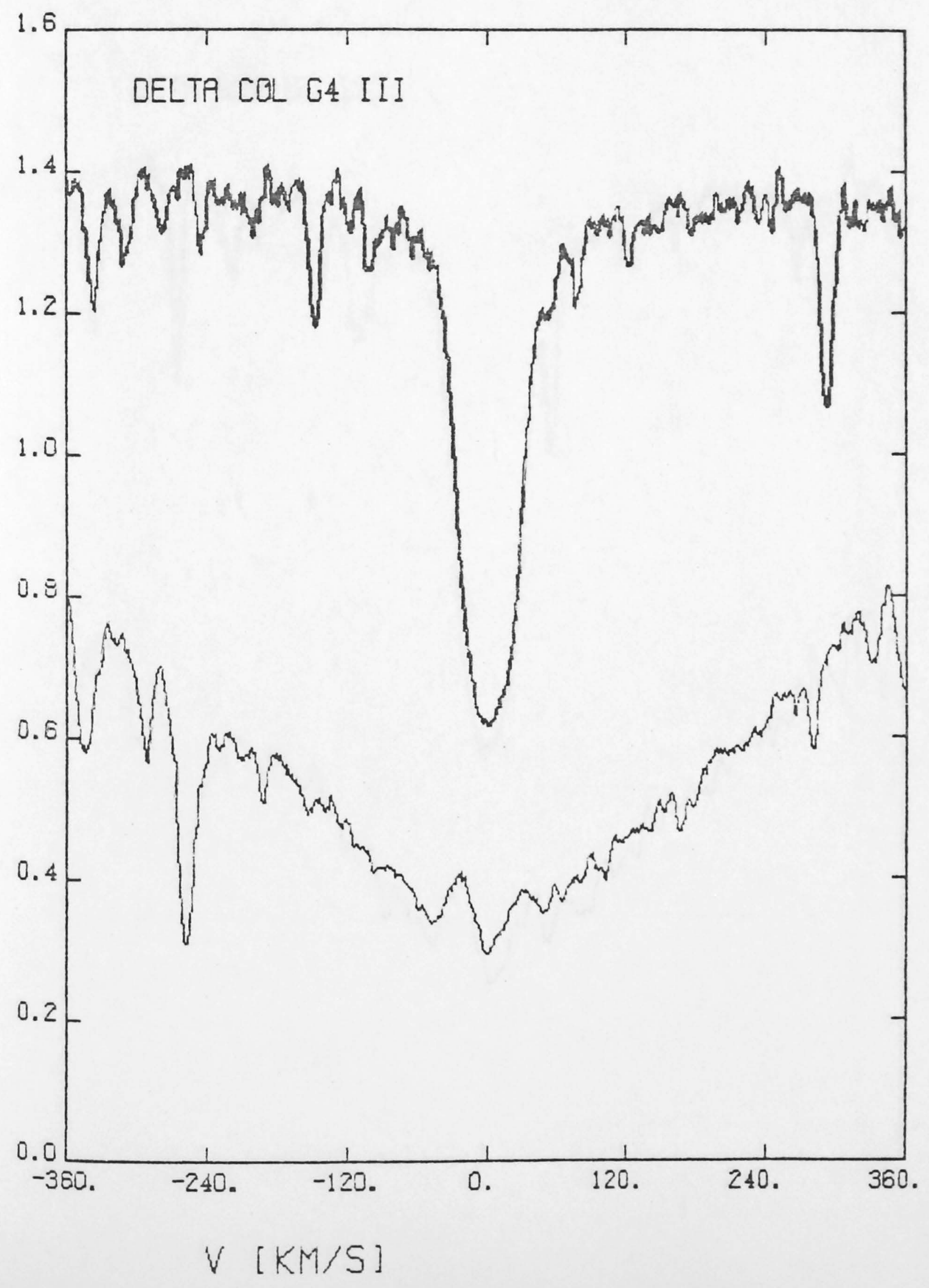




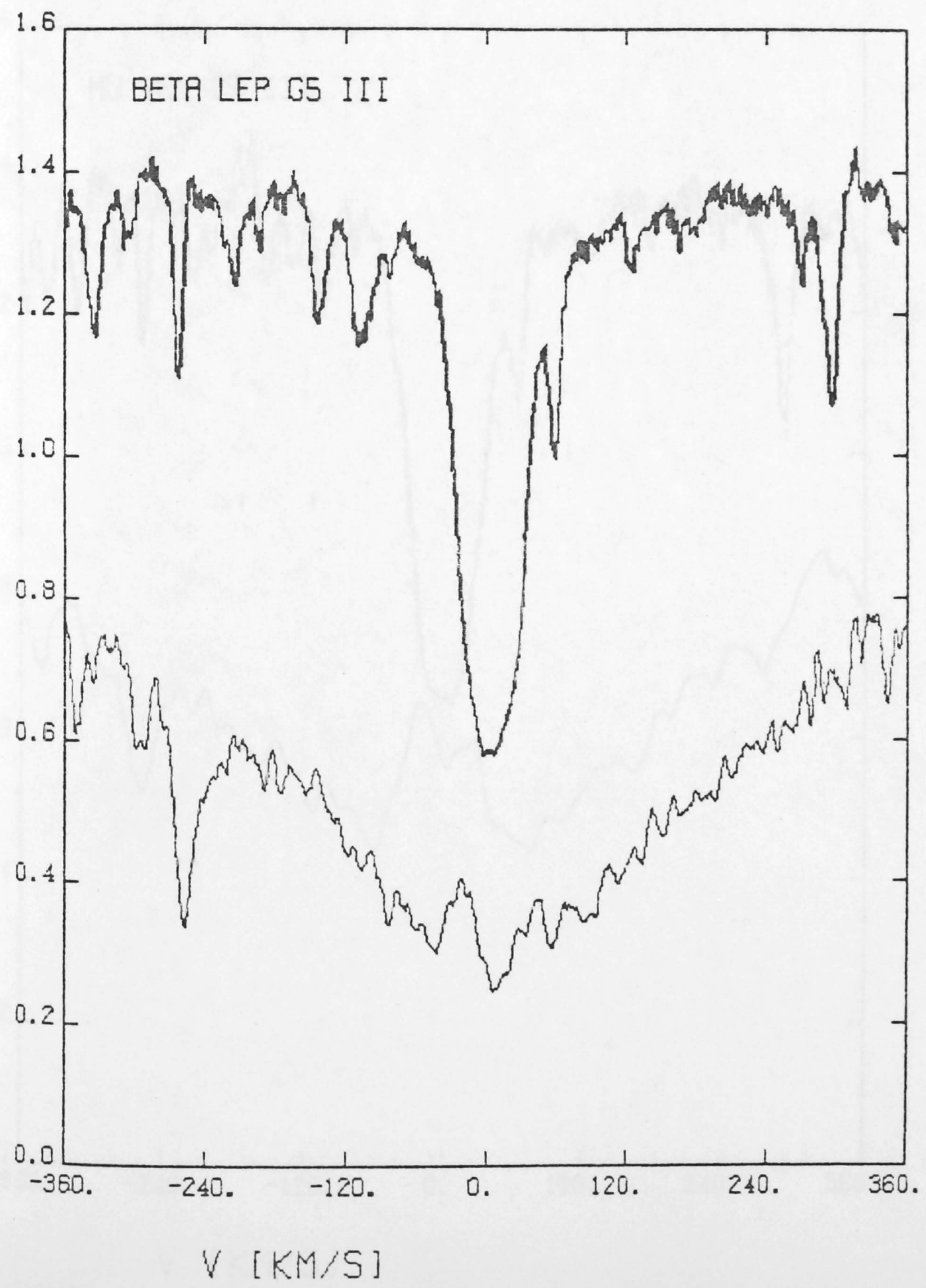




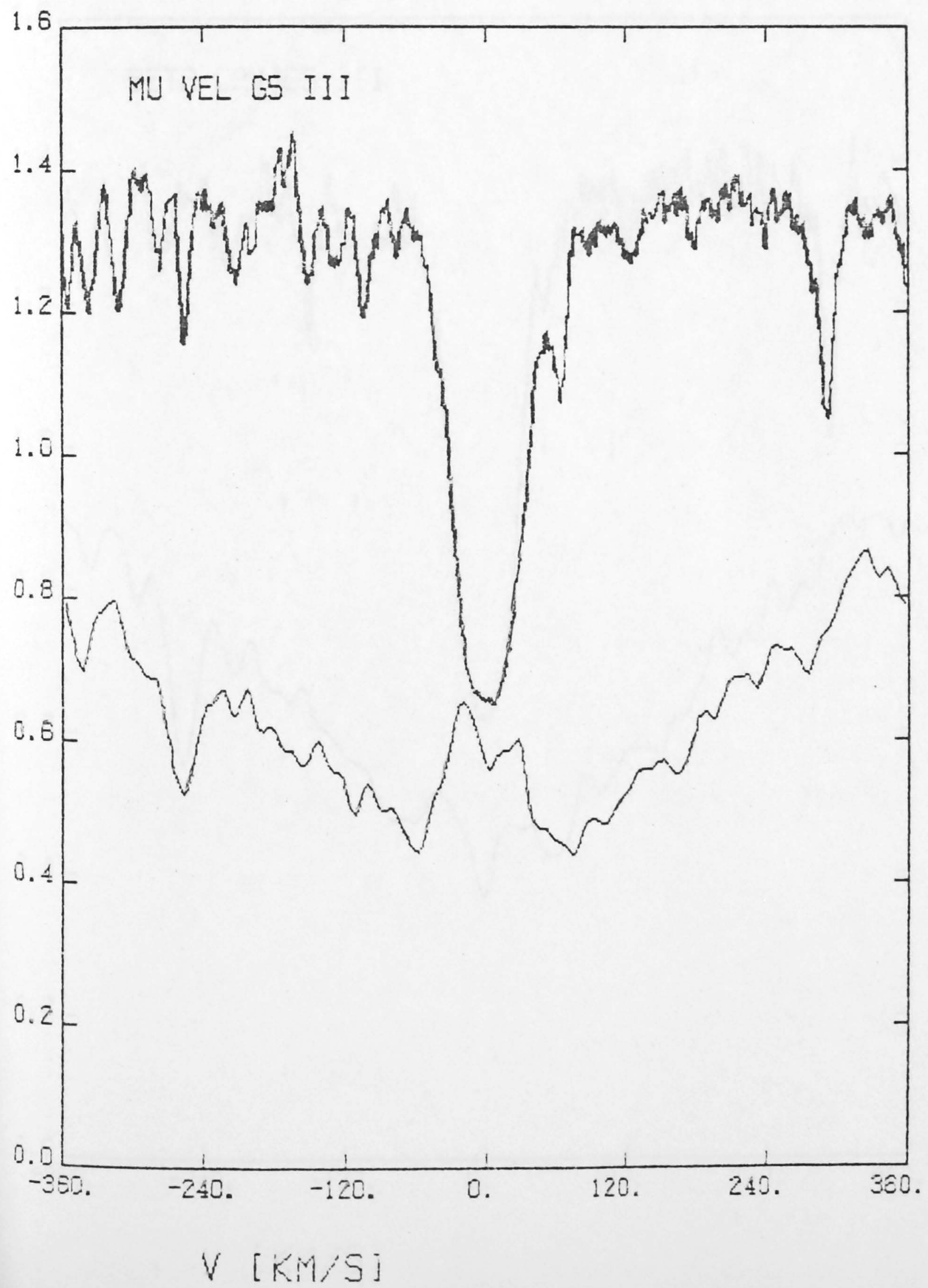




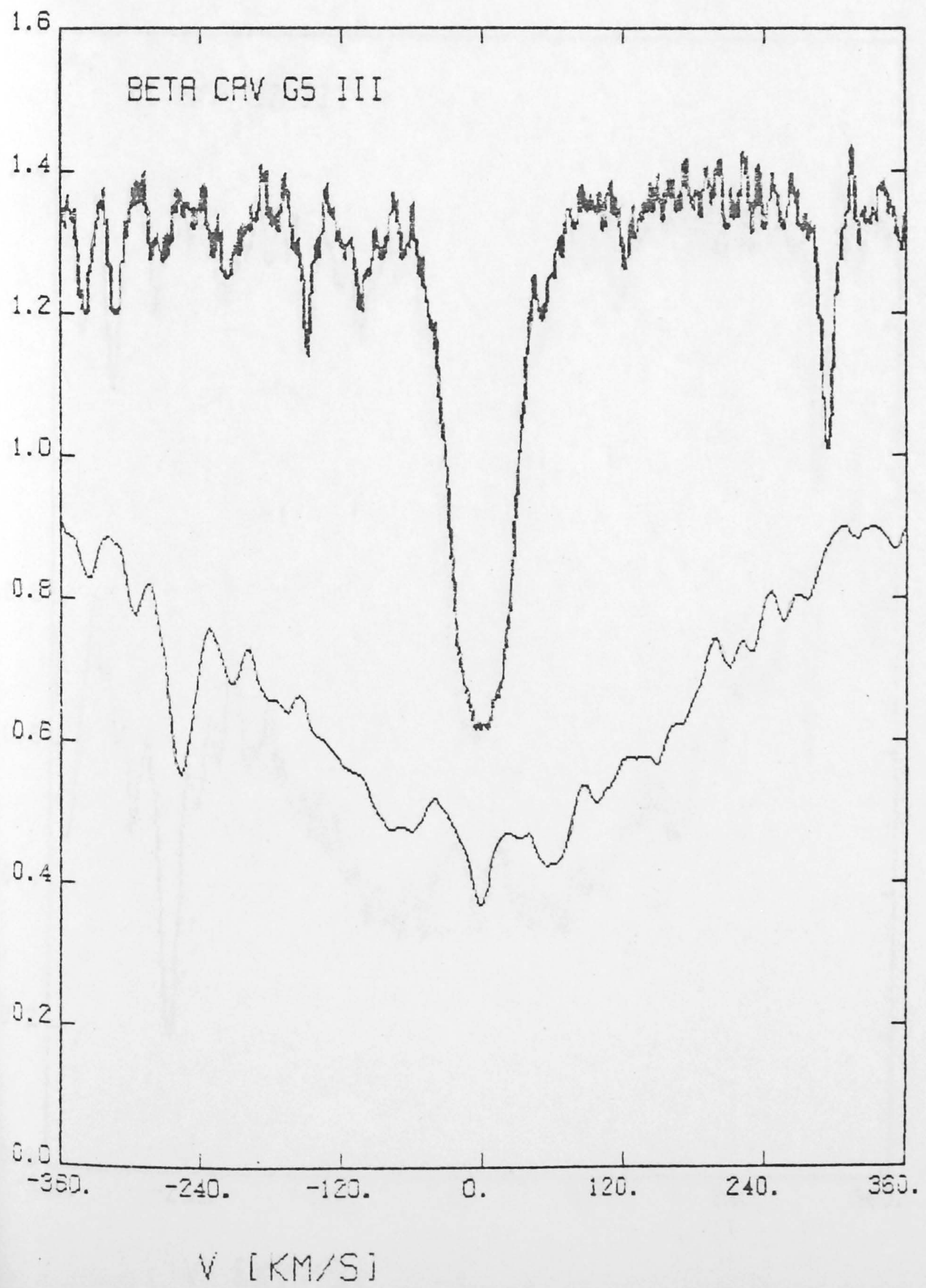




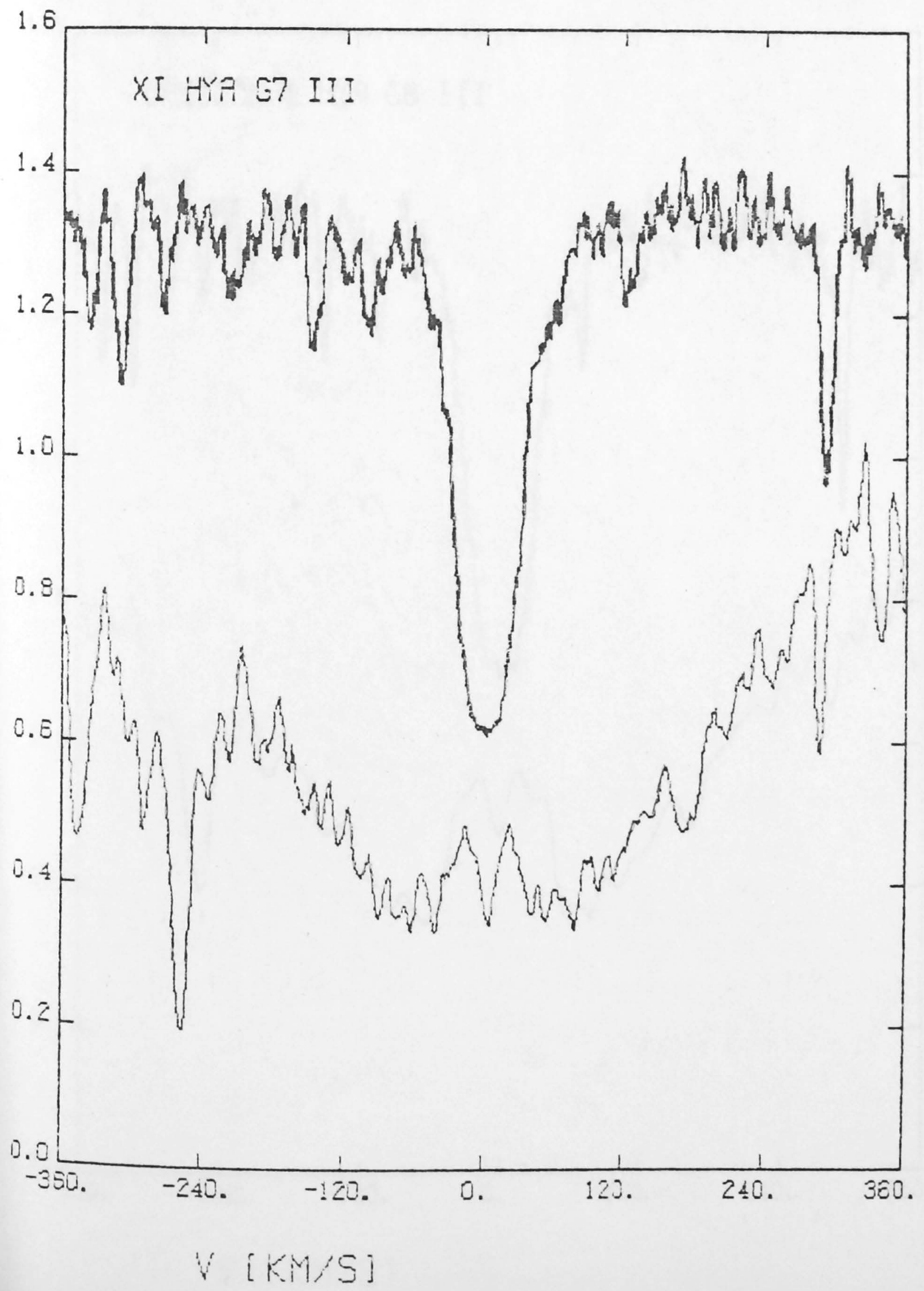




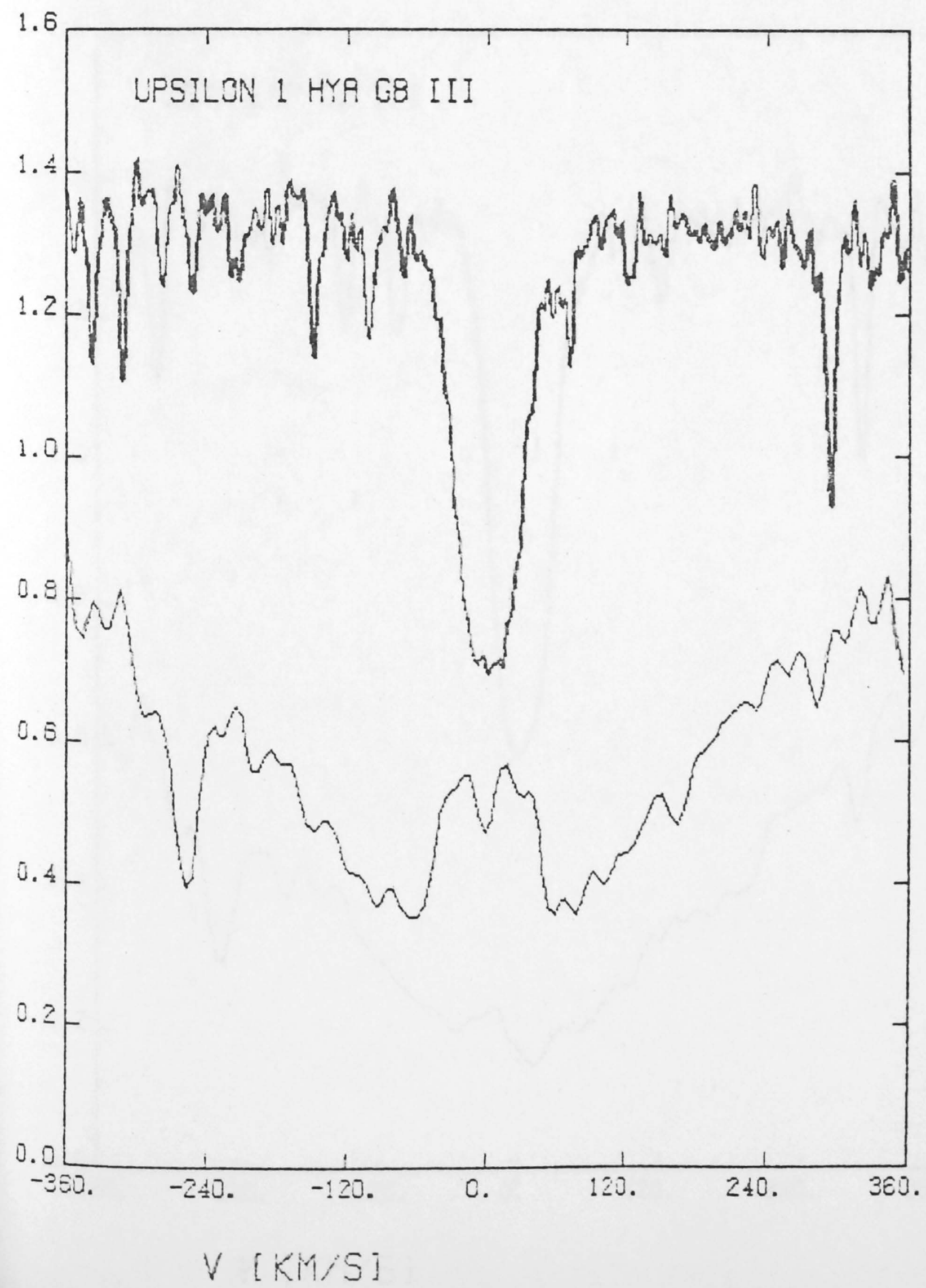




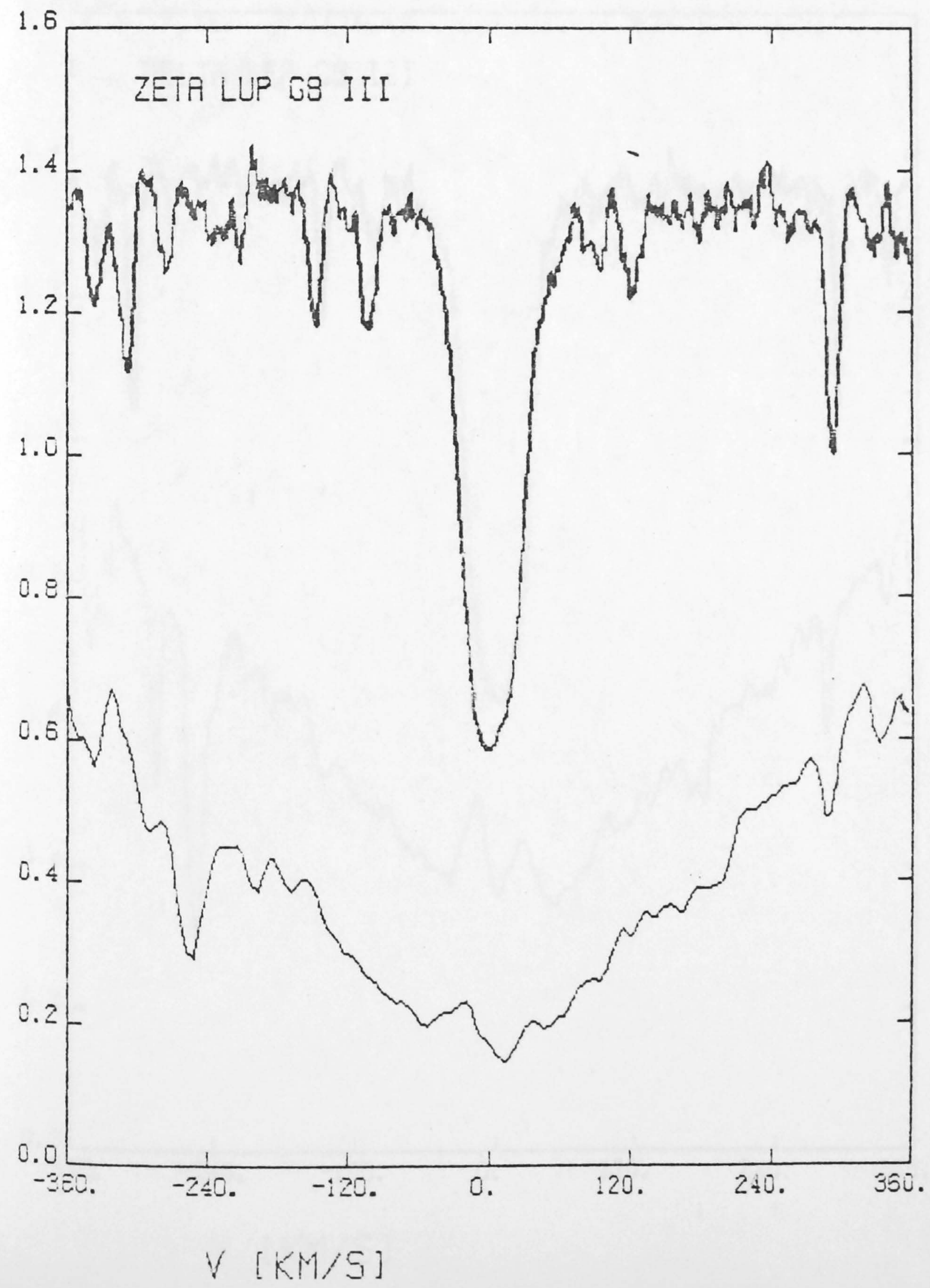




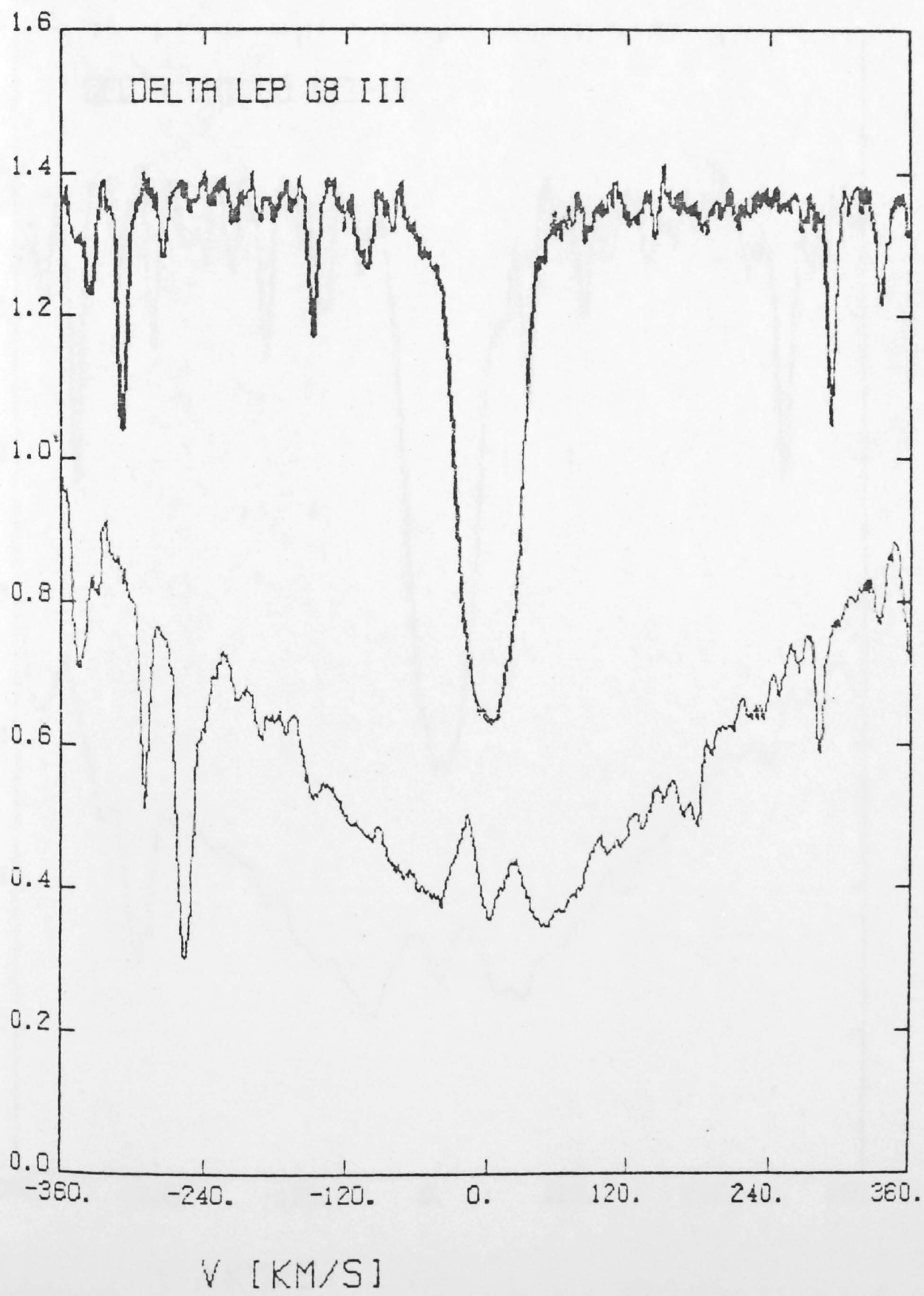




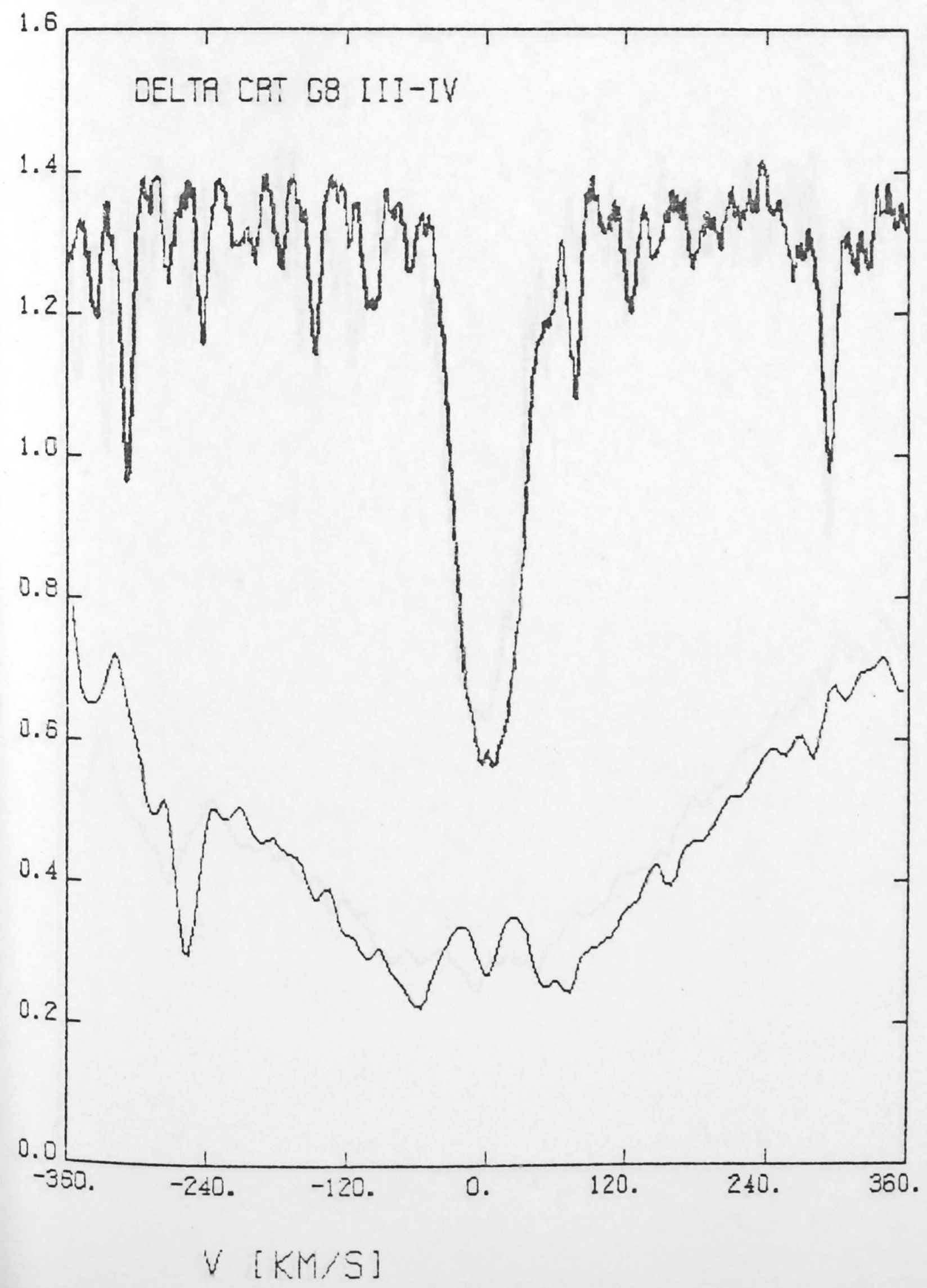




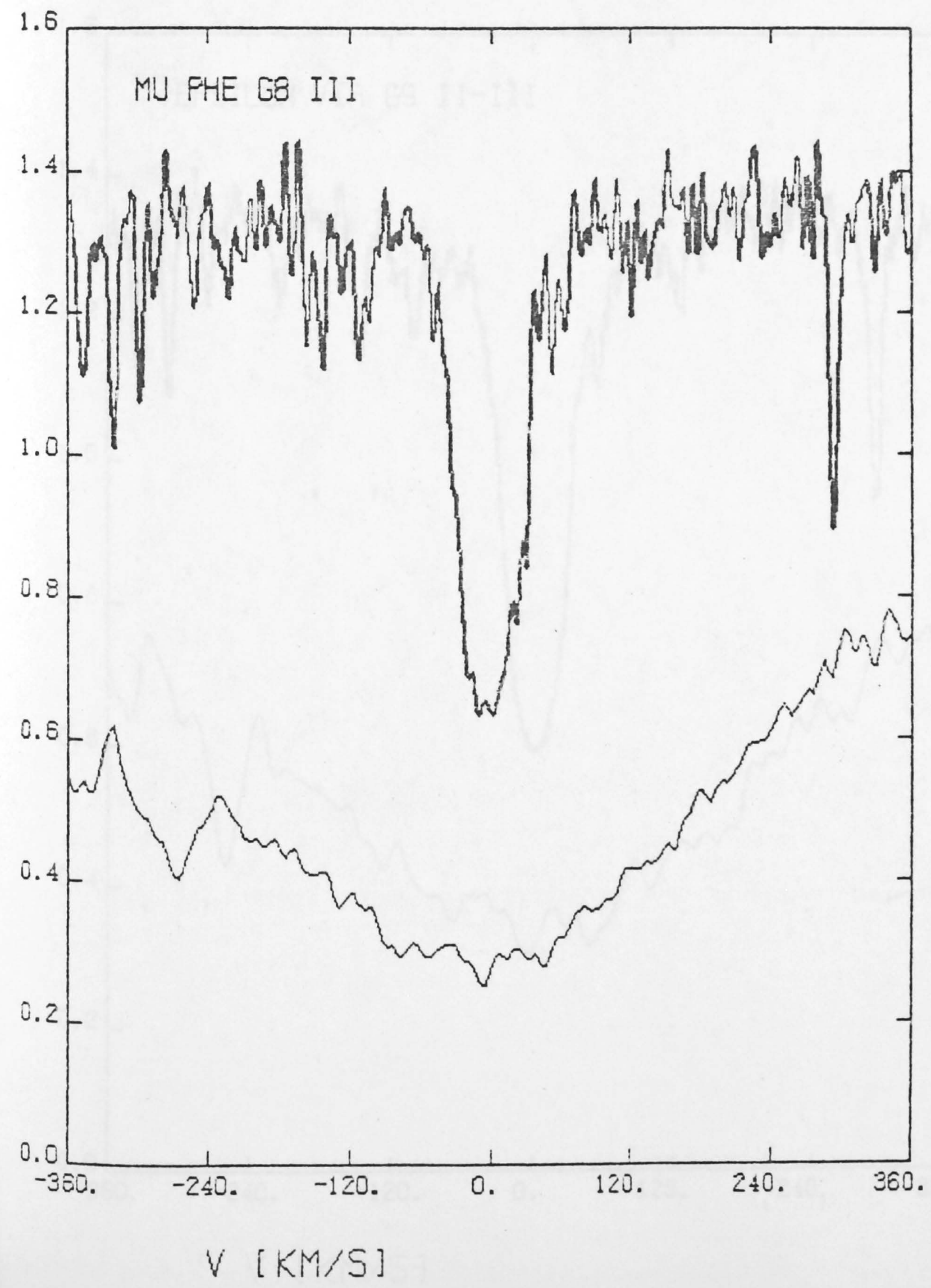




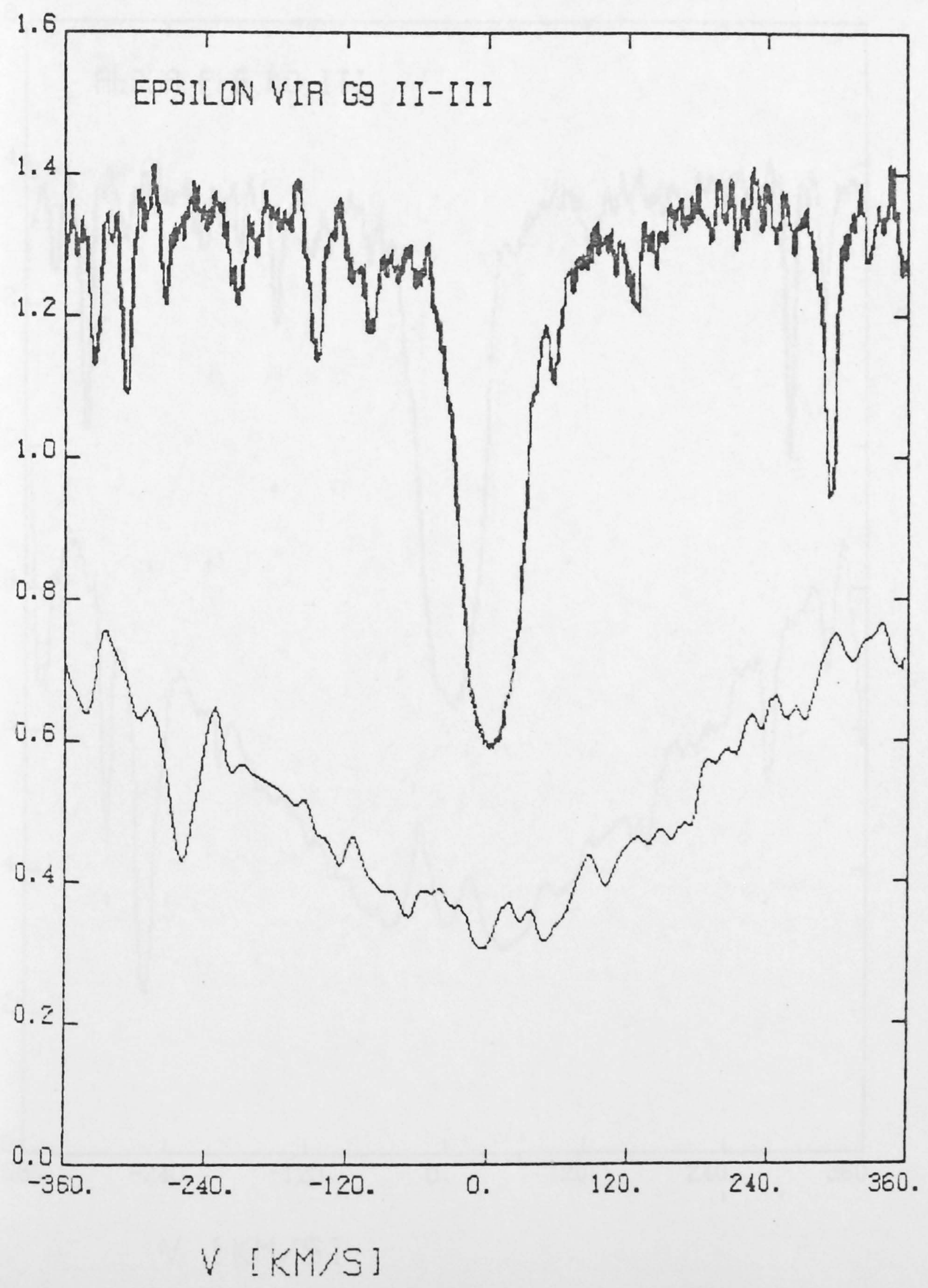




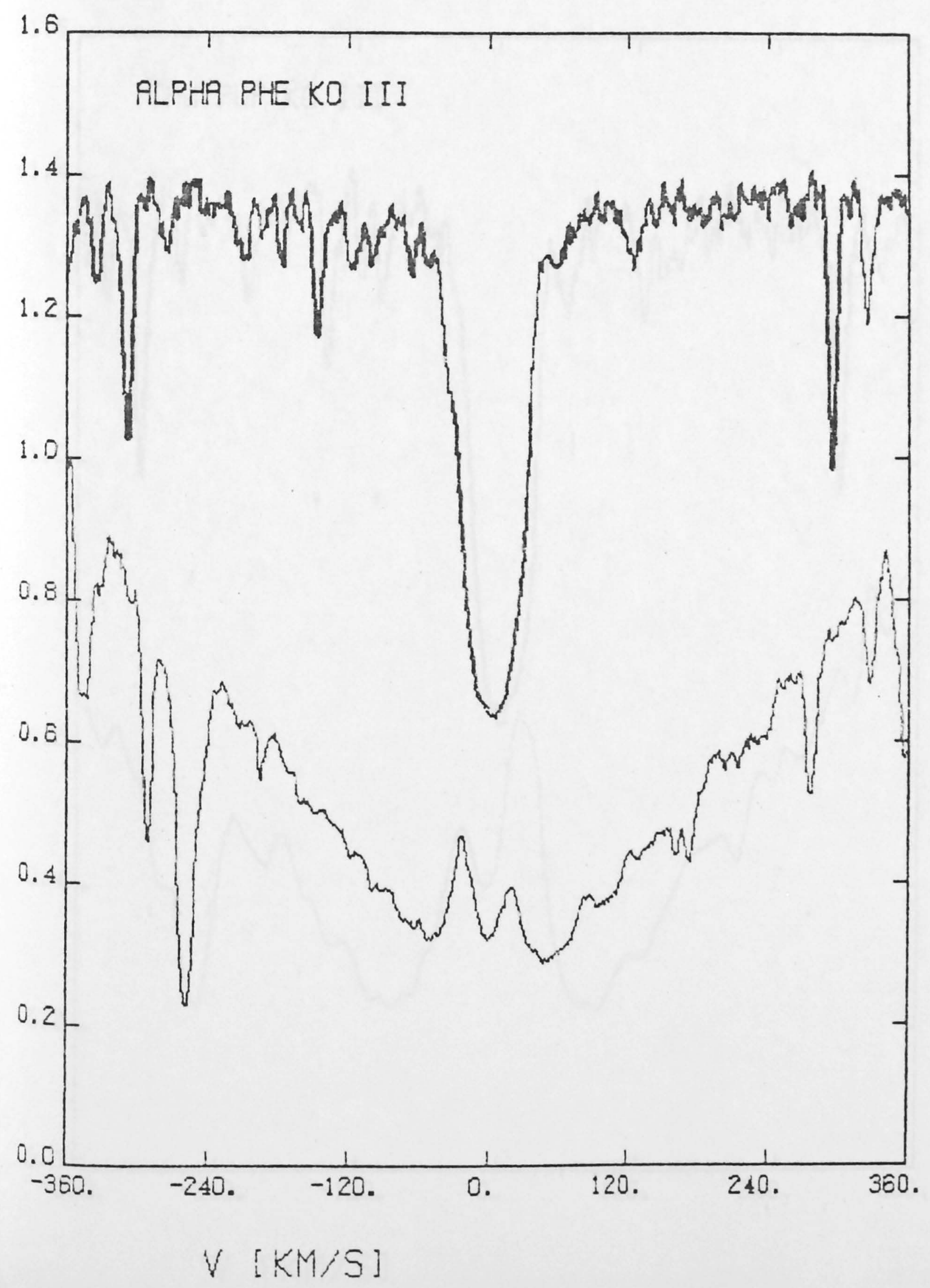




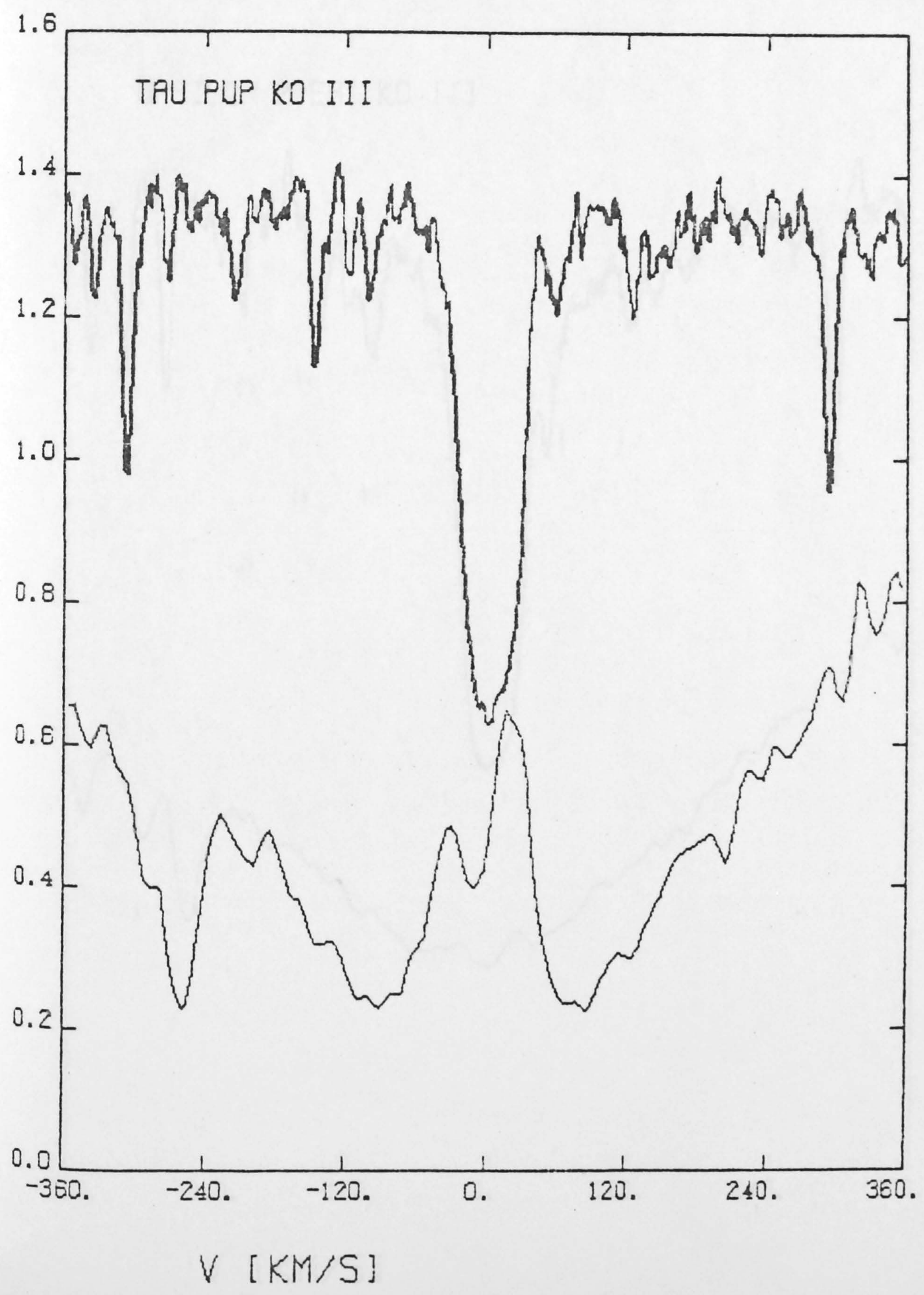




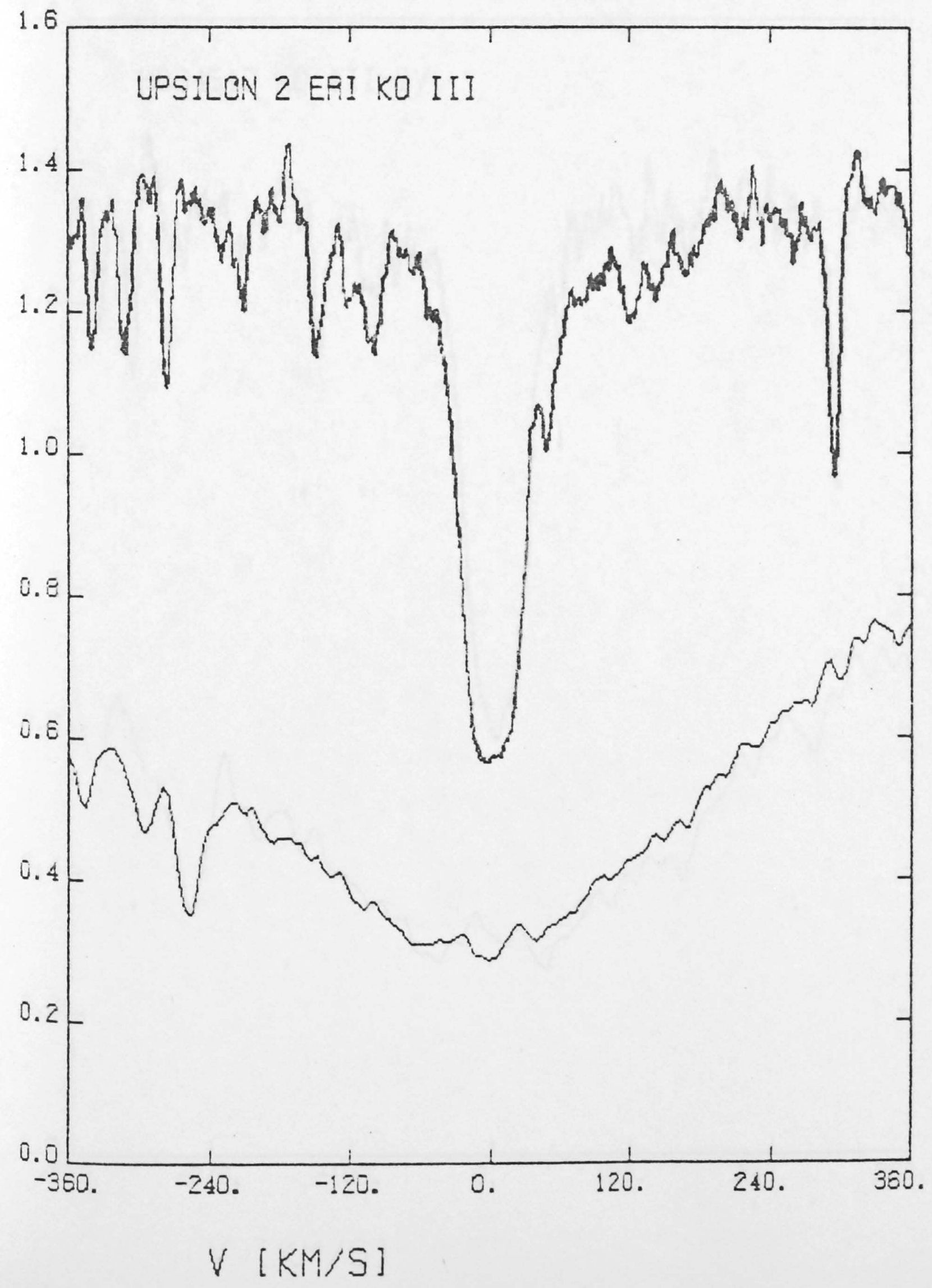




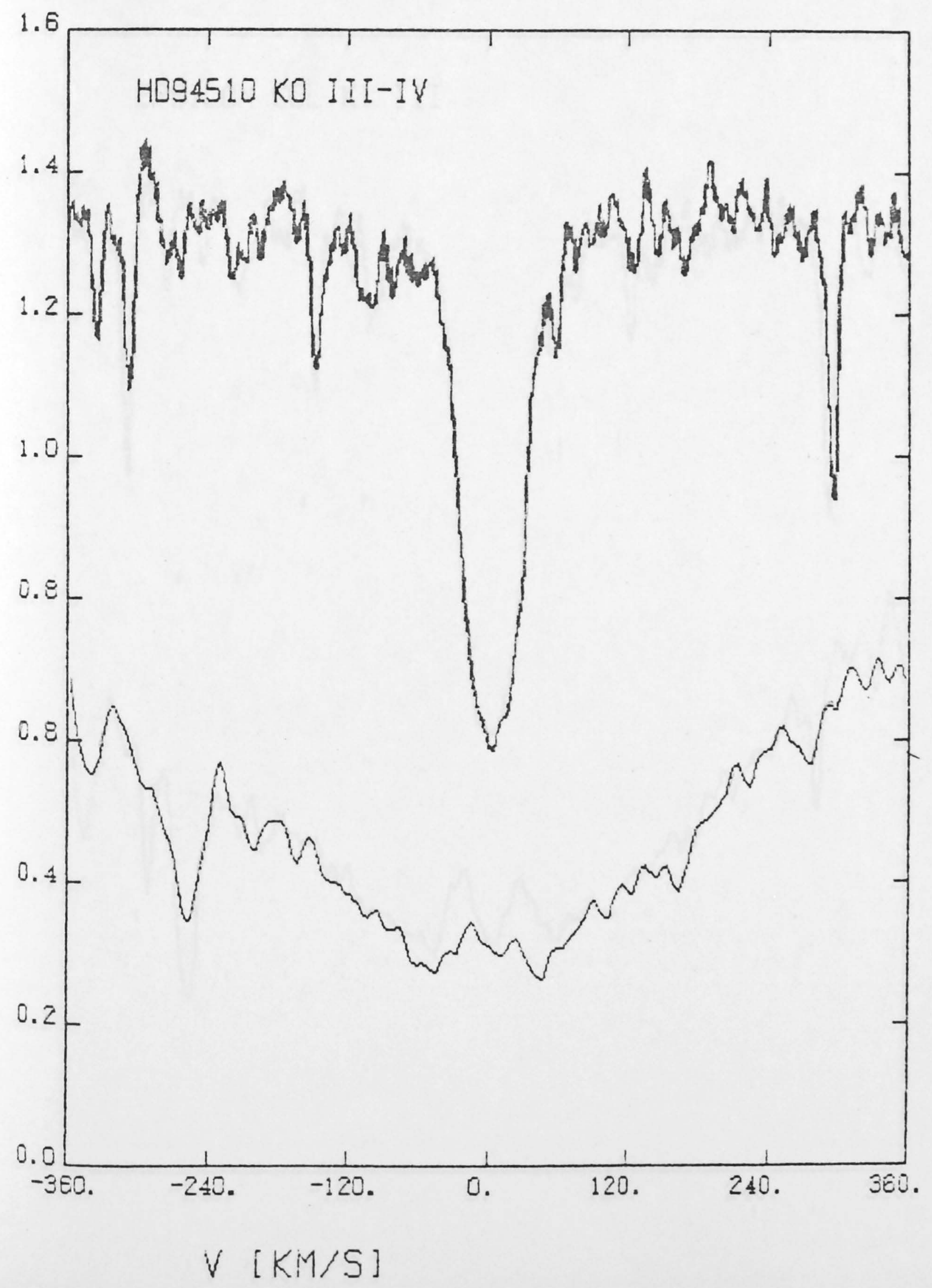




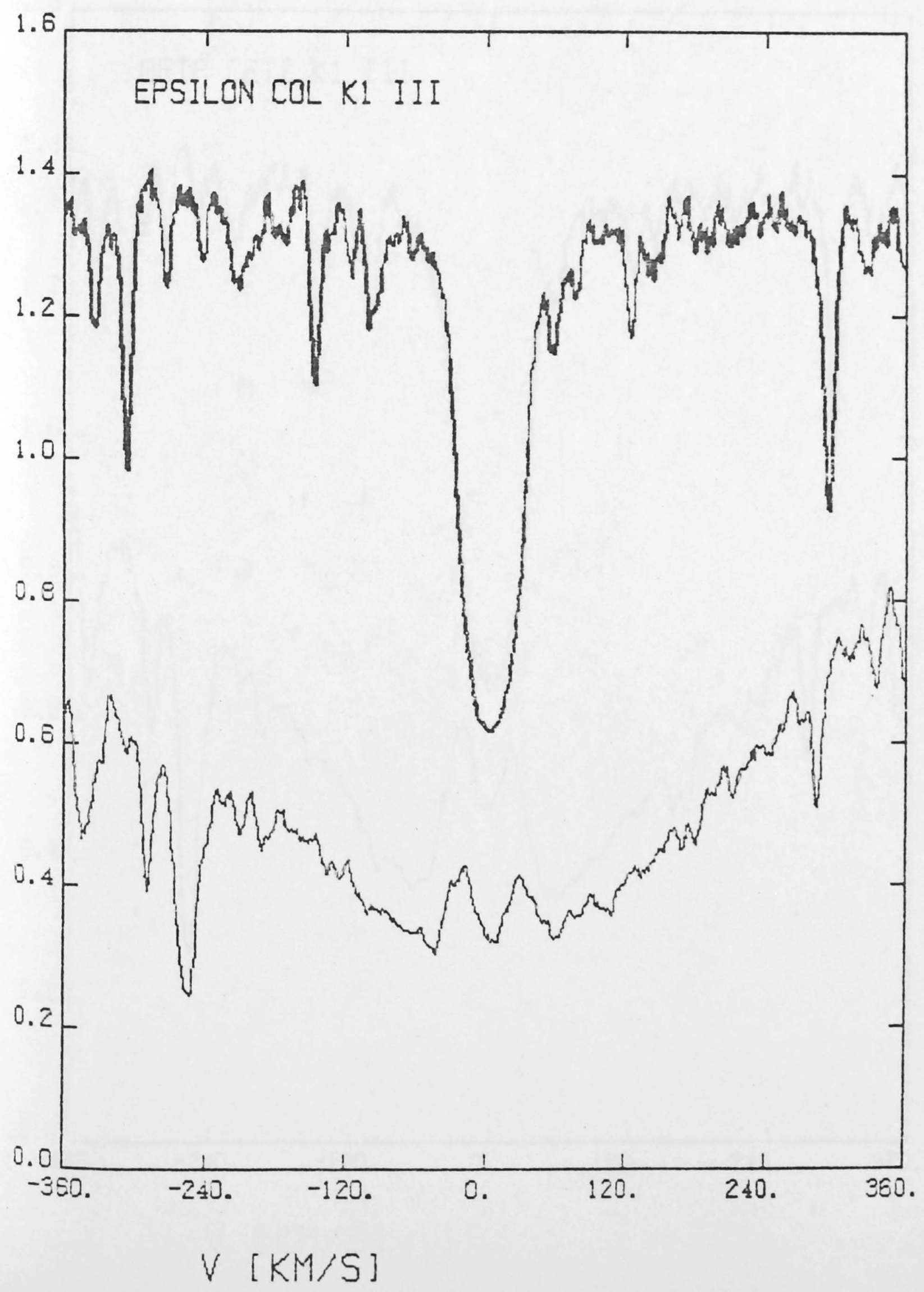




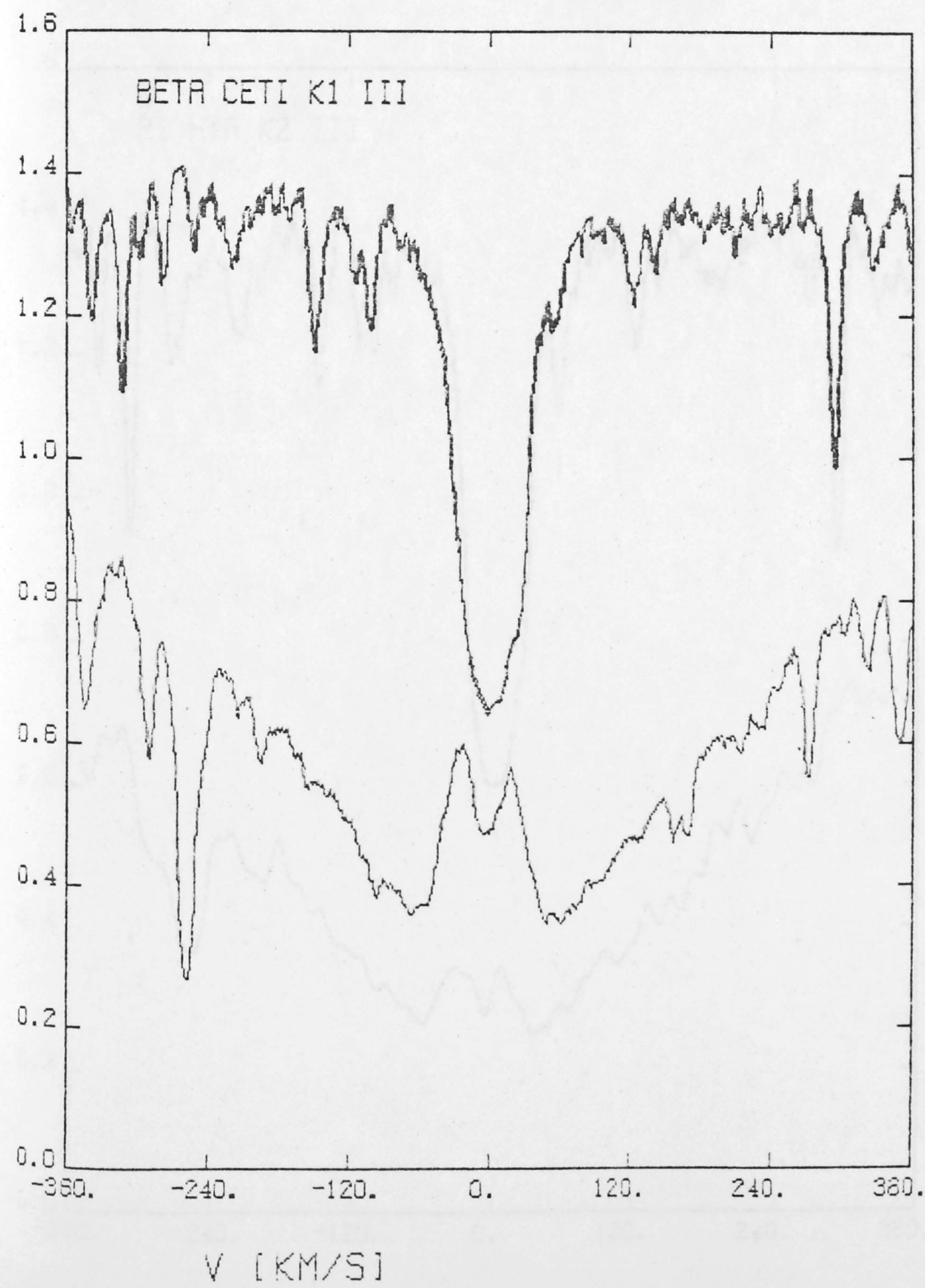




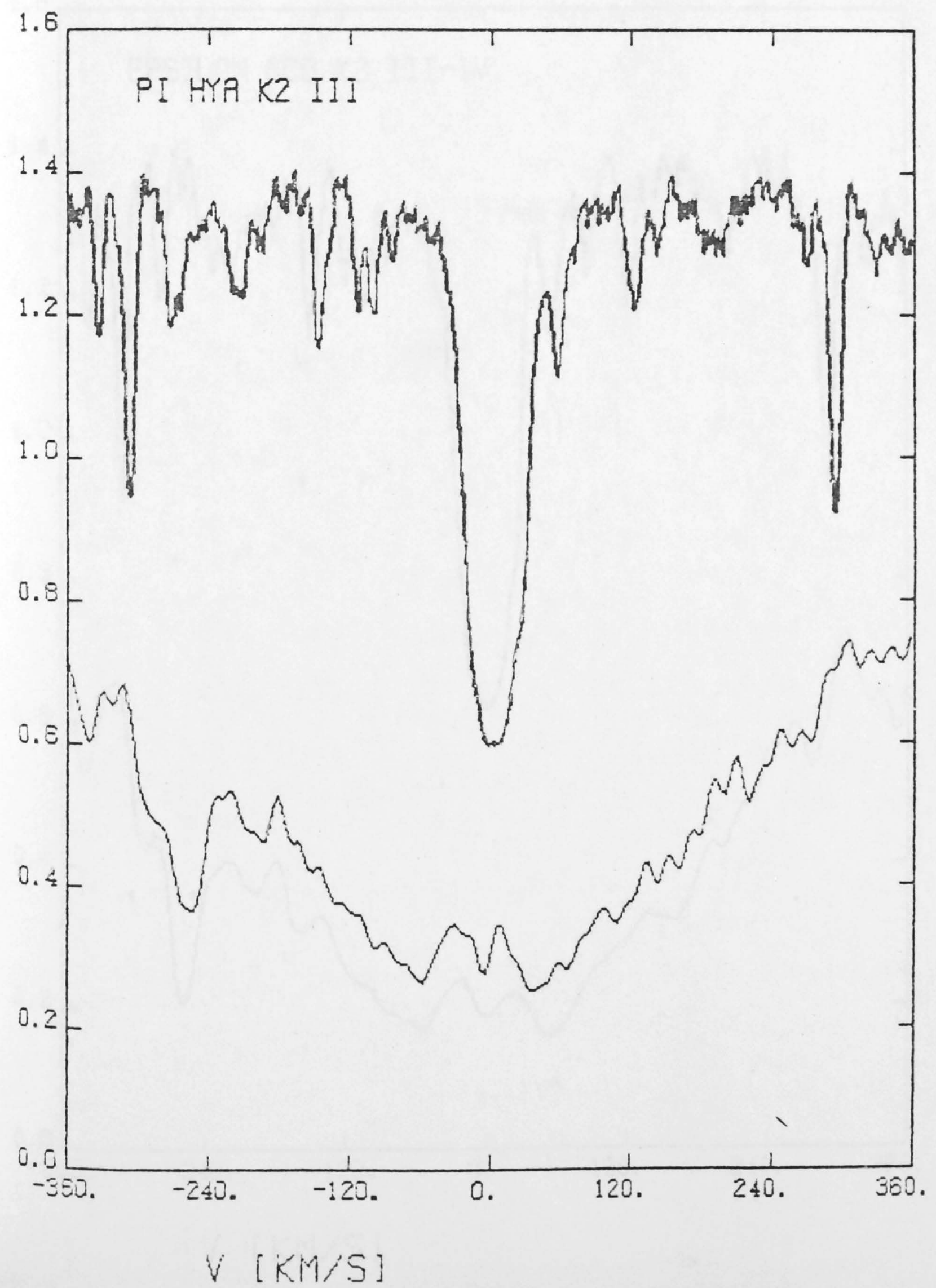




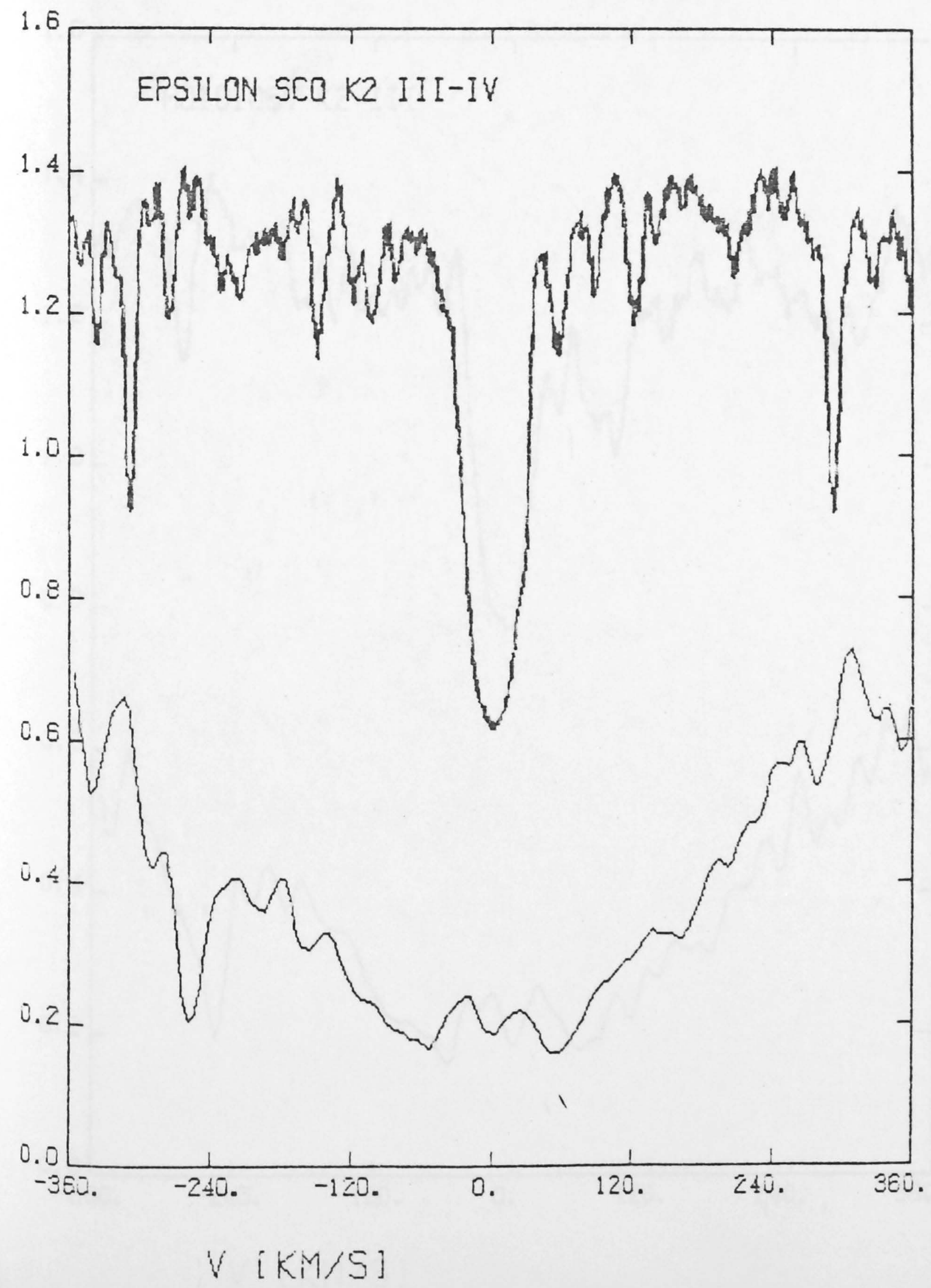




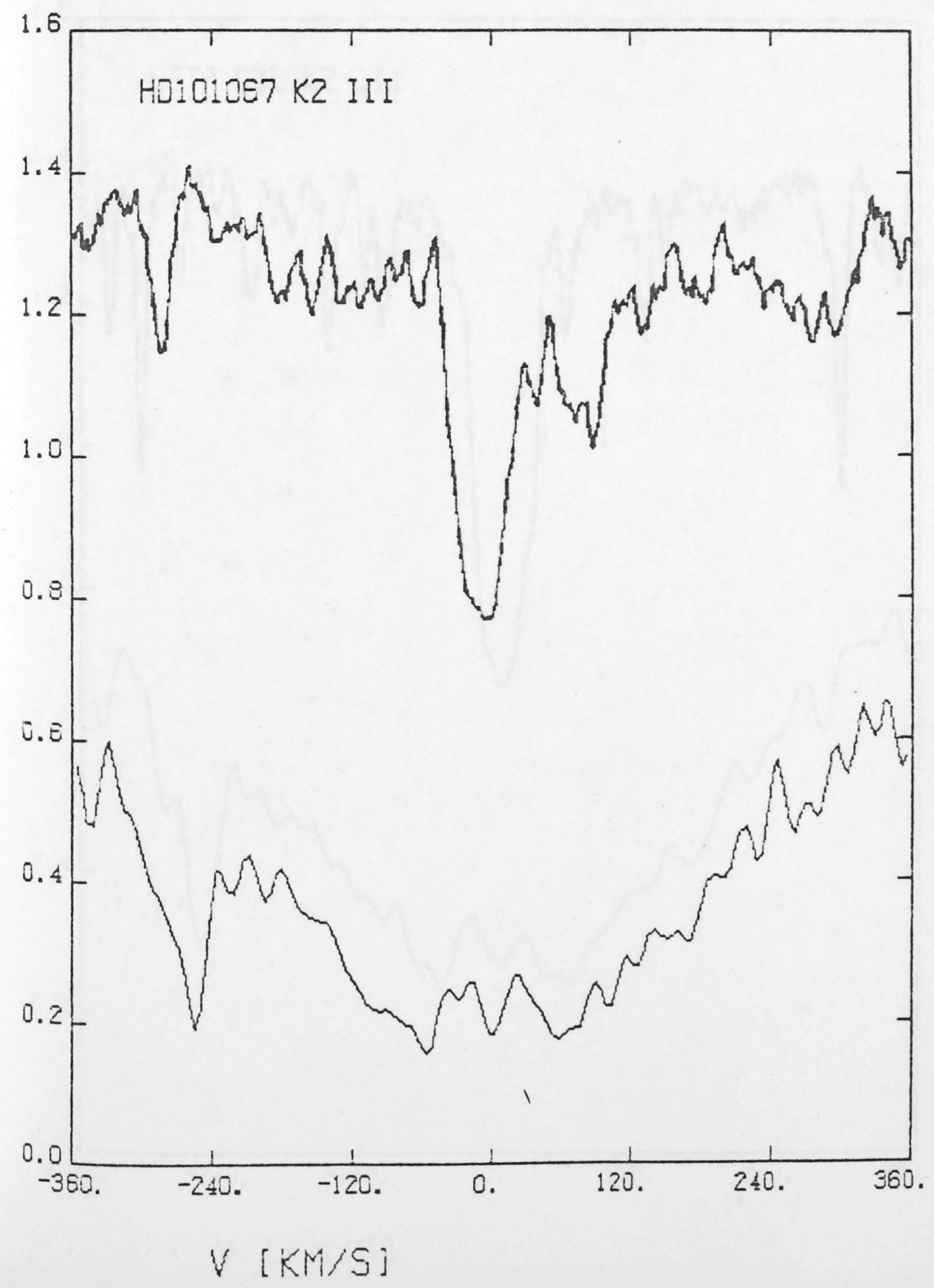




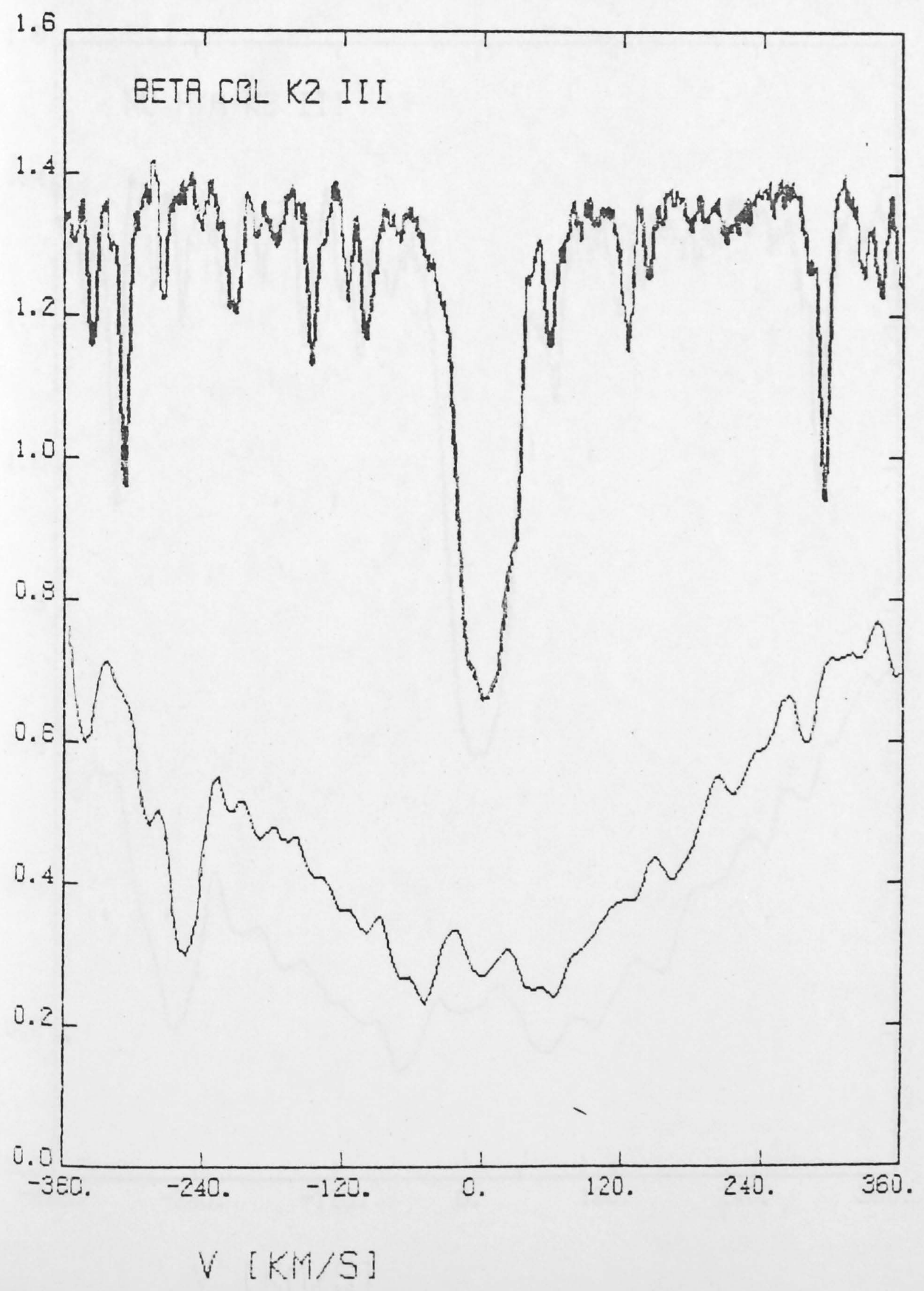




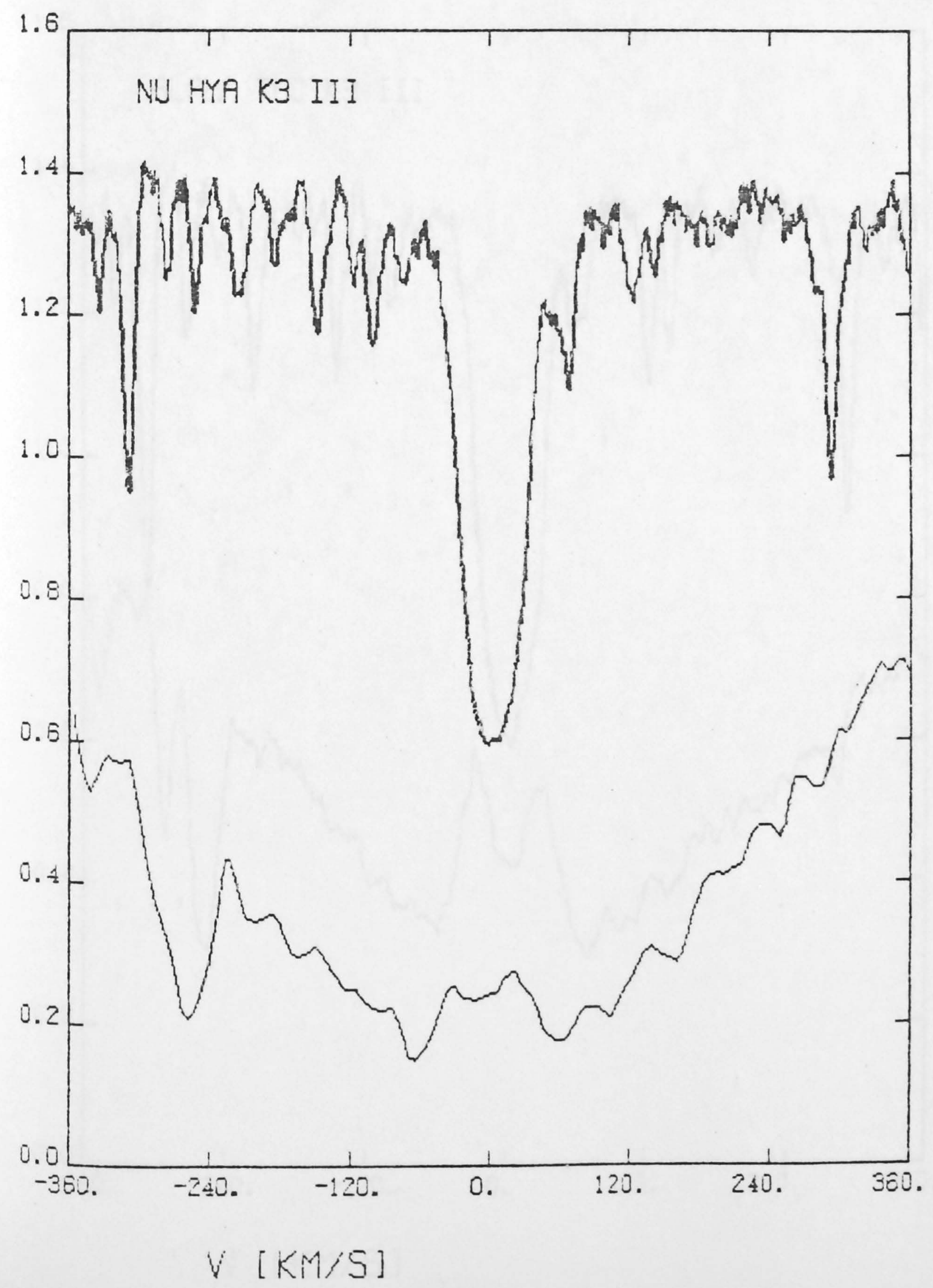




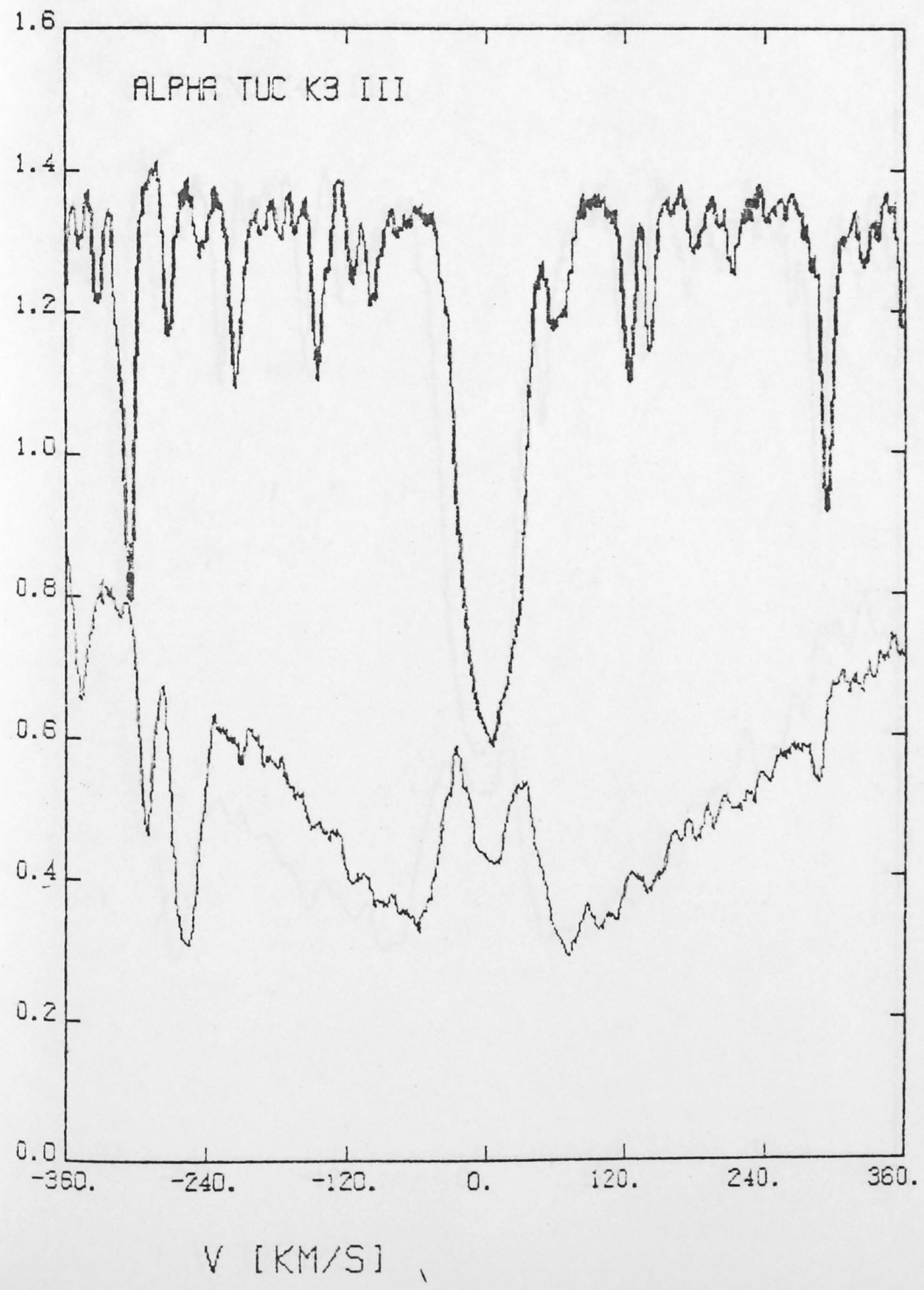




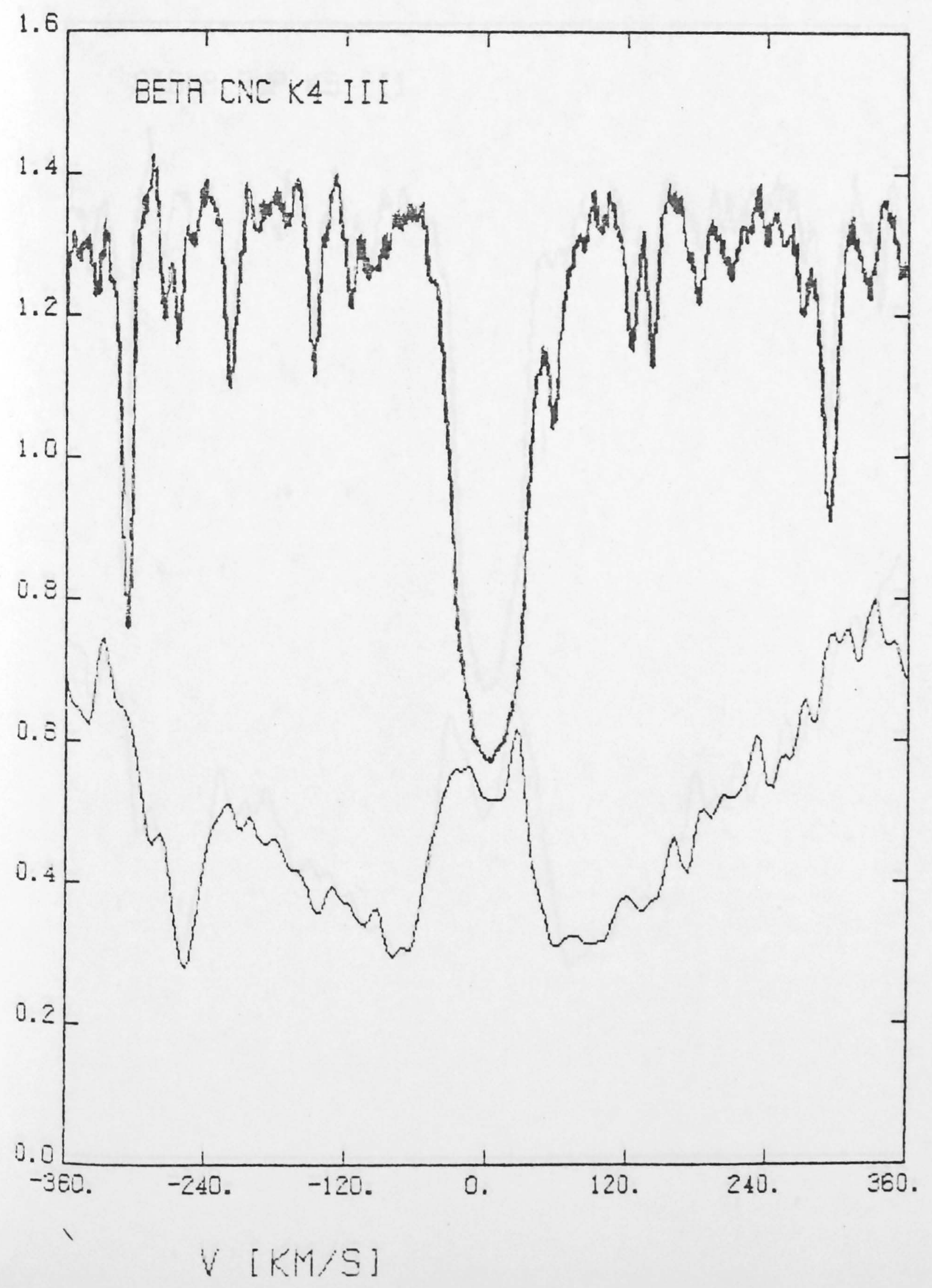




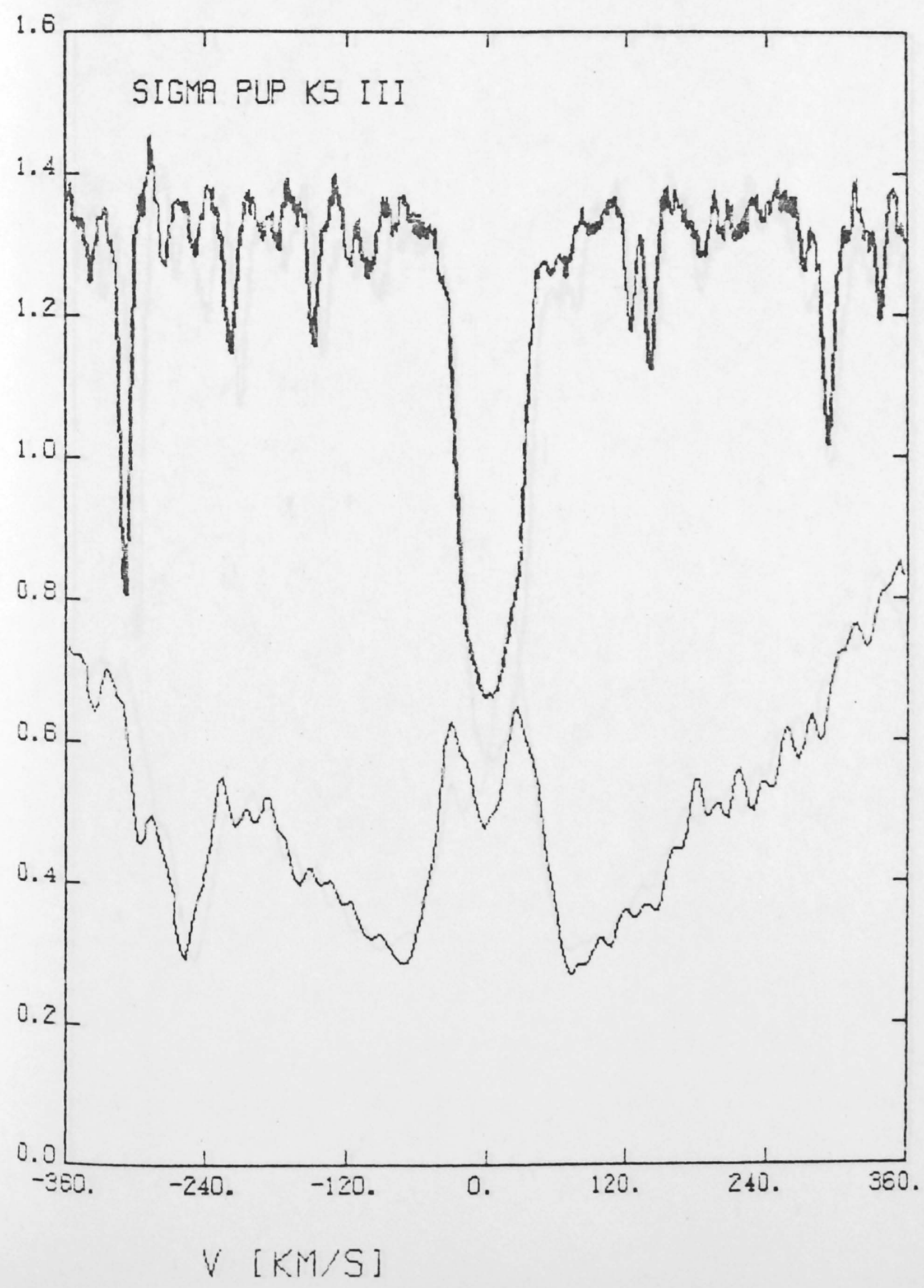




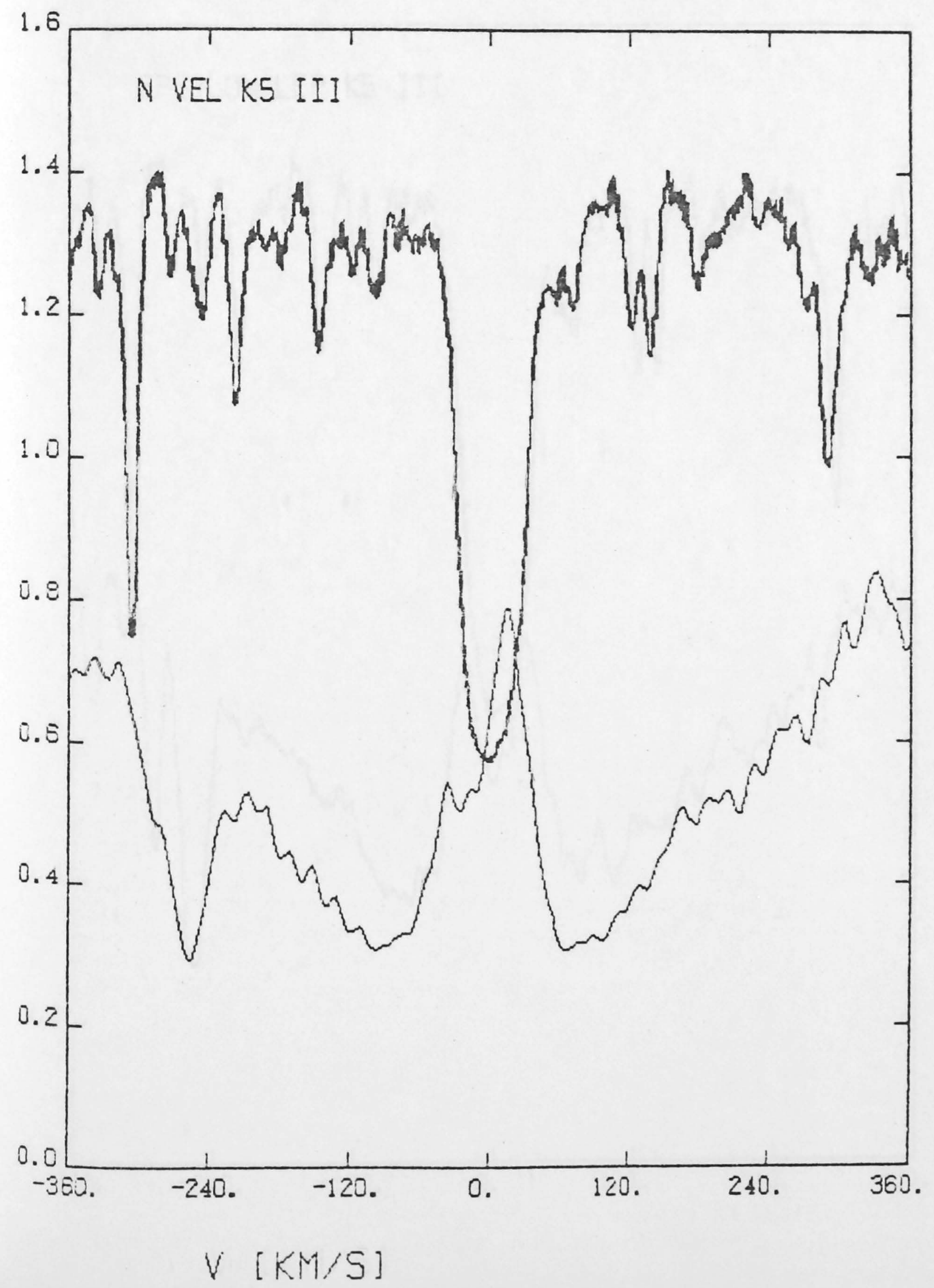




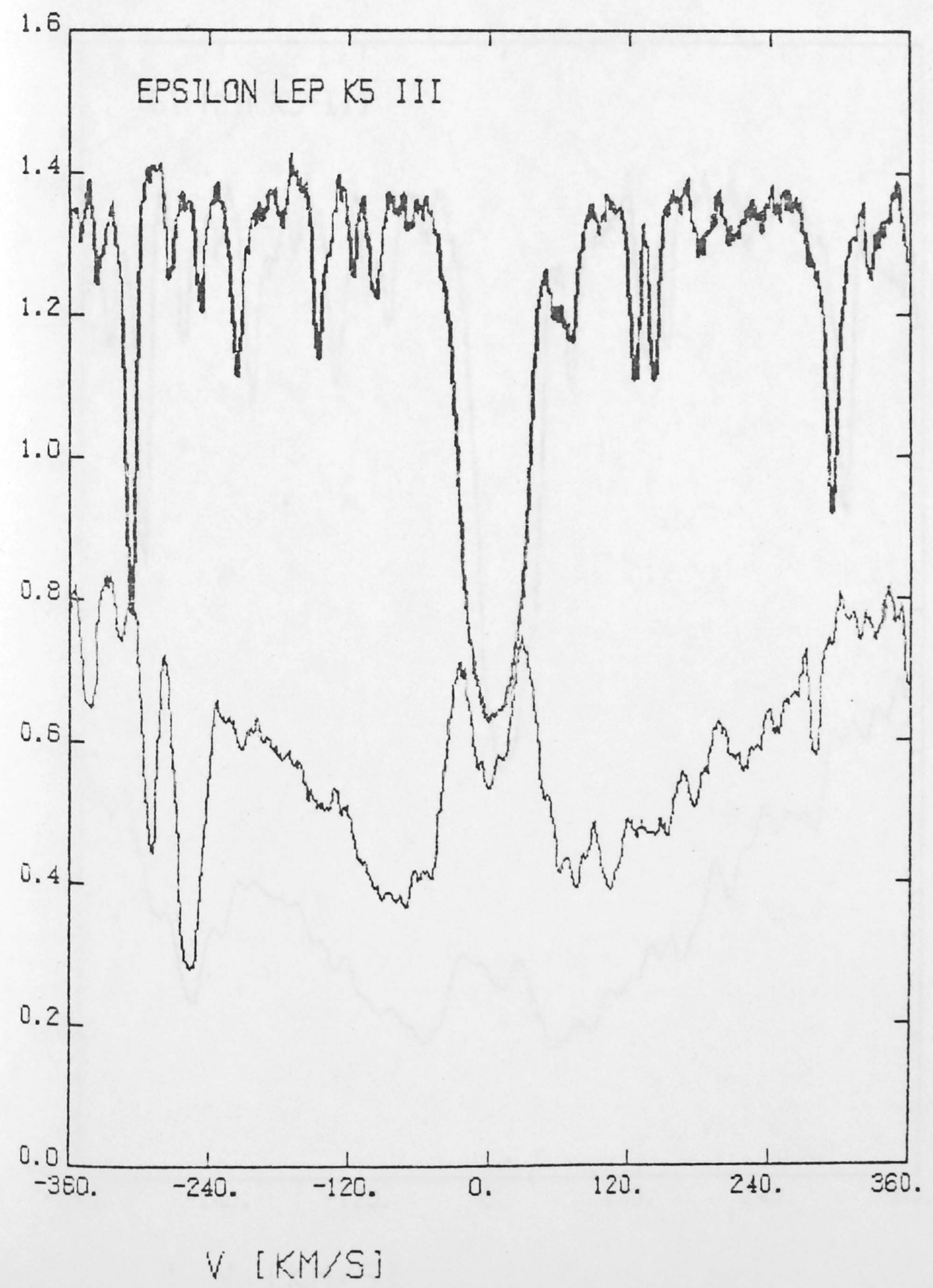




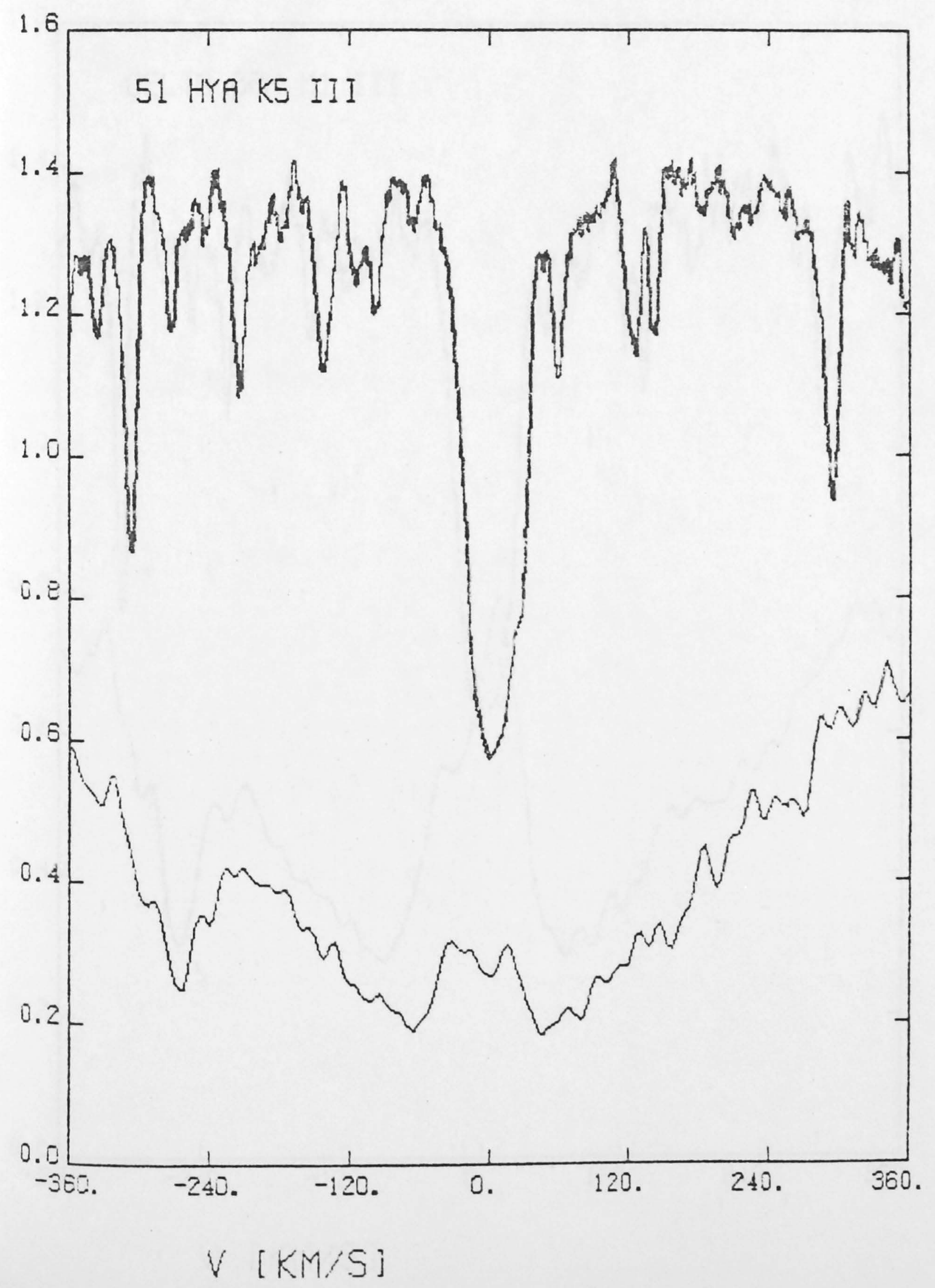




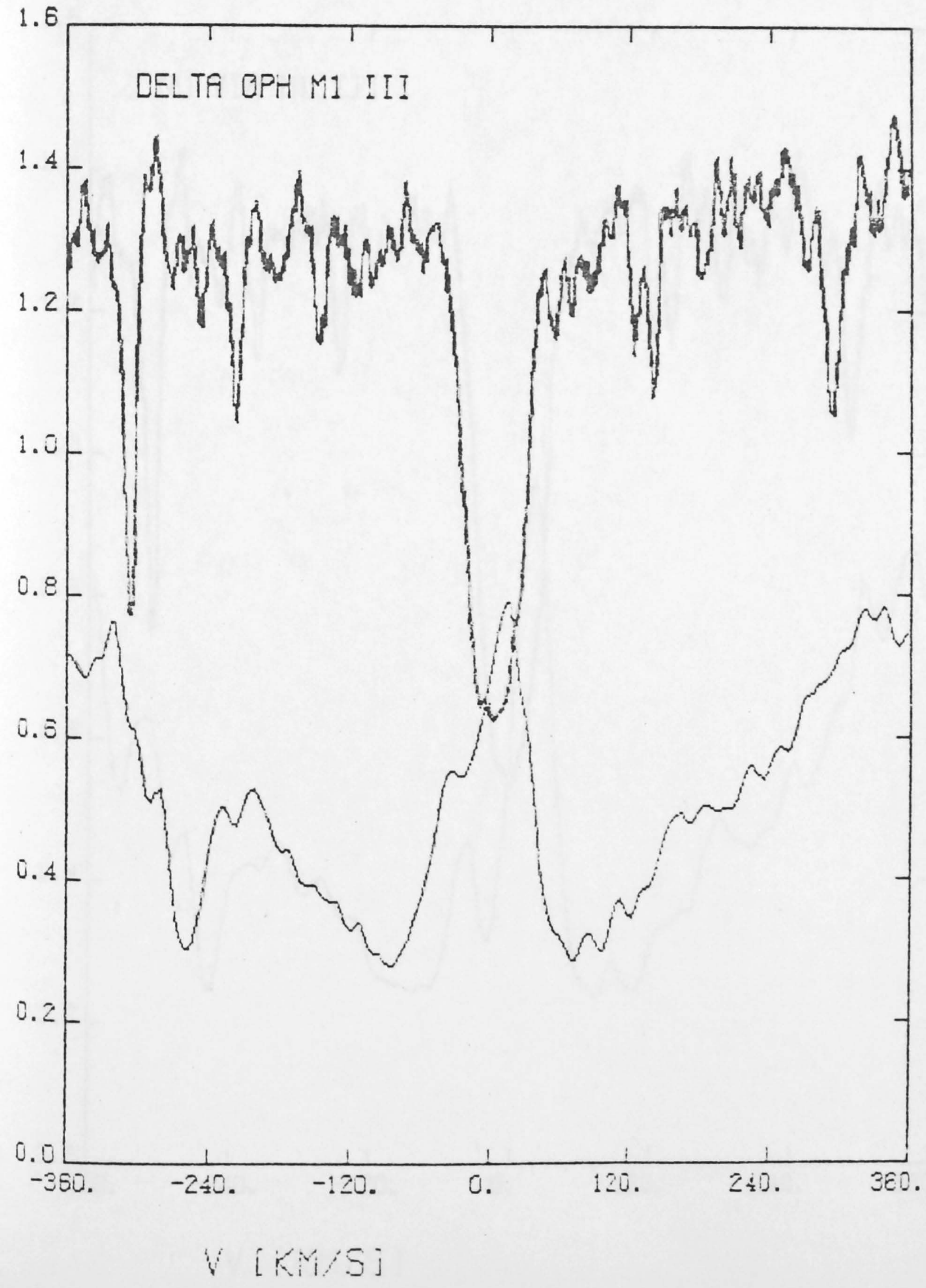




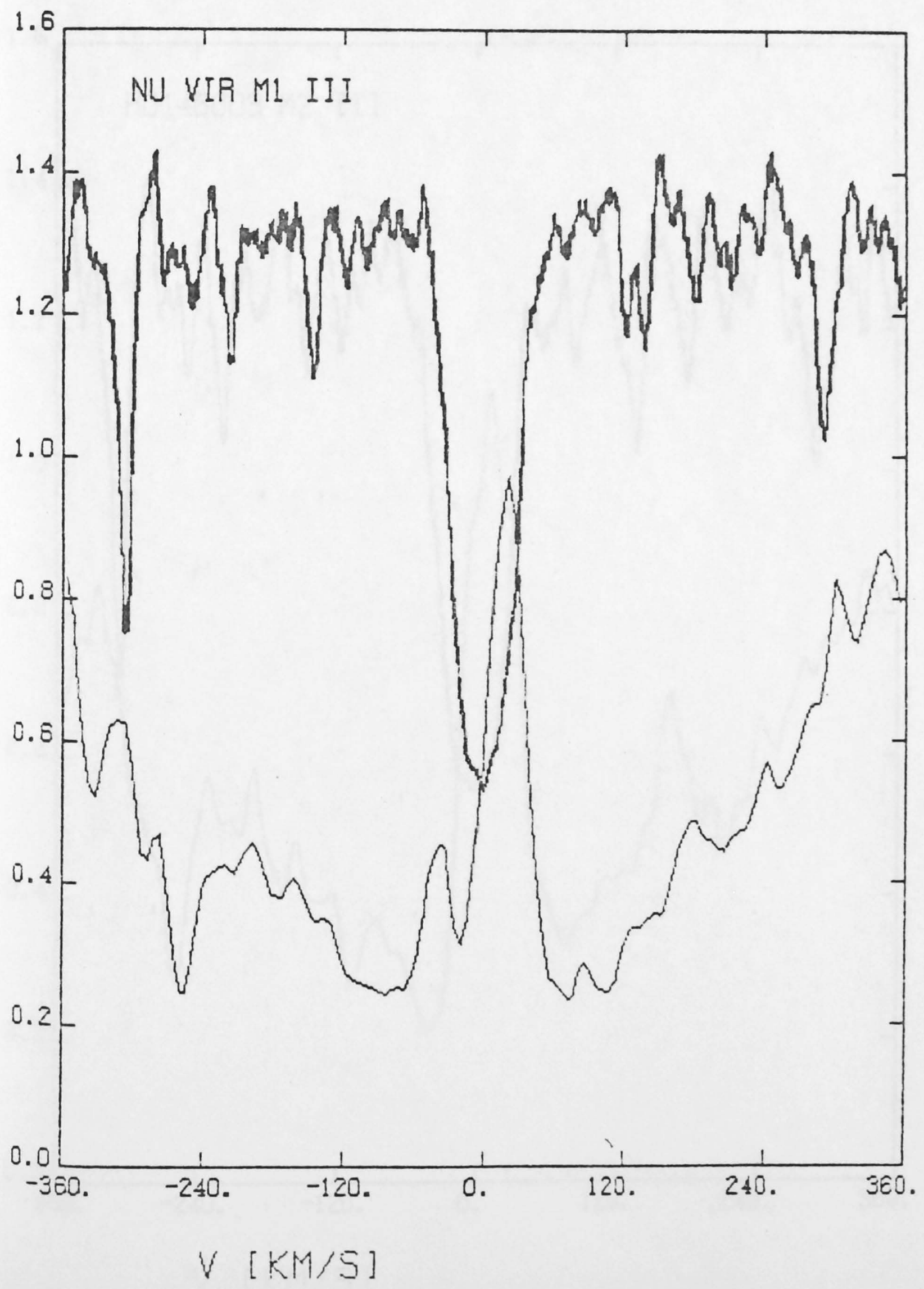




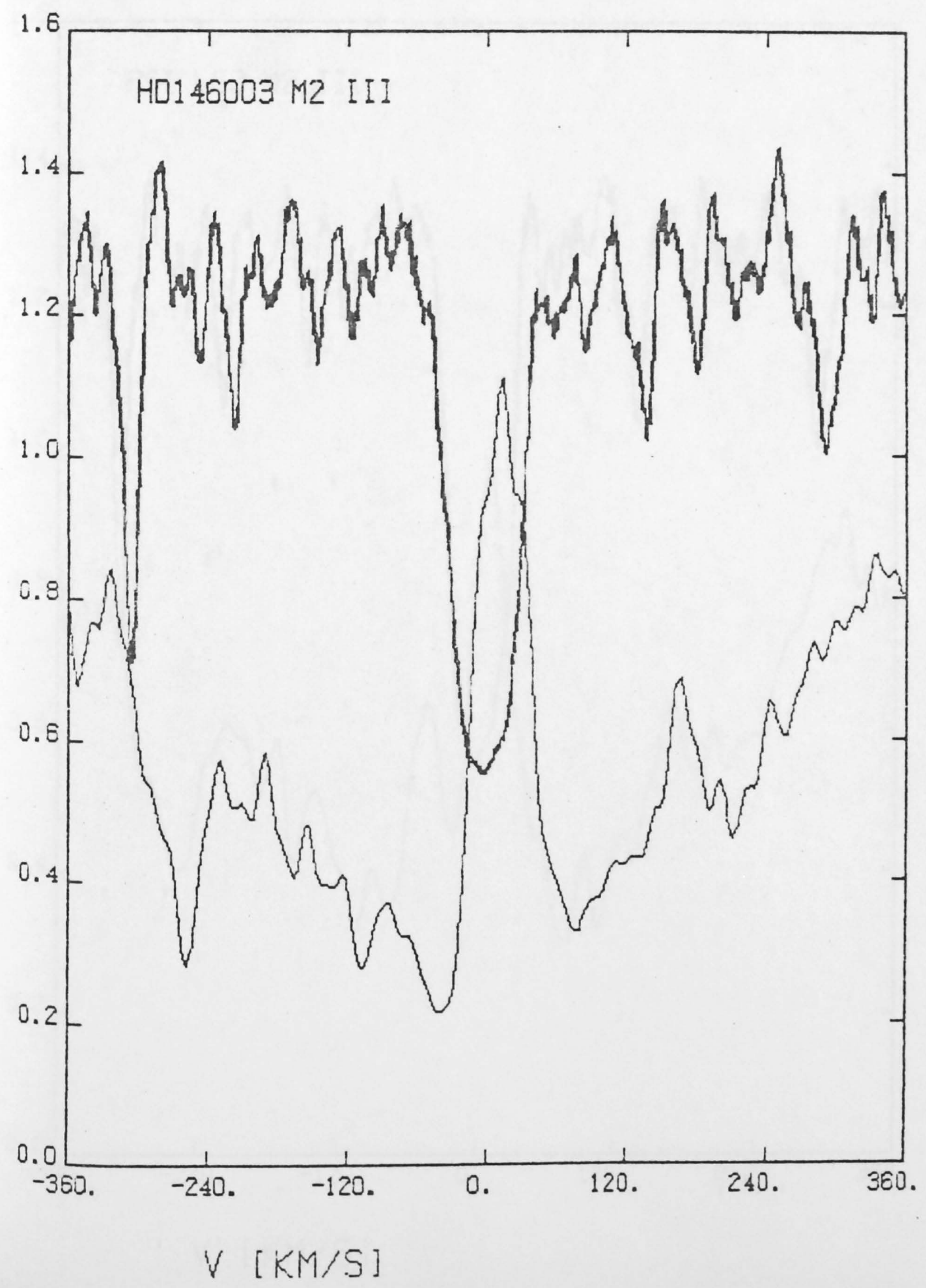




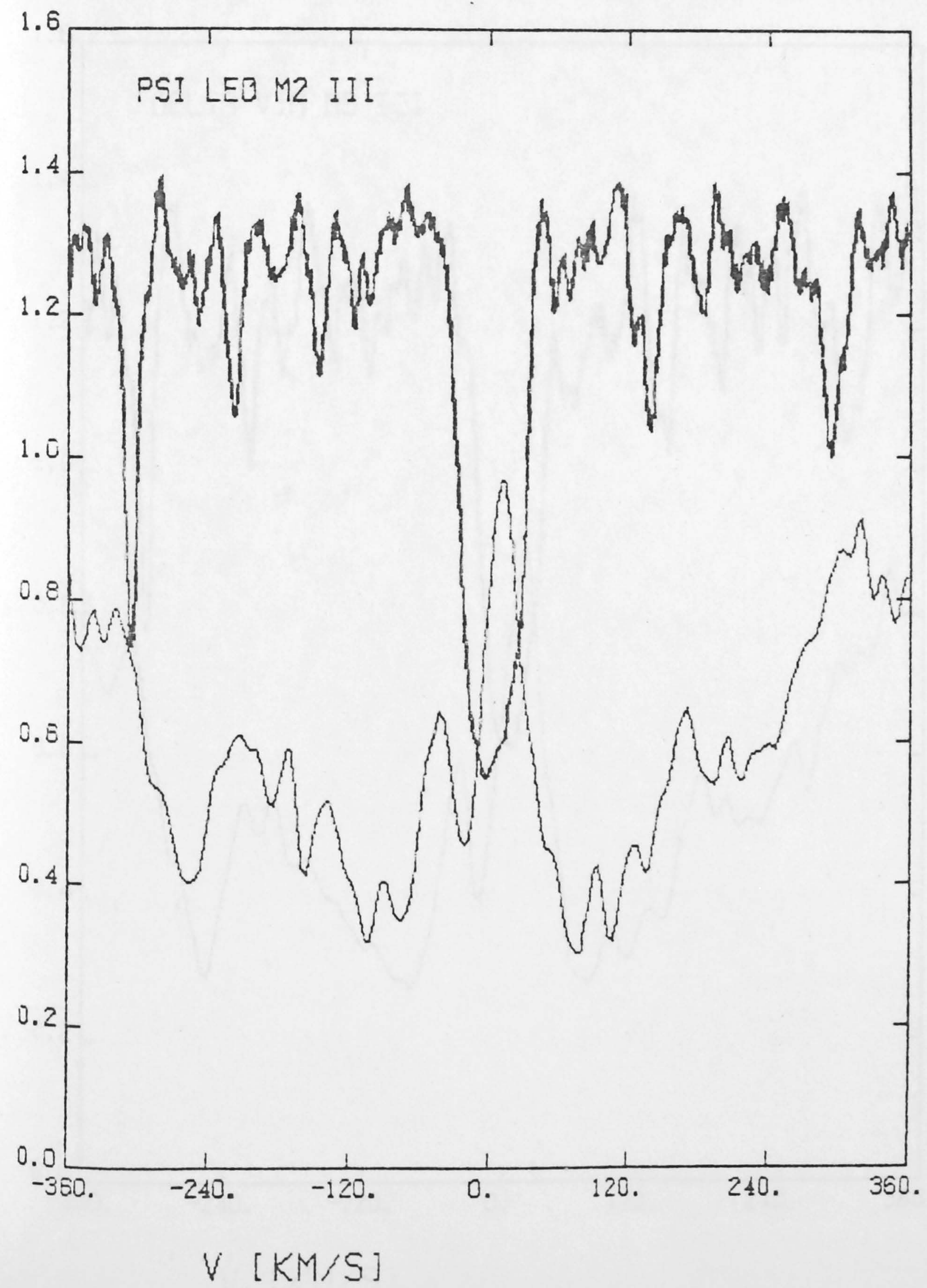




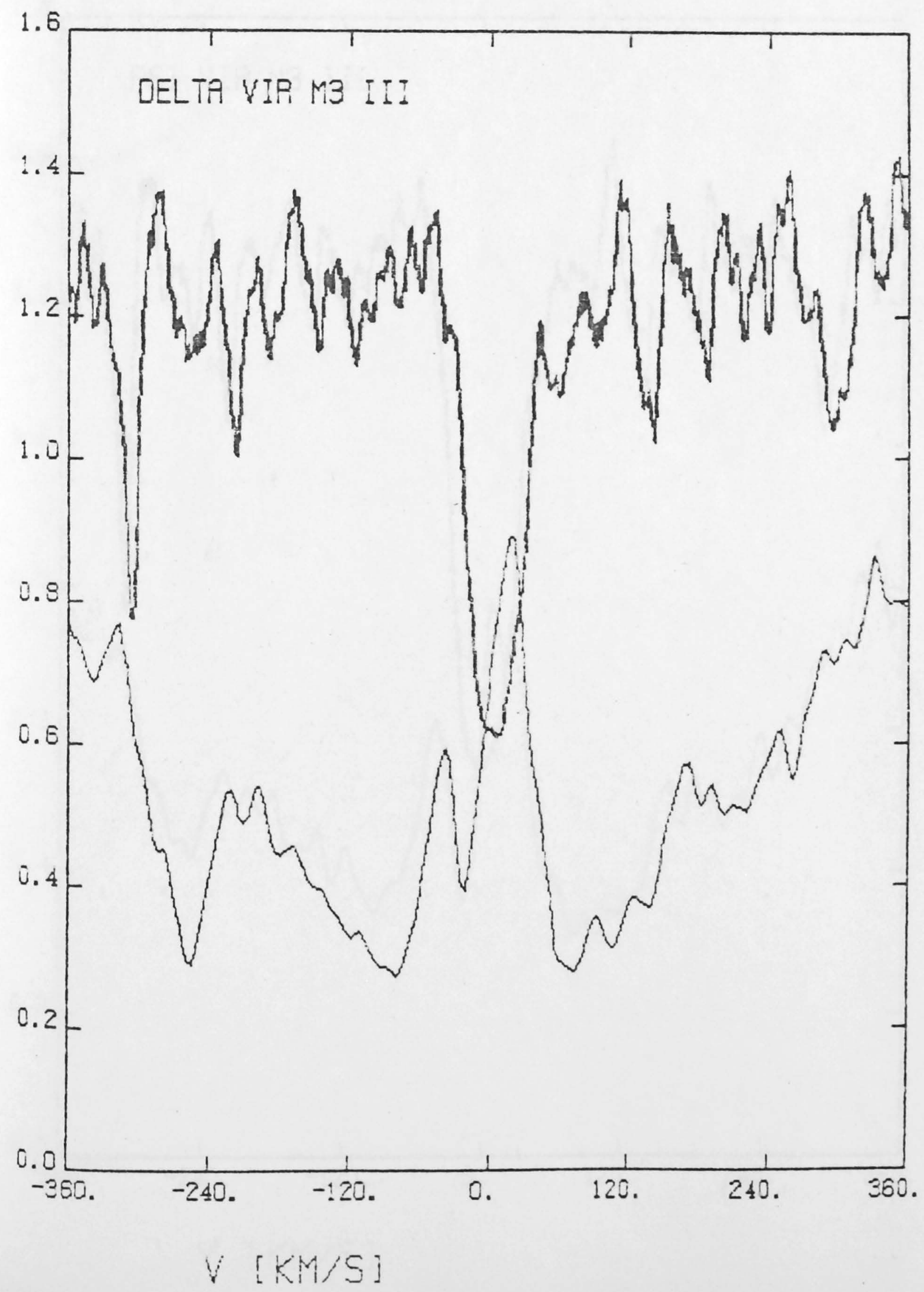




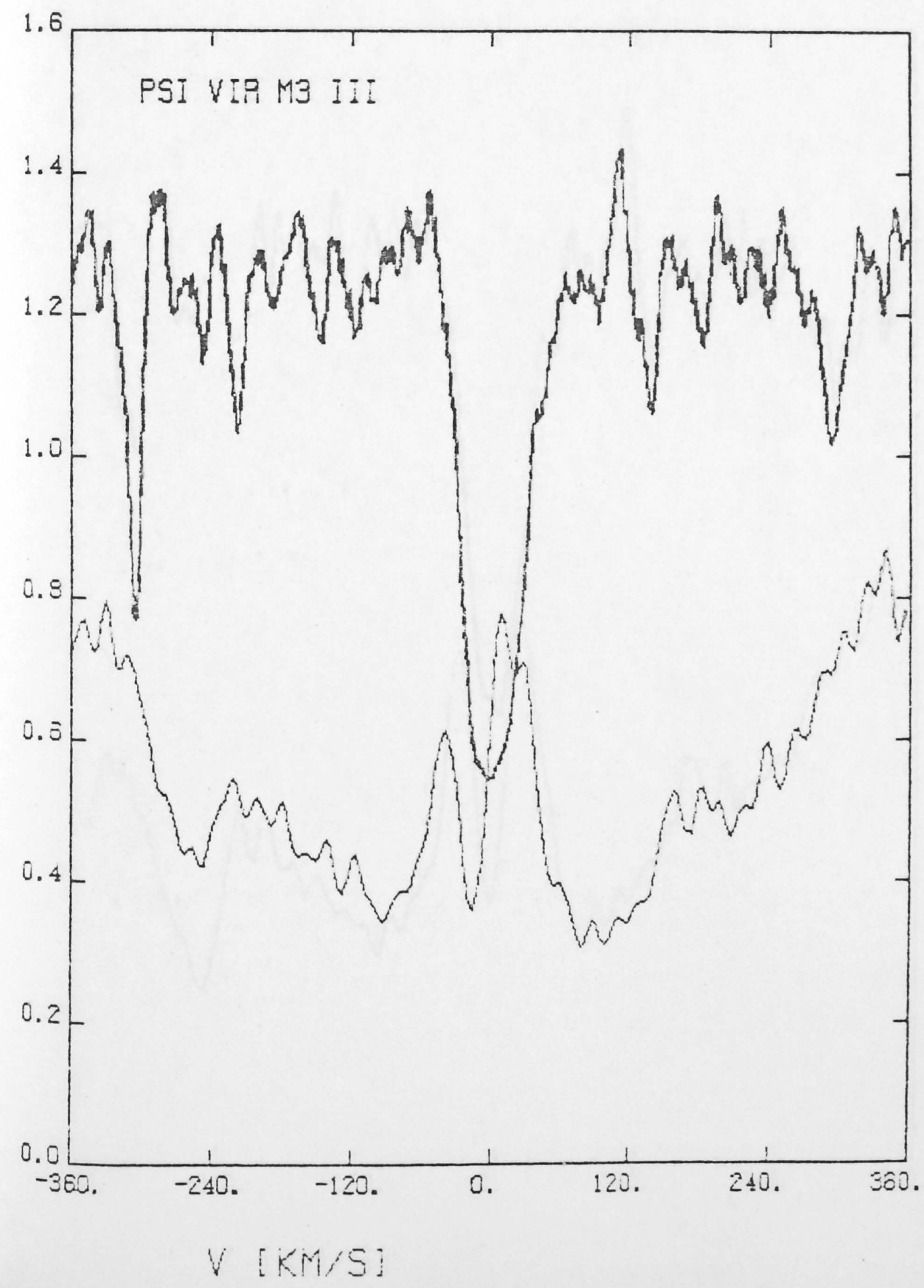




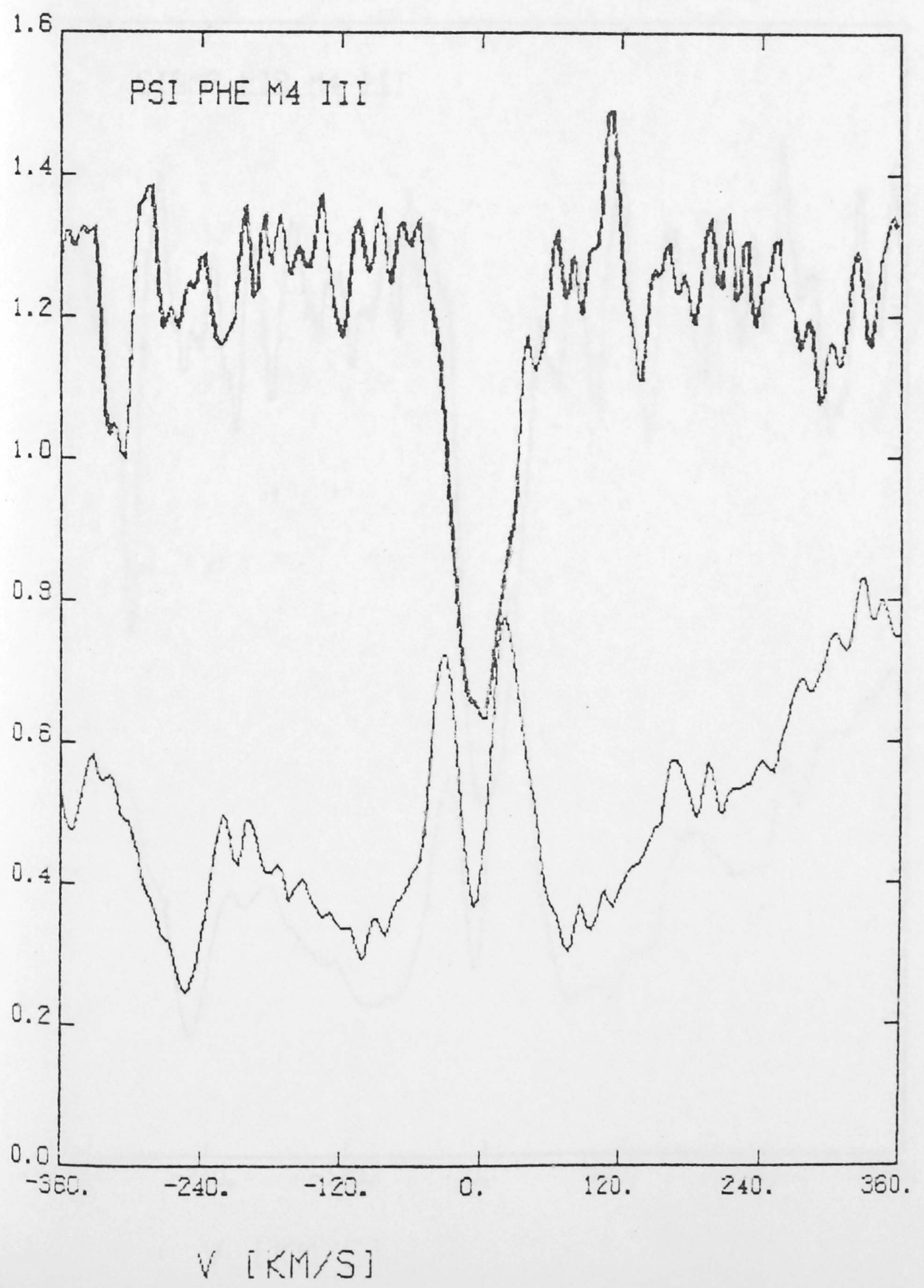




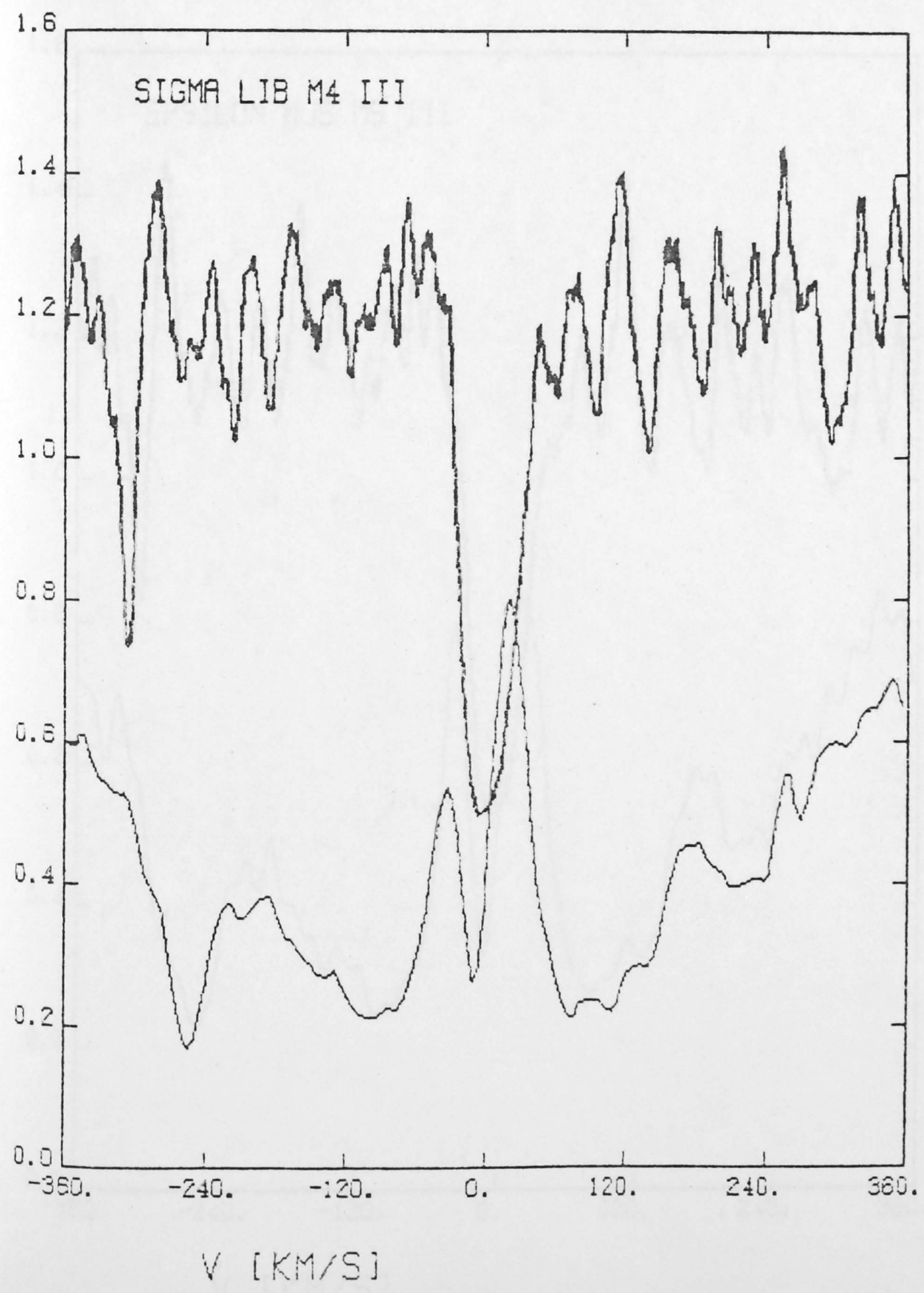




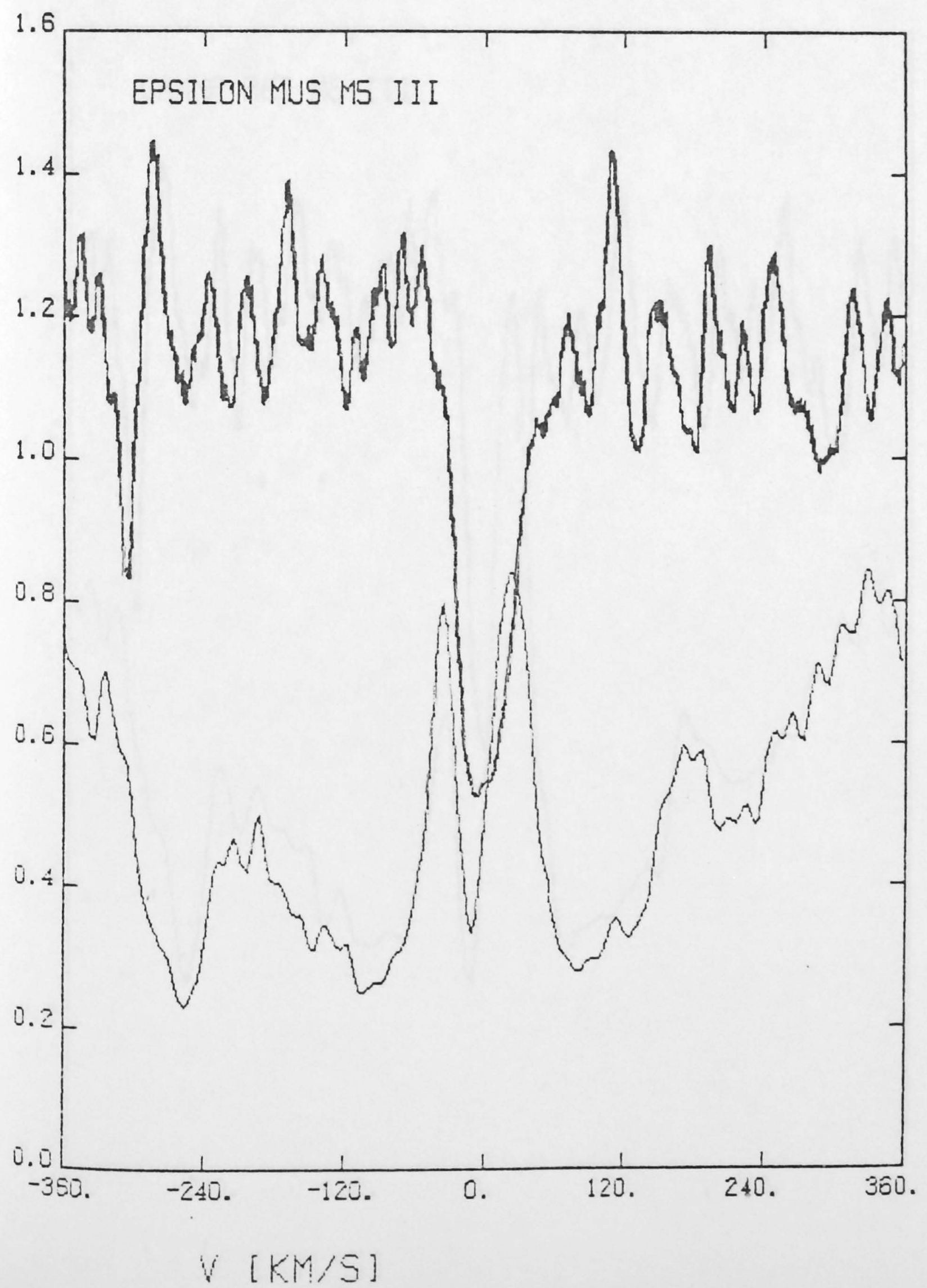




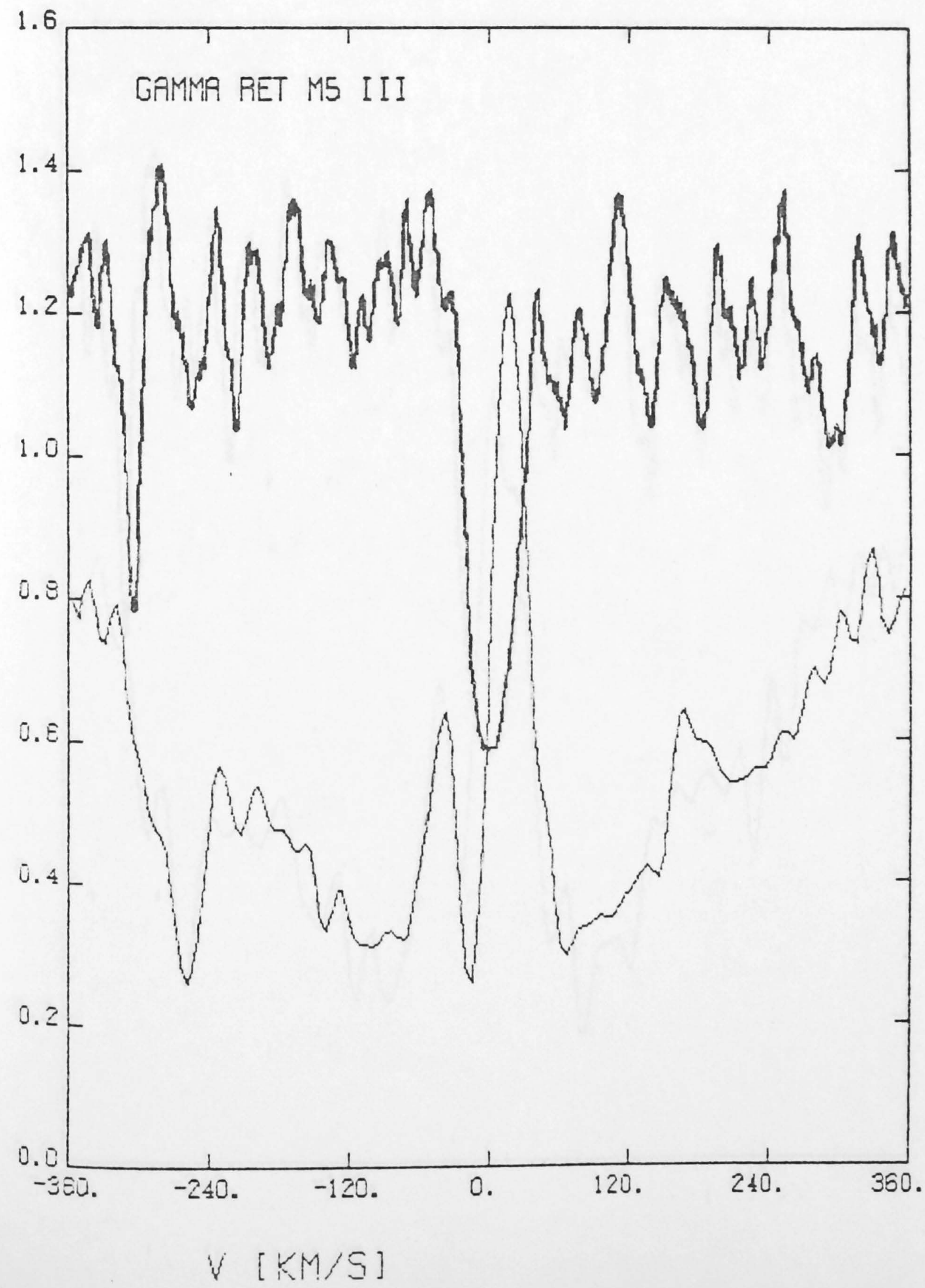




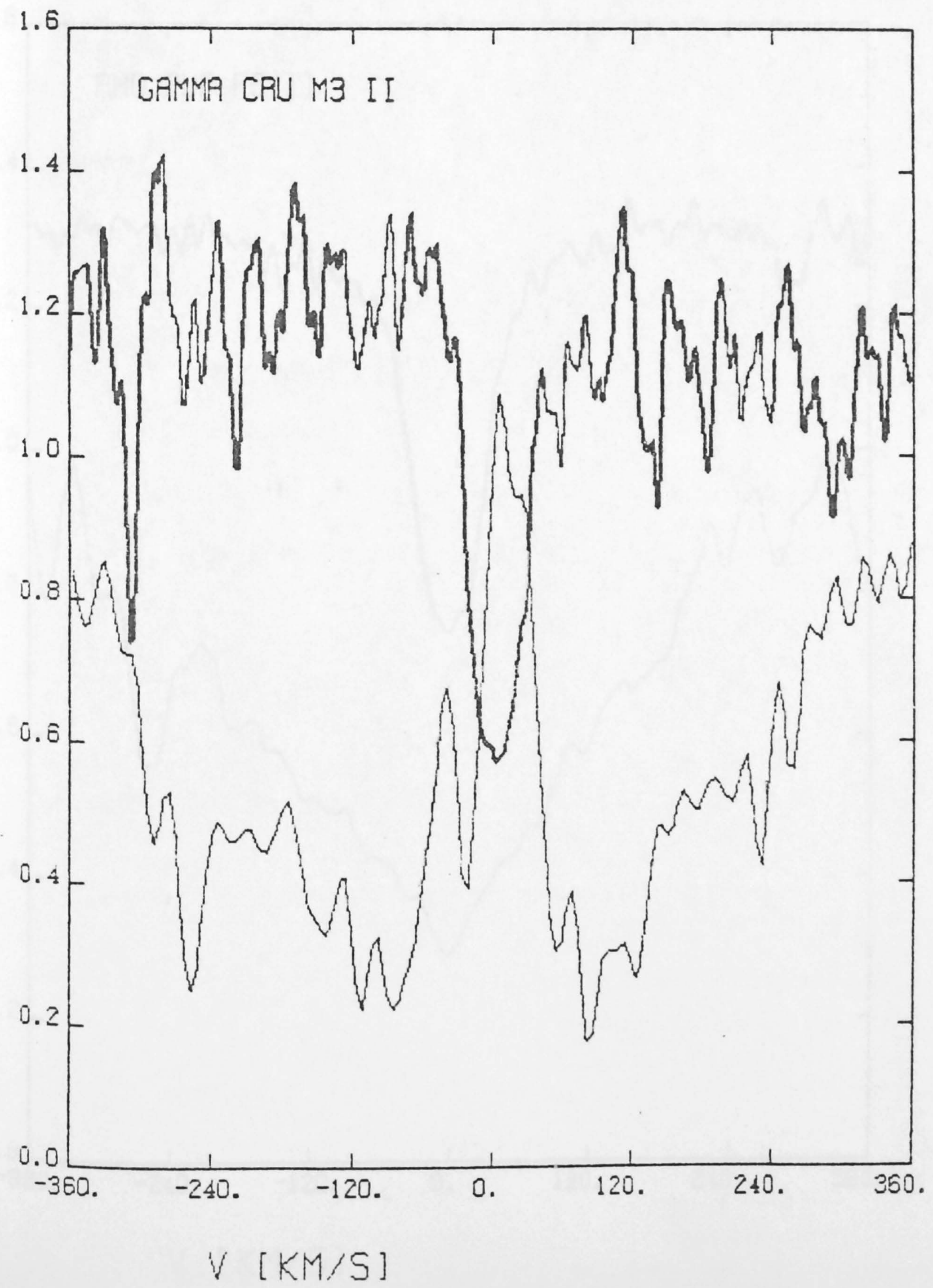




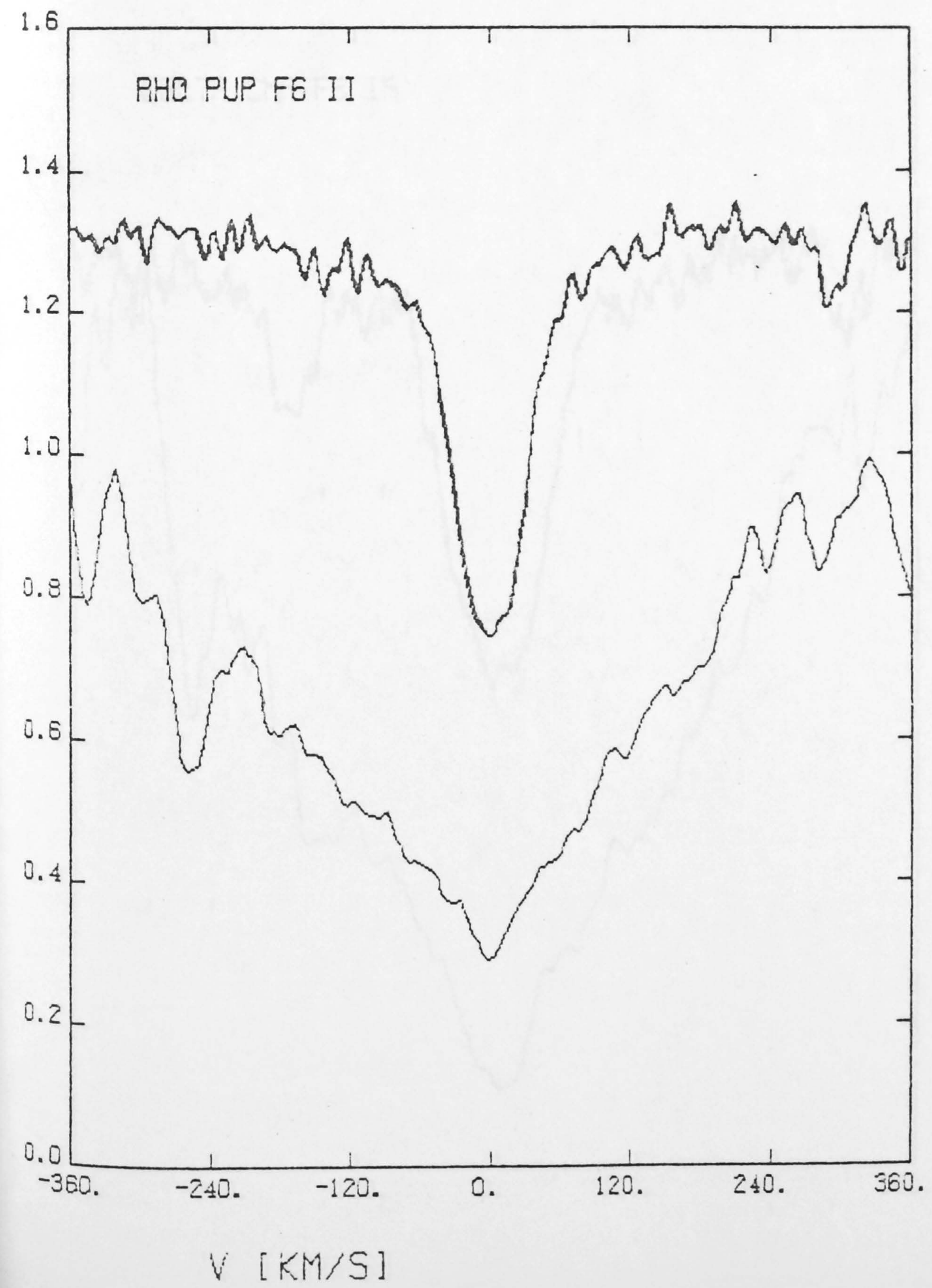




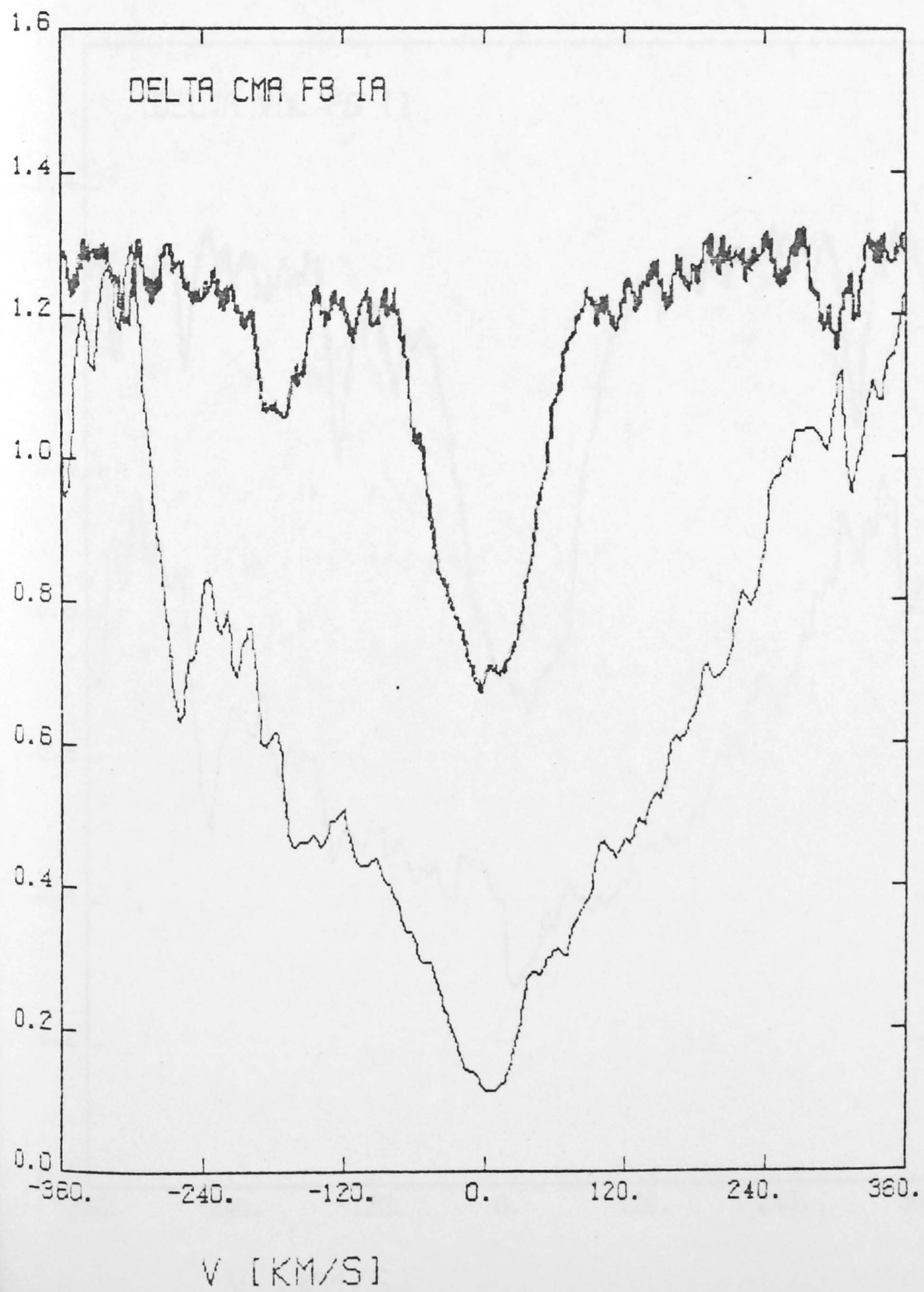




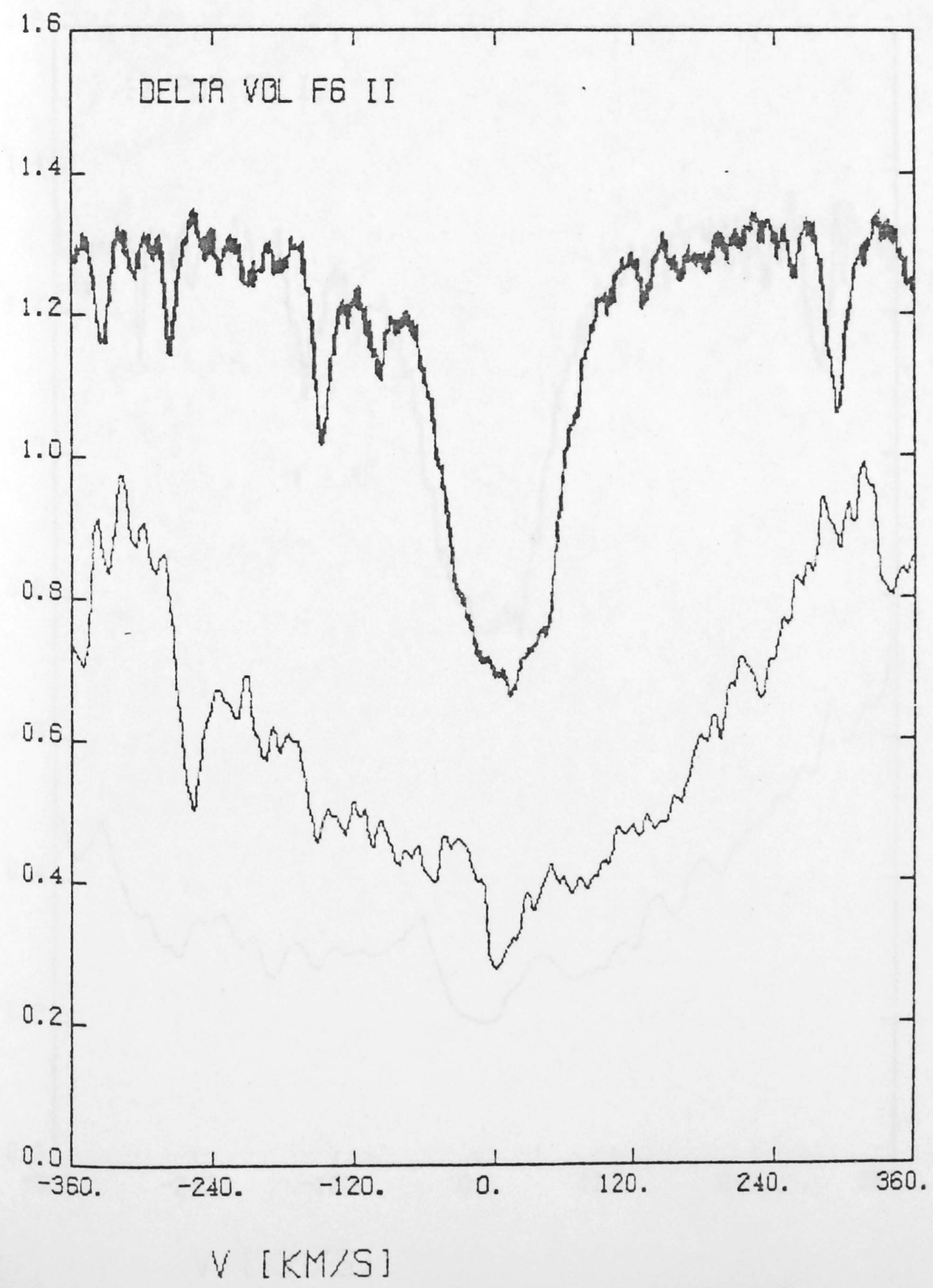




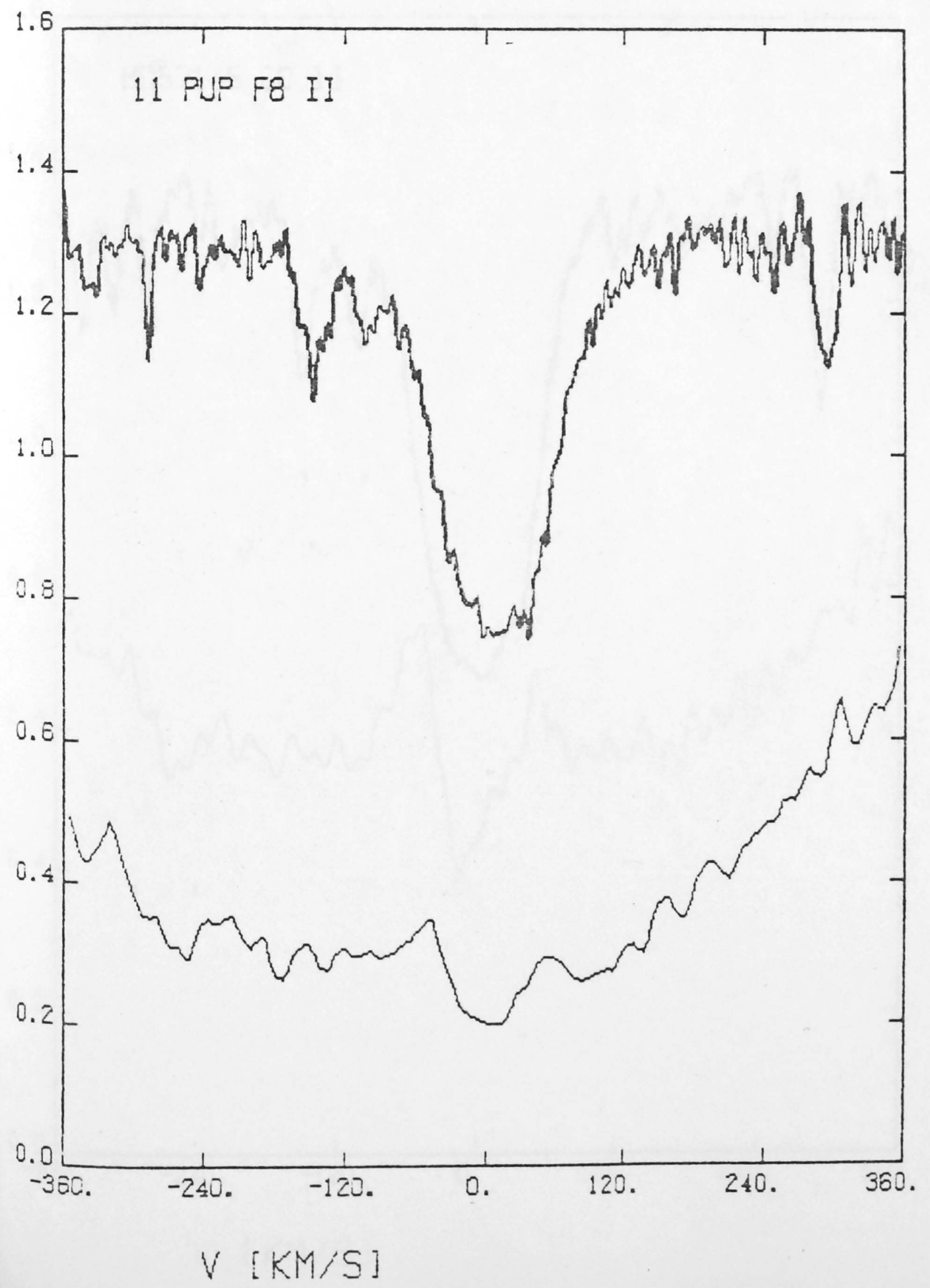




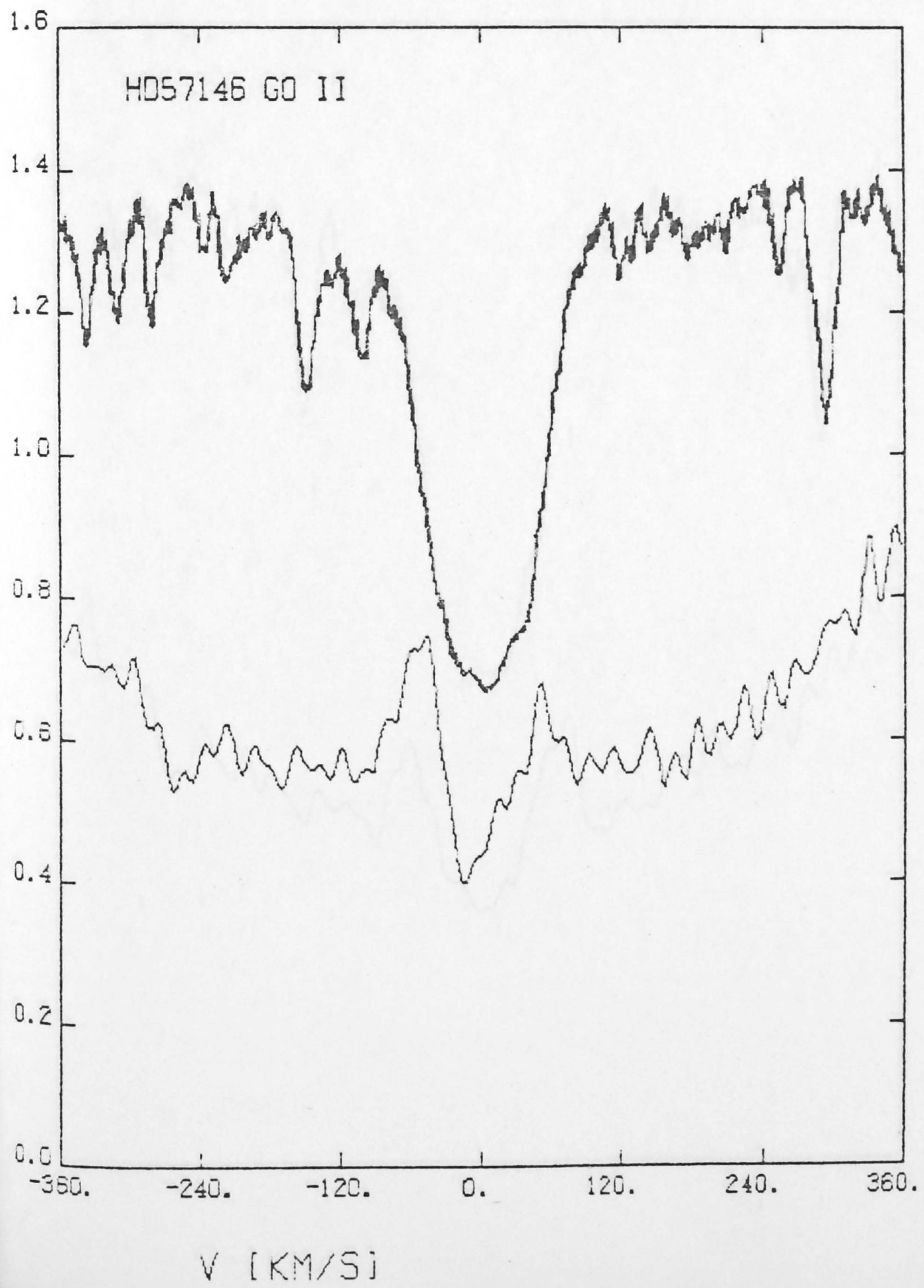




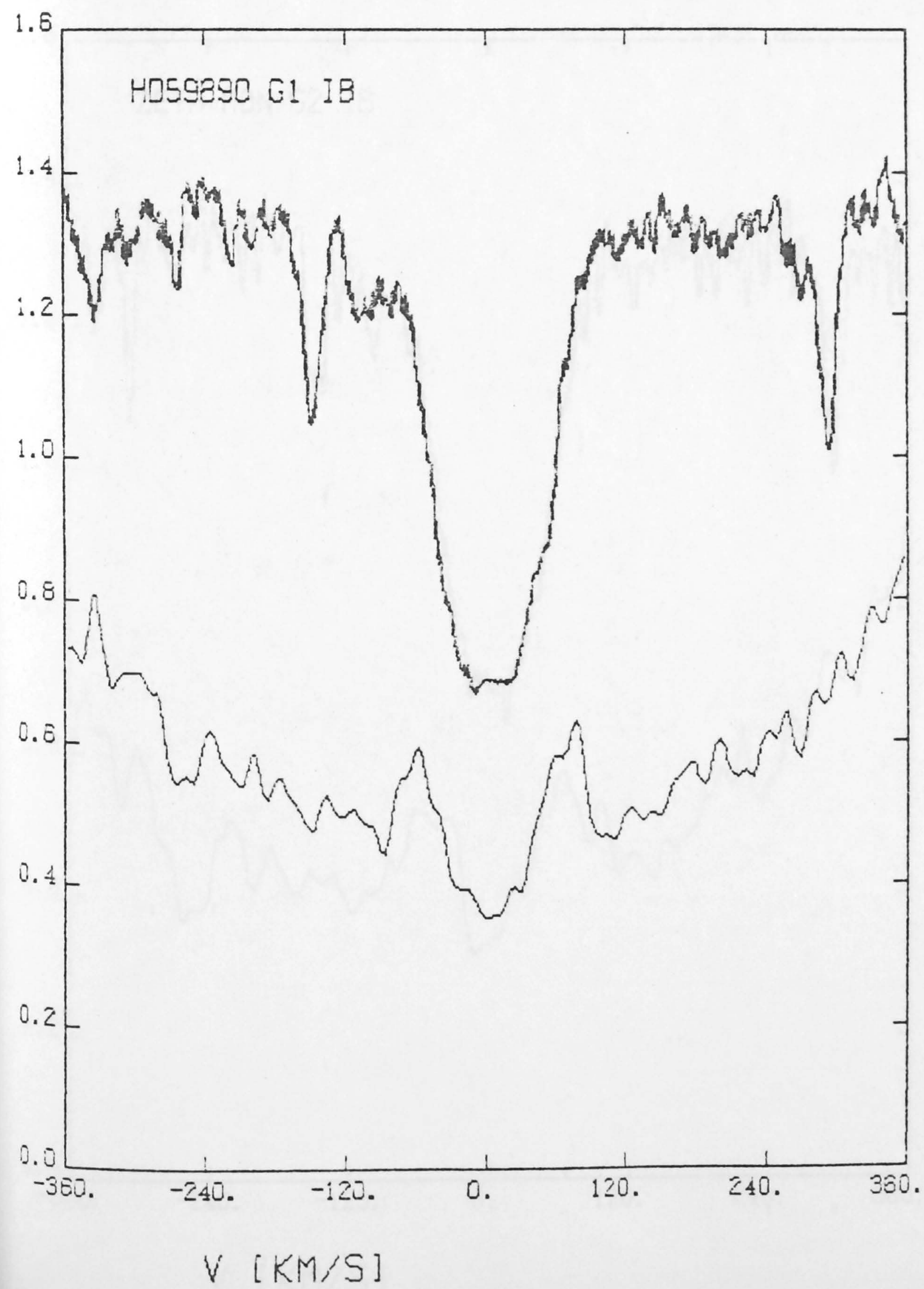




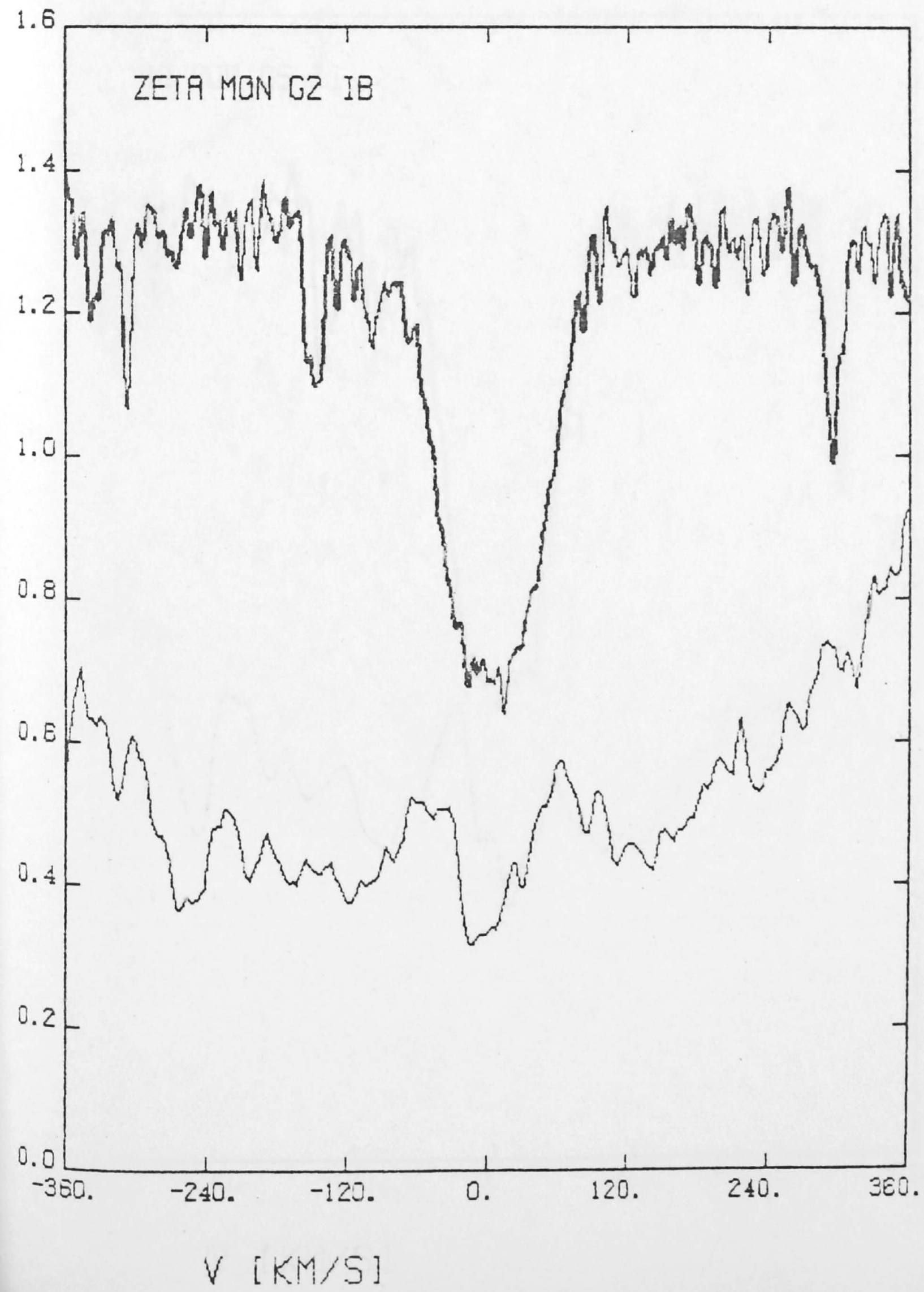




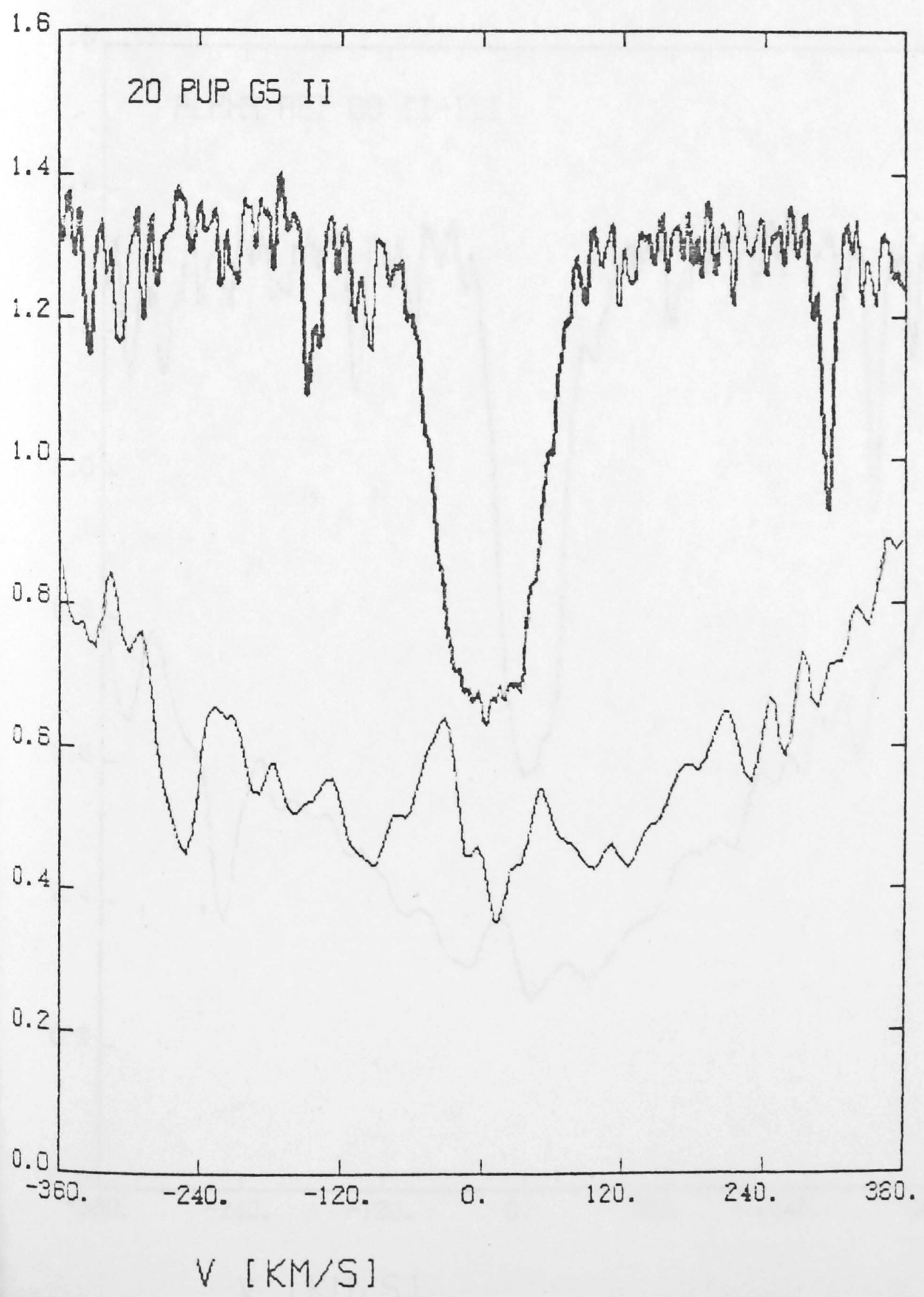




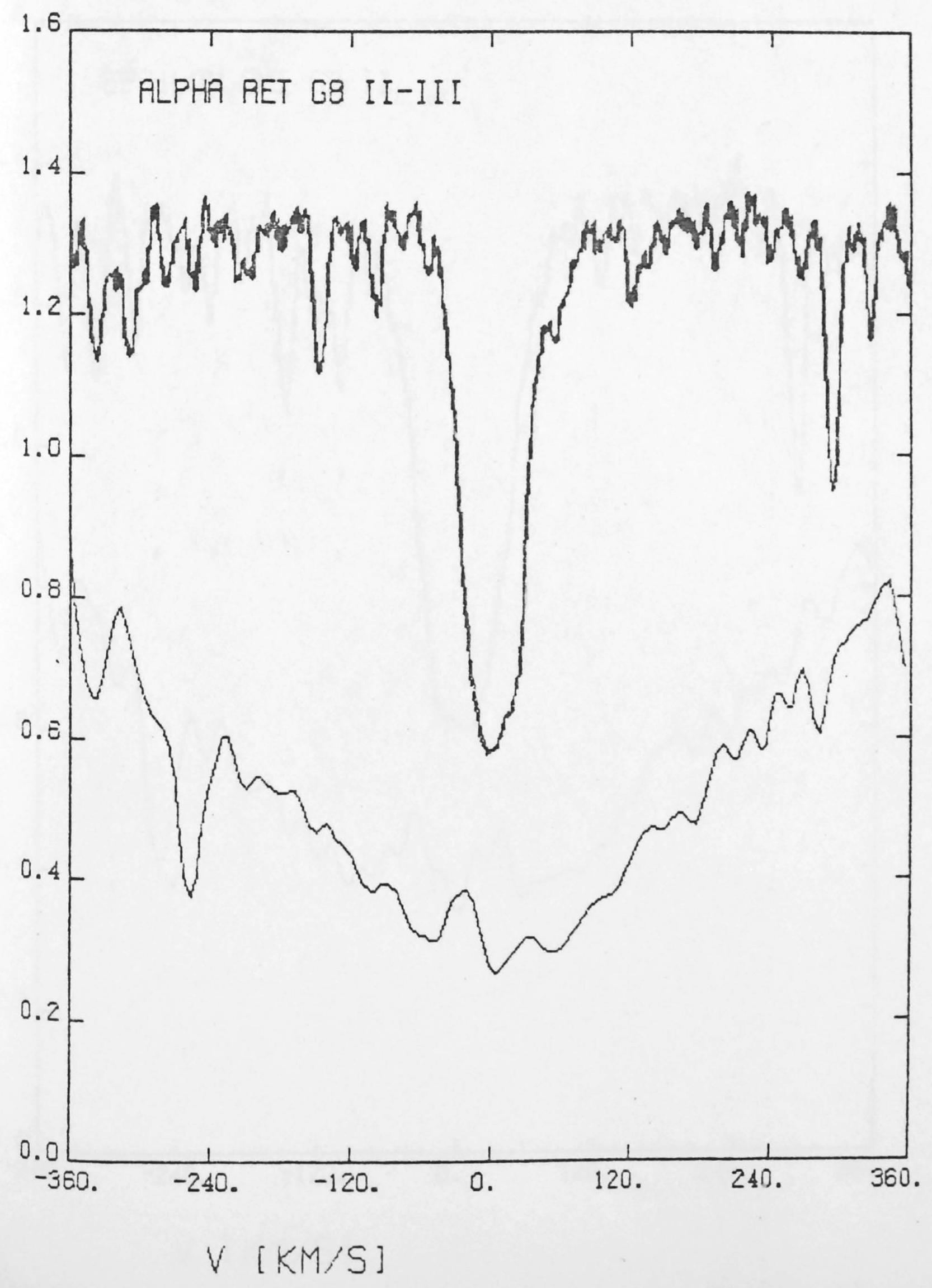




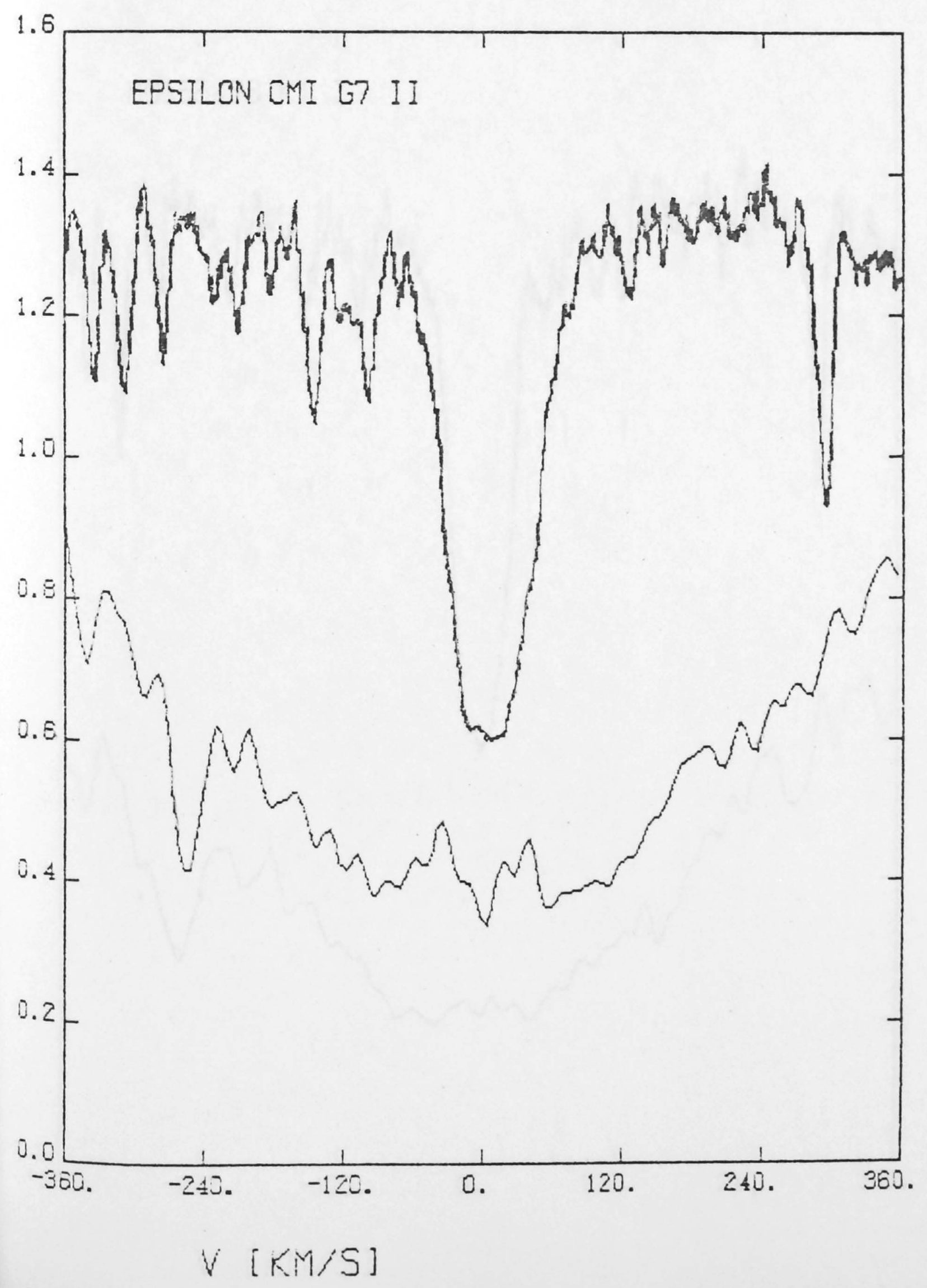




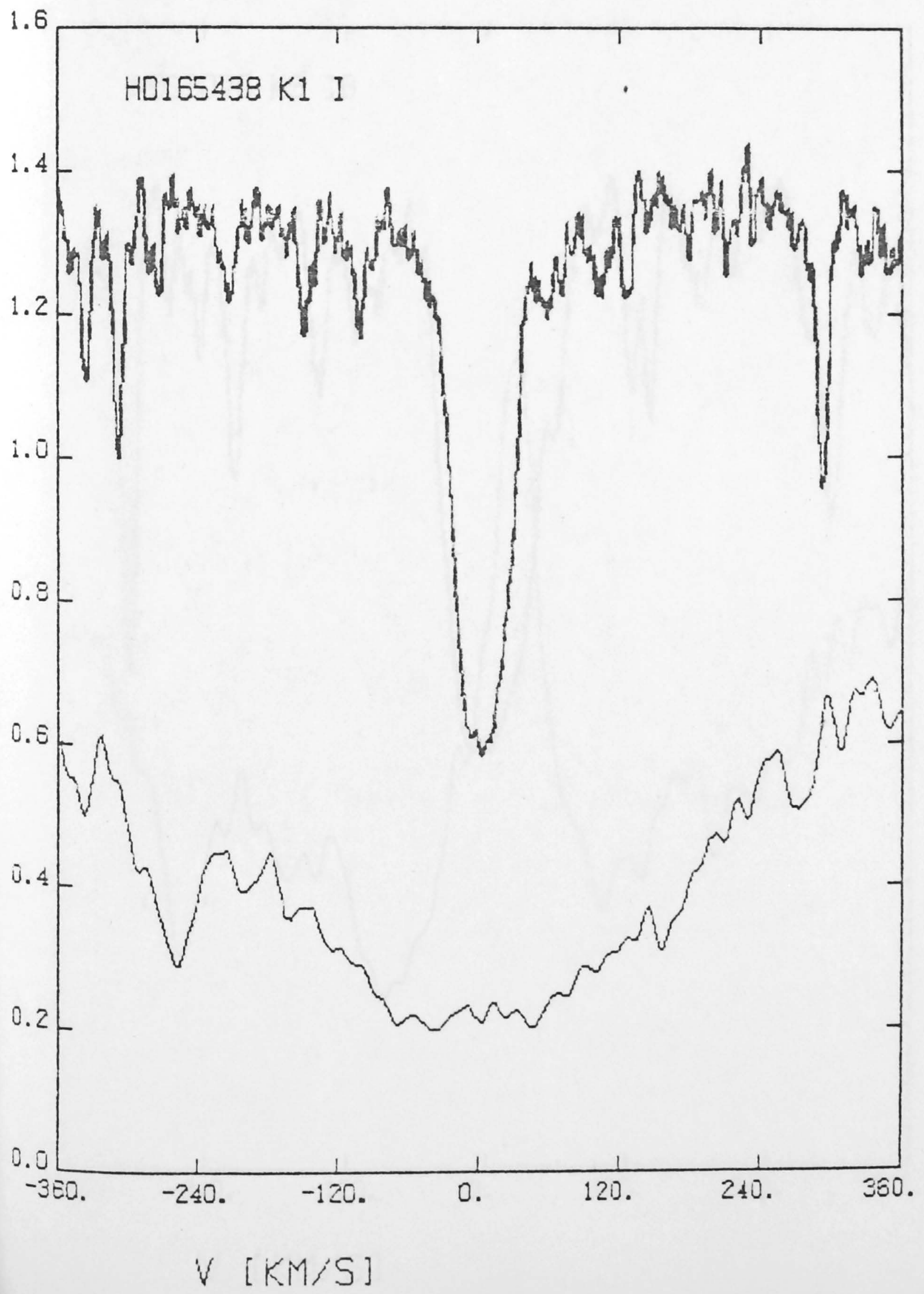




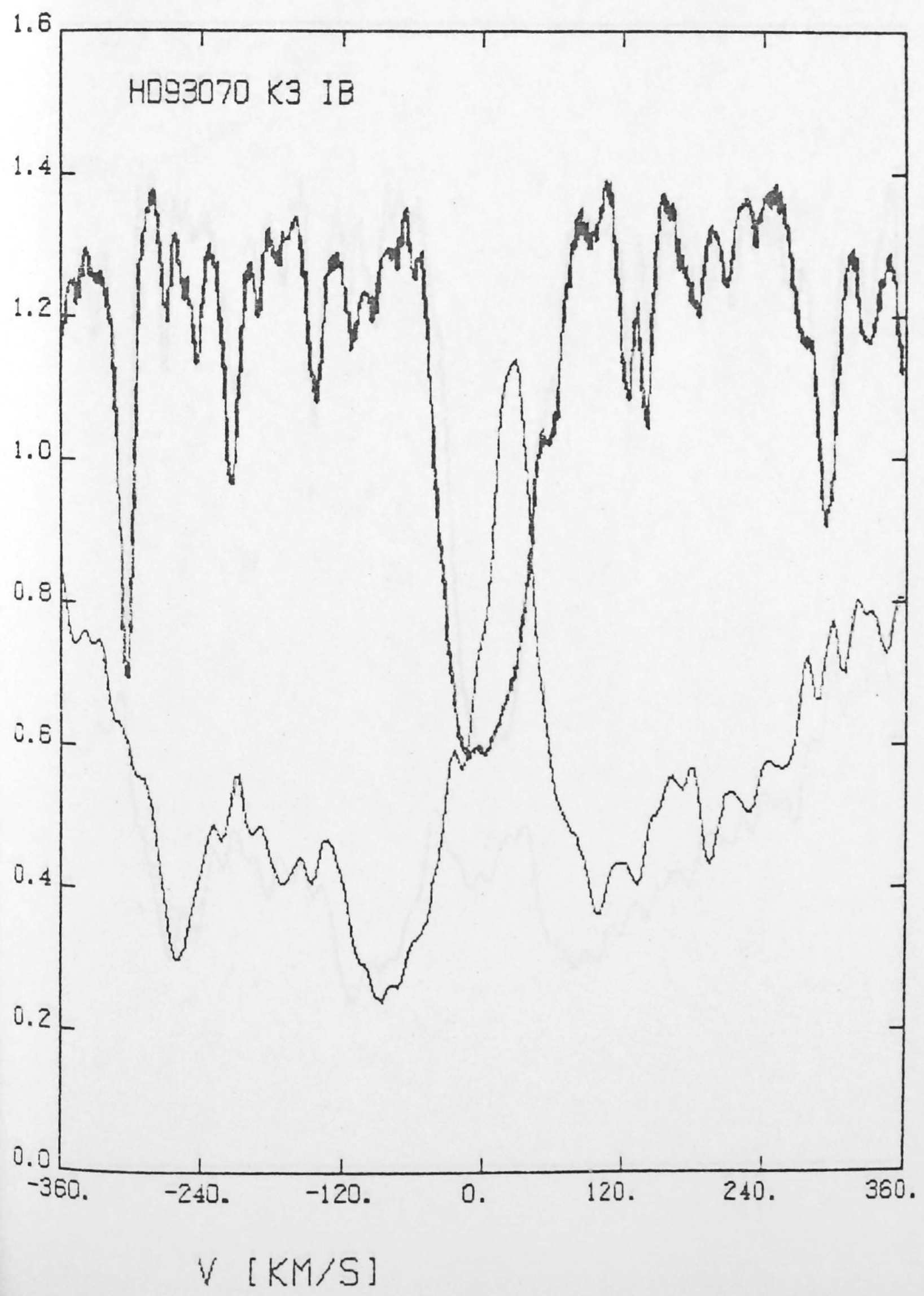




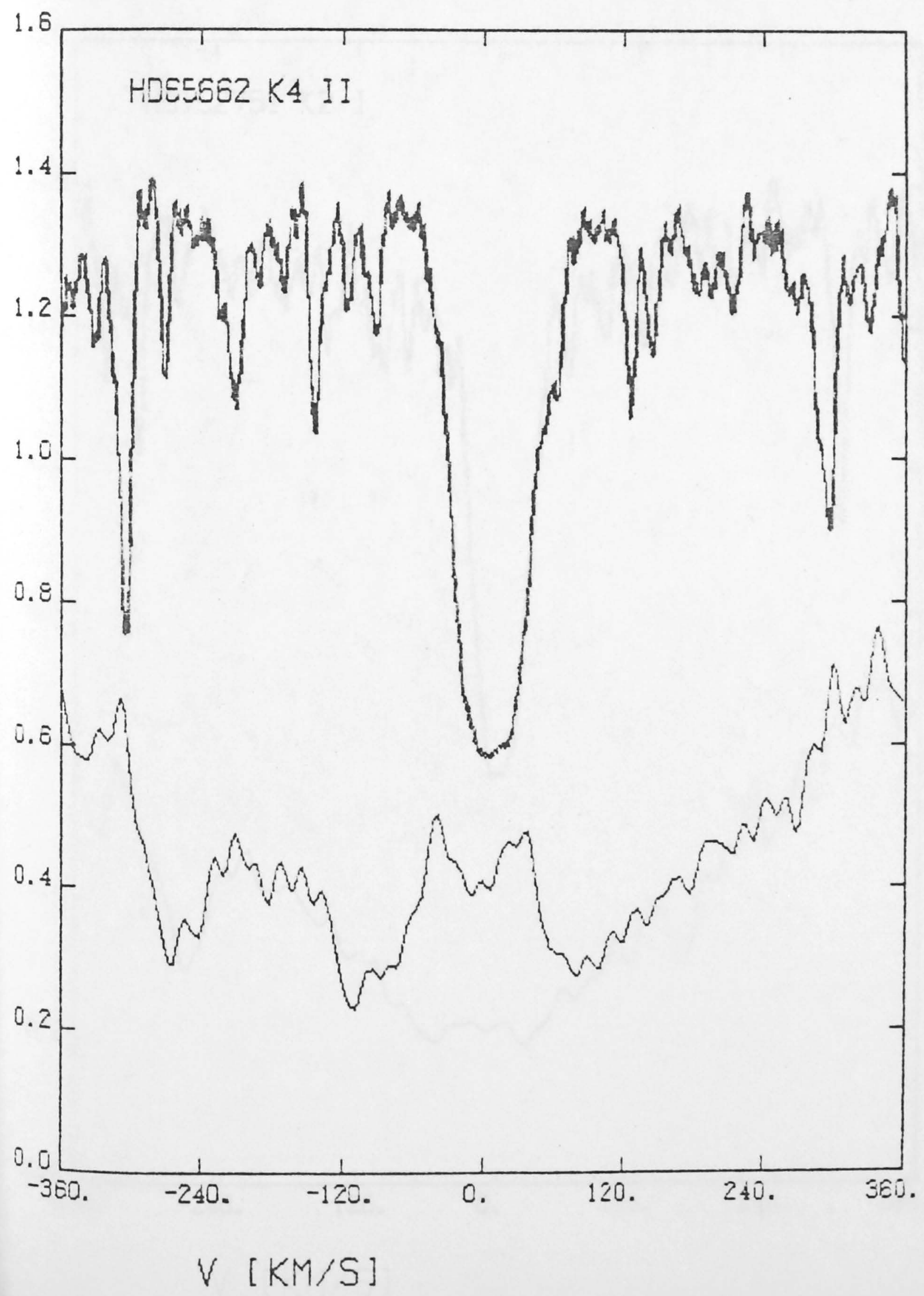




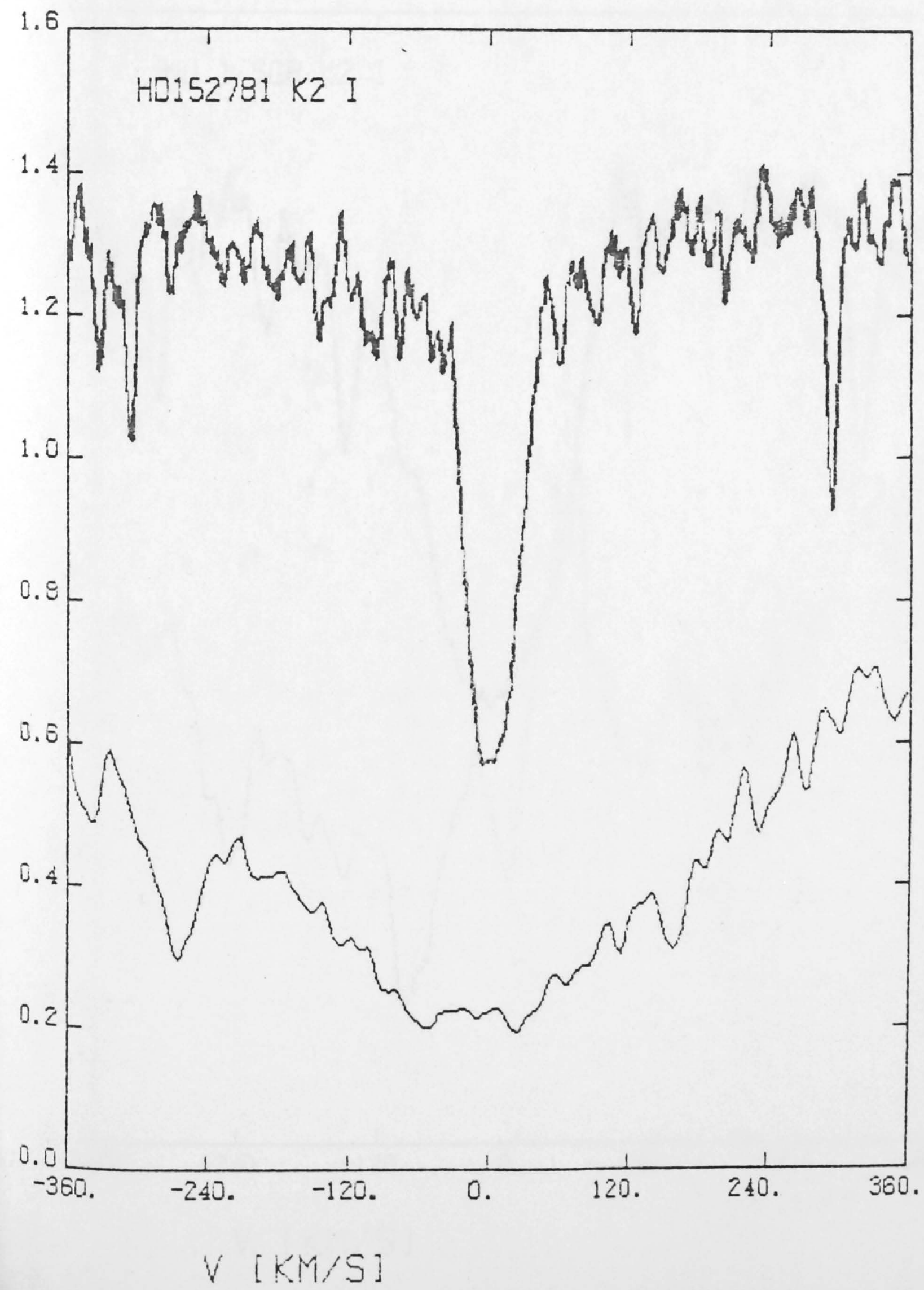




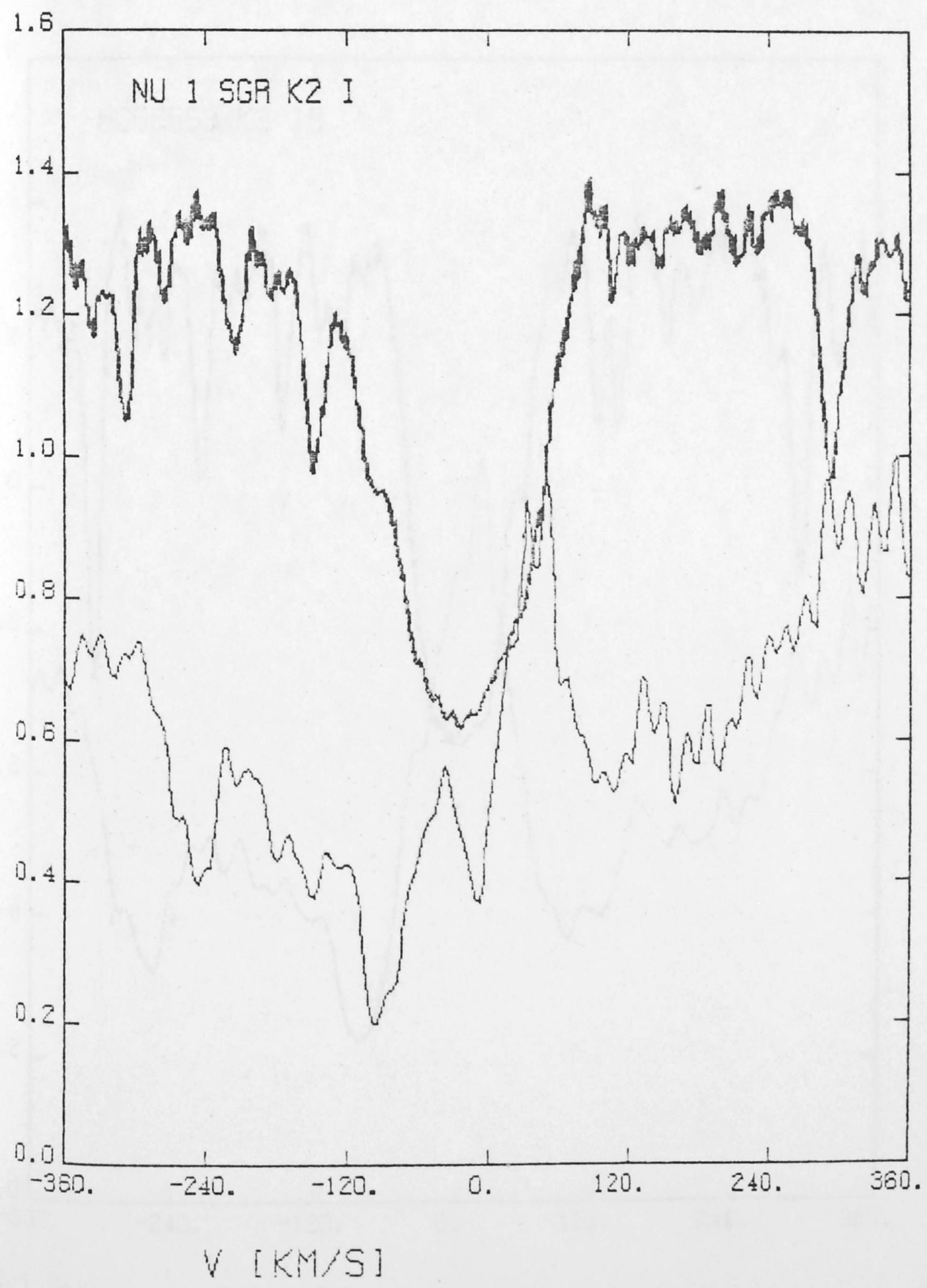




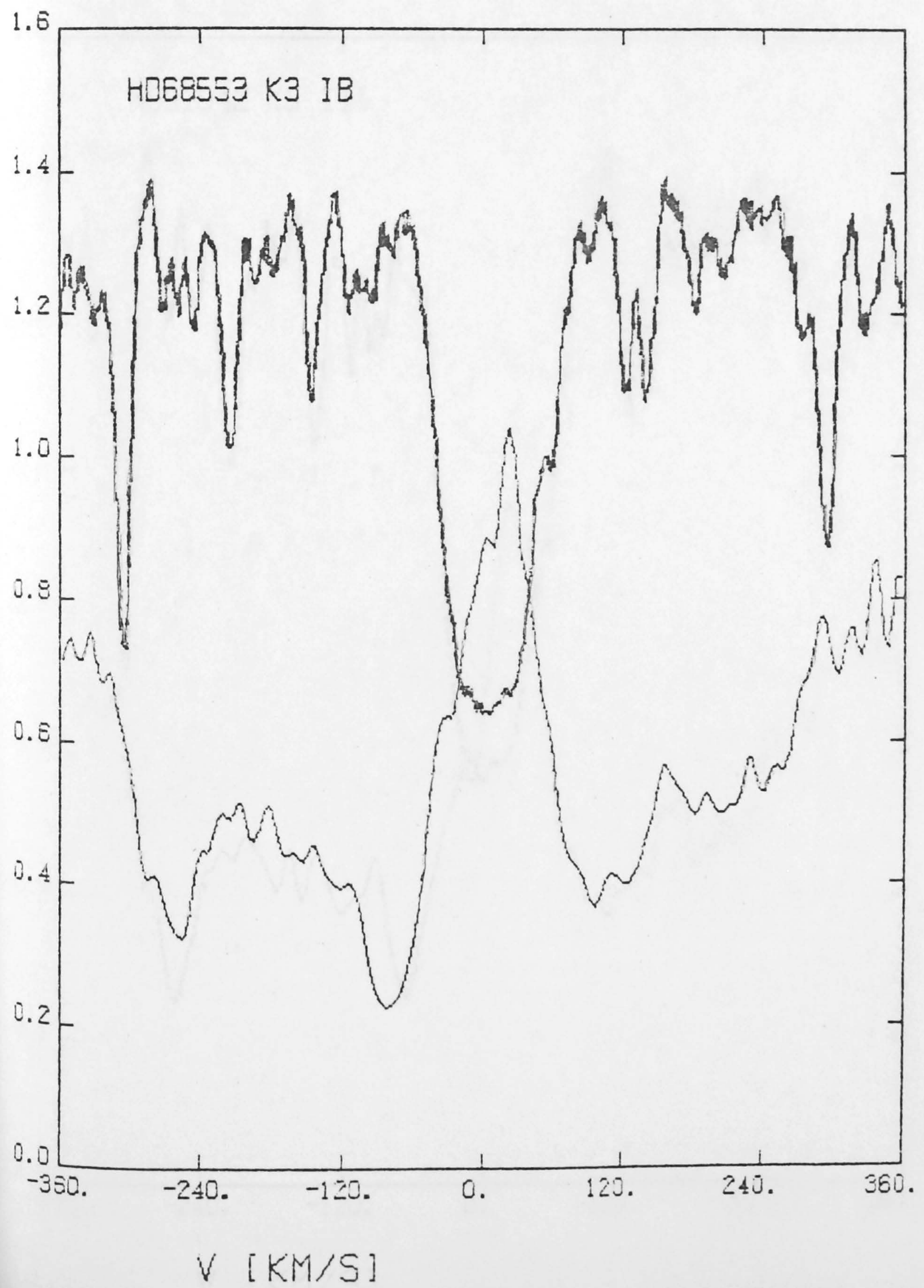




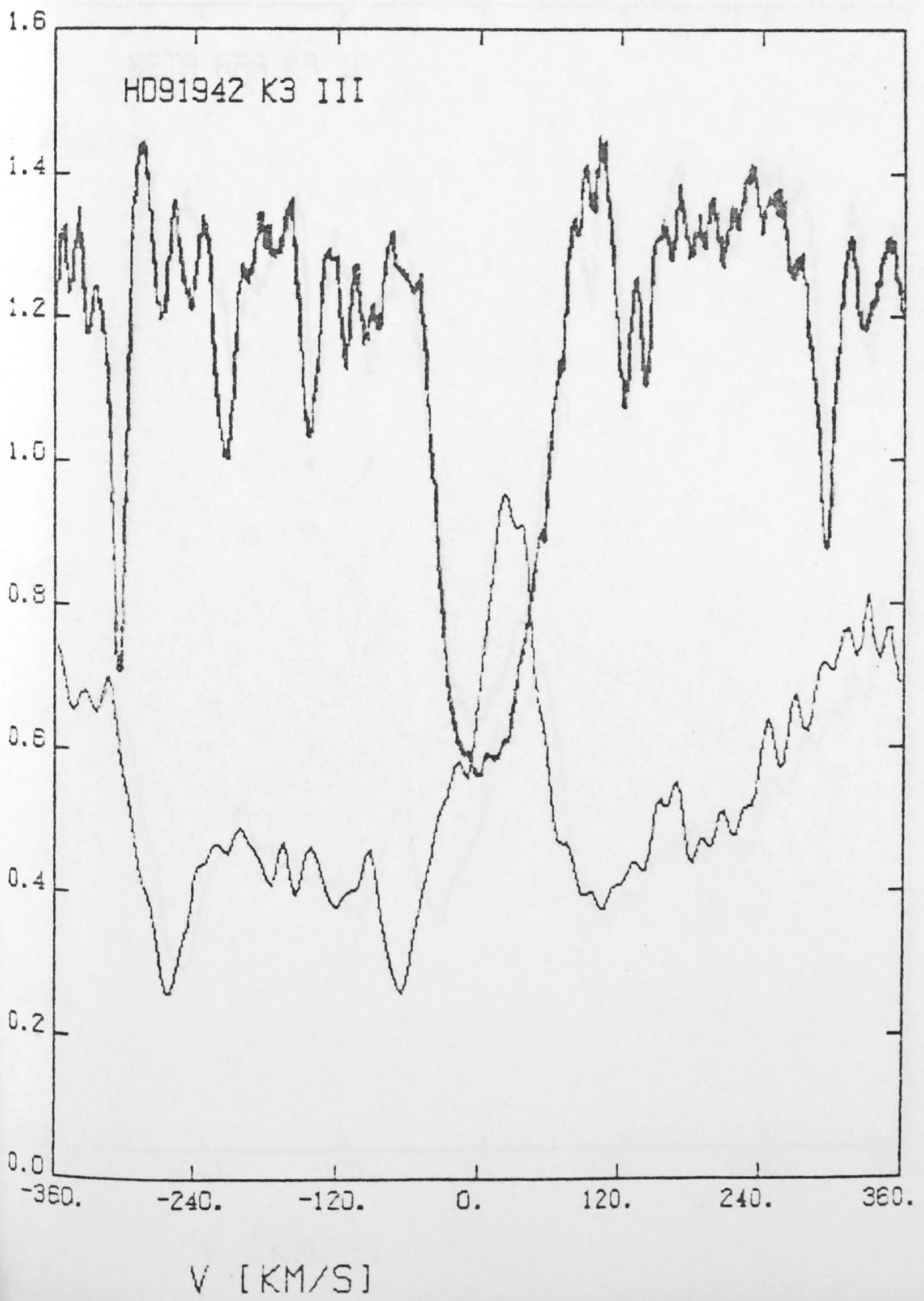




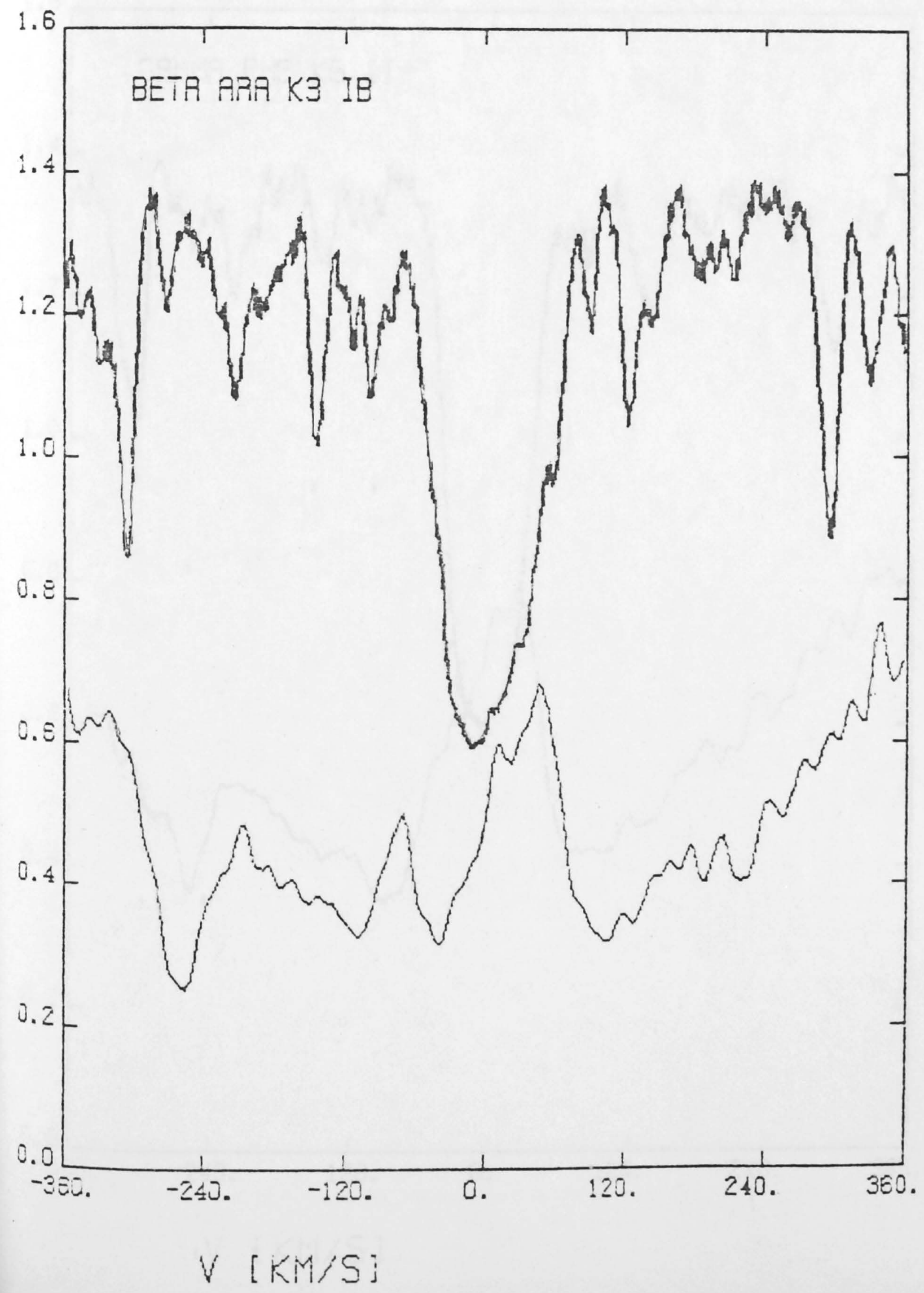




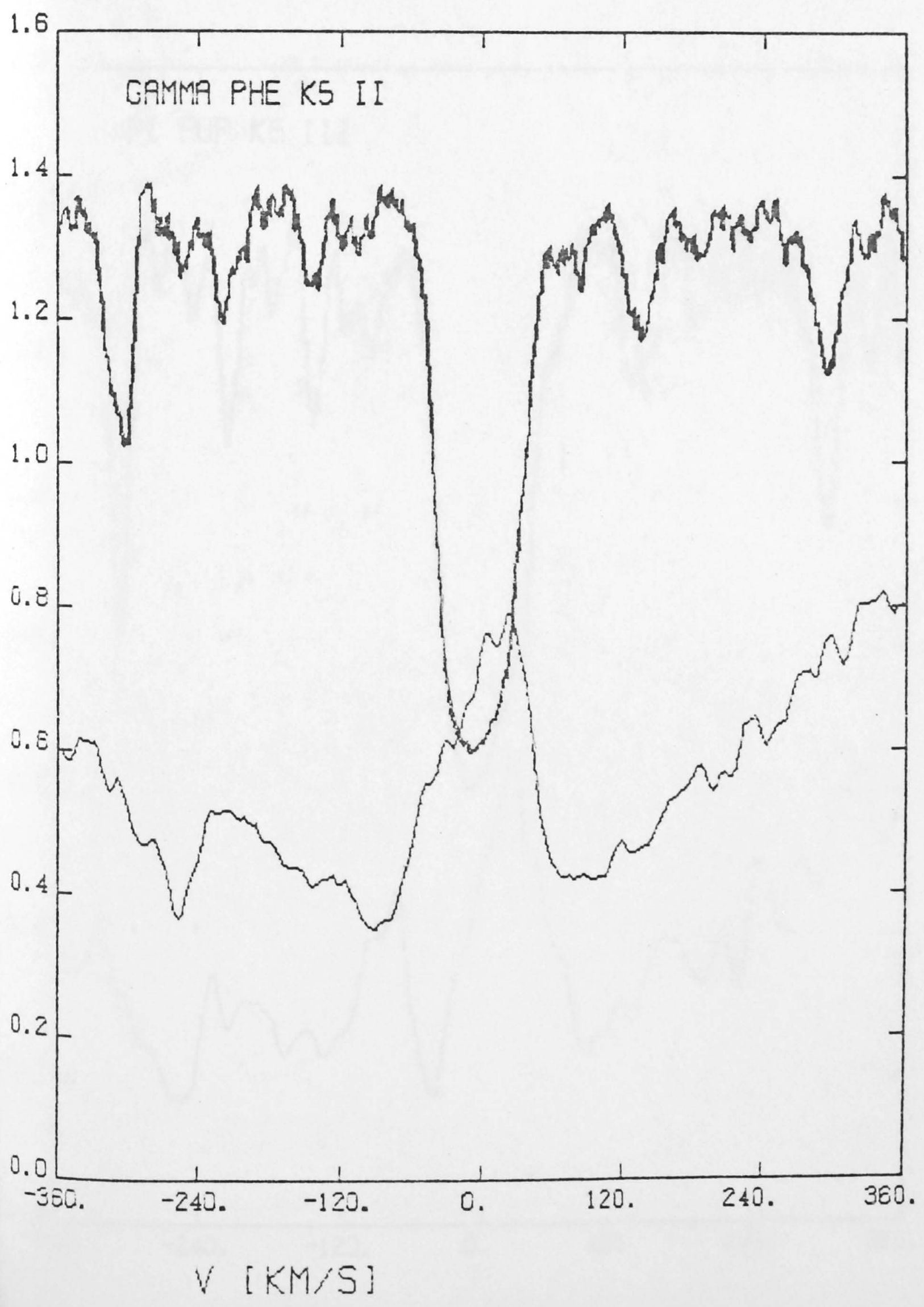




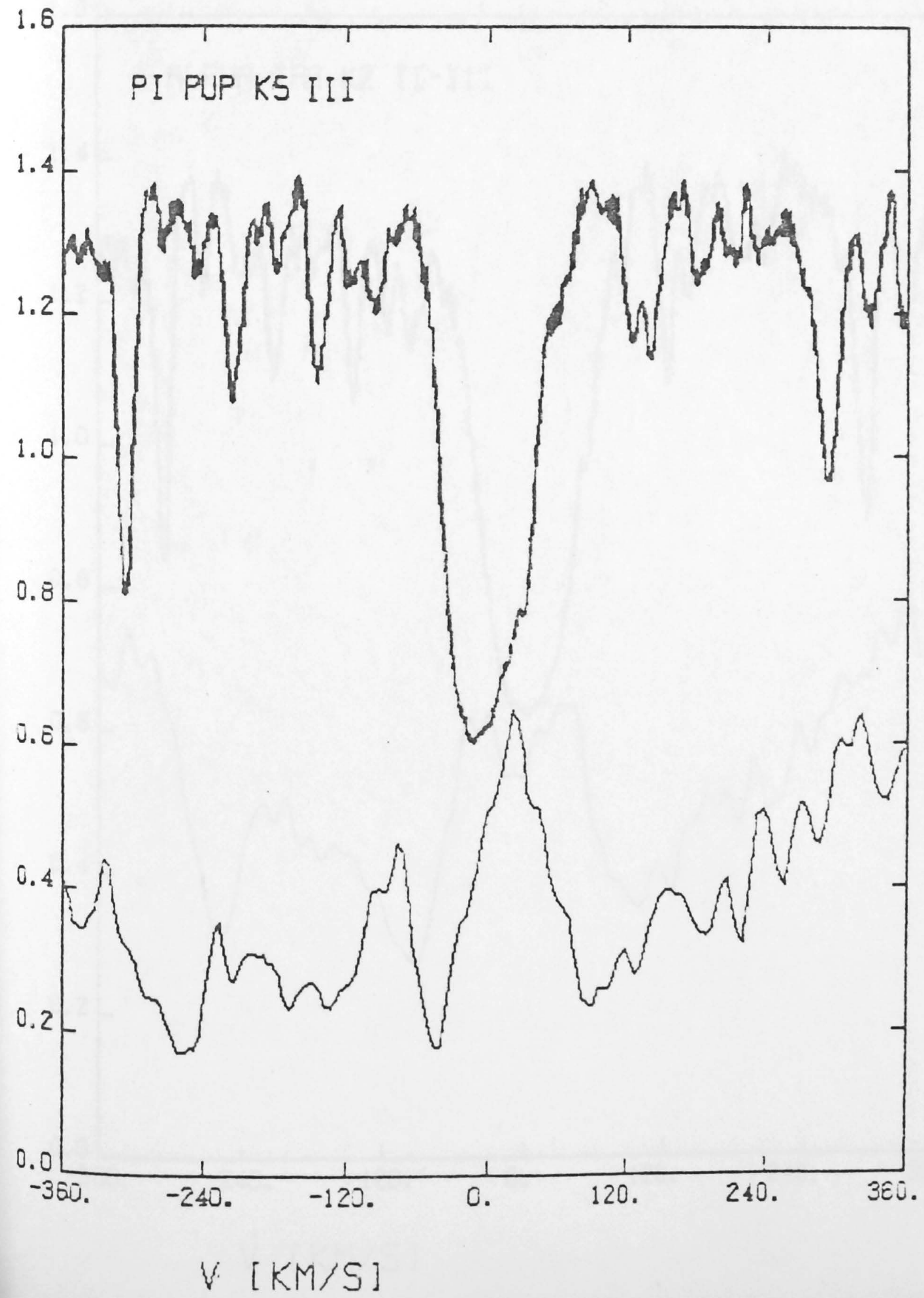




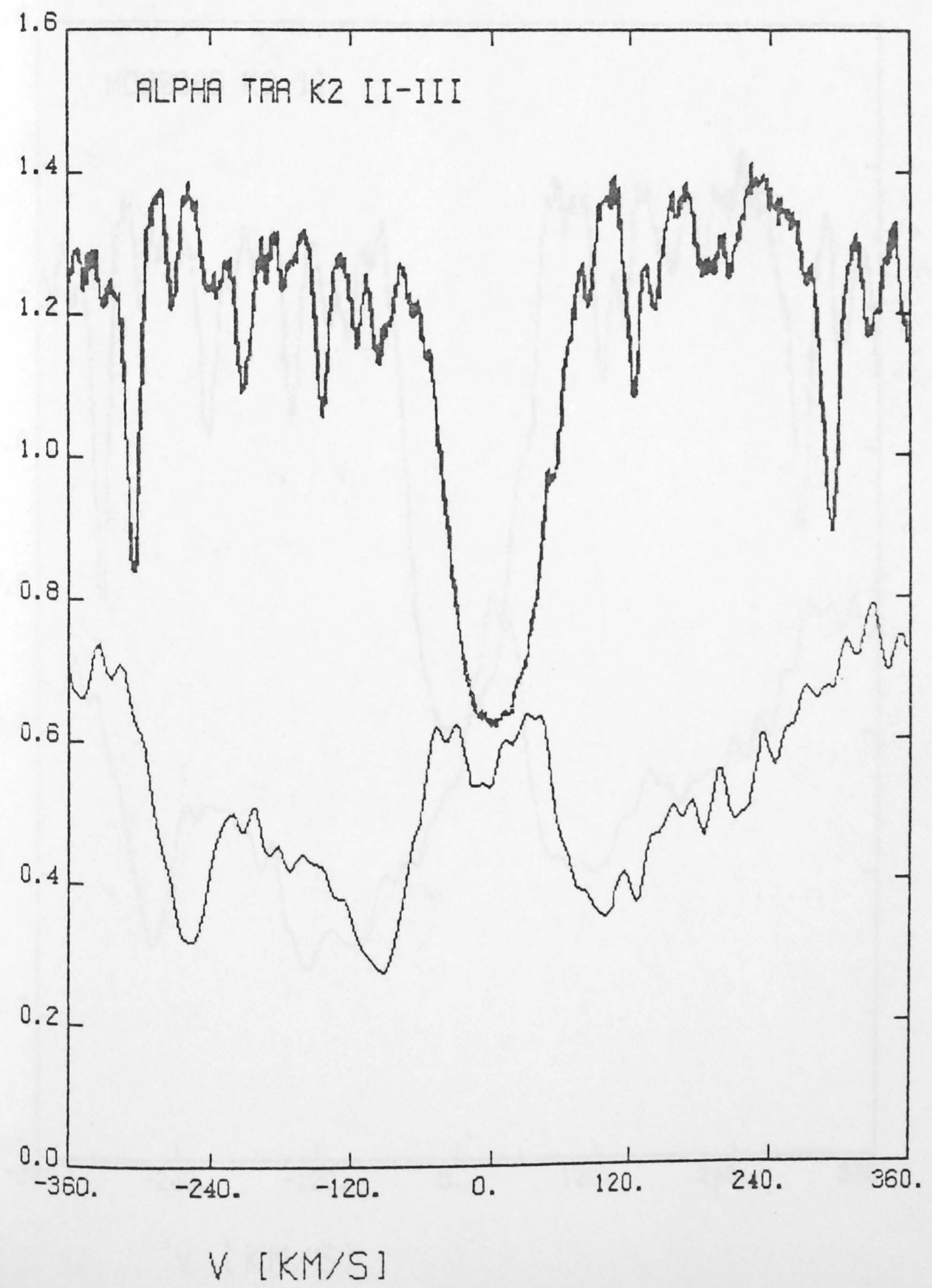




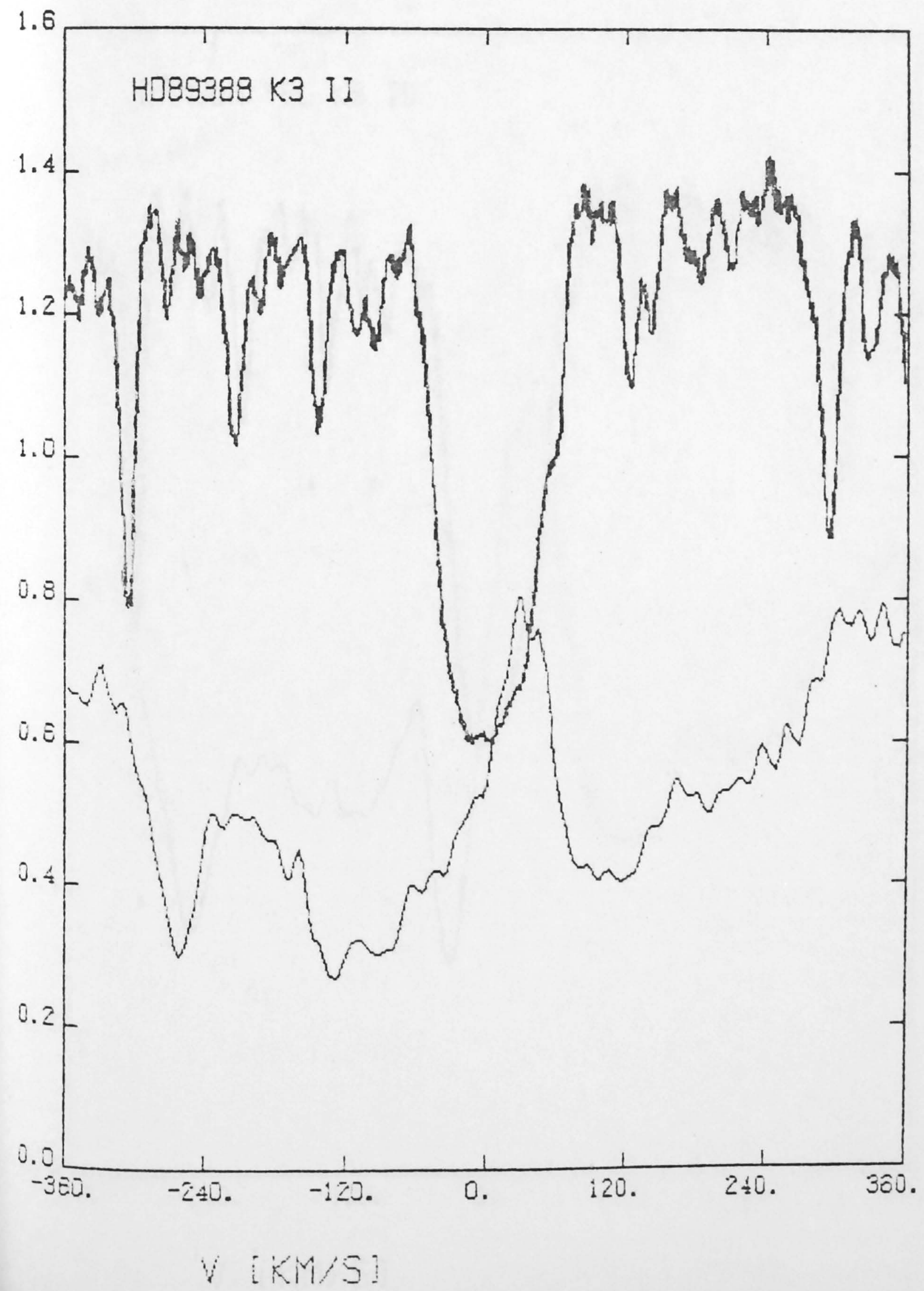




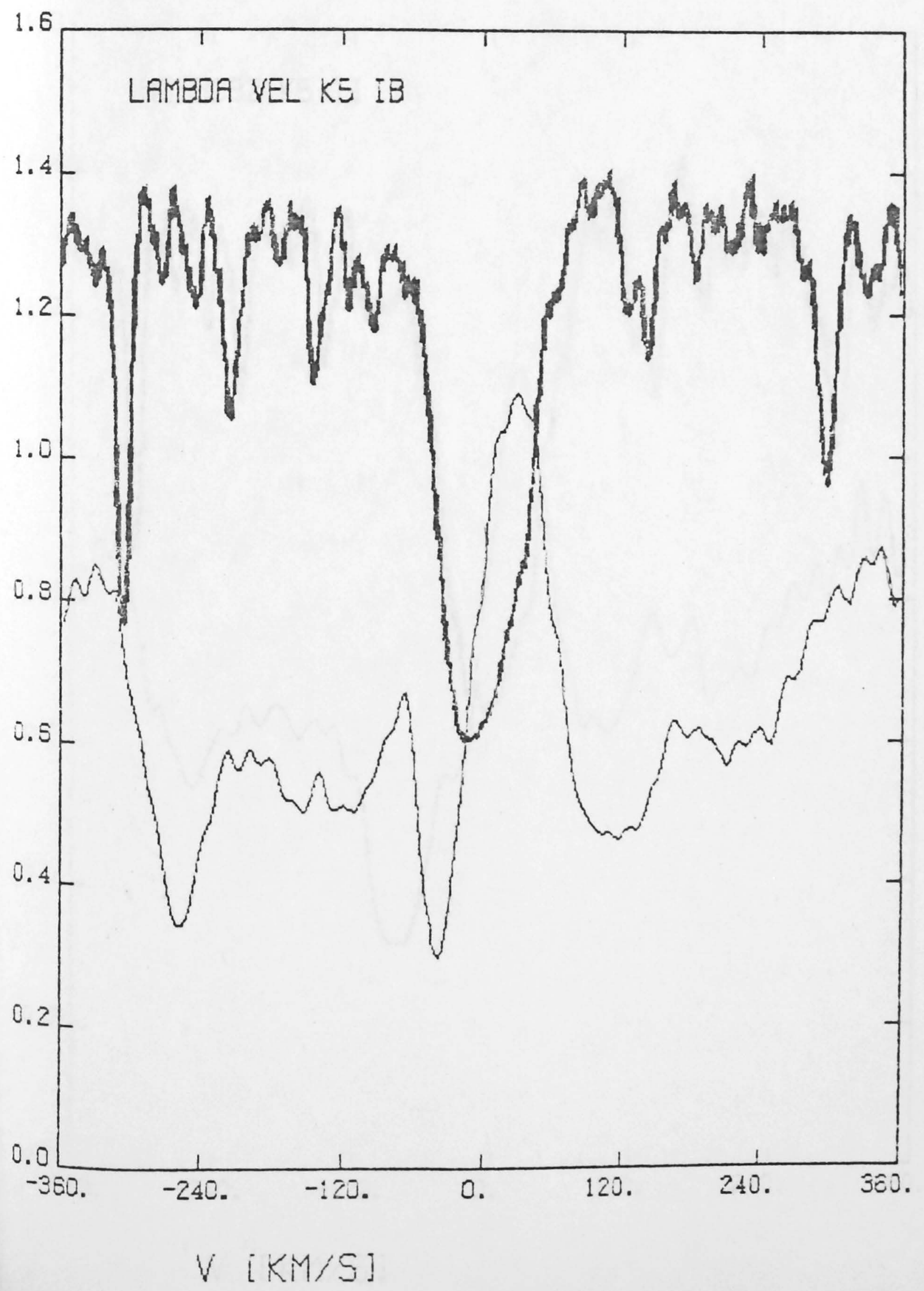




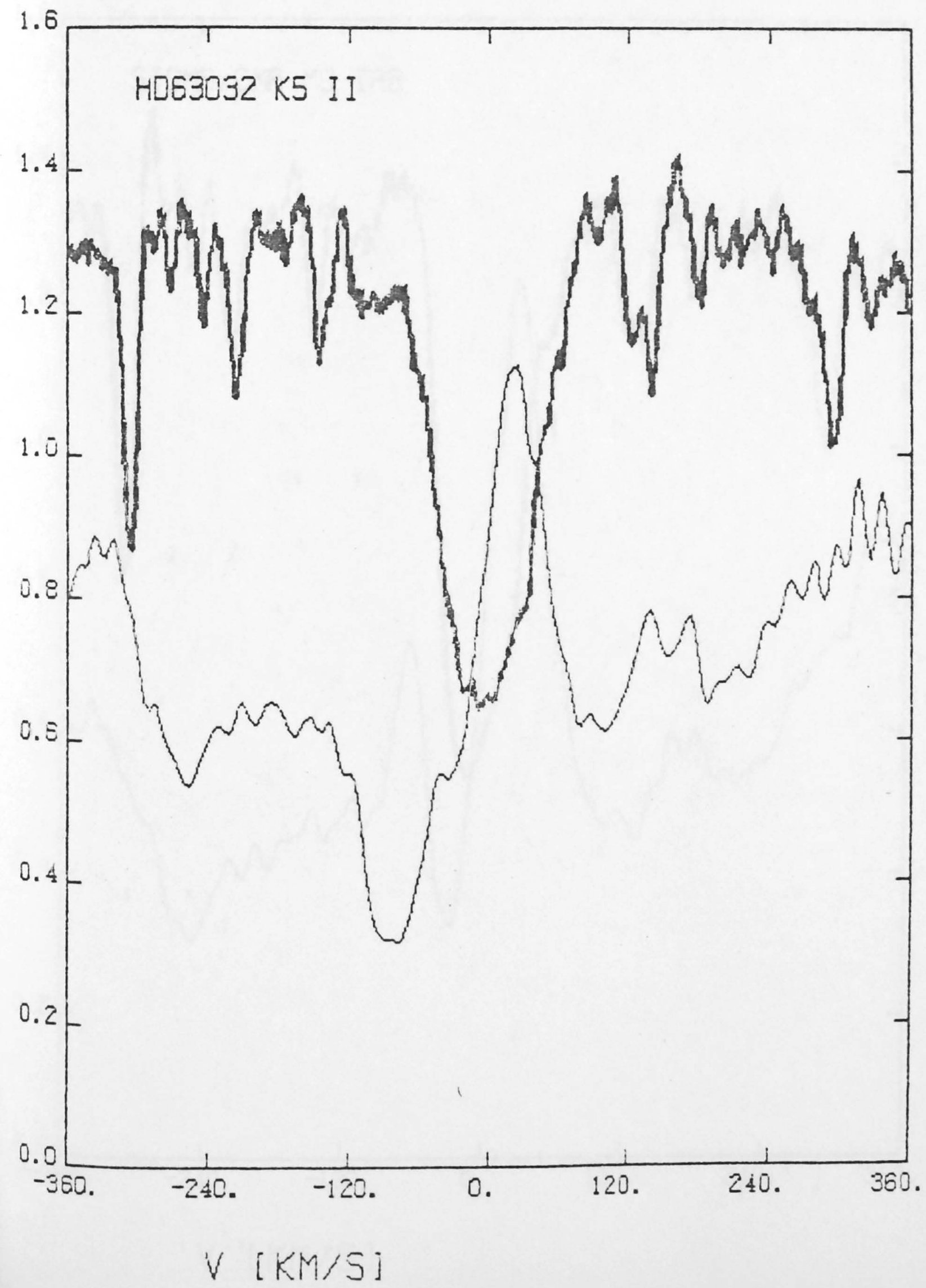




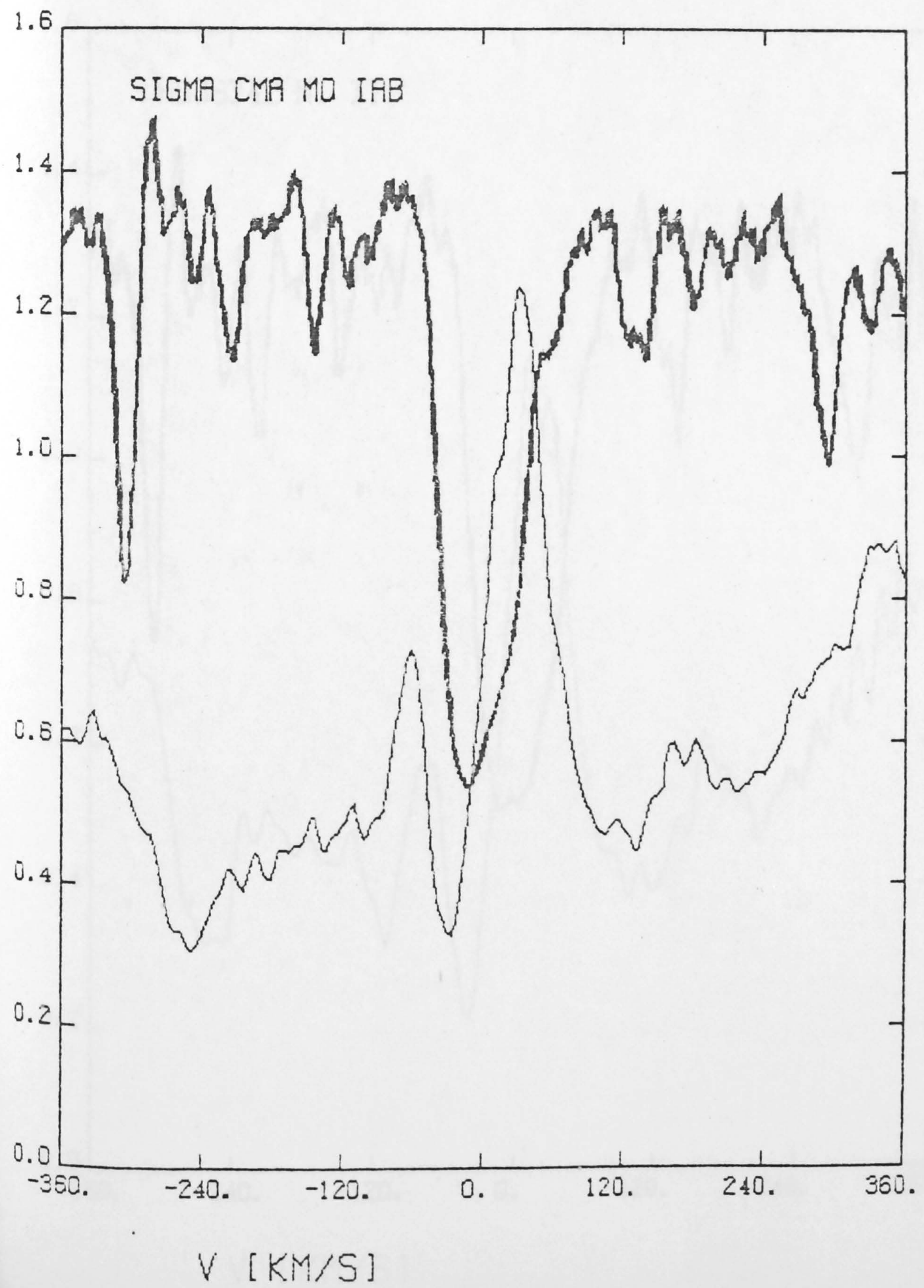




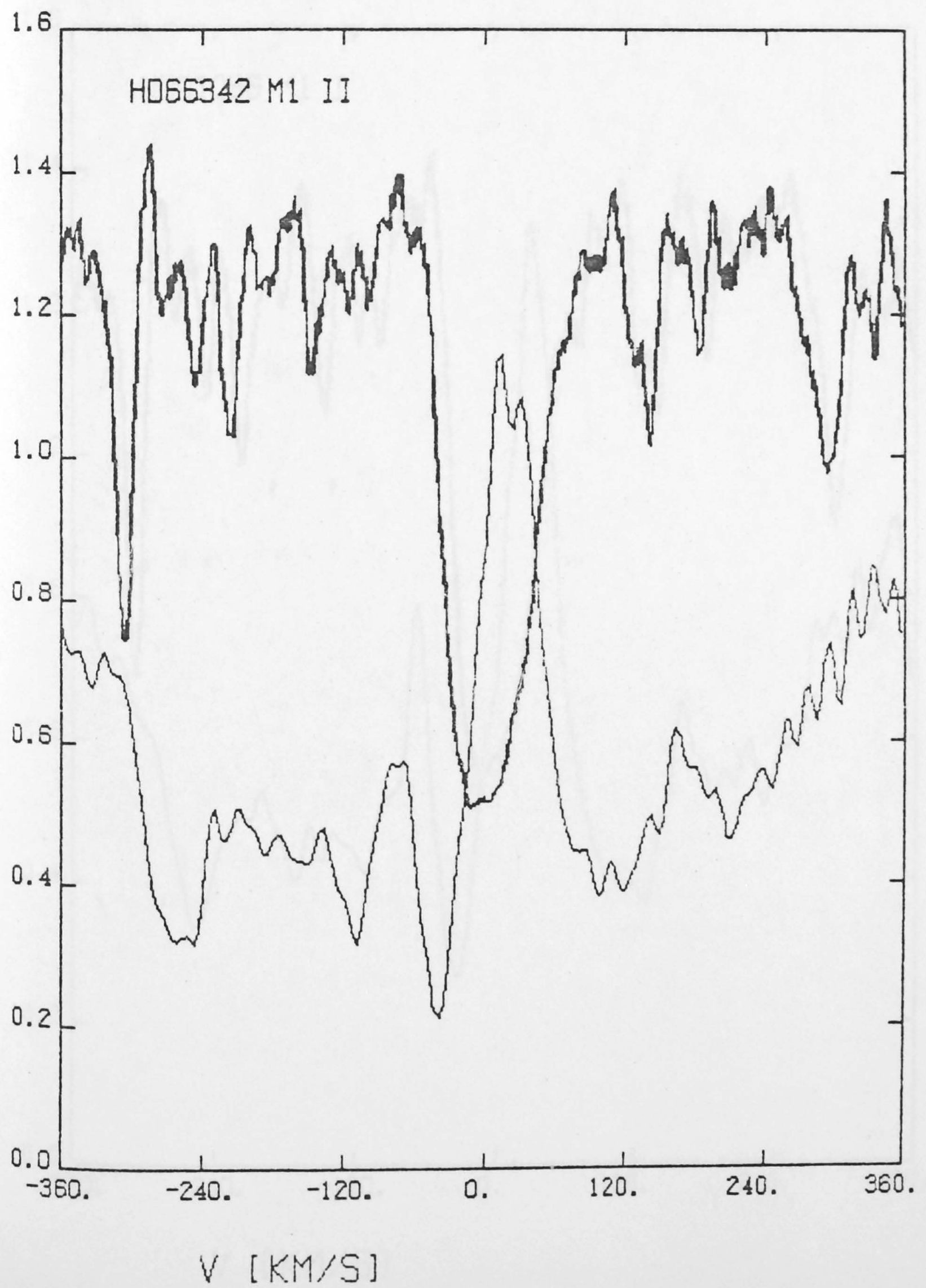




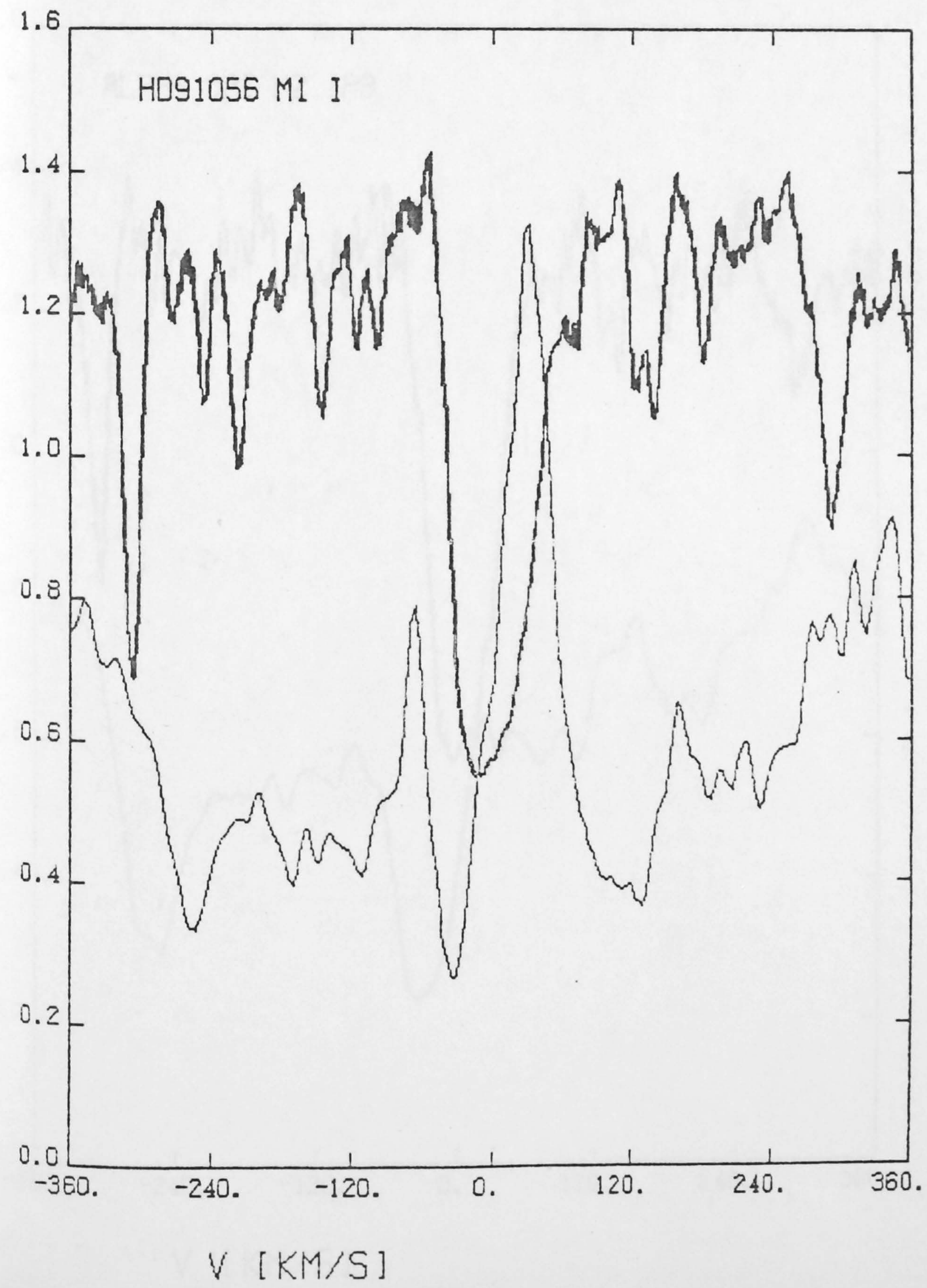




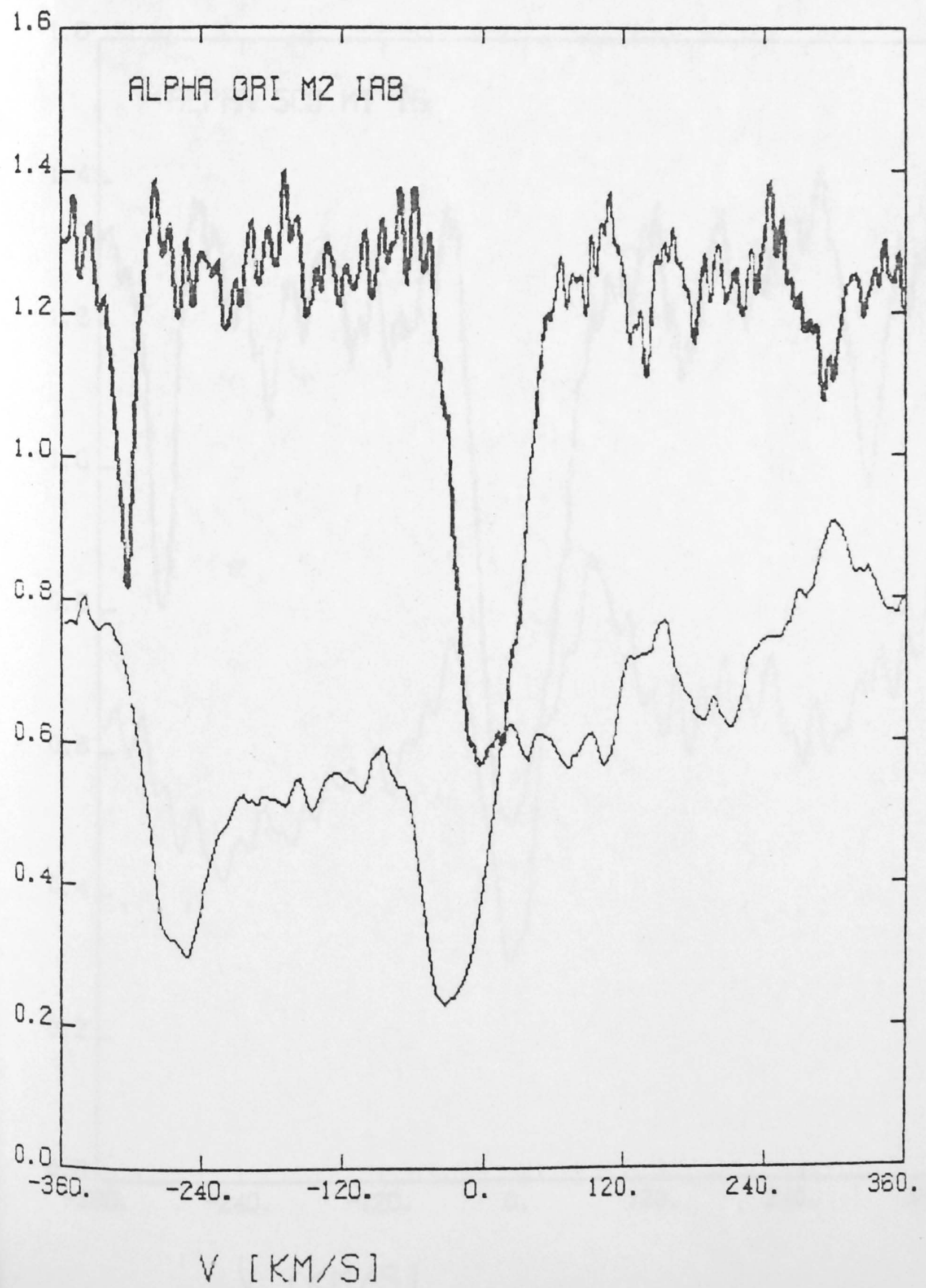




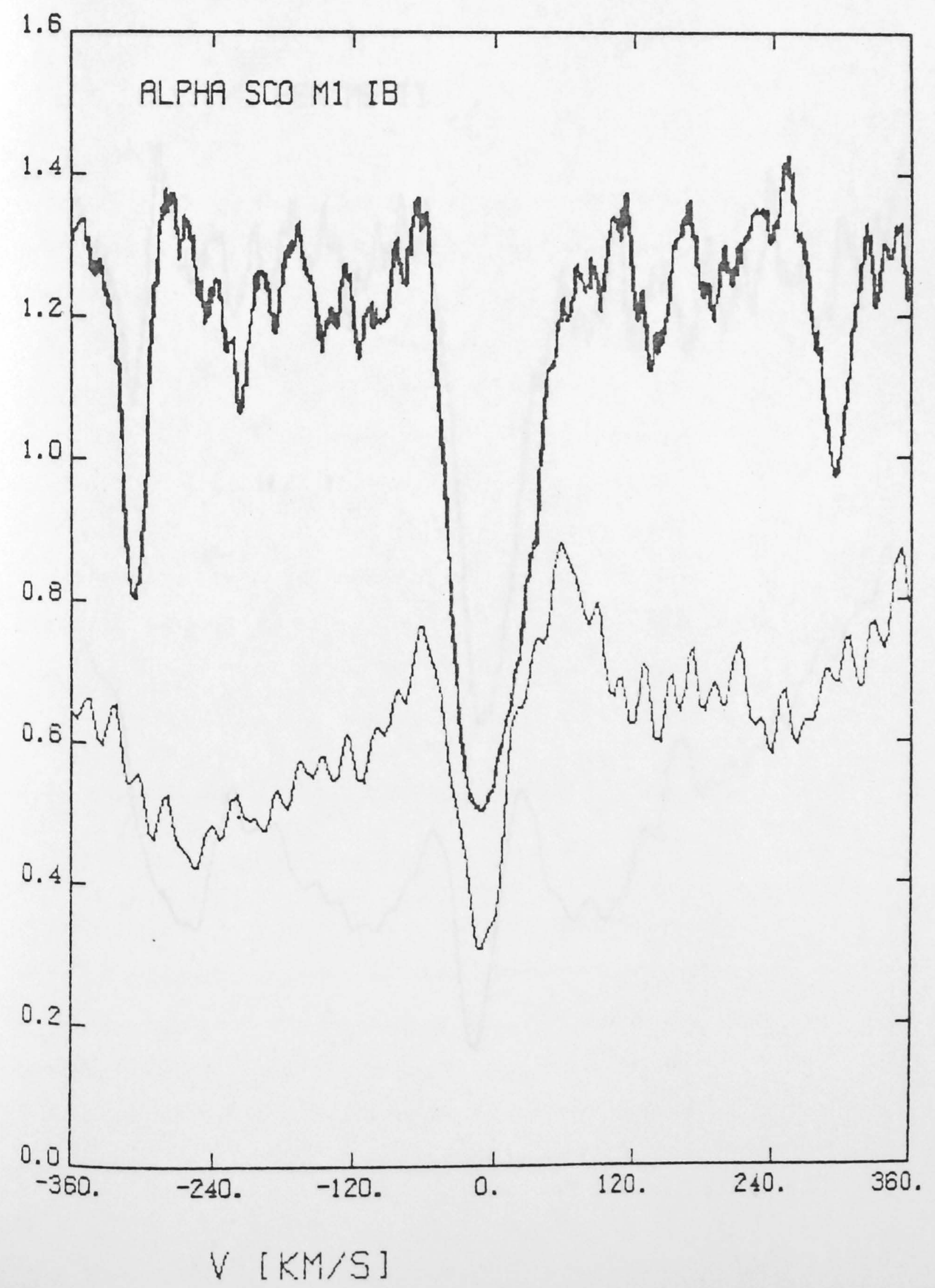




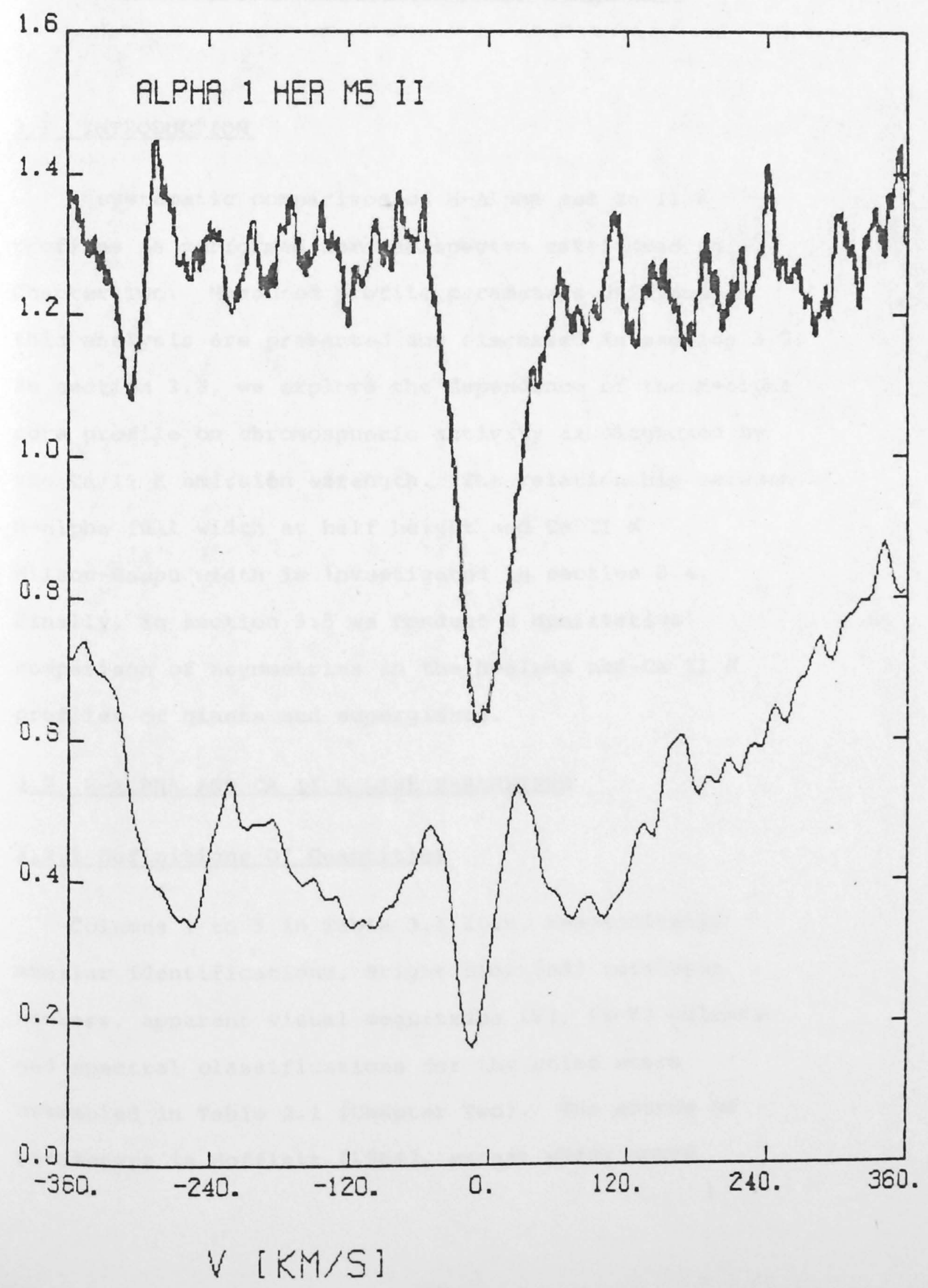




\section{CHAPTER THREE}

\section{ANALYSIS OF H-ALPHA AND CA II K PROFILES}

\subsection{INTRODUCTION}

A systematic comparison of $\mathrm{H}-\mathrm{al}$ pha and $\mathrm{Ca}$ II $\mathrm{K}$ profiles is performed for the spectra catalogued in Chapter Two. Measured profile parameters utilised in this analysis are presented and discussed in section 3.2. In section 3.3 , we explore the dependence of the H-alpha core profile on chromospheric activity as diagnosed by the Ca II $\mathrm{K}$ emission strength. The relationship between H-alpha full width at half height and Ca II K Wilson-Bappu width is investigated in section 3.4 . Finally, in section 3.5 we conduct a qualitative comparison of asymmetries in the $\mathrm{H}$-alpha and $\mathrm{Ca}$ II $\mathrm{K}$ profiles of giants and supergiants.

\subsection{H-ALPHA AND CA II K LINE PARAMETERS}

\subsubsection{Definitions of Quantities}

Columns 1 to 5 in Table 3.1 list, respectively, stellar identifications, Bright Star (BS) catalogue numbers, apparent visual magnitudes $(V),(B-V)$ colours and spectral classifications for the atlas stars assembled in Table 2.1 (Chapter Two). The source of parameters is Hoffleit (1964), except where noted 
TABLE 3.1

MEASURED $\mathrm{H} \propto$ AND $\mathrm{Ca}$ II $\mathrm{K}$ PARAMETERS

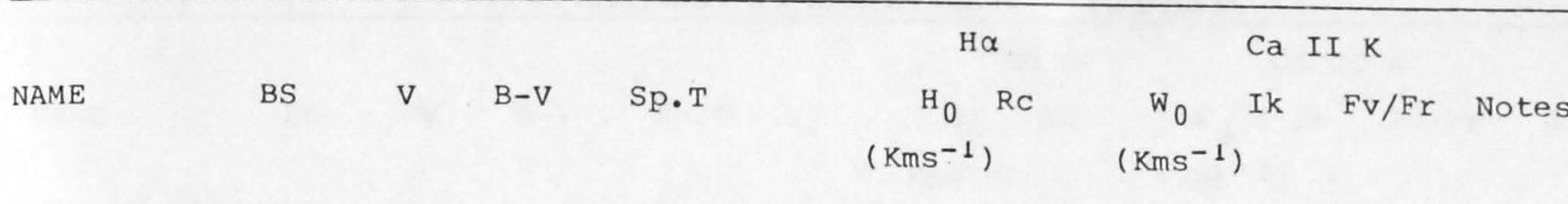

DWARFS

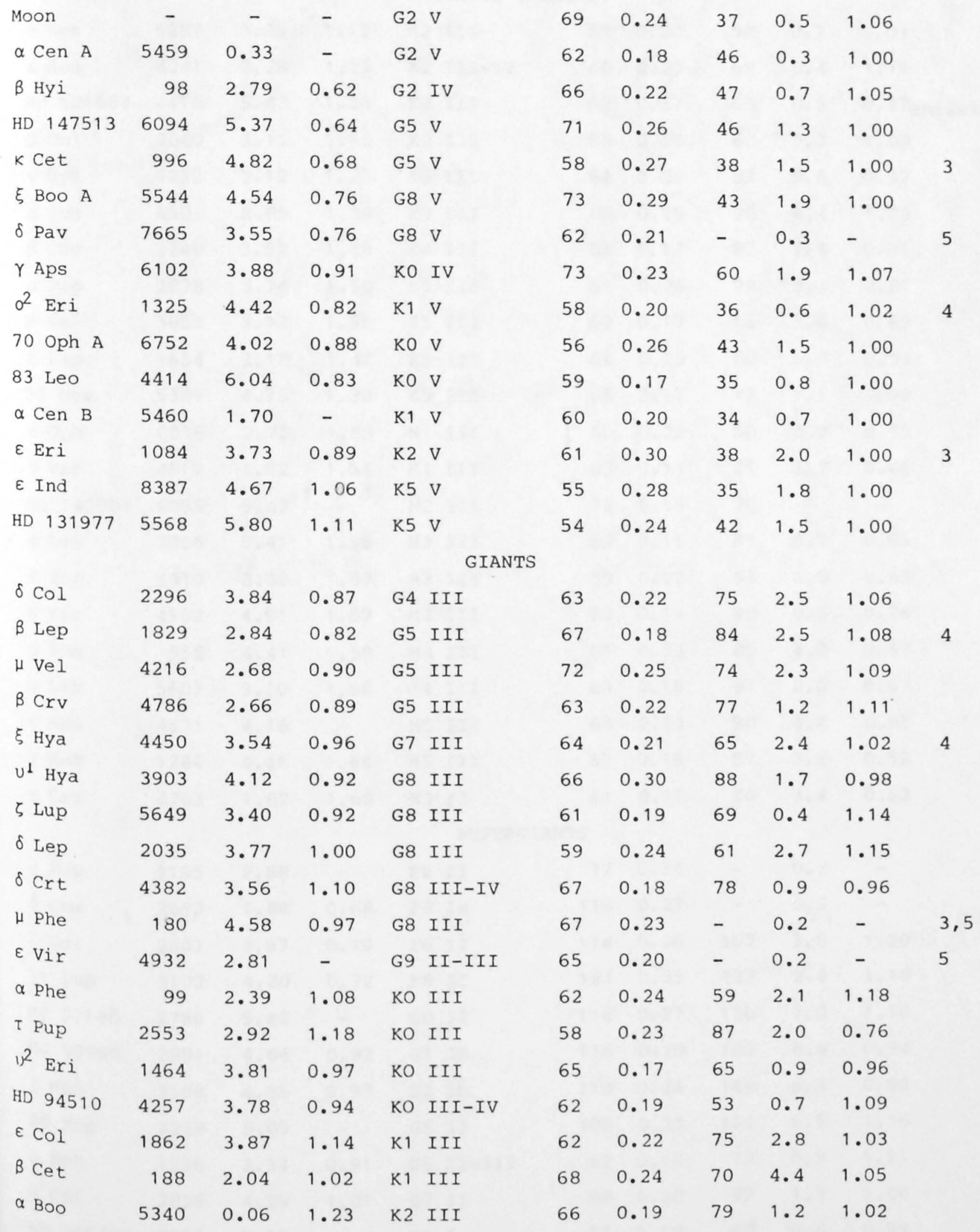


TABLE 3.1

\begin{tabular}{|c|c|c|c|c|c|c|c|c|c|}
\hline \multirow[b]{2}{*}{ NAME } & \multirow[b]{2}{*}{ BS } & \multirow[b]{2}{*}{ V } & \multirow[b]{2}{*}{$B-V$} & \multirow[b]{2}{*}{$\mathrm{Sp} \cdot \mathrm{T}$} & $\mathrm{H} \alpha$ & \multicolumn{4}{|c|}{$\mathrm{Ca}$ II $\mathrm{K}$} \\
\hline & & & & & $\begin{array}{c}\mathrm{H}_{0} \quad \mathrm{RC} \\
\left(\mathrm{Kms}^{-1}\right)\end{array}$ & $\begin{array}{r}\mathrm{W}_{0} \\
(\mathrm{Kms}\end{array}$ & Ik & $\mathrm{Fv} / \mathrm{Fr}$ & Notes \\
\hline
\end{tabular}

\begin{tabular}{|c|c|c|c|c|c|c|c|c|c|c|c|}
\hline \multicolumn{12}{|c|}{ DWARFS } \\
\hline Moon & - & - & - & G2 & V & 69 & 0.24 & 37 & 0.5 & 1.06 & \\
\hline$\alpha \operatorname{Cen} A$ & 5459 & 0.33 & - & G2 & V & 62 & 0.18 & 46 & 0.3 & 1.00 & \\
\hline B Hyi & 98 & 2.79 & 0.62 & G2 & IV & 66 & 0.22 & 47 & 0.7 & 1.05 & \\
\hline HD 147513 & 6094 & 5.37 & 0.64 & G5 & V & 71 & 0.26 & 46 & 1.3 & 1.00 & \\
\hline k Cet & 996 & 4.82 & 0.68 & G5 & $\mathrm{V}$ & 58 & 0.27 & 38 & $1 \cdot 5$ & 1.00 & 3 \\
\hline$\xi$ Boo A & 5544 & 4.54 & 0.76 & G8 & $\mathrm{V}$ & 73 & 0.29 & 43 & 1.9 & 1.00 & \\
\hline$\delta \mathrm{Pav}$ & 7665 & 3.55 & 0.76 & G8 & V & 62 & 0.21 & - & 0.3 & - & 5 \\
\hline$\gamma$ Aps & 6102 & 3.88 & 0.91 & KO & IV & 73 & 0.23 & 60 & 1.9 & 1.07 & \\
\hline $0^{2} \operatorname{Eri}$ & 1325 & 4.42 & 0.82 & $\mathrm{~K} 1$ & $\mathrm{~V}$ & 58 & 0.20 & 36 & 0.6 & 1.02 & 4 \\
\hline 70 Oph A & 6752 & 4.02 & 0.88 & KO & $\mathrm{V}$ & 56 & 0.26 & 43 & 1.5 & 1.00 & \\
\hline 83 Leo & 4414 & 6.04 & 0.83 & KO & V & 59 & 0.17 & 35 & 0.8 & 1.00 & \\
\hline$\alpha$ Cen B & 5460 & 1.70 & - & $\mathrm{K} 1$ & $\mathrm{~V}$ & 60 & 0.20 & 34 & 0.7 & 1.00 & \\
\hline$\varepsilon \operatorname{Eri}$ & 1084 & 3.73 & 0.89 & $\mathrm{~K} 2$ & $\mathrm{~V}$ & 61 & 0.30 & 38 & 2.0 & 1.00 & 3 \\
\hline$\varepsilon$ Ind & 8387 & 4.67 & 1.06 & $\mathrm{~K} 5$ & V & 55 & 0.28 & 35 & 1.8 & 1.00 & \\
\hline HD 131977 & 5568 & 5.80 & 1.11 & K5 & V & 54 & 0.24 & 42 & 1.5 & 1.00 & \\
\hline & & & & & GIAN & & & & & & \\
\hline$\delta \mathrm{Col}$ & 2296 & 3.84 & 0.87 & G4 & III & 63 & 0.22 & 75 & 2.5 & 1.06 & \\
\hline$B$ Lep & 1829 & 2.84 & 0.82 & G5 & III & 67 & 0.18 & 84 & 2.5 & 1.08 & 4 \\
\hline$\mu \mathrm{Vel}$ & 4216 & 2.68 & 0.90 & G5 & III & 72 & 0.25 & 74 & 2.3 & 1.09 & \\
\hline$\beta \mathrm{Crv}$ & 4786 & 2.66 & 0.89 & G5 & III & 63 & 0.22 & 77 & 1.2 & 1.11 & \\
\hline$\xi$ нуа & 4450 & 3.54 & 0.96 & G7 & III & 64 & 0.21 & 65 & $2 \cdot 4$ & 1.02 & 4 \\
\hline$u^{\prime}$ нуа & 3903 & 4.12 & 0.92 & G8 & III & 66 & 0.30 & 88 & 1.7 & 0.98 & \\
\hline$\zeta$ Lup & 5649 & 3.40 & 0.92 & G8 & III & 61 & 0.19 & 69 & 0.4 & 1.14 & \\
\hline$\delta$ Lep & 2035 & 3.77 & 1.00 & G8 & III & 59 & 0.24 & 61 & 2.7 & $1 \cdot 15$ & \\
\hline$\delta \mathrm{Crt}$ & 4382 & 3.56 & 1.10 & G8 & III-IV & 67 & 0.18 & 78 & 0.9 & 0.96 & \\
\hline$\mu$ Phe & 180 & 4.58 & 0.97 & G8 & I I I & 67 & 0.23 & - & 0.2 & - & 3,5 \\
\hline$\varepsilon \operatorname{Vir}$ & 4932 & 2.81 & - & G9 & II-III & 65 & 0.20 & - & 0.2 & - & 5 \\
\hline$\alpha$ Phe & 99 & 2.39 & 1.08 & $\mathrm{KO}$ & III & 62 & 0.24 & 59 & 2.1 & 1.18 & \\
\hline$\tau$ Pup & 2553 & 2.92 & 1.18 & KO & III & 58 & 0.23 & 87 & 2.0 & 0.76 & \\
\hline$u^{2}$ Eri & 1464 & 3.81 & 0.97 & KO & III & 65 & 0.17 & 65 & 0.9 & 0.96 & \\
\hline HD 94510 & 4257 & 3.78 & 0.94 & $\mathrm{KO}$ & III-IV & 62 & 0.19 & 53 & 0.7 & 1.09 & \\
\hline$\varepsilon \mathrm{Col}$ & 1862 & 3.87 & 1.14 & K1 & III & 62 & 0.22 & 75 & 2.8 & 1.03 & \\
\hline B Cet & 188 & 2.04 & 1.02 & K1 & I I I & 68 & 0.24 & 70 & 4.4 & 1.05 & \\
\hline$\alpha$ Boo & 5340 & 0.06 & 1.23 & $\mathrm{~K} 2$ & III & 66 & 0.19 & 79 & 1.2 & 1.02 & \\
\hline
\end{tabular}




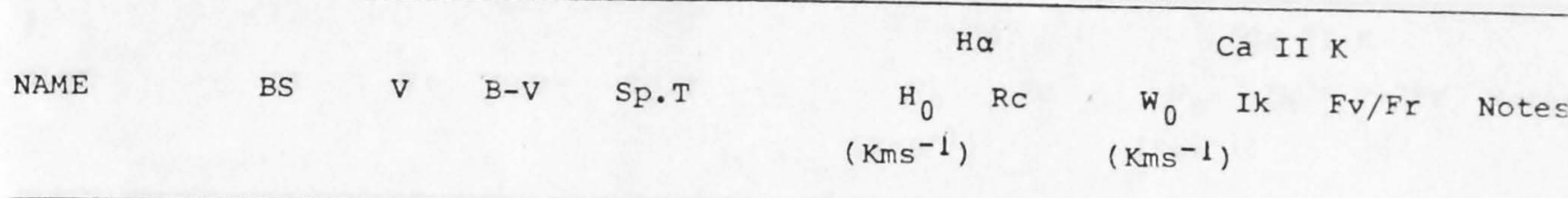

\begin{tabular}{|c|c|c|c|c|c|c|c|c|c|c|c|}
\hline \multicolumn{12}{|c|}{ GIANTS (cont'd) } \\
\hline$\pi$ Hyа & 5287 & 3.25 & 1.12 & $\mathrm{~K} 2$ & III & 59 & 0.20 & 58 & 0.7 & \multicolumn{2}{|l|}{1.01} \\
\hline$\varepsilon \mathrm{Sco}$ & 6241 & 2.28 & 1.15 & $\mathrm{~K} 2$ & III-IV & 60 & 0.22 & 69 & 0.4 & \multicolumn{2}{|l|}{1.11} \\
\hline HD 101067 & 4476 & 5.43 & 1.24 & $\mathrm{~K} 2$ & III & 62 & 0.37 & 60 & 0.5 & \multicolumn{2}{|c|}{0.97 enz ${ }^{\mathrm{H} \alpha} \alpha$ ion } \\
\hline B Col & 2040 & 3.11 & 1.16 & $\mathrm{~K} 2$ & III & 58 & 0.26 & 68 & 0.7 & \multicolumn{2}{|l|}{1.09} \\
\hline$\checkmark$ Hya & 4232 & 3.12 & 1.25 & $\mathrm{~K} 3$ & III & 64 & 0.20 & 83 & 0.8 & \multicolumn{2}{|l|}{0.92} \\
\hline a Tuc & 8502 & 2.85 & 1.39 & $\mathrm{~K} 3$ & III & 62 & 0.19 & 86 & 4.4 & \multicolumn{2}{|l|}{1.09} \\
\hline B Cnc & 3249 & 3.52 & 1.48 & K4 & III & 68 & 0.17 & 87 & 2.8 & \multicolumn{2}{|l|}{0.91} \\
\hline$\sigma$ Pup & 2878 & 3.24 & 1.50 & $\mathrm{~K} 5$ & I II & 61 & 0.26 & 92 & 3.4 & \multicolumn{2}{|l|}{0.97} \\
\hline $\mathrm{N}$ Vel & 3803 & 3.12 & 1.56 & $\mathrm{~K} 5$ & III & 63 & 0.17 & 84 & 3.6 & \multicolumn{2}{|l|}{0.69} \\
\hline$\varepsilon$ Lep & 1654 & 3.18 & 1.47 & K5 & III & 64 & 0.23 & 80 & 6.7 & \multicolumn{2}{|l|}{0.93} \\
\hline 51 Hуа & 5381 & 4.78 & 1.32 & $\mathrm{~K} 5$ & III & 58 & 0.17 & 72 & 1.1 & \multicolumn{2}{|l|}{1.02} \\
\hline$\delta$ Oph & 6056 & 2.72 & 1.58 & M1 & III & 60 & 0.22 & 88 & 4.0 & \multicolumn{2}{|l|}{0.70} \\
\hline$\nu$ vir & 4517 & 4.02 & 1.54 & M1 & I I I & 63 & 0.14 & 87 & 2.1 & \multicolumn{2}{|l|}{0.46} \\
\hline HD 146003 & 6055 & 5.43 & - & M2 & III & 72 & 0.16 & 76 & - & - & 2,6 \\
\hline$\psi$ Leo & 3866 & 5.41 & 1.56 & M2 & III & 59 & 0.15 & 83 & 3.7 & \multicolumn{2}{|l|}{0.65} \\
\hline$\delta$ vir & 4910 & 3.38 & 1.57 & M3 & I I I & 59 & 0.22 & 93 & 4.0 & \multicolumn{2}{|l|}{0.65} \\
\hline$\psi$ vir & 4902 & 4.91 & 1.57 & M3 & III & 63 & 0.14 & 90 & 4.3 & \multicolumn{2}{|l|}{0.78} \\
\hline$\psi$ Phe & 555 & 4.41 & 1.59 & M4 & III & 61 & 0.23 & 85 & 4.7 & 0.93 & 3 \\
\hline$\sigma$ Lib & 5603 & 3.30 & 1.66 & M4 & III & 60 & 0.10 & 87 & 2.6 & \multicolumn{2}{|l|}{0.67} \\
\hline$\varepsilon$ Mus & 4671 & 4.16 & - & M5 & I I I & 64 & 0.14 & 90 & 4.4 & \multicolumn{2}{|l|}{0.95} \\
\hline$\gamma \operatorname{Ret}$ & 1264 & 4.46 & 1.66 & M5 & III & 55 & 0.19 & 87 & 3.2 & \multicolumn{2}{|l|}{0.52} \\
\hline$\gamma \mathrm{Cru}$ & 4763 & 1.62 & 1.60 & M3 & II & 61 & 0.17 & 90 & 3.4 & 0.62 & 4 \\
\hline \multicolumn{12}{|c|}{ SUPERGIANTS } \\
\hline P Pup & 3185 & 2.88 & - & F6 & II & 77 & 0.34 & - & 0.3 & - & 5 \\
\hline$\delta \mathrm{Cma}$ & 2693 & 1.84 & 0.68 & F8 & Ia & 116 & 0.27 & - & 0.3 & - & 5 \\
\hline$\delta$ Vol & 2803 & 3.97 & 0.79 & F6 & II & 114 & 0.26 & 107 & 3.6 & 1.10 & 1,4 \\
\hline 11 Pup & 3102 & 4.20 & 0.72 & F8 & II & 124 & 0.35 & 137 & 2.4 & 1.18 & 4 \\
\hline HD 57146 & 2786 & 5.40 & - & GO & II & 116 & 0.27 & 130 & 7.0 & 1.10 & 4 \\
\hline HD 59890 & 2881 & 4.64 & 0.92 & G1 & Ib & 116 & 0.29 & 163 & 6.8 & 0.94 & \\
\hline$\zeta$ Mon & 3188 & 4.35 & 0.97 & $\mathrm{G} 2$ & Ib & 110 & 0.24 & 149 & 6.3 & 0.90 & 3,4 \\
\hline 20 Pup & 3229 & 5.05 & - & G5 & II & 105 & 0.23 & 124 & 6.5 & 1.16 & 3 \\
\hline$\alpha \operatorname{Ret}$ & 1336 & 3.34 & 0.91 & G8 & II-III & 62 & 0.18 & 79 & 0.9 & 1.21 & 1 \\
\hline$\varepsilon \mathrm{Cmi}$ & 2828 & 4.99 & 1.01 & G7 & II & 88 & 0.20 & 92 & 1.3 & 1.06 & 1 \\
\hline HD 165438 & 6756 & 5.90 & - & K1 & I & 59 & 0.18 & 42 & 0.4 & 0.98 & 6 \\
\hline HD 93070 & 4200 & 4.56 & 1.72 & $\mathrm{~K} 3$ & Ib & 89 & 0.20 & 87 & - & - & 1,2 \\
\hline
\end{tabular}




\begin{tabular}{|c|c|c|c|c|c|c|c|c|c|c|}
\hline \multirow[b]{2}{*}{ NAME } & \multirow[b]{2}{*}{ BS } & \multirow[b]{2}{*}{ V } & \multirow[b]{2}{*}{$B-V$} & \multirow[b]{2}{*}{$\mathrm{Sp} . \mathrm{T}$} & \multicolumn{2}{|c|}{$\mathrm{H} \alpha$} & \multicolumn{4}{|c|}{$\mathrm{Ca}$ II K } \\
\hline & & & & & $\begin{array}{r}{ }^{\mathrm{H}_{0}} \\
\left(\mathrm{Kms}^{-}\right.\end{array}$ & $\mathrm{Rc}$ & $\begin{array}{r}w_{0} \\
(\mathrm{Kms}\end{array}$ & Ik & $\mathrm{Fv} / \mathrm{Fr}$ & Notes \\
\hline
\end{tabular}

\begin{tabular}{|c|c|c|c|c|c|c|c|c|c|c|c|}
\hline & & & & SUPE & ERGIANTS & ont'd & & & & & \\
\hline HD 65662 & 3120 & 5.73 & 1.55 & K4 & I I & 75 & 0.19 & 97 & 3.1 & 1.05 & 1 \\
\hline HD 152781 & 6284 & 6.49 & - & $\mathrm{K} 2$ & I & 59 & 0.17 & 59 & 0.5 & 1.00 & 6 \\
\hline$\nu^{1} \mathrm{sgr}$ & 7116 & 4.83 & 1.42 & K2 & I & 150 & 0.22 & 120 & - & - & 2,4 \\
\hline HD 68553 & 3225 & 4.44 & 1.63 & K3 & Ib & 102 & 0.24 & 98 & - & - & 1,2 \\
\hline HD 91942 & 4159 & 4.44 & 1.63 & K3 & II I & 99 & 0.16 & 96 & - & - & 2,6 \\
\hline B Ara & 6461 & 2.84 & 1.46 & $\mathrm{~K} 3$ & Ib & 108 & 0.19 & 155 & 7.1 & 0.73 & \\
\hline$\gamma$ Phe & 429 & 3.40 & 1.57 & K5 & II & 78 & 0.20 & 85 & - & - & 2 \\
\hline$\pi$ Pup & 2773 & 2.70 & 1.63 & K5 & I I I & 83 & 0.21 & 154 & 8.2 & 0.72 & 6 \\
\hline$\alpha \operatorname{Tr} A$ & 6217 & 1.91 & 1.44 & $\mathrm{~K} 2$ & II-III & 102 & 0.23 & 121 & 6.1 & 0.99 & 1 \\
\hline HD 89388 & 4050 & 3.44 & 1.54 & $\mathrm{~K} 3$ & II & 105 & 0.20 & 105 & - & - & 1,2 \\
\hline$\lambda$ Vel & 3634 & 2.30 & 1.70 & $\mathrm{~K} 5$ & Ib & 86 & 0.21 & 140 & 9.7 & 0.61 & \\
\hline HD 63032 & 3017 & 3.60 & 1.73 & K5 & I I & 96 & 0.25 & 95 & - & - & 1,2 \\
\hline$\sigma \mathrm{Cma}$ & 2646 & 3.46 & 1.74 & MO & Iab & 78 & 0.14 & 130 & 9.3 & 0.59 & \\
\hline HD 66342 & 3153 & 5.16 & 1.72 & M1 & II & 86 & 0.12 & 139 & 8.3 & 0.50 & 1 \\
\hline HD 91056 & 4120 & 5.26 & 1.86 & M1 & I & 79 & 0.15 & 128 & 10.3 & 0.58 & 6 \\
\hline$\alpha$ ori & 2061 & 0.80 & 1.86 & M2 & I ab & 68 & 0.17 & 156 & 6.4 & 0.95 & 3 \\
\hline$\alpha \operatorname{Sco}$ & 6134 & 1.08 & 1.80 & M1 & Ib & 81 & 0.10 & 163 & 10.9 & 0.86 & \\
\hline$\alpha^{1}$ Her & 6406 & 3.10 & - & M5 & II & 62 & 0.23 & 112 & 4.2 & 0.89 & \\
\hline
\end{tabular}

Notes. 1

Spectral type revised from that given in Bright Star Catalogue (BSC) according to Kennedy (1981).

2 Due to significant absorption at $\mathrm{K}_{1 \mathrm{v}}, \mathrm{w}_{0}$ is derived by doubling distance between midpoint of outer red edge of K2and line centre.

3 Flatfield exposures less than optimum quality for $H \alpha$.

4 Signal to noise ratio for $C a$ II $K$ less than optimum due to poor skies.

5 CaII $K$ emission too weak for accurate width measurement.

6 Probably doubtful luminosity classification from BSC. 
otherwise in Table 3.1 (notes 1,6). Figure 3.1 displays schematic $\mathrm{H}-\mathrm{al}$ pha and $\mathrm{Ca}$ II K spectra, juxtaposed as they are presented in the atlas. On these profiles we

illustrate definitions of various quantities tabulated in the remaining columns of Table 3.1 .

For H-alpha, Rc denotes the minimum residual flux in the line relative to the adjudged continuum level. In the case of asymmetrical H-alpha cores, the wavelength at which Rc is measured is not necessarily at the apparent photospheric rest wavelength of $\mathrm{H}-\mathrm{alpha}$ (adopted as 6562.808; Moore et al., 1966). At a level midway between the estimated continuum and the minimum residual flux in the core, we determine the $\mathrm{H}-\mathrm{al}$ pha full width which is designated Ho $[\mathrm{km} / \mathrm{s}]$.

For the Ca II K emission profile, the FWHM (Wo, $\mathrm{km} / \mathrm{s}$ ) is derived as shown in Figure 3.1. This parameter corresponds closely to the Wilson-Bappu (W-B) width. In the case of asymmetrical lines, wo is measured between the outer midpoints of the violet and red edges of the emission components. These midpoints are defined according to the mean of counts between $\mathrm{Kl}$ and $\mathrm{K} 2$, where $\mathrm{Kl}$ is located at the base of the outer edge of the emission profile, and $\mathrm{K} 2$ at the corresponding maximum in the emission component. When an absorption feature (designated $\mathrm{K} 3$ ) is evident in the $\mathrm{K}$ emission profile, we evaluate an asymmetry index from the ratio of peak fluxes (Fv/Fr) for the violet and red components (stencel, 1978). Additionally, we compute a Ca II K emission strength index ( $I k$ ) by planimetering the emission core 


\section{FIGURE 3.1}

Idealised spectra of $\mathrm{H}-a l p h a$ and $\mathrm{Ca}$ II $\mathrm{K}$ illustrating the definition of 1 ine parameters we have measured. $\mathrm{H}_{0}$ is the full width at half central depth of $\mathrm{H}-\mathrm{alpha}$ and $\mathrm{Rc}$ is the central residual flux measured relative to the continuum. $W_{0}$ is the standard $\mathrm{Ca}$ II $\mathrm{K}$ emission reversal width used in the Wilson-Bappu relation. In the case of asymmetrical profiles, $\mathrm{W}_{0}$ is measured between the outer midpoints of the violet and red edges of the Ca II $\mathrm{K}$ emission components. 


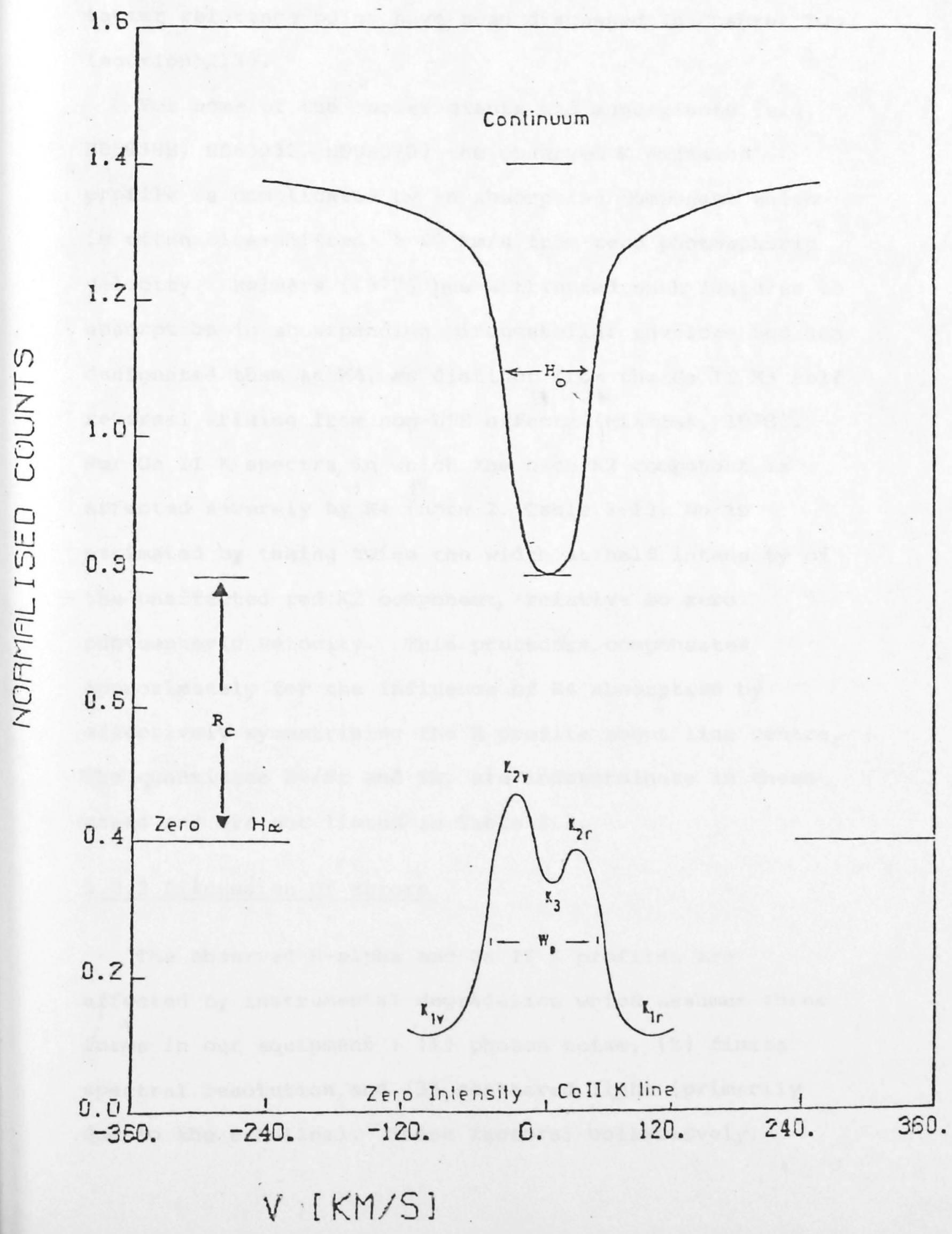


between zero count level and the Kl minima, and normalising with respect to the counts in the adjacent line wing at $3939.682 \AA$. Reasons for the adoption of the latter reference point have been discussed in Chapter Two ( section 2.3).

For some of the cooler giants and supergiants (e.g. HD89388, HD63032, HD93070) the observed K emission profile is complicated by an absorption component which is often blue-shifted > $80 \mathrm{~km} / \mathrm{s}$ from zero photospheric velocity. Reimers (1977) has attributed such features to absorption in an expanding circumstellar envelope and has designated them as $\mathrm{K} 4$, as distinct from the Ca II K3 self reversal arising from non-LTE effects (Mihalas, 1978). For Ca II K spectra, in which the blue K2 component is affected severely by K4 (note 2, Table 3.1), Wo is estimated by taking twice the width at half intensity of the unaffected red $\mathrm{K} 2$ component, relative to zero photospheric velocity. This procedure compensates approximately for the influence of $K 4$ absorption by effectively symmetrising the $\mathrm{K}$ profile about line centre. The quantities Fv/Fr and Ik, are indeterminate in these cases and are not listed in Table 3.1.

\subsubsection{Discussion of Errors}

The observed $\mathrm{H}$-alpha and $\mathrm{Ca}$ II $\mathrm{K}$ profiles are affected by instrumental degradation which assumes three forms in our equipment : (1) photon noise, (2) finite spectral resolution, and (3) scattered light (primarily due to the echelles). These factors, collectively, 
introduce errors into our line parameter measurements. The ratio quantities Rc, Fv/Fr and, in particular, Ik are susceptible to variations in scattered light levels. The errors introduced by such variations are difficult to ascertain.

For $\mathrm{H}-\mathrm{al}$ pha and $\mathrm{Ca}$ II $\mathrm{K}$, mean spectral broadenings of 9 and $16 \mathrm{~km} / \mathrm{s}$, respectively, have been estimated (Chapter Two, section 2.3); effects of scattered light on measured line widths are small in the case of $\mathrm{H}$-alpha, but more pronounced at Ca II K. We use the above values to correct quadratically the raw widths compiled in Table 3.1 according to the formula,

$$
\text { Wo }^{\operatorname{cor} r}=\left(\mathrm{WO}^{2}-\delta^{2}\right) 1 / 2
$$

where $\delta$ is the corresponding profile broadening parameter.

Errors in Ho can arise from uncertainties in the location of the continuum, which becomes increasingly difficult to assign in many of the cool, luminous stars containing numerous photospheric absorption lines (e.g. Gamma Ret [M4 III]). We contend, however, that an uncertainty in the continuum intensity level propagates into only a small error in Ho. For Ca II K, low signal to noise at the edges of the core emission (notes 4,5 in Table 3.1) and the appearance of $\mathrm{K} 4$ features in many of the cooler giants, can lead to considerable inaccuracy in the location of $\mathrm{Kl}$ and, consequently, a large error in Wo.

In order to assess the quality of our width 
determinations, we compare several of our measurements with previously published values. Figure 3.2 a compares instrumentally corrected Ho widths with corresponding measurements of Kraft et al. (1964) for 15 stars overlapping with our sample. A similar comparison is effected in Figure 3.2b, using 57 common wo measurements from Wilson (1976). Many of the corrected wo widths tend to lie above the corresponding values of Wilson (1976). This suggests that the estimated $16 \mathrm{~km} / \mathrm{s}$ correction factor may be too low.

Based on the data points in these diagrams, we compute standard deviations of 6 and $16 \mathrm{~km} / \mathrm{s}$ for to and Wo, respectively. We adopt these values as indications of the errors associated with our width measurements (relative to the previously cited published values). We caution that values of Wo derived by symmetrising the $\mathrm{Ca}$ II $K$ profile probably will have attached errors in excess of $16 \mathrm{~km} / \mathrm{s}$.

\subsection{H-ALPHA CORE INTENSITY AND CHROMOSPHERIC ACTIVITY}

The importance of the Ca II $\mathrm{K}$ emission strength as an indicator of chromospheric activity has been affirmed in studies of solar plages (Shine and Linsky, 1974) and of late type active dwarfs (Kelch et al., 1979). In this section, we exploit this diagnostic capability in an analysis of the H-alpha core residual flux among stars of differing Ca II K brightnesses. Linsky and Ayres (1978) point out that it is not the apparent $\mathrm{Ca}$ II $\mathrm{K}$ intensity which distinguishes stellar chromospheric activity, but 
FIGURE $3.2 \mathrm{a}$

A comparison of corrected Ho for 15 atlas stars (vertical axis) with corresponding measurements of kraft et al. (1964) (horizontal axis). The data are plotted on a LogLog scale. 


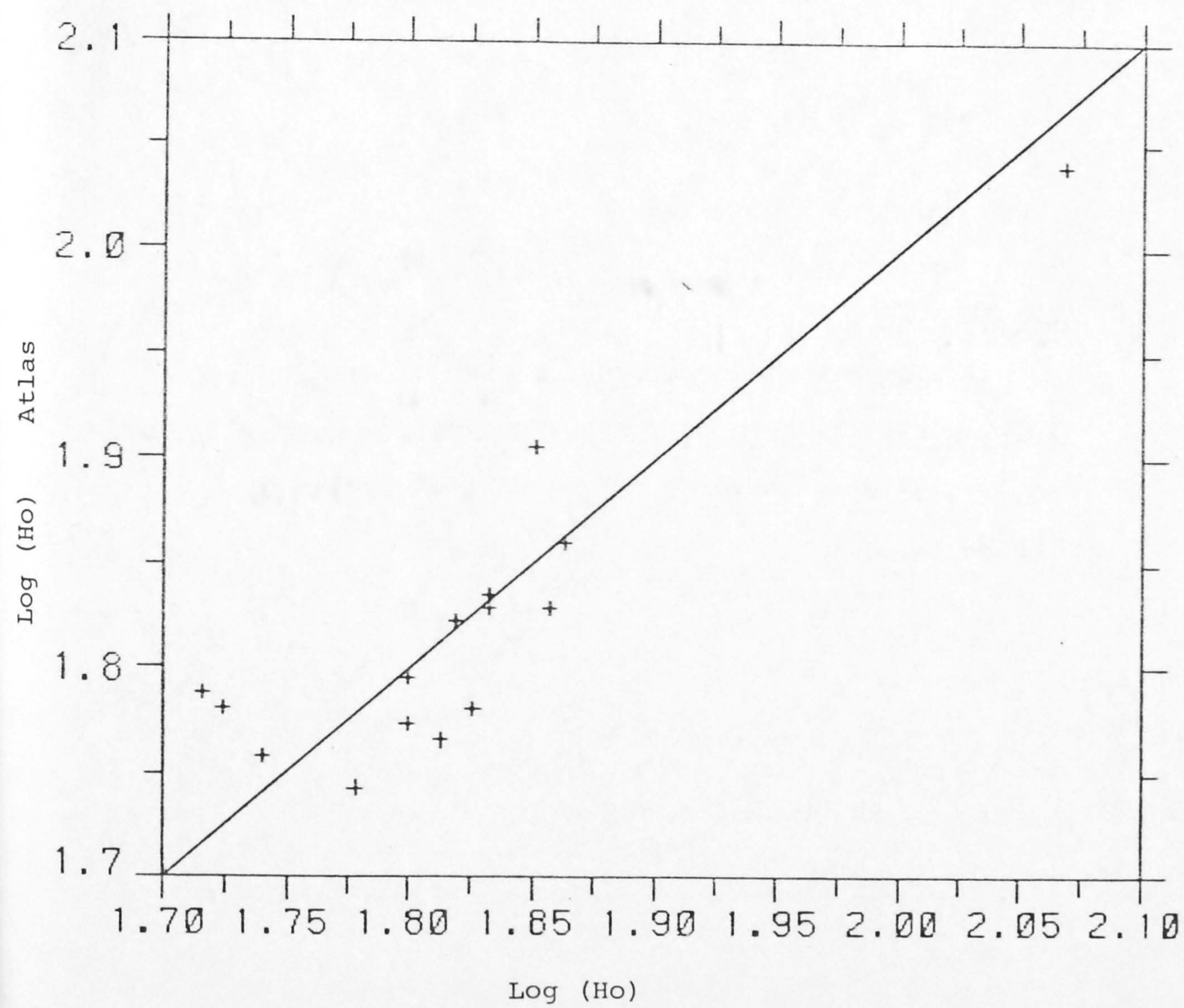

Kraft et al. (1964) 


\section{FIGURE $3.2 \mathrm{~b}$}

A comparison of corrected Wo for 57 atlas stars (vertical axis) with corresponding measurements of Wilson (1976) (horizontal axis). The data are plotted on a Log-Log scale. 


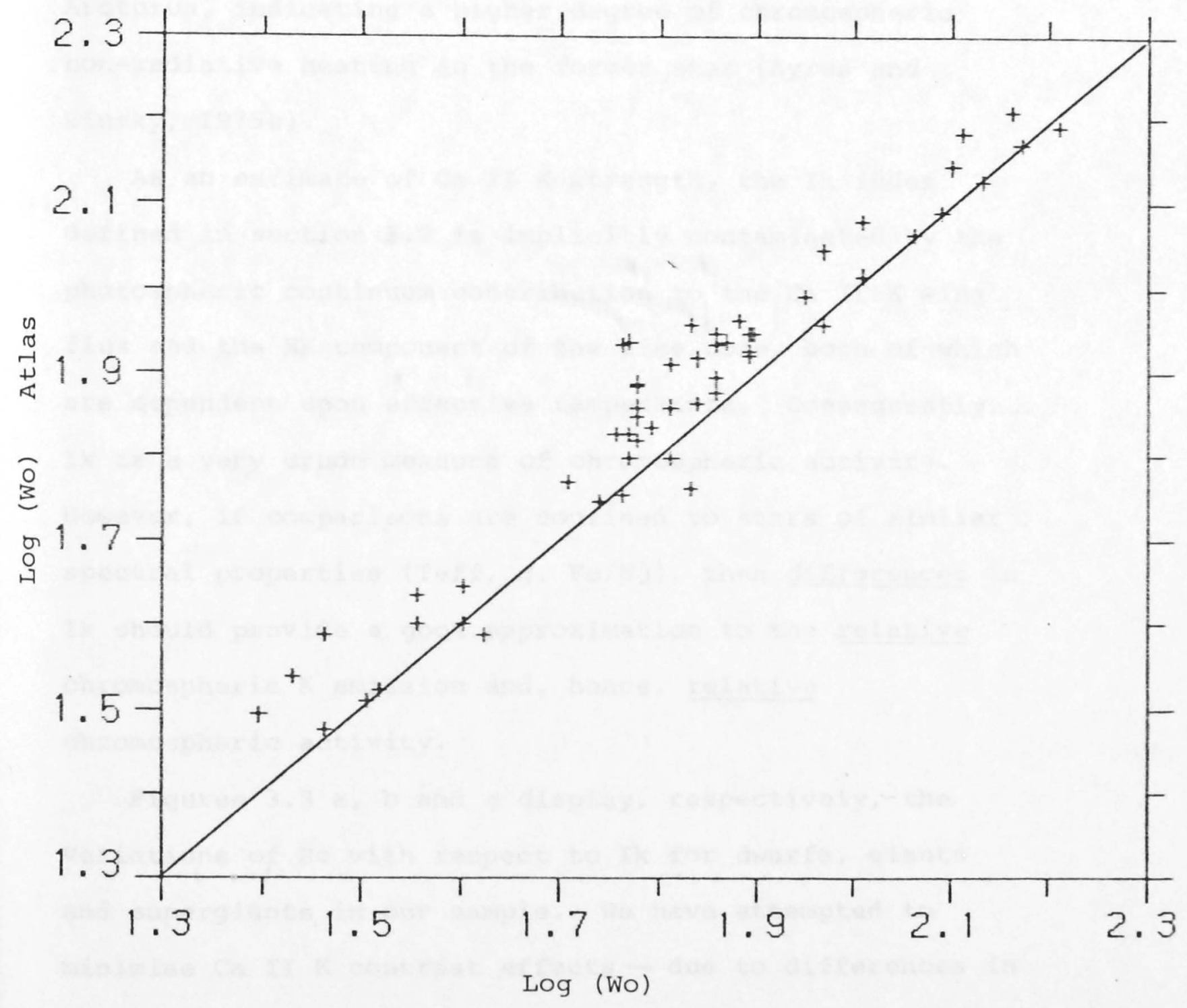

Wilson (1976) 
rather the intrinsic chromospheric radiative energy line

loss. They cite the contrasting examples of the

integrated Sun and Arcturus, the former displaying

apparently weaker $\mathrm{K}$ emission than the latter. After

correction for the photospheric radiative equilibrium

(RE) component of the $\mathrm{K}$ line core, the chromospheric $\mathrm{Ca}$

II $K$ loss in the sun is found to be much larger than for

Arcturus, indicating a higher degree of chromospheric

non-radiative heating in the former star (Ayres and

Linsky, 1975b).

As an estimate of Ca II $K$ strength, the IK index

defined in section 3.2 is implicitly contaminated by the photospheric continuum contribution to the Ca II $K$ wing flux and the RE component of the line core, both of which are dependent upon effective temperature. Consequently, Ik is a very crude measure of chromospheric activity. However, if comparisons are confined to stars of similar spectral properties (Teff, $g, F e / H])$, then differences in Ik should provide a good approximation to the relative chromospheric $\mathrm{K}$ emission and, hence, relative chromospheric activity.

Figures $3.3 \mathrm{a}, \mathrm{b}$ and $\mathrm{c}$ display, respectively, the variations of Rc with respect to Ik for dwarfs, giants and supergiants in our sample. We have attempted to minimise $\mathrm{Ca}$ II $\mathrm{K}$ contrast effects - due to differences in effective temperature - by binning the stars according to G, $K$ and $M$ spectral types. The solid lines drawn are meant solely to indicate rough trends in the data. 
FIGURE $3.3 \mathrm{a}$

Rc as a function of Ik for dwarfs. Stars are binned according to $\mathrm{G}(+), \mathrm{K}$ ( ) spectral types. Note the enhancement in Rc with increasing Ik among the $\mathrm{G}$ and $\mathrm{K}$ dwarfs. 


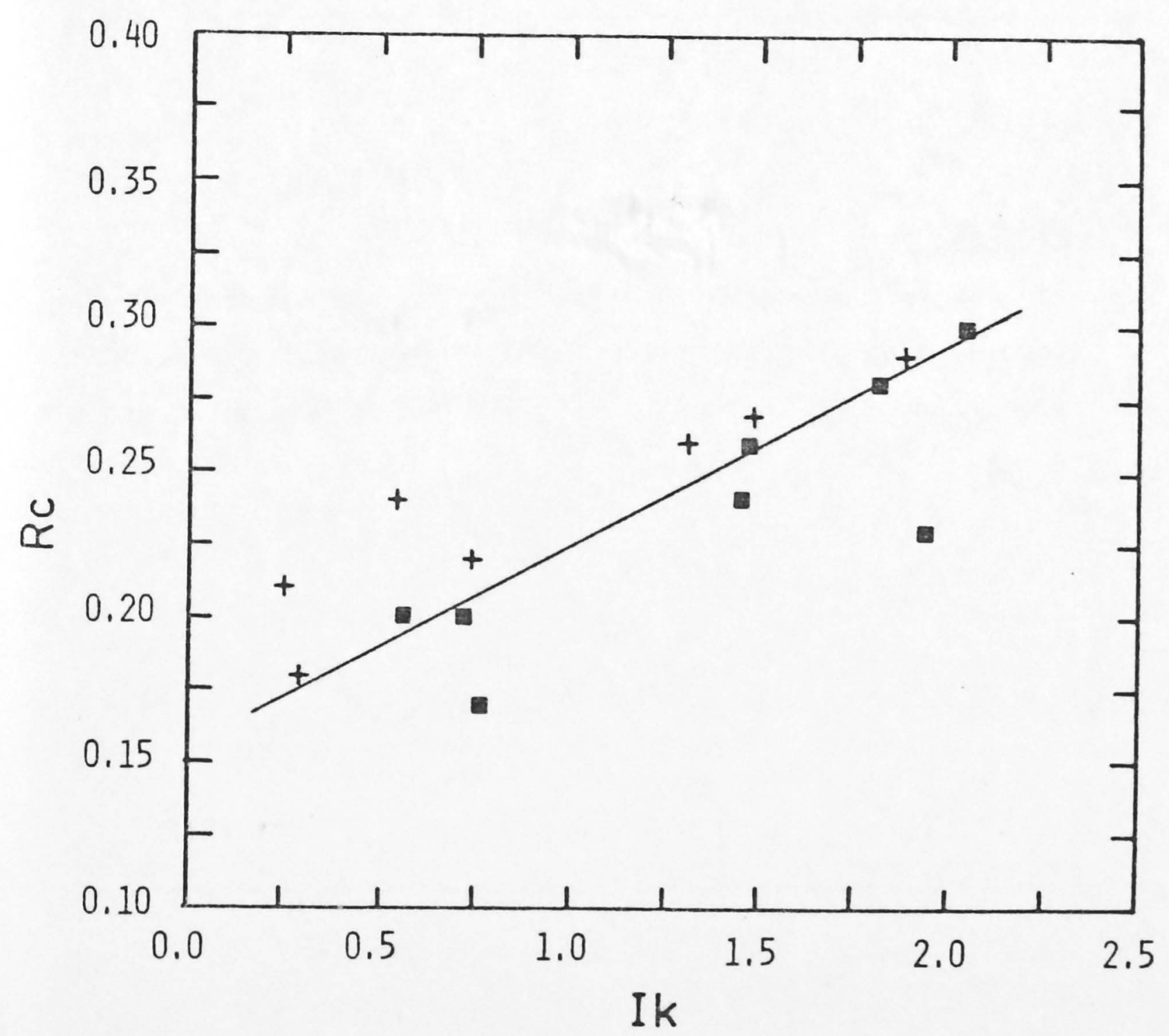




\section{FIGURE $3.3 \mathrm{~b}$}

RC as a function of Ik for giants. Stars are binned according to $G(+), K\left({ }^{*}\right)$ and $M\left({ }^{*}\right)$ spectral types. 


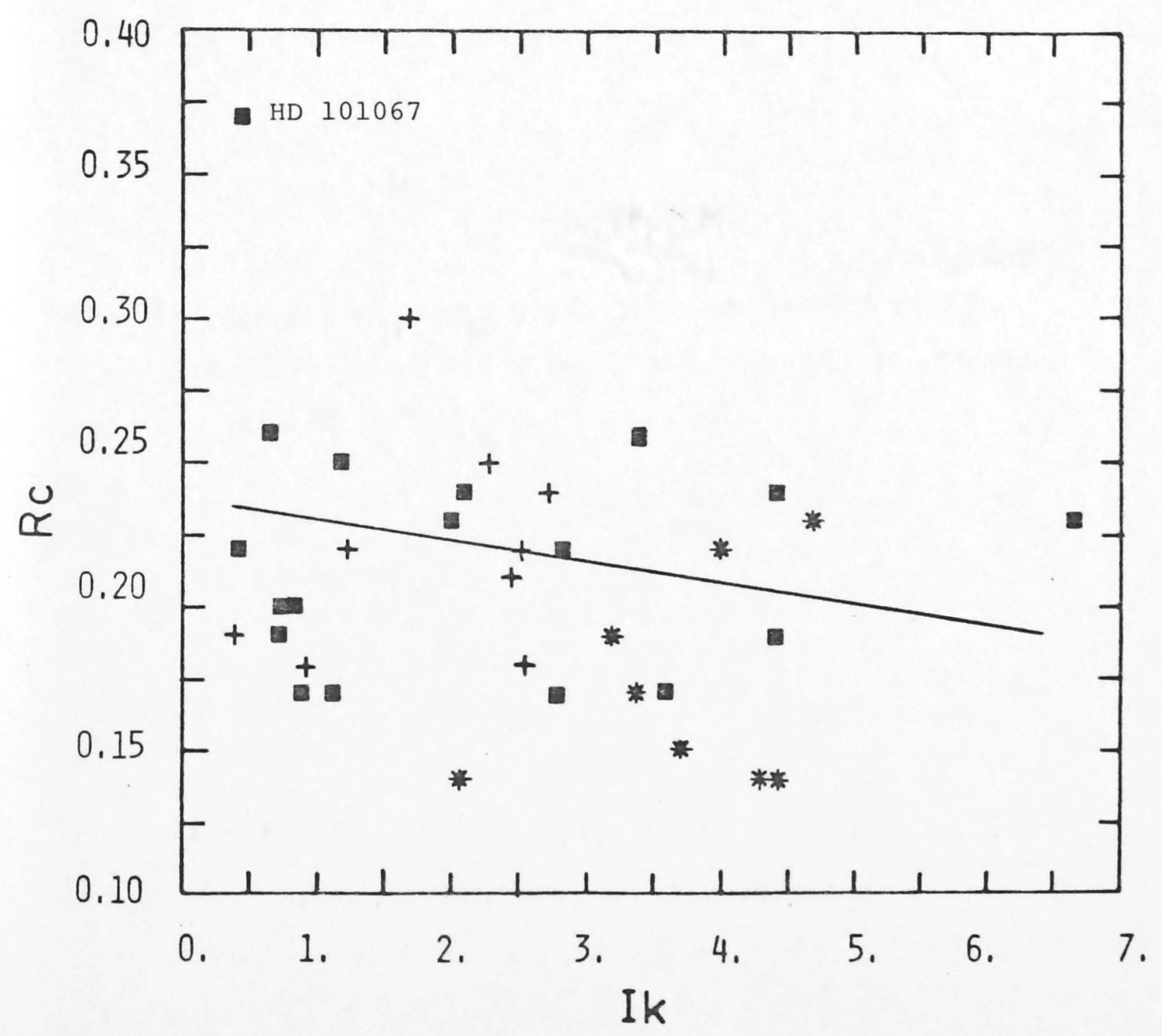


FIGURE $3.3 \mathrm{C}$

Rc as a function of Ik for supergiants. Stars are binned according to $G(+), K\left({ }^{2}\right)$ and $M\left({ }^{*}\right)$ spectral types. 


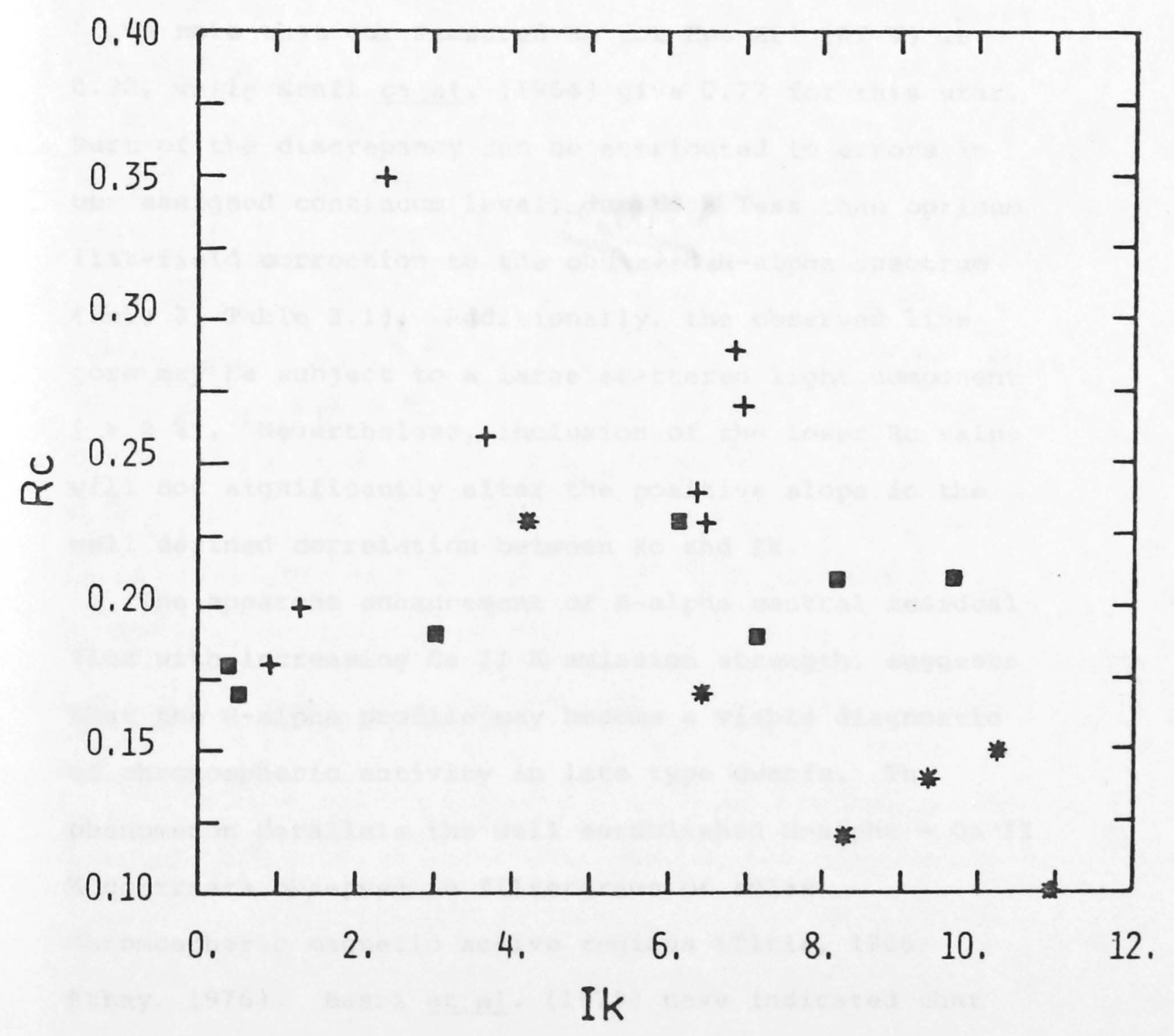




\section{(a) The Dwarfs}

The $G$ and $K$ dwarfs separately show a brightening of the H-alpha residual core flux (enhanced RC) with increasing $\mathrm{Ca}$ II $\mathrm{K}$ emission strength. The scatter at low values of Ik in Figure 3.3a is, in part, related to greater inaccuracies in the location of the $\mathrm{Kl}$ minima for stars displaying apparently weak $\mathrm{K}$ emission (e.g. Delta Pav $[\mathrm{G} 8 \mathrm{~V}])$.

We note that our measured Rc for Eps Eri (K2 V) is 0.30, while Kraft et al. (1964) give 0.27 for this star. Part of the discrepancy can be attributed to errors in our assigned continuum level, due to a less than optimum flat-field correction to the observed H-alpha spectrum (note 3, Table 3.1). Additionally, the observed line core may be subject to a large scattered light component ( $>2 \%)$. Nevertheless, inclusion of the lower Rc value will not significantly alter the positive slope in the well defined correlation between Rc and Ik.

The apparent enhancement of $\mathrm{H}$-alpha central residual flux with increasing $C a$ II $K$ emission strength, suggests that the H-alpha profile may become a viable diagnostic of chromospheric activity in late type dwarfs. The phenomenon parallels the well established H-alpha - Ca II $\mathrm{K}$ contrasts observed in filtergrams of solar chromospheric magnetic active regions (Title, 1966; Athay, 1976). Basri et al. (1979) have indicated that the latter contrasts can be understood in terms of varying chromospheric temperature distributions between 
quiet and active solar regions, and the resulting

differences in densities and optical depth scales.

Consequently, the correlation in Figure 3.3 a may reflect differing chromospheric thermodynamic conditions in the active dwarf stars, perhaps related to the incidence of active regions on their surfaces similar to solar plages. We return to a more detailed investigation of this point in Chapter Five.

An interesting feature of the strong $\mathrm{Ca}$ II $\mathrm{K}$ emission line dwarfs is the peculiar perturbations in $\mathrm{H}-a l$ pha core shape. Stars with relatively weak $\mathrm{K}$ emission (e.g. Beta Hyi, Delta Pav) exhibit well defined "U-shaped" H-alpha cores. On the other hand, many of the strong line dwarfs (e.g. Eps Eri, 70 Oph A, 83 Leo) show narrow, peaked H-alpha cores. Closely spaced, unresolved chromospheric emission components in the H-alpha cores of the latter stars could be responsible for this anomaly. Examination of the H-alpha core profile of the active star Xi Boo A reveals the presence of two possibly resolved emission structures about line centre.

\section{(b) Giants And Supergiants}

The giants and supergiants display considerably more scatter in the Rc-Ik plane than the dwarfs. However, there is a weak tendency for deeper H-alpha cores to be associated with stronger $\mathrm{K}$ emission among the giants. Further investigation is required for confirmation of this trend, although it is interesting that the effect is opposite to that revealed by the dwarfs. We re-discuss 
this difference in Chapter Six.

Finally, we note a temperature effect in the sense that cooler stars tend to have smaller Rc than their hotter counterparts at a given value of Ik. We caution that since Ik is temperature dependent, then comparisons between stars of differing spectral types may be misleading. In view of the large scatter in Figures $3.3 \mathrm{~b}$ and $c$, we conclude that the H-alpha central residual flux is an indeterminate chromospheric activity index for low gravity stars.

\subsection{COMPARISON OF H-ALPHA AND $\mathrm{Ca}$ II $\mathrm{K}$ LINE WIDTHS}

\subsubsection{The Empirical Ho-Wo Relation}

Figure 3.4 depicts the distribution of Log(Ho) against Log(Wo) (or equivalently, Mv) for the atlas stars with detected Ca II K emission (note 5, Table 3.1). Uncorrected widths are plotted in this diagram. It is clear that the H-alpha full width at half depth does not depend tightly on the W-B width, but a dependence albeit nonlinear and scattered - is suggested nonetheless (the solid line in this figure represents a semi-empirical fit to the data, which is discussed in more detail in section 3.4 .2$)$.

The following features are also evident:

(i) the H-alpha width shows a spectral type dependence in that cooler stars (e.g. M type) tend to have smaller Ho at a given Wo. Kraft et al. (1964) reported a similar behaviour and indicated that the effect could be 


\section{FIGURE 3.4}

Log(Ho) versus $\log ($ Wo $)$ for stars grouped according to G $(+), K($ ) and $M(*)$ spectral types. Note the widespread scatter in Ho for $\mathrm{G}$ and $\mathrm{K}$ giants/supergiants in the domain Log $($ Wo $) \geqslant 1.95$. The solid line represents a semi-empirical fit to the data by Fosbury (1973). Further details are given in the text. 


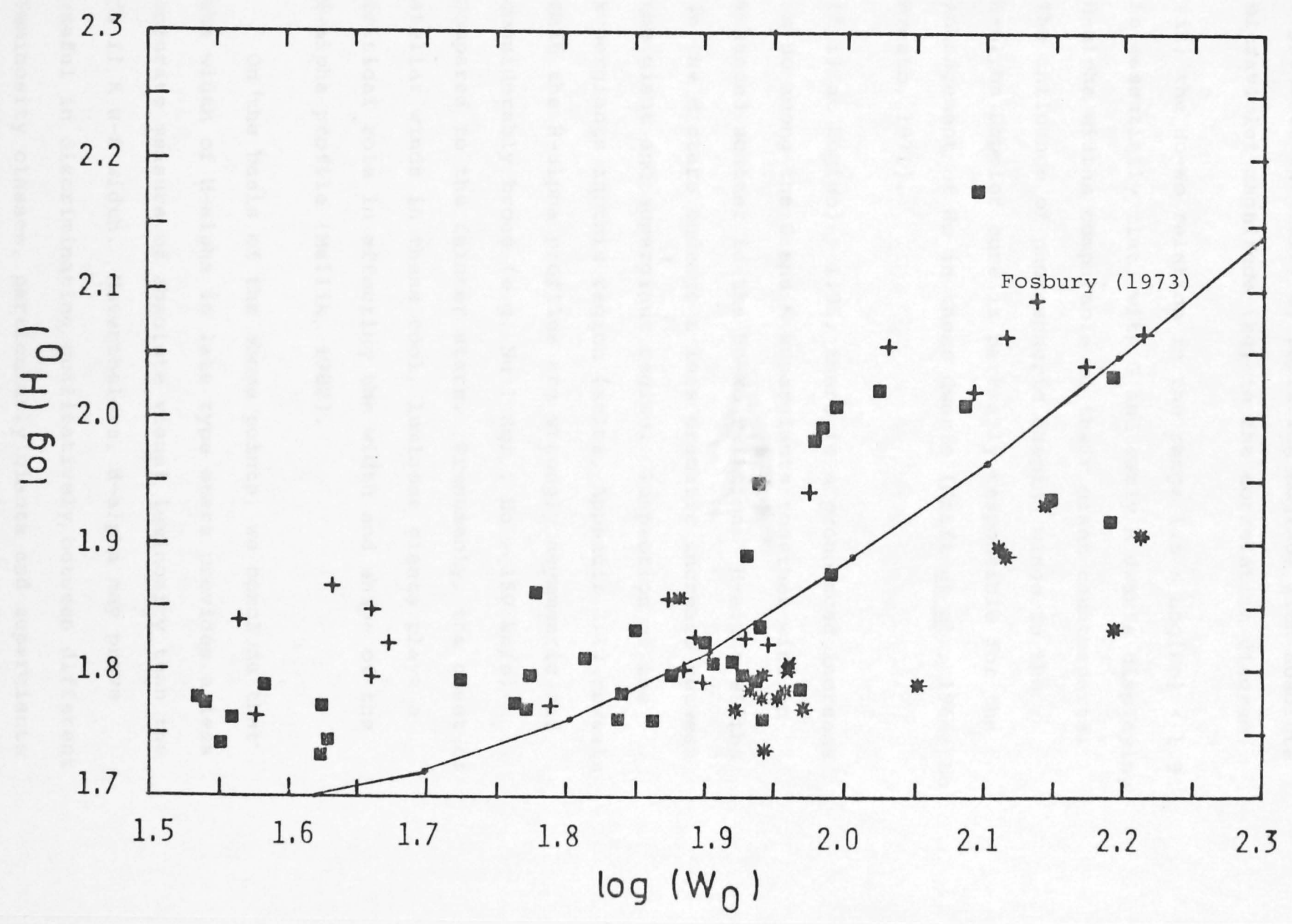


suppressed partially by replacing Log(Wo) with absolute ultraviolet magnitude (Mu) in the correlation diagram.

(ii) the Ho-Wo relation in the range $1.5<\log ($ Wo $)<1.9$ is essentially flat, with $G$ and early $K$ dwarfs displaying H-alpha widths comparable to their giant counterparts. The influence of photospheric damping wings in the H-alpha Doppler core is partially responsible for the enhancement of Ho in these dwarfs (Kraft et al., 1964; Lo Presto, 1971).

(iii) at $\log ($ Wo $)>1.95$, there is a pronounced increase in Ho among the $G$ and $K$ supergiants together with an enhanced scatter in the Ho-Wo relation. H-alpha widths in the $M$ stars undergo a less dramatic increase between the giant and supergiant regime. Inspection of the supergiants in this region (atlas, Appendix 2.1) reveals that the H-alpha profiles are strongly asymmetric and considerably broad (e.g. Nu $1 \mathrm{Sgr}$, Ho $\sim 150 \mathrm{~km} / \mathrm{s}$ ) compared to the fainter stars. Presumably, the onset of stellar winds in these cool, luminous giants plays a critical role in affecting the width and shape of the H-alpha profile (Mallik, 1982).

On the basis of the above points, we conclude that the width of H-alpha in late type stars provides a less accurate measure of absolute visual luminosity than the Ca II K W-B width. Nevertheless, H-alpha may prove useful in discriminating, qualitatively, between different luminosity classes, particularly giants and supergiants 
(cf. Imhoff, 1977). Furthermore, Fosbury (1973) has demonstrated that a coordinated study of Ho and wo can reveal insight into the extent to which chromospheric Doppler motions affect the width-luminosity (W-L) broadening of $\mathrm{H}-\mathrm{al}$ pha and $\mathrm{Ca}$ II K features. His analysis, together with a variation on its approach, is discussed in the following section.

\subsubsection{The Role of Chromospheric Microturbulence In}

H-alpha And $\mathrm{Ca}$ II K Width-Luminosity Correlations.

In the idealised case of a finite slab forming region with constant source function and height independent Doppler width $\left(\Delta \lambda_{D}\right)$, the FWHM (wo) of an optically thick Doppler broadened line can be expressed in velocity units (Goldberg, 1957) as,

$$
\text { Wo } \simeq 2 \Delta \lambda_{D}\left(\operatorname{Ln} \tau_{0}+0.4\right)^{1 / 2} \quad \tau_{0}>1 \quad(3.2)
$$

where $\tau_{0}$ is the line centre optical thickness of the slab and,

$$
\Delta \lambda_{D}=\left\{\frac{2 k T}{M_{A}}+\text { vturb }^{2}\right\}^{1 / 2} \quad[\mathrm{~km} / \mathrm{s}]
$$

where $\mathrm{M}_{\mathrm{A}}$ denotes the atomic mass and Vturb the non-thermal broadening velocity (specifically, microturbulence).

Equation 3.2 indicates that the width of a Doppler controlled line can provide information on the velocity distribution (thermal and non-thermal) of atoms involved in a particular transition. However, in view of the approximations implicit in its derivation, care must be 
exercised in its practical application to observed spectral lines.

On the assumption that Ho and Wo are Doppler broadened, Fosbury (1973) has shown that the slab model provides a useful paradigm to study the composite $W-L$ broadening of H-alpha and Ca II K features. Briefly, his analysis proceeds as follows.

For the case of H-alpha, equation 3.2 can be written as,

$$
\text { Ho } \simeq 4 \Delta \lambda_{D}{ }^{H \alpha} \simeq 4\left(100+\text { Vturb }{ }^{2}\right)^{1 / 2} \quad \tau_{H \alpha}=50
$$

where a mean chromospheric optical depth of 50 and a kinetic electron temperature of $6000 \mathrm{~K}$ (as representative of the H-alpha line formation region in the chromosphere) have been assumed. The latter temperature corresponds to a mean hydrogen thermal broadening of $10 \mathrm{~km} / \mathrm{s}$.

For Ca II K, equation 3.2 assumes the form

$$
\text { Wo } \simeq 6 \Delta \lambda_{D}^{K} \simeq 6 \text { Vturb } \quad \tau_{K}=10^{4}
$$

where a chromospheric ${ }^{\tau_{K}}$ of $1.0 \mathrm{E} 4$ has been adopted (cf. solar K line thermalisation depth; Linsky and Avrett, 1970). In addition, the thermal contribution to the $\mathrm{K}$ line Doppler width has been neglected owing to the forty times larger calcium atomic weight compared to hydrogen. To illustrate the usefulness of the above expressions, we consider their application to the H-alpha and $\mathrm{Ca}$ II $\mathrm{K}$ profiles of Arcturus. Using $\mathrm{HO}=63 \mathrm{~km} / \mathrm{s}$ (corrected from Table 3.1 ), equation 3.3 implies Vturb $13 \mathrm{~km} / \mathrm{s}$. This value is a reasonable upper limit to the 
2-10 km/s variation derived independently from model profile fits to the Arcturus Ca II and Mg II resonance lines (Ayres and Linsky, 1975b). For a corrected W-B width of $77 \mathrm{~km} / \mathrm{s}$ (Table 3.1 ), equation 3.4 predicts Vturb= $12 \mathrm{~km} / \mathrm{s}$ which is comparable to that inferred from Ho. Hence, despite its inherent approximations, the slab model may be useful in the derivation of upper limits to the adhoc microturbulence parameter, provided one remains within the Doppler core of the line profile.

Studies of the solar chromosphere have revealed a correlation between $\mathrm{H}-\mathrm{al}$ pha absorption and $\mathrm{Ca}$ II $\mathrm{K}$ emission structures (McMath et al., 1956). This result indicates that these two spectral features are likely to be formed in overlapping chromospheric regions and, consequently, are affected by the same non-thermal broadening velocities. If a similar situation holds in non-solar stars, then the quantity Vturb can be eliminated from equations 3.3 and 3.4 to yield a formula relating Ho and wo,

$$
\mathrm{HO} \simeq 4\left\{100+\frac{\text { Wo }^{2}}{36}\right\} \quad 1 / 2
$$

Using ${ }^{\tau} \mathrm{H} \alpha$ as a free parameter in this equation, Fosbury (1973) has generated a family of curves in the Log(Ho)-Log(Wo) plane. A good fit to the $K, G$ and late $F$ stars was found for $\tau_{\mathrm{H} \alpha}=50$. For comparison with our data, equation 3.5 (corrected for instrumental effects) is plotted in Figure 3.4. The relation accounts approximately for the non-linearity in the diagram. The $G$ and early K dwarfs are deviant due to the presence of 
$\mathrm{H}$-alpha damping wings, while $\mathrm{K}$ supergiants show a widespread scatter.

Rather than pursue this treatment further, we propose an alternative approach to the examination of Ho and Wo. As reviewed in Chapter one, there is still considerable debate as to whether the $\mathrm{W}-\mathrm{B}$ width is Doppler controlled - and thus, responding primarily to the systematic increase in non-thermal velocities with increasing stellar luminosity - or Damping controlled and, thus, opacity broadened as a consequence of the chromospheric "thickening" in low gravity stars (cf. Ayres, 1979). It appears (Linsky, 1980) that the key parameter underlying these two broadening theories is the local $\mathrm{K}$ line Doppler width, with $3 \Delta \lambda_{\mathrm{D}}$ representing the canonical demarkation point (Mihalas, 1978) separating profile widths from being formed within the Doppler core $\left(W_{0} / 2<\right.$ $\left.3 \Delta \lambda_{D}\right)$ and in the Lorentzian line damping wing ( Wo/2>3 $\left.>\lambda_{D}\right)$.

In the case of $\mathrm{H}$-alpha, the much lower chromospheric opacity in the line (compared to Ca II K), likely ensures that the H-alpha width is confined to the Doppler core of the line absorption coefficient (except, possibly, in dwarfs with extensive damping wings). Thus, equation 3.3 can be utilised to estimate approximately vturb (or equivalently, $\Delta \lambda_{D}$ ) from Ho. These non-thermal velocities can be then compared directly with corresponding values of Wo. Accordingly, an interesting and, perhaps, more meaningful analysis of Ho and Wo widths is to consider the data mapped in the Wo/ $\Delta \lambda_{D}$ - Wo plane, as shown in 
Figure 3.5 (note that instrumentally corrected widths are used in the generation of this figure).

Evidently, many of the giants in the range $65<$ wo < $95 \mathrm{~km} / \mathrm{s}$ lie above the Wo $=6 \Delta \lambda_{D} \mid$ boundary line (i.e. Wo $/ 2$ $\left.>3 \Delta \lambda_{\mathrm{D}}\right)$. Since the influence of the Doppler dominated portion of the line absorption profile for the strong $\mathrm{Ca}$ II $K$ transition does not extend beyond about $3 \Delta \lambda_{D}$, our result suggests that wo for this group of stars is formed in the $\mathrm{K}$ line damping wing and, accordingly, is weakly sensitive to non-thermal velocities.

The interpretation of Figure 3.5 is reliant upon several considerations :

(1) A value of $\tau \mathrm{H} \alpha=50$ has been adopted in the evaluation of Vturb from Ho. Fosbury (1973) has indicated that this estimate is optimum for many late type stars, but may vary somewhat between spectral types, and is likely to be larger in extended chromospheres of low gravity stars. For a given Ho and wo, equation 3.2 implies that a reduction in ${ }^{\tau} \mathrm{H} \alpha$ would correspond to an increase in vturb and, consequently, a decrease in Wo/ $/ \Delta \lambda_{D}$. However, since $\tau_{H} \alpha$ appears as the argument of a logarithm, large changes would correspond to only small perturbations in vturb.

(2) The $\mathrm{G}$ and $\mathrm{K}$ dwarfs at Wo $<45 \mathrm{~km} / \mathrm{s}$ all lie below the Wo $=6 \Delta \lambda_{D}$ line, suggesting Doppler dominated Wo. However, we note that the H-alpha profiles of these stars display pronounced damping wings which contribute to Ho and, subsequently, lead to overestimated Vturb. 


\section{FIGURE 3.5}

The ratio Wo $/ \Delta \lambda_{D}$ is plotted against Wo for stars grouped according to $G(+), K($ a $)$ and $M(*)$ spectral types. $\Delta \lambda_{D}$ denotes the mean upper limit Ca II K Doppler width inferred from Ho measurements (eq. 3.5$)$. The ratio Wo/ $\Delta \lambda_{D}$ measures approximately the distance from line centre (in $1 / 2$ Doppler width units) of the intensity point at which wo is estimated. The horizontal solid line corresponds to the Wo/2 $=3 \Delta \lambda_{D}$ boundary limit separating the Doppler core from the damping wing of the $\mathrm{K}$ line absorption profile. In the text, we argue that wo for stars lying above this line is primarily opacity broadened. 


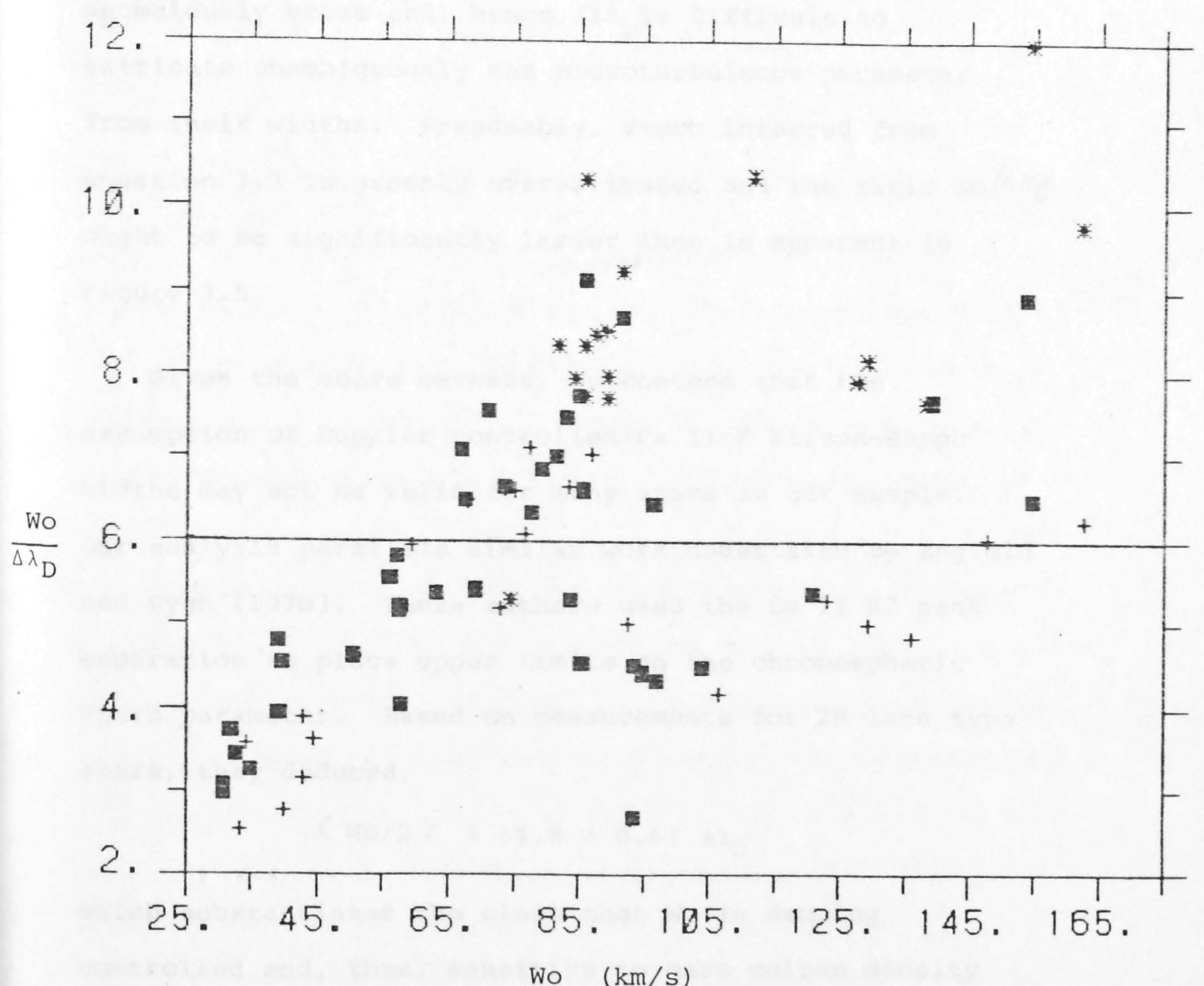


(3) Several giants and supergiants in the domain wo > 80 $\mathrm{km} / \mathrm{s}$ also lie below the $6 \Delta \lambda \mathrm{D}$ boundary line. These stars exhibit marked $\mathrm{H}-\mathrm{al}$ pha and $\mathrm{Ca}$ II $\mathrm{K}$ profile asymmetries indicative of macroscopic chromospheric motions. Under these circumstances, the constant properties slab model is an inadequate representation for the H-alpha line formation region. The observed H-alpha profiles are anomalously broad and, hence, it is difficult to extricate unambiguously the microturbulence parameter from their widths. Presumably, Vturb inferred from equation 3.3 is grossly overestimated and the ratio wo $/ \Delta \lambda_{D}$ ought to be significantly larger than is apparent in Figure 3.5 .

Given the above caveats, we contend that the assumption of Doppler controlled Ca II K Wilson-Bappu widths may not be valid for many stars in our sample. Our analysis parallels similar work undertaken by Engvold and Rygh (1978). These authors used the Ca II K2 peak separation to place upper limits on the chromospheric Vturb parameter. Based on measurements for 26 late type stars, they deduced,

$$
\left\langle W_{0} / 2\right\rangle>(4.8 \pm 0.6) \Delta \lambda_{D}
$$

which substantiates the claim that wo is damping controlled and, thus, sensitive to mass column density effects (Ayres, 1979).

It is important to recognise that the $\mathrm{W}-\mathrm{L}$ broadening of $\mathrm{H}$-alpha indicates an increase in Doppler motions in low gravity stars. Nevertheless, such motions may be 
still involved in the broadening of optically thick Ca II K features by, for example, the effects of Doppler redistribution in low density giants (Basri, 1979; 1980). Thus, Doppler and Damping scenarios may not be mutually exclusive interpretations of the Wilson-Bappu effect. However, our preliminary analysis indicates that the H-alpha profile width can provide important constraints on the extent to which Doppler non-thermal motions influence $\mathrm{Ca}$ II $\mathrm{K}$ W-B widths.

\subsection{COMPARISON OF H-ALPHA AND CA II K PROFILE}

\section{ASYMMETRIES}

In this concluding section we examine the $\mathrm{H}$-alpha absorption and $\mathrm{Ca}$ II $\mathrm{K}$ emission profile shapes, concentrating on the extent to which large-scale velocity induced asymmetries are reflected in their chromospheric cores.

Stencel (1978) has discovered a trend of Ca II K blue asymmetry $(\mathrm{Fv} / \mathrm{Fr}>1)$ for giant stars hotter than $\mathrm{K} 3$ and red asymmetry $(\mathrm{Fv} / \mathrm{Fr}<1)$ for their counterparts cooler than K4. Studies of $\mathrm{Ca}$ II $\mathrm{K}$ in the solar chromosphere have indicated that upward propagating acoustic (Heasley, 1975; Cram, 1976) or gravity (Cram et al., 1977; Cram, 1978 ) waves may be important dynamical processes underlying observed $\mathrm{Ca}$ II $\mathrm{K}$ blue asymmetry. The shift to red asymmetry has been attributed to the onset of systematic outward gas flows in the chromospheres of the cooler giants (Mullan, 1978).

Figure 3.6 depicts this dichotomy for the Ca II K 


\section{FIGURE 3.6}

Program stars are plotted in the $M v,(B-V)$ plane. Crosses denote stars with $\mathrm{Fv} / \mathrm{Fr}>0.9$. Filled squares represent stars with $\mathrm{Fv} / \mathrm{Fr}<0.9$. Stars displaying significant absorption at $\mathrm{K}_{1 \mathrm{~V}}$ and, hence, uncertain $\mathrm{W}_{0}$ are omitted. A schematised colour-luminosity array of $M 67$ is shown for reference. 


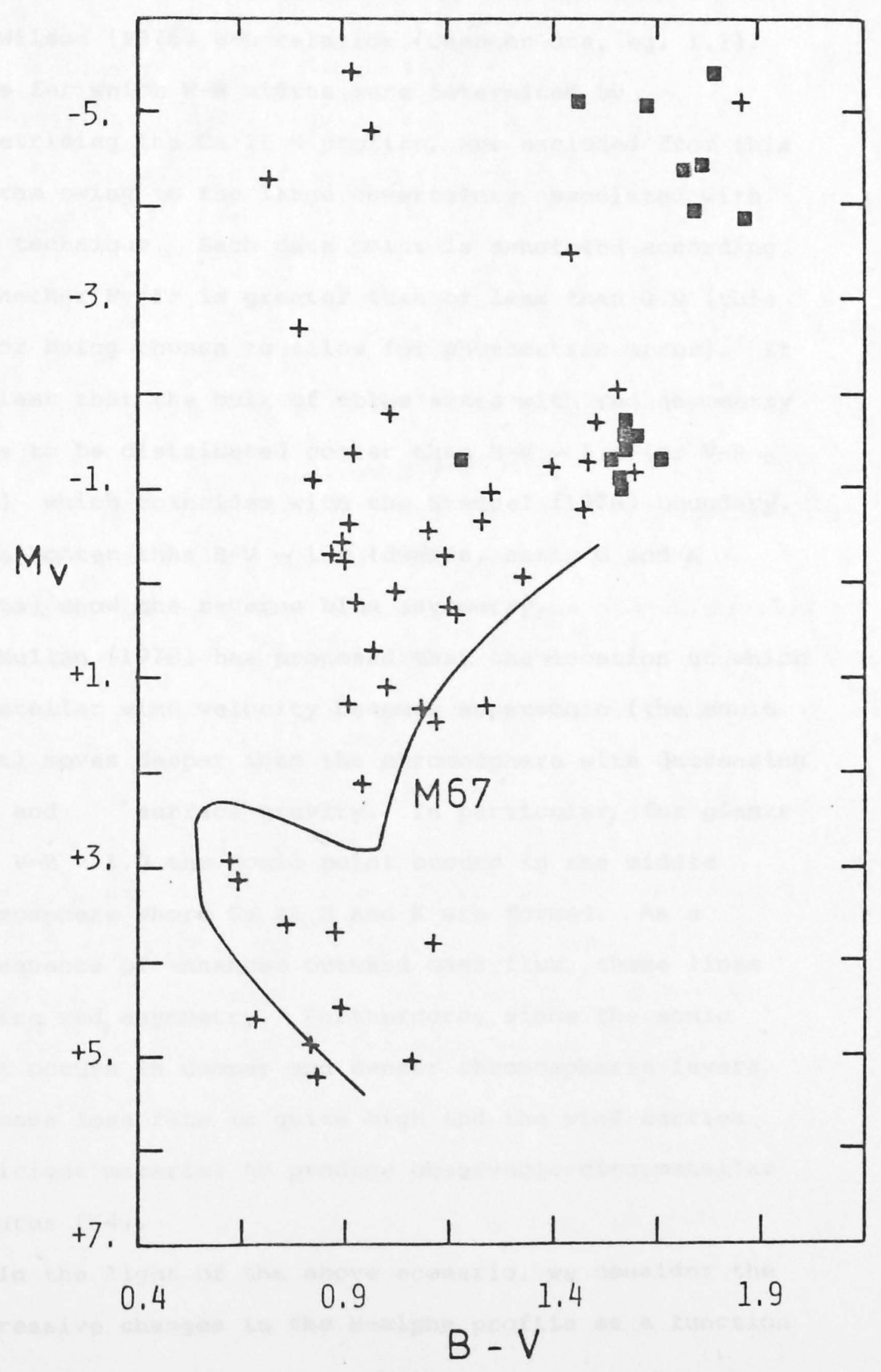


profiles exhibited in Chapter Two. The distribution of programme stars is mapped in the $\mathrm{Mv}-(\mathrm{B}-\mathrm{V})$ plane using absolute visual magnitudes derived from corrected wo in the Wilson (1976) W-L relation (Chapter one, eq. 1.1). Stars for which $W-B$ widths were determined by symmetrising the Ca II K profile, are excluded from this diagram owing to the large uncertainty associated with this technique. Each data point is annotated according to whether Fv/Fr is greater than or less than 0.9 (this factor being chosen to allow for photometric error). It is clear that the bulk of atlas stars with red asymmetry tends to be distributed cooler than $\mathrm{B}-\mathrm{V} \sim 1.4$ (or $\mathrm{V}-\mathrm{R} \sim$ 1.06) which coincides with the stencel (1978) boundary. Stars hotter than $B-V \sim 1.4$ (dwarfs, early $G$ and $K$ giants) show the reverse blue asymmetry.

Mullan (1978) has proposed that the location at which the stellar wind velocity becomes supersonic (the sonic point) moves deeper into the chromosphere with decreasing Teff and surface gravity. In particular, for giants with $V-R>1.0$ the sonic point occurs in the middle chromosphere where $\mathrm{Ca}$ II $\mathrm{H}$ and $\mathrm{K}$ are formed. As a consequence of enhanced outward mass flux, these lines acquire red asymmetry. Furthermore, since the sonic point occurs in deeper and denser chromospheric layers, the mass loss rate is quite high and the wind carries sufficient material to produce observable circumstellar features (K4).

In the light of the above scenario, we consider the progressive changes in the $\mathrm{H}$-alpha profile as a function 
of the shift in Ca II K asymmetry across the Stencel

(1978) wind boundary. Examination of the atlas reveals the following features :

(i) Giants hotter than G8, which display blue Ca II K asymmetry (e.g. Delta Lep, Mu Vel, Beta Crv and Delta Col), generally exhibit symmetric H-alpha profiles.

(ii) Giants slightly to the left of $B-V \sim 1.4$ (e.g. Pi Hya, Beta Ceti, Arcturus and Beta Col) on average show symmetric or slightly blue asymmetric Ca II K. However, the corresponding $\mathrm{H}$-alpha profiles demonstrate a perceptible asymmetry on the red side of their absorption cores and, possibly, a small core blue-shift. This relative behaviour indicates that the sonic point is located in the upper chromosphere - in the representative layers of H-alpha core formation - but, is not sufficiently deep to affect significantly the Ca II K2 emission.

(iii) Cooler giants to the right of the wind boundary (e.g. K stars; Sig Pup, N Vel, Eps Lep and M stars; Nu Vir, Delta Vir, Psi Vir) exhibit preponderant red Ca II $\mathrm{K}$ asymmetry. A red asymmetry and slight core blue-shift also persists in the corresponding H-alpha profile of these stars.

(iv) Cool supergiants display pronounced Ca II K red asymmetry which, in many cases, is accentuated by the presence of blue-shifted K4 features (see, for example, HD93070, Nu l Sgr, HD68553, HD89388 and HD63032). The 
corresponding $\mathrm{H}-\mathrm{al}$ pha profiles exhibit marked core asymmetries and enhanced widths compared to giants of similar spectral type. Interestingly, stars with significant Ca II K4 absorption do not appear to reflect circumstellar effects in their corresponding $\mathrm{H}-a l p h a$ profiles.

Furthermore, in many of the cool supergiants there are clear indications that the velocity fields which determine the H-alpha core asymmetry map directly into the breadth of the Ca II K feature (see, for example, Beta Ara, Lambda Vel and Sigma Cma). This apparent correlation is most dramatic in the G supergiants HD 57146 and HD 59890 where fine structures present in H-alpha appear to be reflected faithfully in the central Ca II K3 absorption component(s). Generally, the mapping occurs with an increase of scale and a blue-shifting of K3 with respect to the flux minimum in the H-alpha core. We conclude from this behaviour that for a mass conservative gas flow (Chiu et al., 1977), the K3 absorption feature is formed above the representative layers of $\mathrm{H}$-alpha core formation.

The above qualitative comparison of $\mathrm{H}-\mathrm{alpha}$ and $\mathrm{Ca}$ II $\mathrm{K}$ profiles highlights dramatically the important role played by systematic chromospheric velocities in dictating the behaviours of strong spectral lines. Moreover, the correspondence between $\mathrm{H}-\mathrm{al}$ pha and $\mathrm{K} 3$ absorption should prove ultimately to be a valuable diagnostic of chromospheric mass motions in cool giants 
and supergiants. 


\subsection{INTRODUCTION}

This chapter describes the computer code used for the synthesis of $\mathrm{H}-\mathrm{alpha}$ and $\mathrm{Ca}$ II $\mathrm{K}$ profiles from prototype distributions of chromospheric temperature and microturbulence. The Complete Linearisation Method of Auer and Mihalas (1969), modified to incorporate hydrostatic equilibrium (HSE) adjustment of electron and hydrogen number densities in a fixed temperature atmosphere, is employed in the solution of the radiative transfer equation. Implicit in the code are the assumptions of a static, plane-parallel, homogenous atmosphere and scattering with complete redistribution (CRD) .

In the following sections we describe the mathematical basis of the profile synthesis code (as implemented on the A.N.U Univac 1100$)$, outlining the sequence of calculations that lead to the derivation of radiation fields consistent with non-LTE hydrogen (and calcium) energy level populations. We commence with a brief description of the model atmosphere and model atoms which underlie the transfer solution. 


\subsection{MODEL ATMOSPHERE}

In the absence of radiative equilibrium ( $R E$ ) the chromospheric (and upper photospheric) temperature distribution is difficult to compute owing to uncertainties in the nature of the non-radiative heating mechanisms that maintain the chromospheric temperature rise. Excellent reviews on the topic of chromospheric heating are provided by Athay (1976) and Ulmschneider (1979).

The approach adopted in this thesis for the construction of a model atmosphere grid is equivalent to that applied to studies of $\mathrm{Ca}$. I and $\mathrm{Mg}$ II line formation in the Stellar Model Chromospheres series (Linsky, 1980, and references therein). In summary, the method involves:

(1) use of mass column density ( $\mathrm{m}, \mathrm{gm} \mathrm{cm}-2$ ) as the basic height parameter. This choice of variable leads to considerable simplification in the HSE calculation of number densities (described in sections 4.4 and 4.8 .3 ).

(2) modelling of the chromospheric thermal structure by an adhoc temperature rise, linear in $\log (\mathrm{m})$, adjoined to an $\mathrm{RE}$ photospheric model at a pre-determined temperature minimum (Tmin). Usually, 20 mass points are derived from a published photosphere model which is characterised by an effective temperature (Teff), a metallicity [Fe/H] and a surface gravity $(g)$. The deepest photospheric point is 
chosen to ensure thermalisation of transition source functions to the Planck function, $B v(T)$, such that the LTE lower boundary condition is satisfied (section 4.6). Typically, $4 \varnothing$ equispaced points in $\log (\mathrm{m})$ are assigned to the region of the chromospheric temperature rise.

(3) simulation of the coronal transition zone (TZ) by a sharp temperature increase at the "top" of the model chromosphere. The latter point is defined to occur at optical depth unity at the head of the opaque Lyman continuum. A steep temperature rise above $8000-9000 \mathrm{~K}$ is expected because the Lyman continuum is no longer an efficient radiator above these temperatures (Thomas and Athay, 1961; Athay, 1981). Generally, 10 grid points are employed in specifying the $\mathrm{TZ}$ with the uppermost mass point selected to give an optical depth of $\emptyset . \emptyset 1$ in the Lyman continuum, at a temperature of $20,000 \mathrm{~K}$.

(4) inclusion of an isotropic microturbulence, V(m), which is constant in the photosphere, and increasing linearly with $\log (\mathrm{m})$ through the chromosphere.

\subsection{MODEL ATOMS}

\section{(a) Hydrogen}

In this study we consider an idealised hydrogen atom consisting of 5 bound states plus a continuum level. The level statistical weights and corresponding energies above the ground state for this model atom are provided in Table 4.1. 
TABLE 4.1

ATOMIC PARAMETERS FOR THE MODEL HYDROGEN ATOM

\begin{tabular}{cccc}
\hline Level & Designation & $\begin{array}{c}\text { Energy } \\
\lambda_{i}\left(\mathrm{~cm}^{-1}\right)\end{array}$ & $\begin{array}{c}\text { Statistical Wt. } \\
g_{i}\end{array}$ \\
\hline 1 & $1 \mathrm{~s}$ & 0.0 & 2 \\
2 & $2 \mathrm{~s}$ & 82259.07 & 8 \\
3 & $3 \mathrm{~s}$ & 97492.23 & 18 \\
4 & $4 \mathrm{~s}$ & 102823.84 & 32 \\
5 & $5 \mathrm{~s}$ & 105291.61 & 50 \\
6 & continuum & 109678.75 & - \\
\hline
\end{tabular}


The radiative transitions specifically treated in

the transfer solution comprise the optically thick Lyman continuum, H-alpha, Ly-alpha and Ly-beta. Detailed

balance is not assumed for the latter resonance lines as previous calculations have demonstrated that small departures from this approximation can, for example, lead to significant changes in the computed hydrogen ionisation balance in the middle solar chromosphere (Vernazza et al., 1973).

In Table 4.2 we list, for each transition ( $i$ to $j$ ), the central wavelength, oscillator strength (fij) (Green et al., 1957) and radiative damping width (Wiese et al., 1969). In addition to the explicit transitions, we incorporate various optically thin interactions between the upper hydrogen levels (in particular, the Balmer and Paschen continua) in terms of pre-specified fixed rates. These will be more fully discussed in section 4.7 .1 .

\section{(b) Calcium}

The Ca I-Ca II ion is likewise represented by a 5 levels plus continuum model atom (as depicted in figure 4.1), with zero energy corresponding to the first ionised state (4s). The pertinent energy level parameters for this schematic atom are presented in Table 4.3. We solve for the $\mathrm{H}$ and $\mathrm{K}$ resonance doublet and infra-red (IR) triplet transitions between the $4 \mathrm{p}$ and $3 \mathrm{~d}$ states. The latter lines are included as they provide an important means of indirect coupling between the $4 \mathrm{P} 1 / 2$ and $4 \mathrm{P} 3 / 2$ levels, and thereby affecting the $H$ and $K$ line 


\section{TABLE 4.2}

TRANSITION PARAMETERS FOR HYDROGEN LINES

Parameter

$1-2(\operatorname{Ly} \alpha) \quad 1-3(\operatorname{Ly} \beta) \quad 2-3(\mathrm{H} \alpha)$

$\lambda_{i j}(\AA)$

1215.67

1025.72

6562.80

$f_{i j}$

0.416

0.0791

0.6407

$\Gamma_{\text {rad }}\left(\sec ^{-1}\right)$

$4.69(8)$

$0.997(7)$

$5.69(7)$

$\lambda_{i j}$ : central wavelength

$f_{i j}$ : oscillator strength

$\Gamma_{\text {rad }}$ : radiative damping width 
FIGURE 4.1

Energy level diagram for the Ca I-Ca II model ion, showing the transitions treated in the radiative transfer calculation. 


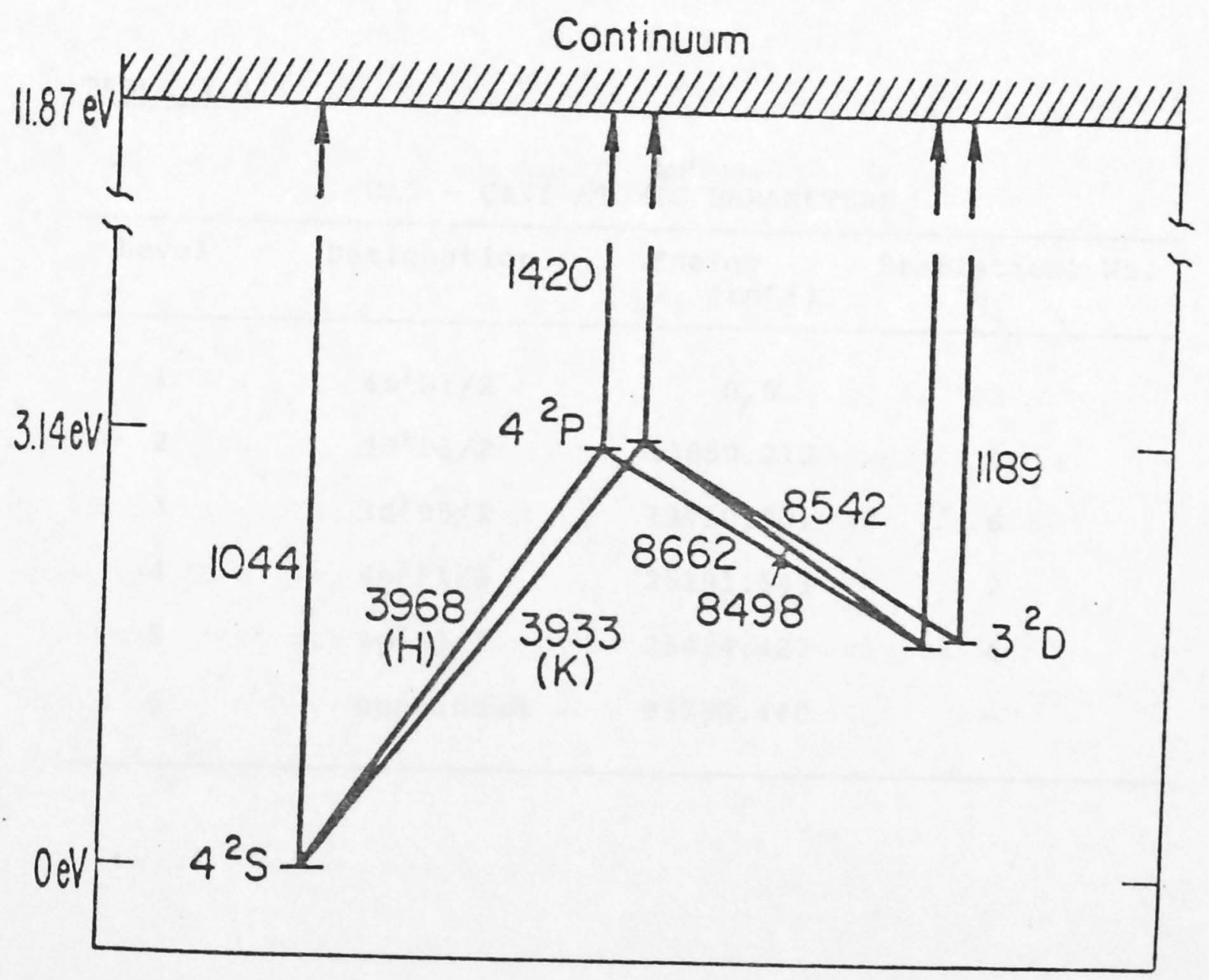


TABLE 4.3

\section{CaI - CaII ATOMIC PARAMETERS}

\begin{tabular}{cccc}
\hline Level & Designation & $\begin{array}{c}\text { Energy } \\
\lambda_{i}\left(\mathrm{~cm}^{-1}\right)\end{array}$ & $\begin{array}{c}\text { Statistical wt. } \\
g_{i}\end{array}$ \\
\hline 1 & $4 \mathrm{~s}^{2} \mathrm{~S} 1 / 2$ & 0.0 & 2 \\
2 & $3 \mathrm{~d}^{2} \mathrm{D} 3 / 2$ & 13650.212 & 4 \\
3 & $3 \mathrm{~d}^{2} \mathrm{D} 5 / 2$ & 13710.901 & 6 \\
4 & $4 \mathrm{p}^{2} \mathrm{P} 1 / 2$ & 25191.543 & 2 \\
5 & $4 \mathrm{p}^{2} \mathrm{P} 3 / 2$ & 25414.427 & 4 \\
6 & continuum & 95785.440 & - \\
\hline
\end{tabular}


transfer solutions (Linsky and Avrett, 197ø). Central wavelengths, oscillator strengths and radiative damping coefficients for the transitions are given in Table 4.4. The continua associated with the $4 \mathrm{~s}, 4 \mathrm{p}$ and $3 \mathrm{~d}$ states are treated as fixed rate transitions (section 4.7.1).

\subsection{CALCULATION OF LTE ATOMIC POPULATIONS}

LTE level populations $\left(\mathrm{Ni}^{*}\right)$ for the model atoms

presented in section 4.3 are important as starting solutions to the calculation of their non-LTE

counterparts from the coupled statistical equilibrium and radiative transfer equations. For the hydrogen atom, these quantities are derived from the Boltzmann equation,

$$
\frac{N i^{*}}{N h}=\frac{g_{i}}{U(T)} \exp (-\lambda i / k T)
$$

where $\mathrm{Nh}$ is the total hydrogen number density (neutrals plus protons), and $U(T)$ the partition function (asterisks denote LTE values).

The evaluation of $\mathrm{Nh}$ proceeds from the equations describing the hydrogen LTE ionisation balance. These are as follows:

(1) Momentum conservation

$$
\mathrm{Pg}+1 / 2 \rho \mathrm{V}^{2}=\mathrm{gm}
$$

(2) Mass conservation

$$
\rho=N h m_{H}\left(\sum_{i} A_{i} \mu_{i}\right)+N e m_{e}
$$

where $A_{i}$ and $\mu_{i}$ denote, respectively, abundances 
TABLE 4.4

TRANSITION PARAMETERS FOR CALCIUM LINES

\begin{tabular}{lccccc} 
Parameter & $1-5(\mathrm{~K})$ & $2-5$ & $3-5$ & $1-4(\mathrm{H})$ & $2-4$ \\
\hline$\lambda_{i j}(\AA)$ & 3933.644 & 8498.018 & 8542.089 & 3968.470 & 8662.14 \\
$\mathrm{f}_{\mathrm{ij}}$ & 0.66 & 0.00883 & 0.53 & 0.33 & 0.0442 \\
$\Gamma_{\mathrm{rad}}\left(\mathrm{sec}^{-1}\right)$ & $1.5(8)$ & $1.5(8)$ & $1.5(8)$ & $1.48(8)$ & $1.48(8)$ \\
\hline
\end{tabular}

$\lambda_{i j}:$ central wavelength

$f_{i j}$ : oscillator strength

$\Gamma_{\text {rad }}$ : radiative damping width 
(relative to hydrogen) and atomic weights of the atmospheric constituents listed in Table 4.5 .

(3) Perfect gas law

$$
\operatorname{Pg}=N h\left(\sum_{i} A_{i}\right) k T+N e k T
$$

(4) Charge conservation

$$
N e=N h \sum_{i} \frac{A_{i}}{1+N e \Psi_{i}(T)}
$$

where $\psi_{i}$ is the LTE Saha ionisation factor of each of the above elements.

The first relation arises from the assumption of HSE in a one dimensional atmosphere. Pg denotes the gas pressure, whilst the second term corresponds to turbulent pressure support. Equation 4.3 describes the net gas density $(p)$ due to electrons (Ne), atomic hydrogen and the atmospheric species listed in Table 4.5, whose abundances are appropriately scaled with respect to stellar metallicity. In equation 4.4 the gas pressure is deduced from the sum of partial pressures of the constituent elements and the electron pressure.

Expression 4.5 is obtained from combination of the Saha ionisation law with particle density conservation. It represents the net contribution to the electron density from ionisations of hydrogen and the elements in Table 4.5. Saha factors for the latter are computed using the ionic potentials and partition function values given by Vernazza et al., (1973). First ionisations of metals provide a large pool of electrons at temperatures 
TABLE 4.5

ATMOSPHERIC CONSTITUENTS EMPLOYED IN THE ELECTRON DENSITY CALCULATION

\begin{tabular}{|c|c|c|c|}
\hline Element & $\begin{array}{l}\text { Solar Abundance } \\
A_{i}(\text { relative to } H)\end{array}$ & $\begin{array}{c}\text { Atomic wt. } \\
\mu_{i}(a m u)\end{array}$ & $\begin{array}{c}\text { Ionic Pot. } \\
(\mathrm{ev})\end{array}$ \\
\hline $\begin{array}{l}\mathrm{H} \\
\mathrm{He} \\
\mathrm{C} \\
\mathrm{N} \\
\mathrm{O} \\
\mathrm{Ne} \\
\mathrm{Na} \\
\mathrm{Mg} \\
\mathrm{Al} \\
\mathrm{Si} \\
\mathrm{P} \\
\mathrm{S} \\
\mathrm{Ar} \\
\mathrm{K} \\
\mathrm{Ca} \\
\mathrm{Cr} \\
\mathrm{Mn} \\
\mathrm{Fe} \\
\mathrm{Co} \\
\mathrm{Ni}\end{array}$ & $\begin{array}{l}1.00 \\
1.00(-1) \\
5.37(-4) \\
1.14(-4) \\
6.76(-4) \\
3.54(-5) \\
2.81(-6) \\
4.46(-5) \\
2.81(-6) \\
4.46(-5) \\
2.81(-7) \\
1.62(-5) \\
5.62(-6) \\
5.62(-7) \\
2.51(-6) \\
7.07(-7) \\
3.54(-7) \\
2.50(-5) \\
2.23(-7) \\
3.38(-6)\end{array}$ & $\begin{array}{l}1.008 \\
4.003 \\
12.011 \\
14.007 \\
15.999 \\
20.179 \\
22.990 \\
24.305 \\
26.982 \\
28.086 \\
30.974 \\
32.060 \\
39.948 \\
39.102 \\
40.080 \\
51.996 \\
54.938 \\
55.847 \\
58.933 \\
58.710\end{array}$ & $\begin{array}{r}13.60 \\
24.58 \\
11.26 \\
14.53 \\
13.61 \\
21.55 \\
5.14 \\
7.64 \\
5.98 \\
8.15 \\
10.48 \\
10.35 \\
15.75 \\
4.33 \\
6.11 \\
6.76 \\
7.43 \\
7.83 \\
7.86 \\
7.63\end{array}$ \\
\hline
\end{tabular}

Adapted from Vernazza et al. (1973) 
of about $6000 \mathrm{~K}$. Double ionisations have been neglected as a source of electrons since at the temperatures where these become important, hydrogen is completely ionised and thus the major electron donor by virtue of its abundance. Ionisation of helium, the second most abundant element, is significant near the coronal base ( $\mathrm{Te} \sim 10,000 \mathrm{~K}$ ) where hydrogen is fully ionised.

The above 4 independent equations in the 4 unknowns, $\mathrm{Pg}, \rho, \mathrm{Nh}$ and $\mathrm{Ne}$, are simultaneously solved at each atmospheric depth point using the iterative scheme of Gray (1976). The LTE hydrogen populations and proton density (NP) subsequently follow from equation 4.1 and the Saha-Boltzmann relation.

In the case of calcium, its total number density is calculated from $\mathrm{Nh}$, according to a specified calcium abundance $[\mathrm{Ca} / \mathrm{H}]$. The corresponding LTE level populations are then derived from the Saha-Boltzmann distribution, with the appropriate atomic parameters (Table 4.3).

\subsection{CONTINUUM AND LINE OPACITY CALCULATIONS}

\subsubsection{Continuum Opacity Sources}

In solving the non-LTE transfer problem, background opacities and emissivities are required at each depth point in the model atmosphere, and for each frequency in the transition under investigation. We present here a summary of the major continuum sources of opacity included in the profile synthesis code. 


\section{(a) Atomic hydrogen}

Since hydrogen is the dominant atmospheric constituent, its bound-free (B-F) and free-free ( $F-F)$ absorptions play an important role in the control of emergent energy flux of a stellar atmosphere. For example, in the upper solar chromosphere ( $\mathrm{Te} \sim 7000 \mathrm{~K}$ ) photoionisations from the $n=3$ level (Paschen continuum) contribute up to 50 \& of the total continuous opacity in the wavelength region of $\mathrm{H}-\mathrm{al}$ pha.

For the 5-level model hydrogen atom the B-F cross-section at frequency $v$ for the $i$ th bound level can be written,

$$
\begin{aligned}
\alpha_{i}(\mathrm{H}) & =2.815 \mathrm{E} 29 \mathrm{gBF} / \mathrm{\nu}^{3} / \mathrm{i}^{5} & & \nu>3.29 \mathrm{E} 15 / \mathrm{i}^{2} \\
& =0 & & \text { otherwise }
\end{aligned}
$$

where $\mathrm{gBF}$ is the $\mathrm{B}-\mathrm{F}$ gaunt factor (obtained from the polynomial expansions in Mihalas, 1967a).

The non-LTE B-F opacity (per gram) is derived from the following summation over the hydrogen energy levels,

$$
X_{B F}(H)=\frac{1}{\rho} \sum_{i=1}^{5} a_{i}(H) N i \quad\left\{\frac{-\exp (-h \nu / k T)\}}{b i}\right.
$$


and the corresponding emissivity is given by,

$$
E_{B F}(H)=\frac{1}{\rho} \sum_{i=1}^{5} \alpha_{i}(H) \quad N i \frac{2 h \nu^{3}}{c^{2}} \frac{\exp (-h \nu / k T)}{b i}
$$

During the initial set-up phase, the hydrogen departure coefficients, bi, (defined as the ratio of non-LTE to LTE atomic populations) are unity, hence the above expressions assume their LTE forms. With subsequent iterations, the hydrogen populations are updated with non-LTE estimates (as outlined in section 4.8.3) and the opacities and emissivities re-calculated accordingly.

The F-F opacity term is also incorporated since, at very large temperatures and long wavelengths, its cross-section becomes comparable to that of the B-F. As the interaction is collisional in character, its opacity contribution is computed in LTE by employing the relation,

$$
\begin{gathered}
x_{F F}(H)=-\frac{1}{\rho} \frac{N e N p}{\sqrt{T}} \frac{3.69 E 8}{\nu^{3}}\left[g F F-1.0+\exp \left(-h \nu_{F F} / k T\right)\right] \\
v_{F F}=\min [\nu, 9.138 \mathrm{E} 13 \mathrm{~Hz}]
\end{gathered}
$$

where $g F F$ is the F-F gaunt factor which is determined from the formula given by Mihalas (1967b). The associated emissivity is obtained by multiplication of equation 4.9 with the Planck function.

\section{(b) Negatively ionised hydrogen, $\mathrm{H}^{-}$}

Because of its relatively large absorption cross-section, $\mathrm{H}^{-}$is an extremely important source of continuous opacity in late type stellar photospheres, 
absorbing radiation via both $B-F$ and $F-F$ interactions. For example, in the temperature minimum region of the solar chromosphere, the former process accounts for almost $9 \varnothing$ of of the total background opacity in the wavelength region of $\mathrm{H}-\mathrm{al}$ pha. The F-F component only becomes appreciable at very large wavelengths (>15000 $\AA$ ). LTE is assumed in the calculation of the net $\mathrm{H}^{-}$ opacity, as it has been shown to be a good approximation for $\mathrm{H}^{-}$in the lower solar chromosphere (Vernazza et al., 1976). Thus,

$$
X\left(H^{-}\right)=\alpha\left(H^{-}\right) n^{\star}\left(H^{-}\right)[1-\exp (-h \nu / k T)]
$$

where the exponential term is the correction for stimulated emission and $\mathrm{n}^{*}\left(\mathrm{H}^{-}\right)$the LTE number density derived from the Saha-Boltzmann equation.

The absorption cross-section, $\alpha\left(\mathrm{H}^{-}\right)$, is calculated using the polynomial fits of Gingerich et al., (1967) to the $B-F$ and F-F tabulations of Geltman (1962) and John (1964), respectively. Multiplication of equation 4.10 by $\mathrm{Bv}(\mathrm{T})$ yields the $\mathrm{H}^{-}$emissivity.

\section{(c) Bound-free continua of metals}

The photoionisation continua of neutral metals play a predominant role in the opacity over the wavelength range 1500-3000 $\AA$, at temperatures near $5000 \mathrm{~K}$. Of the various elements with comparatively high abundances in solar-type stars, Mg, Si, $\mathrm{Ca}$ and $\mathrm{Al}$ are the major contributors to the metallic B-F absorption distribution. The opacity due to these species is calculated in the 
hydrogenic approximation (Gingerich, 1967), using the photoionisation cross-sections tabulated in Gingerich and Rich (1967).

B-F absorption of Carbon I is of considerable importance in the wavelength region of the Lyman lines, accounting for most of the continuous opacity in the photosphere and middle chromosphere. Its contribution to this background is determined from the exponential fit of Gingerich (1967) to the data of Peach (1967).

\section{(d) Continuum scattering opacity}

Thompson scattering of radiation from free electrons and Rayleigh scattering from neutral hydrogen are both included in the background opacity contribution. The cross-section for the former process is computed from the classical grey formula,

$$
\alpha_{S}\left(e^{-}\right)=\frac{8 \pi e^{4}}{3 m_{e^{2} c^{4}}}=6.65 E-25 \mathrm{~cm}^{2}
$$

This opacity source is important in the hottest atmospheric layers.

Rayleigh scattering is prominent at relatively short wavelengths and can play a significant role in the higher chromospheric layers where $\mathrm{H}^{-}$is unimportant. Moreover, in metal weak stars, the reduction in electron supply from photospheric metals diminishes the opacity from $\mathrm{H}^{-}$, thus enhancing the importance of the scattering term. The cross-section for this interaction is derived from 
the expression of Dalgarno (1964),

$$
\alpha_{S}(H)=\frac{5.799 E-13}{\lambda^{4}}+\frac{1.427 E-6}{\lambda^{6}}+\frac{2.784}{\lambda^{8}} \quad[\lambda: A] \quad(4.12)
$$

The total scattering opacity per gram follows from,

$$
X s=\left[N e \alpha_{s}\left(e^{-}\right)+N h \alpha_{s}(H)\right] / p
$$

and the emissivity from the coherent scattering assumption,

$$
E s=X s \mathrm{JV}
$$

where $J v$ is the mean intensity (defined in section 4.6).

\subsubsection{Line Opacities}

Depth-dependent absorption cross-sections for the line transitions treated in detail are computed at each frequency point in the profile according to the formula,

$$
\alpha_{i j}=\left\{\frac{\sqrt{\pi} e^{2}}{m_{e} c}\right\} \frac{f i j}{\Delta \nu_{D}} H(a, v)
$$

The terms in this expression are defined as follows:

(a) fij are the transition oscillator strengths, which have been tabulated in section 4.3 .

(b)

$$
\Delta v_{D}=\frac{v_{0}}{c}\left\{\frac{2 k T}{m_{H}^{A}}+v^{2}\right\}^{1 / 2} \quad\left[\sec ^{-1}\right]
$$

is the line Doppler width containing turbulent and thermal broadening terms, with $A=1.008$ and 40.08 for $H$ 
and $\mathrm{Ca}$, respectively.

(c) $\mathrm{H}(\mathrm{a}, \mathrm{v})$ is the voigt function which is computed at each frequency point in the line via the series expansions of harris (1968). This function can be represented schematically by,

$$
H(a, v)=\exp \left(-v^{2}\right)+\frac{a}{\sqrt{\pi} v^{2}} \quad(a<<1) \quad(4.17)
$$

where the first term applies in the line core $\left(\nu<3 \Delta \nu_{D}\right)$ and the second in the line wing $\left(\nu>3 \Delta \nu_{D}\right)$. The core is clearly contrclled by Doppler broadening, while the wings are dominated by the damping profile.

(d)

$$
a=\Gamma / 4 \pi \Delta v_{D}
$$

where $I$ is the damping parameter given by,

$$
\Gamma=\Gamma r a d+\Gamma r e s+\Gamma s t a r k+\Gamma v d w
$$

and rrad the radiative damping width deduced from the sum of spontaneous transition probabilities,

$$
\operatorname{rrad}=\sum_{i<U} A_{U i}+\sum_{i<L} A_{L i}
$$

where $L$ and $U$ designate the transition lower and upper levels, respectively.

The resonance broadening parameter, rres, is evaluated for the hydrogen lines using the formula of Breene (1957), 


$$
\text { Tres }=4 / 3 N_{1} \frac{e^{2}}{m_{e} \nu_{0}} f i j
$$

where $\mathrm{N}_{1}$ is the number density of ground state hydrogen. Zelenka (1974) has shown this line broadening process to be important for the solar photospheric H-alpha profile, however it is negligible for the Ca II transitions. Stark broadening due to electrons is derived for H-alpha using the expression of sutton (1978),

$$
\text { Istark, } \mathrm{H}_{\alpha}=0.818(\mathrm{Ne})^{2 / 3}
$$

The approximation of Stark broadening by a Lorentzian, implicit in the use of a voigt function, does not yield the correct Holtsmark distribution in the profile wings. However, this assumption will not lead to serious errors in the line core, where Doppler motions essentially dominate the line broadening. In the solar chromosphere, Istark for the Lyman and Ca II lines is negligible compared to rrad (Vernaza et al., 1980; Shine and Linsky, 1974).

Van der Wals (VDW) broadening due to collisions with neutral hydrogen is determined using the approximate discrete encounter relation (Mihalas, 1978),

$$
\text { IVdW }=\text { KO Nh }(\mathrm{m})^{1 / 3}
$$

Values of Ko for the calcium lines are taken from shine and Linsky (1974), who have demonstrated the importance of VDW broadering in the photospheric wings of $\mathrm{H}$ and $\mathrm{K}$. For the hydrogen lines, in particular H-alpha, rvdw is small compared to rrad or rres and is, therefore, 
neglected.

(e)

$$
v=\frac{v-v_{0}}{\Delta v_{D}}
$$

and denotes the shift from line centre of a frequency grid point, in units of the Doppler width. In the case of the hydrogen lines and the Ca II IR triplet, 15 frequency points are typically used over a half bandwidth such that vmax=iø. This grid provides adequate coverage of the line cores and wings. For the Ca II resonance doublet, the bandwidth is extended to $\operatorname{vmax}=100$, with 20 frequency points logarithmically spaced so as to achieve high resolution in the line cores, whilst encompassing the broad damping wings ( $15 \AA$ wide).

The cross-section for each line transition is normalised such that,

$$
2 \sum_{\nu=1}^{N F} \alpha_{\nu} W_{\nu}=1.0
$$

where Wv are trapezoidal quadrature weights (the grid spacing between adjacent frequency points) and NF the number of frequency points per transition spanning the half bandwidth. The factor of 2 is derived from the symmetry of the line profile about line centre. For the Lyman continuum transition, the absorption cross-section is calculated at 9 wavelength points, equispaced between $612 \AA$ and $912 \AA$, using equation 4.6. with $i=1$.

Finally, the background opacities computed in 
section 4.5 .1 are combined with the line opacity terms to produce, for a transition at frequency $v$, the total absorptivity (neglecting overlapping transitions).

$$
\lambda_{\nu}=x_{v}+X_{s}+\alpha_{i j}(N i-G i j \cdot N j) / 0
$$

the total emissivity,

$$
\eta_{\nu}=E_{\nu}+X s J \nu+\frac{2 h \nu^{3}}{c^{2}} \alpha_{i j} \frac{G i j \cdot N j}{\rho}
$$

and the source function,

$$
S v=\frac{n_{v}}{\lambda_{v}}
$$

(depth indices have been suppressed for convenience)

In the above, $\mathrm{Ni}$ and $\mathrm{Nj}$ denote number densities

(initially in LTE) of atoms ( $H$ or $C a$ ) in the lower and upper levels respectively for the transition, while Gij is given by,

$$
\begin{aligned}
\mathrm{Gij} & =\mathrm{g}_{\mathrm{i}} / \mathrm{g}_{j} & & \text { for a line transition } \\
& =\mathrm{Ni}^{*} / \mathrm{Nj}^{*} \exp (-\mathrm{hv} / \mathrm{kT}) & & \text { for a photoionisation }
\end{aligned}
$$

$\left(\mathrm{Nj}^{*}\right.$ corresponds to the proton density in the case of the Lyman continuum)

\subsection{FEAUTRIER SOLUTION OF RADIATIVE TRANSFER EQUATION}

$$
\text { Utilising the notation of Feautrier (1964), the }
$$

plane-parallel transfer equation can be cast into the second order differential form,

$$
\mu^{2} \frac{\delta^{2} u_{\mu \nu}}{\delta \tau \nu^{2}}=u_{\mu \nu}-s \nu \quad \mu=\cos \theta
$$


where $u_{\mu \nu}$ is the mean of outgoing and incoming intensities along rays inclined at an angle $\theta$ to the normal z direction,

$$
u_{\mu \nu}=\frac{\{I(z, \mu, \nu)+I(z,-\nu, \nu)\}}{2}
$$

and $\tau_{v}$ corresponds to the optical depth scale, which is related to the geometrical height $(z)$ and mass column density via,

$$
\delta \tau_{\nu}=-\lambda_{\nu} \rho \delta z=-\lambda_{\nu} \delta m
$$

Equation 4.29 is converted into a set of ND (the total number of depth points) finite-difference relations by replacement of differentials with difference formulae such that,

$$
\begin{aligned}
& \left\{\frac{\mu^{2}}{\Delta \tau_{d-1 / 2} \Delta \tau_{d}}\right\} u_{\nu \nu, d-1}-\frac{\mu^{2}}{\Delta \tau}\left\{\frac{1}{\Delta \tau d-1 / 2}+\frac{1}{\Delta \tau_{d+1 / 2}}\right\} u_{\mu \nu, d} \\
& \quad+\left\{\frac{\mu^{2}}{\Delta \tau_{d} \Delta \tau_{d+1 / 2}}\right\} u_{\mu \nu, d+1}=u_{\mu \nu, d}-s_{\nu, d} \\
& \Delta \tau_{d+1 / 2} \simeq \frac{\left(\lambda_{\nu, d+1}+\lambda_{\nu, d}\right)}{2} \cdot\left(m_{d+1}-m_{d}\right)
\end{aligned}
$$

The mean intensity in the coherent scattering integral is defined by,

$$
J \nu=\frac{1}{2} \int_{-1}^{1} I_{\nu} d \mu=\int_{j}^{1} u_{\mu \nu} d \mu
$$

and is evaluated by a quadrature sum with respect to three angular points using gaussian weights.

Upon collection of terms in $u_{\mu \nu}$ and writing the 3 angular components as a column vector $U_{d}$, the set of 
discretised transfer equations assume the matrix forms,

$$
\begin{gathered}
B_{1} \cdot U_{1}-C_{1} \cdot U_{2}=L_{1} \\
-A_{d} \cdot U_{d-1}+B_{d} \cdot U_{d}-C_{d} \cdot U_{d+1}=L_{d} \\
-A_{N D} \cdot U_{N D-1}+B_{N D} \cdot U_{N D}=L_{N D}
\end{gathered}
$$

where $A_{d}, B_{d}$ and $C_{d}$ are 3 by 3 matrices containing the finite difference representations of the differential operator and the quadrature sums, and $L_{d}$ is a column vector consisting of the thermal source terms. Detailed expressions for the components of these matrices are provided by Mihalas (1978).

Equations $4.35 \mathrm{a}$ and $\mathrm{c}$ are derived from second order finite differencing of the upper $(d=1)$ and lower ( $d=N D$ ) boundary constraints respectively. The former relation arises from the condition of zero incident intensity (I(0) at the top of the model atmosphere (Aver, 1967). The latter follows from the diffusion approximation according to which, at photospheric depths much larger than a photon mean free path, the radiation is effectively trapped and asymptotically approaches thermal equilibrium (Auer and Mihalas, 1969). Under this circumstance, the upward intensity is given by,

$$
I^{+}=B \nu(T)+\left.\mu \frac{\delta B \nu(T)}{\delta \tau \nu}\right|_{N D}
$$

The simultaneous solution of the matrix formulae proceeds, for each transition frequency, as follows. 
Equations $4.35 \mathrm{a}, \mathrm{b}$ and $\mathrm{c}$ are organised into the form,

$$
\mathrm{U}_{\mathrm{d}}=\mathrm{D}_{\mathrm{d}} \cdot \mathrm{U}_{\mathrm{d}+1}+\mathrm{V}_{\mathrm{d}}
$$

where,

$$
D_{d}=\left(B_{d}-A_{d} \cdot D_{d-1}\right)^{-1} \cdot C_{d}
$$

and,

$$
v_{d}=\left(B_{d}-A_{d} \cdot D_{d-1}\right)^{-1} \cdot\left(L_{d}+A_{d} \cdot v_{d-1}\right)
$$

Commencing at $d=1$, successive values of $D d$ and vd are computed through to $d=N D-1$. At the final depth point $C_{d}=\emptyset$, and hence $D_{d}=\emptyset$ and $U_{d}=v_{d}$. Successive back-substitutions are then performed on equation 4.37 a to obtain $U_{d}(d=N D-1, \ldots .2,1)$. These are subsequently used to evaluate JV (eq. 4.34) and the emergent flux (at d=1) from the integral,

$$
F \nu=\frac{1}{2} \int_{-1}^{1} I_{\nu} \mu d \mu
$$

Finally, the variable Eddington factors ( $f v)$, which enter into the Linearisation procedure described in section 4.8 .2 , are deduced from the ratio,

$$
f v=K v / J v
$$

where

$$
K \nu=\frac{1}{2} \int_{-1}^{1} I_{\nu} \mu^{2} d \mu
$$


4.7 FORMULATION OF THE RATE EQUATIONS

\subsubsection{Radiative Rates}

\section{(a) Explicit transitions}

The radiative rates per atom ( $R i j)$, for the transitions treated in detail, are determined during the course of the radiative transfer solution as follows.

The upward rate is given by,

$$
R i j=4 \pi \int \frac{\alpha_{i j}}{h v} J v d v
$$

and the downward rate (incorporating spontaneous and stimulated emissions) by,

$$
R j i=4 \pi \int \frac{G i j}{h v} \alpha_{i j}\left(\frac{2 h v^{3}}{c^{2}}+J v\right) d v
$$

where Gij is defined in section 4.5 and $\alpha_{i j}$ corresponds to the line (or continuum) transition cross-section. The above frequency integrations are carried out via trapezoidal quadrature across the profile bandwidth.

\section{(b) Fixed transitions}

In addition to the above transitions, various interactions between the upper $\mathrm{H}$ (and $\mathrm{Ca}$ ) levels are approximately specified at the outset of the transfer calculation in terms of fixed rates. Provided the relevant transitions are optically thin, these rates are intrinsic to the stellar spectral energy distribution and are not affected by changes in level populations (Ayres and Linsky, 1975a).

Following the convention of Auer et al. (1972), a 
fixed radiation field is characterised by a radiation temperature, $\operatorname{Tr}$, such that $\mathrm{J} \nu=\mathrm{B} \nu(\mathrm{Tr})$ in the

chromosphere, where the transition is deemed optically thin, and $J \nu=B \nu(T e)$ in the photosphere, where the transition source function is thermalised. Values of $\operatorname{Tr}$ are dependent upon background photospheric opacities and emissivities, in particular line-blanketing distribution functions, and can be empirically estimated from calibrated spectral energy distributions (see Ayres and Linsky, 1975 a for examples related to the sun and Arcturus).

Substitution of $\mathrm{J} v(\operatorname{Tr})$ into equation 4.41 yields, for a photo-excitation,

$$
R i j=\frac{4 \pi}{h v} \frac{\pi e^{2}}{m c} f i j B v(T r)
$$

and for a photoionisation,

$$
\text { Rik }=\frac{8 \pi}{c^{2}} \alpha_{\nu 0} \nu_{0}^{3} \text { EI }\left\{\frac{h \nu_{0}}{k \operatorname{Tr}}\right\}
$$

where $E l(x)$ is the first exponential integral evaluated from the series expansions of Abramowitz and Stegun (1968), and $\alpha_{v 0}$ is the hydrogenic B-F cross-section (cf. equation 4.6) evaluated at the head of the continuum. Analogous formulae for the radiative de-excitation and recombination rates are provided by Auer et al. (1972) and Ayres and Linsky (1975 a).

The hydrogen transitions treated according to this formalism comprise the Balmer, Paschen, Brackett and Pfund continua, and the $3-4,3-5$, and $4-5 \quad B-B$ 


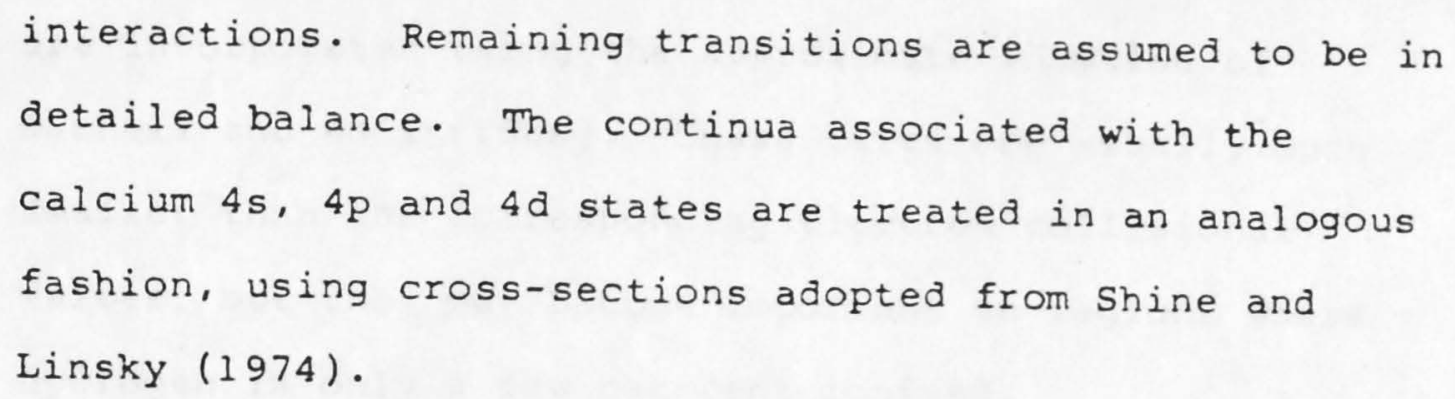

\subsubsection{Collisional Rates}

The electron collisional excitation and ionisation rates ( $C i j$ ) for the $\mathrm{H}$ and $\mathrm{Ca}$ atomic levels are calculated at each depth point in the model atmosphere according to the formula,

$$
C_{i j}=\operatorname{Ne} \Gamma_{i j}(T)
$$

where rij denotes the temperature dependent electron collisional cross-section for an upward transition.

Cross-sections for hyorogen $B-B$ interactions are deduced from the semi-empirical formulae of Sampson and Golden (1970) and Golden and Sampson (1971). Hydrogen ionisation cross-sections are evaluated using the equations of Mihalas (1967a), which are based on the data of Kieffer and Dunn (1966) and Percival (1966).

Electron collisional cross-sections for the $\mathrm{Ca}$ II $\mathrm{H}$ and $K$ lines are taken from the experimental values of Taylor and Dunn (1973), while the theoretical results of Saraph (1970) are employed for the remaining B-B cross-sections. The B-F collisional rates are determined from the approximate equation of Shine and Linsky (1974). Couplings between the fine structure terms $(4 \mathrm{P} 1 / 2-4 \mathrm{P} 3 / 2$, $3 D 3 / 2-3 D 5 / 2$ ) induced by collisions with neutral hydrogen, 
are incorporated using the approximate formulae of Bachall and wolf (1968). These rates are usually much smaller than the corresponding electron collisional values, but they may become important in regions where hydrogen is only a few per cent ionised.

Finally, collisional de-excitation and recombination rates are derived from the principle of detailed balance,

$$
C j i=\operatorname{Cij} \frac{N i^{\star}}{N j^{\star}}
$$

\subsubsection{The Equations of Statistical Equilibrium}

The statistical equilibrium equations (or rate equations) describe the balance between processes (radiative and collisional) that populate an atomic energy level with those that de-populate it. In a steady state situation, the rates at which these processes occur are equal such that the level populations are constant with time. For a level $i$, in an atom with $\mathrm{N}$ bound levels and a continuum $(k)$, the statistical equilibrium equation can be formulated as,

$$
\text { Ni } \underset{m \neq i}{N} \operatorname{Pim}+P i k)=\sum_{m \neq i}^{N} N m P m i+N k P k i
$$

where $\mathrm{Pij}=\mathrm{Rij}+\mathrm{Cij}$; $\mathrm{Ni}$ and $\mathrm{Nm}$ are bound level number densities and $N k$ is the number density of ionised atoms (protons in the case of $\mathrm{H}, \mathrm{Ca}$ II in the case of $\mathrm{Ca}$ ). A similiar expression can be written for the ionic species however it is redundant with the bound level equations, (as can be shown by summation of equation 4.47 over the $N$ 
states).

The above system of equations is closed by the requirement of number conservation,

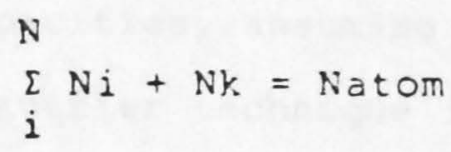

where Natom is the total number density of atoms. Writing the bound and continuum populations as a column vector, N, equations 4.47 and 4.48 can be cast into the matrix form,

$$
A \cdot N=B
$$

where $A$ is an $N+1$ by $N+1$ matrix containing the components,

$$
\begin{aligned}
A_{i j} & =-R_{N} \text { Ri-Cji } \\
& =\sum_{k \neq i}(R i k+C i k) \\
& =0 \\
& =1
\end{aligned}
$$

$$
\begin{aligned}
& j \neq i \neq N+1 \\
& j=i \neq N+1 \\
& i=N+1, j \neq N+1 \\
& i=N+1, j=N+1
\end{aligned}
$$

and $B$ a vector with,

$$
\begin{aligned}
B_{i} & =0 & & i<N+1 \\
& =N a t o m & & i=N+1
\end{aligned}
$$

Given the transition rate parameters and the total atomic density, equation 4.49 can be inverted to yield bound level and continuum populations that are consistent with the rate equations. 


\subsubsection{Lambda Iteration}

To initiate the computation of non-LTE level populations, the model atmosphere is used to determine line and continuum opacities, assuming LTE for the atom under study. The Feautrier technique is then employed to calculate the radiation fields for each transition under investigation. These fields are subsequently used to derive radiative rates which are combined with collisional and fixed rates into the statistical equilibrium equations. Solution of the latter leads to a new set of atomic populations which are used to re-evaluate the transition opacities and emissivities. The Feautrier calculation is then repeated to produce updated radiation fields and rates.

This recursive procedure is referred to as a lambda iteration and can, in principle, be used to obtain a consistent simultaneous solution of the radiative transfer and statistical equilibrium equations. However, as is often characteristic of non-LTE transfer, the source function contains a dominant scattering term that is only weakly coupled to local thermal conditions. Under these circumstances, Su may differ greatly from Bv over large ranges of optical depth. It can be shown (Mihalas, 1978) that each successive lambda iteration can transmit information concerning the departure of $\mathrm{Sv}$ from Bu only over a photon mean free path. Hence, depending upon the magnitude of scattering thermalisation lengths, the convergence rate of the lambda iteration (with initial LTE population estimates) can be extremely slow, often requiring a prohibitively large number of 
iterations to propagate information on the existence of an external boundary to the photospheric layers.

The poor convergence of this technique is

exacerbated when the effects of interlocking among the

transitions of a multilevel atom are considered. As is

evident from the statistical equlibrium equations, a

change in the radiation field at any frequency implies a

change in the occupation numbers of all the atomic

levels. Furthermore, alterations in the level

distribution induce changes in the emissivity and opacity of the atmospheric medium and consequently, via the transfer equation, the radiation field throughout the atmosphere.

A more efficient and powerful scheme for the solution of non-LTE line transfer is provided by the Complete Linearisation Method of Auer and Mihalas (1969). In essence, the method involves the development of a system of linear equations by perturbation of the radiation fields and atomic number densities intrinsic to the coupled transfer and rate equations. An iterative, simultaneous solution of these equations fully accounts for the global nature of the transfer equation, and its complex interplay with the statistical equilibrium equations.

\subsubsection{The Complete Linearisation Method}

We present here a brief description of the Linearisation technique as applied to the simplified case of a model atmosphere of given temperature structure and 
fixed gas, electron and total atomic number densities. (The treatment of variations in the latter densities is described in section 4.8.3). More complete discussions of the methodology are given by Auer et al. (1972), Auer (1973). Auer and Heasley (1976) and Mihalas (1978). Following the approach of Auer and Mihalas (1970), the angular dependence of equation 4.29 is eliminated by integration over $\mu$ and introduction of the variable Eddington factor, fv. Hence,

$$
\frac{\delta^{2}(f \nu J \nu)}{\delta \tau \nu^{2}}=J \nu-S \nu
$$

Finite differencing of this expression at each depth point, including the upper and lower boundaries, leads to a set of ND relations analogous to equations $4.37 \mathrm{a}, \mathrm{b}$ and $c$. The discretised system is linearised with respect to $J_{\nu}$ and the level populations by first order perturbations of the form :

$$
\mathrm{J \nu}=\mathrm{J} \nu_{0}+\delta \mathrm{J \nu} \text { and } \mathrm{Ni}=\mathrm{Ni} i_{0}+\delta \mathrm{Ni}
$$

where the perturbed quantities are functionally related by,

$$
\delta N i=\sum_{\nu} \frac{\delta N i}{\delta J \nu} \delta J \nu
$$

and the summation is taken over the total number of frequency grid points (NFT) covered by all the transitions being considered. Analytic formulae for $\frac{\delta \mathrm{Ni}}{\delta \mathrm{J} v}$ are derived by a similiar perturbation of the statistical 
equilibrium equations (Aver, 1973).

Substitution of equation 4.53 into the linearised

transfer equations yields the Feautrier-like system,

$$
\begin{gathered}
-A_{d} \cdot \delta J d-1+B_{d} \cdot \delta J_{d}-C_{d} \cdot \delta J d+1=L_{d} \\
A_{1}=C_{N D}=0
\end{gathered}
$$

where $\delta J_{d}$ is a column vector comprising the NFT

corrections to the radiation field at depth d; $A_{d}, B_{d}$ and $C_{d}$ are square matrices of dimension NFT containing quantities associated with perturbed opacities and emissivities, and $\mathrm{L}_{\mathrm{d}}$ is a vector consisting of the zeroth order terms from the perturbation. Exact expressions for these matrix components are given in the references cited above.

The Feautrier forward-elimination and backward-substitution scheme (as outlined in section 4.6) is employed for the solution of equation 4.54. New radiation fields and corresponding radiative rates are then derived, at each depth point, by application of the corrections to the original fields computed from the initial lambda iteration. The updated rates are subsequently utilised in the generation of a new set of level populations from the rate equations. Opacities and emissivities are re-calculated and a second lambda iteration performed to obtain radiation fields and Eddington factors, (this step also provides a smoothing of the solution). The linearised transfer equations are then re-solved for $\delta J_{\mathrm{d}}$ and the procedure repeated. The convergence of the linearisation-lambda sequence is both 
swift and stable, typically yielding

$$
\left|\frac{\delta J v}{J \nu}\right|<10^{-5}
$$

in 5 or 6 iterations.

4.8.3 Hydrostatic Equilibrium Adjustment of Hydrogen And Electron Number Densities

Non-LTE radiative transfer in the hydrogen

transitions is complicated by the fact that hydrogen is

the dominant atmospheric constituent and thus the primary source of chromospheric electrons. Consequently, changes in the non-LTE hydrogen ionisation balance will, via the equation of state, influence the chromospheric density structure and, in turn, the transfer calculation of radiation fields. A complete solution of the transfer equation therefore requires that the non-LTE hydrogen bound level and proton populations be consistent with electron densities. This compatability is effected in the following manner.

The particle conservation (eq. 4.48) and charge neutrality (eq. 4.5) expressions are re-formulated as,

$$
\begin{gathered}
N P=N h-\sum_{i}^{N} N i \\
N e=N P+N h \sum_{2}^{N} \frac{A_{i}}{1+N e \psi_{i}(T)}
\end{gathered}
$$

where the assumption of LTE ionisation for the non-hyarogen species in Table 4.5 is retained. Together with the HSE constraint (eq. 4.2), and equations 4.3 and 4.4 , these relations comprise an independent 
system in the 5 unknowns, Ne, Np, Nh, $\rho$ and $\mathrm{Pg}$. Using starting solutions of $\mathrm{Ne}, \mathrm{Nh}, \rho$ and $\mathrm{Pg}$ (derived from the initial LTE calculation) and $\mathrm{Ni}$ and $\mathrm{Np}$ (deduced from the rate equations), the set of equations is solved via a Newton-Rhapson recursive scheme.

During the course of the iterations, successive values of $\mathrm{Nh}$ are used to compute new LTE level populations from the Boltzmann equation (eq. 4.1)., and departure coefficients according to the customary Menzel (1937) definition,

$$
\mathrm{bi}=\frac{\mathrm{Ni} / \mathrm{Ni}^{\star}}{\mathrm{Nk} / \mathrm{Nk}^{*}} \quad: \quad \mathrm{bk}=1
$$

These are combined with current estimates of He and Np to derive self-consistent non-LTE atomic populations using the non-LTE analog of the Saha-Boltzmann distribution,

$$
\mathrm{Ni}=\mathrm{Ne} \mathrm{Np} \text { bi }{ }_{\mathrm{Hi}}(\mathrm{T})
$$

The updated level populations are subsequently incorporated in the summation of equation 4.55 and the iterations repeated until convergence. Usually, 5 to 6 recursions are required to yield number densities accurate to within 18 .

\subsection{OVERVIEW OF RADIATIVE TRANSFER CALCULATION}

This concluding section presents an outline of the convergence procedure for the hyorogen and calcium transfer solutions.

A converged solution of the coupled transfer, 
statistical and hydrostatic equlibrium equations for the hydrogen atom was obtained within 2 to 3 hours of Univac CPU time, the bulk of which was spent in the inversion of frequency matrices during the linearisation solution. Following every fourth linearisation-lambda iteration, the atmospheric density structure was adjusted via a HSE calculation. Background opacities and emissivities were re-evaluated from the new model atmosphere, and collisional rates re-computed using updated electron densities. New radiation fields, radiative rates (including fixed rates) and non-LTE level populations were then obtained from a lambda iteration. A second hyàrostatic adjustment and smoothing lambda iteration were performed, and the linearisation-lambda sequence repeated.

The series of calculations was terminated when, $\operatorname{Max}\left|\frac{\delta J v}{J v}\right| \leqslant 10^{-7}$

was reached at all depth points in the model atmosphere, and for all transition frequencies. Typically, 12 linearisation-lambda recursions, and 6 nested hydrostatic adjustments were required for simultaneous convergence in the Lyman continuum, Ly-alpha, Ly-beta and H-alpha transitions.

The converged hydrogen populations and electron densities were then utilised in the computation of background opacities, emissivities and collisional rates, necessary for the transfer solution in the $\mathrm{Ca}$ II $\mathrm{H}$ and $\mathrm{K}$, and IR triplet lines. Commencing with LTE atomic calcium 
populations, simultaneous convergence in these transitions was achieved in 4 to 5 hours of CPU, with 7 to 8 linearisation-lambda iterations.

In many instances, the linearisation procedure developed convergence instabilities that were manifested as spurious "bumps" or glitches in the height distribution of number densities. This difficulty was partly due to the effects of machine round-off errors generated in the pivoting process of the matrix inversion routine. Generally, the growth of these errors was attenuated by subsequent lambda iterations. However, in certain circumstances, the density discontinuities were amplified following successive linearisations, and precipitated serious divergence of the iterative procedure. This complication was found to be caused by rapid depth variations of line core opacities on scales smaller than the specified atmospheric grid spacing. The insertion of additional mass points in the region of the instability usually succeeded in rectifying the problem. 
THE DEPENDENCE OF H-ALPHA ON CHROMOSPHERIC ACTIVITY IN LATE TYPE MAIN SEQUENCE STARS

\subsection{INTRODUCTION}

The correspondence between chromospheric activity, as diagnosed by the Ca II $K$ emission strength, and atmospheric temperatures and densities was studied for the case of solar plages by Shine and Linsky (1974). Their model computations demonstrated that an enhanced transition zone (TZ) pressure, together with a steep temperature increase above the temperature minimum (relative to quiet solar regions), were required to explain the bright Ca II $K$ emission of magnetic active regions. The large temperature gradient, presumably the result of amplified non-radiative heating, increases the degree of hyarogen ionisation in the chromosphere. The consequent increase in electron density, Ne, produces a stronger collisional coupling between the $\mathrm{Ca}$ II $\mathrm{K}$ source function and the chromospheric temperature rise, and thus a brighter core emission.

Extensions of the solar plage work to main sequence stars were undertaken by Kelch et al. (1979), who derived chromosphere temperature models using high resolution, flux calibrated Ca II K line profiles of F, G and $K$ dwarfs. The main feature of these models is that the 
"active chromosphere" stars, defined as those with the largest radiative loss rates in the $\mathrm{Ca}$ II and $\mathrm{Mg}$ II resonance lines, exhibit steeper lower chromospheric temperature gradients than quiet chromosphere stars like Alpha Cen A and B (Ayres et al., 1976).

In the light of the above work, we examine the consequences of variations in chromospheric temperature structure on the behaviour of the H-alpha profile in a late type main sequence star. In particular, our aim is to investigate the relationship between such variations and the apparent $\mathrm{H}-\mathrm{al}$ pha core brightening with increasing $\mathrm{Ca}$ II $\mathrm{K}$ emission strength observed among the active $\mathrm{G}$ and $\mathrm{K}$ dwarf stars analysed in Chapter Three (Figure 3.3). An example of the latter effect is shown in Figure 5.1, where H-alpha and Ca II K profiles are compared for the quiet and active G8 dwarfs, Delta Pav and Xi Boo (A), respectively. The Ca II K profiles are compared on the normalised $\mathrm{Ca}$ II $\mathrm{K}$ wing intensity scale defined in Chapter Two.

It is clear that with the apparent enhancement of $\mathrm{H}$-alpha central residual flux with increasing $\mathrm{Ca}$ II $\mathrm{K}$ emission strength, the H-alpha profile becomes a viable diagnostic of chromospheric activity in its own right. This phenomenon indicates a dependence of $\mathrm{H}-\mathrm{al}$ pha line formation on chromospheric heating, as is presumed to occur in solar magnetic regions.

Under the thermodynamic conditions prevalent in the quiet solar chromosphere, photoionisation and radiative recombination are the dominant processes controlling the 


\section{FIGURE 5.1}

A comparison of $\mathrm{Ca}$ II $\mathrm{K}$ (left panel) and $\mathrm{H} \alpha$ (right panel) profiles for the G8 dwarfs $\xi$ Boo (A) (thin line) and $\delta \mathrm{Pav}$ (thick line). The normalised intensity scale for $\mathrm{Ca}$ II $\mathrm{K}$ is in units of the wing flux at $\Delta \lambda=+6 \AA$, as defined in the text. Ha lines are compared on a continuum flux scale. Note the enhanced $\mathrm{H} \alpha$ residual core brightness of $\xi$ Boo (A) relative to $\delta \mathrm{Pav}$, the former star showing strong $\mathrm{Ca}$ II $\mathrm{K}$ emission relative to the latter. 
formation of H-alpha (Jefferies and Thomas, 1959; Gebbie and Steinitz, 1974). The H-alpha line is classified as radiatively controlled and, unlike the collisionally dominated Ca II $K$ source function, its source function couples not with the chromospheric temperature rise, but with a fixed background temperature related to the colours of the photospheric Balmer and Paschen continua (Mihalas, 1978; Vernazza et al., 1980). One consequence is that the H-alpha core, although formed in the warm layers of the middle chromosphere, appears in absorption. However, Gebbie and Steinitz (1974) have suggested that with sufficiently large increases in Ne, as would occur in high temperature plage and flare regions (Cram and Woods, 1982), electron collisional processes can produce a coupling of the H-alpha profile with the chromospheric thermal structure in the line formation region. In view of this occurrence as a possible explanation for the H-alpha contrasts described above, we investigate the theoretical responses of $\mathrm{H}-\mathrm{al}$ pha and $\mathrm{Ca}$ II $K$ profiles to different chromospheric temperature and electron density configurations.

\subsection{MODEL CALCULATIONS}

We consider four schematic temperature models that are representative of "quiet" and "active" thermodynamic states of a chromosphere adjoined to a G2 dwarf photosphere. The modelling approach follows that described in Chapter Four. Starting with prescribed temperature - mass distributions, the Complete 
Linearisation Method (Auer and Mihalas, 1969) is used to solve iteratively the coupled hydrostatic, statistical equilibrium and non-LTE radiative transfer equations for a five bound level plus continuum model hydrogen atom.

The Lyman continuum, Ly-alpha, Ly-beta and H-alpha lines are explicitly treated in the solution, while the fixed rate transitions described in Chapter Four (section 4.7.1) are calculated using the solar radiation temperatures given by Ayres and Linsky (1975a).

Following the hydrogen calculation, the converged electron and hydrogen populations are incorporated in a transfer solution for the $\mathrm{Ca}$ II $\mathrm{H}, \mathrm{K}$ and IR triplet lines using a solar calcium abundance $[\mathrm{Ca} / \mathrm{H}]$ of $2.4 \mathrm{E}-6$ (Ayres, 1977). Fixed continua in the latter calculation are specified with radiation temperatures assumed equal to the the solar effective temperature. Errors of the order of $200 \mathrm{~K}$ in these background temperatures do not significantly affect the computed Ca II K core flux as this optically thick feature is primarily sensitive to variations in collisional processes rather than radiative (Linsky and Avrett, 1970).

Complete Redistribution (CRD) is assumed throughout the calculations, although partial redistribution (PRD) studies of solar Ly-alpha (Milkey and Mihalas, 1973) and solar Ca II H and $K$ (Shine et al., 1975) have shown that departures from this approximation may lead to large changes in computed wing fluxes. However, since we are primarily interested in the behaviour of the H-alpha central residual flux, the CRD approximation does not 
necessarily compromise the validity of the line core computations, as thermal and non-thermal Doppler motions would essentially completely redistribute photons scattered within three Doppler widths of line centre (Thomas, 1957).

Figure 5.2 shows the run of electron temperature with log mass column density $(M)$ in the models, while their defining characteristics (Tmin, Mmin, To and Mo) are listed in Table 5.1. Model 1 is adapted from the Ayres and Linsky (1975a) model $B$ and is representative of quiescent conditions in the solar chromosphere. The lower photospheric portion $(\mathrm{Teff}=5770 \mathrm{~K}, \log (\mathrm{g})=4.4)$ is similar to the Harvard Smithsonian Reference Atmosphere (Gingerich et al., 1971). The chromospheric segment is characterised by a temperature rise, linear in $\log (M)$, between a temperature minimum of $4450 \mathrm{~K}$ at $\log (\operatorname{Mmin})=$ -1.5 , and an upper boundary temperature of $8 \emptyset \emptyset 0 \mathrm{~K}$ at $\log (M O)=-5.5$. Above this point, the transition to coronal temperatures is simulated by a steep temperature increase to $20,000 \mathrm{~K}$. Included in the model is an isotropic microturbulence which varies linearly in $\log (\mathrm{M})$ from a constant photospheric value of $2 \mathrm{~km} / \mathrm{s}$ to a transition region value of $10 \mathrm{~km} / \mathrm{s}$.

Model 2 is derived from the previous distribution by shifting Mo from $\log \left(M_{0}\right)=-5.5$ to -4.0 , while preserving the $8000 \mathrm{~K}$ boundary temperature. The position and value of Tmin is unchanged. This downward migration of the transition zone to higher pressure and the concomitant increase in chromospheric temperature gradient, 
FIGURE 5.2

Annotated Te-Log(M) distributions for the models in Table 5.1. 


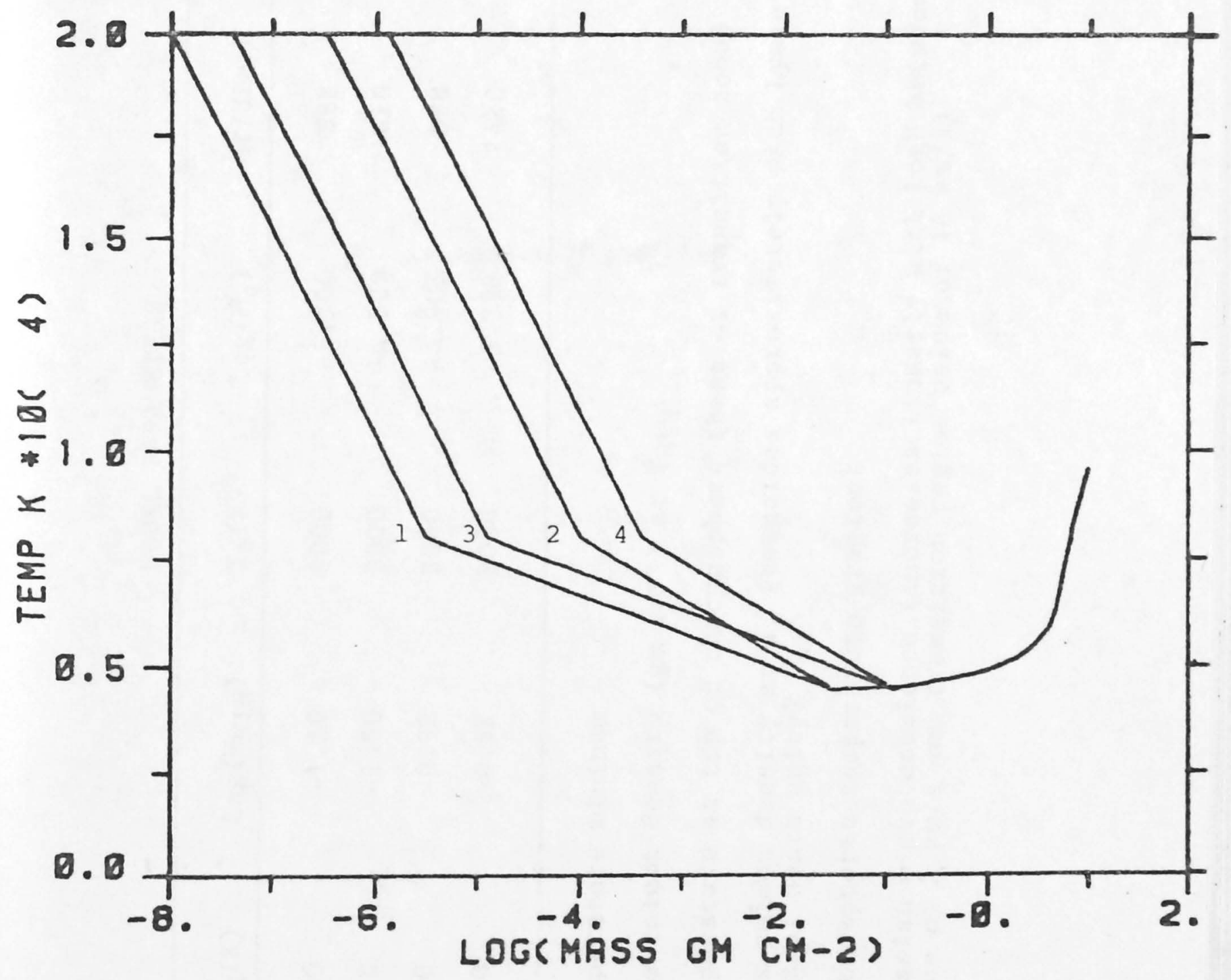




\section{TABLE 5.1}

MODEL PARAMETERS

\begin{tabular}{|c|c|c|c|c|c|c|}
\hline Model & $\mathrm{T}_{\mathrm{MIN}}(\mathrm{K})$ & $\log \left(M_{M I N}\right)$ & $\mathrm{T}_{0}(\mathrm{~K})$ & $\log \left(M_{0}\right)$ & $\mathrm{dT} / \mathrm{dLog} \mathrm{M}$ & $\mathrm{V}_{\mathrm{TURB}}(\mathrm{Km} / \mathrm{s})$ \\
\hline 1 & 4450 & -1.50 & 8000 & $-5 \cdot 500$ & 888 & $2-10$ \\
\hline 2 & 4450 & $-1 \cdot 50$ & 8000 & -4.000 & 1420 & $2-10$ \\
\hline 3 & 4450 & -0.92 & 8000 & $-4 \cdot 908$ & 888 & $2-10$ \\
\hline 4 & 4450 & -0.92 & 8000 & $-3 \cdot 398$ & 1420 & $2-10$ \\
\hline
\end{tabular}

TMIN - Temperature mininum

M MIN - Mass column density $\left(\mathrm{gm}_{\mathrm{cm}}-1\right.$ ) at $\mathrm{T}_{\mathrm{MIN}}$

$\mathrm{T}_{0} \quad$ - Temperature at top of chromosphere (base of transition zone)

Mo - Mass column density at $\mathrm{T}_{0}$ (coincides approximately with Lyman continuum optical depth unity)

$\mathrm{dT} / \mathrm{dLog}$ - Chromospheric temperature gradient

$V_{\text {TURB }}$ - Gaussian microturbulence (increases linearly with LogM between photospheric value of $2 \mathrm{~km} / \mathrm{s}$ and transition region value of $10 \mathrm{~km} / \mathrm{s}$ ) 
$\mathrm{dT} / \mathrm{d} \log (\mathrm{M})$, produces a temperature structure analogous to that considered in the solar plage studies of Shine and Linsky (1974). This temperature model provides a useful paradigm for study of H-alpha formation in an active chromosphere that is heated, for example, by dissipation of acoustic waves possibly interacting with magnetic fields (Ulmschneider, 1979). Alternatively, such a temperature configuration could be the result of upper chromospheric heating by thermal conduction from hot coronal "flare" plasma (Shmeleva and Syrovotskii, 1973). As a consequence of the downward energy flux, the chromosphere-corona interface is forced to lower altitudes than in quiet solar regions (Machado and Linsky, 1975).

Finally, models 3 and 4 are constructed from the quiescent and "plage-like" models by moving Tmin from $\log (\operatorname{Mmin})=-1.5$ to $-\varnothing .92$, whilst maintaining their values of $\mathrm{dT} / \mathrm{d} \log (M)$. These models offer useful test cases with which to examine the $\mathrm{H}-\mathrm{al}$ pha signatures arising from structural changes produced, for instance, by short period acoustic wave heating of the upper photospheric layers. According to the plasma cooling arguments of Ayres (1979), enhanced non-radiative energy input to these layers forces the initial chromospheric temperature inversion to occur at higher densities, than under quiescent conditions. 


\subsection{ANALYSIS OF RESULTS}

Model 1 provides the reference against which we contrast the different morphologies of H-alpha and $\mathrm{Ca}$ II $K$ profiles resulting from the structural changes described in the previous section. Computed H-alpha profiles for models 2,3 and 4 are compared with that of model 1 in Figure 5.3a. A similiar comparison of Ca II $K$ lines is made in Figure $5.3 \mathrm{~b}$. The results of the calculations are summarised in Table 5.2 .

The effect of steepening the chromospheric temperature gradient is to subject more plasma to higher temperatures. The effect of shifting Tmin to larger mass column densities is to place more chromospheric material which is susceptible to ionisation between Tmin and To. Both these perturbations act to increase chromospheric Ne primarily through ionisation of hydrogen in the upper chromosphere, with secondary contributions arising from first ionisations of metals in the lower chromosphere ( Te $\sim 5000-6000 \mathrm{~K})$.

The distribution of $\log (\mathrm{Ne})$ with $\log (M)$ is illustrated in Figure 5.4 for each of the models. As would be expected on the basis of the transfer calculations by Shine and Linsky (1974), the increased chromospheric Ne in models 2, 3 and 4 (see Table 5.2) leads to strong collisional excitation in Ca II $K$ and, consequently, more pronounced emission. Models 3 and 4 also predict broader Kl features compared to their 
FIGURE $5.3 \mathrm{a}$

A comparison of computed $\mathrm{H} \alpha$ profiles from the four chromosphere models (Table 5.1) is made on common velocity and continuum flux scales. Note the strong $\mathrm{H} \alpha$ emission from Model 4 and the enhanced residual core brightness predicted by Model 2 relative to 1 and 3 .

Model $1(\longrightarrow)$

Mode1 $2(\ldots)$

Model $3(\mathrm{xxxx})$

Mode1 $4(-)$ 


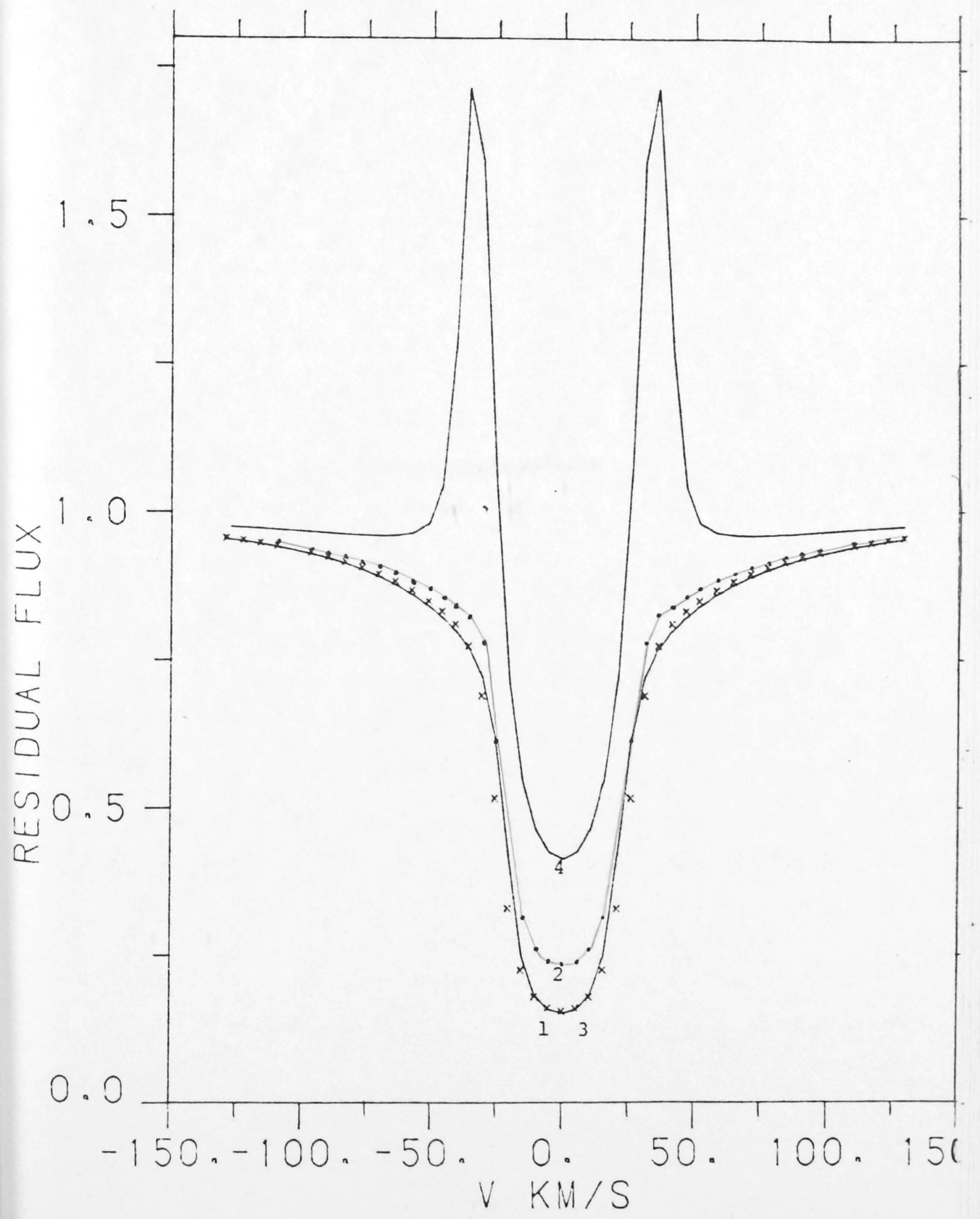




\section{FIGURE $5.3 \mathrm{~b}$}

Similar to Fig. 5.3a, except that comparisons are made between the computed $\mathrm{Ca}$ II $\mathrm{K}$ lines from the four chromosphere models. Note the broad Ca II K base emission widths predicted by the models with deeper lying temperature minima (Models 3 and 4). 


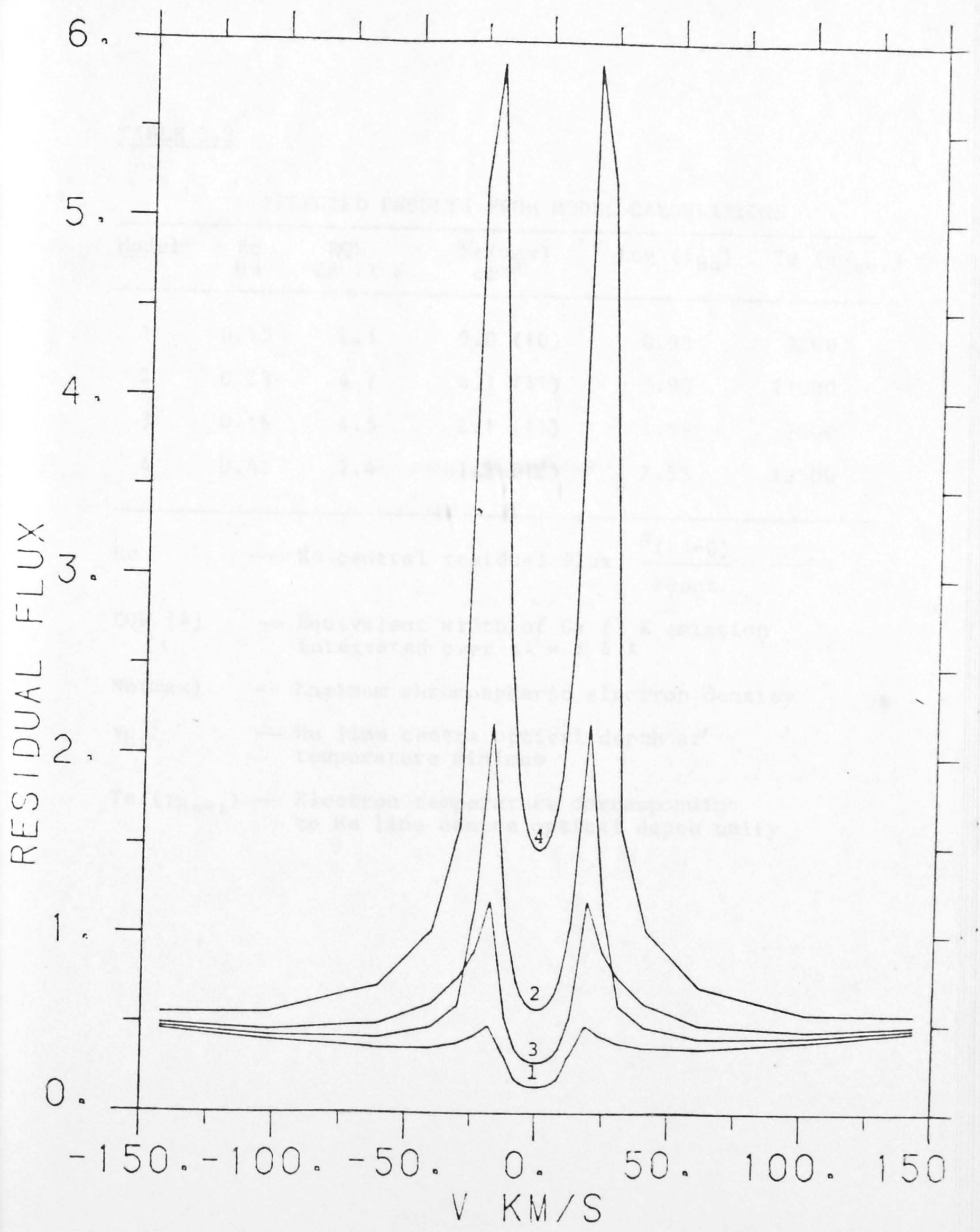


TABLE 5.2

SELECTED RESULTS FROM MODEL CALCULATIONS

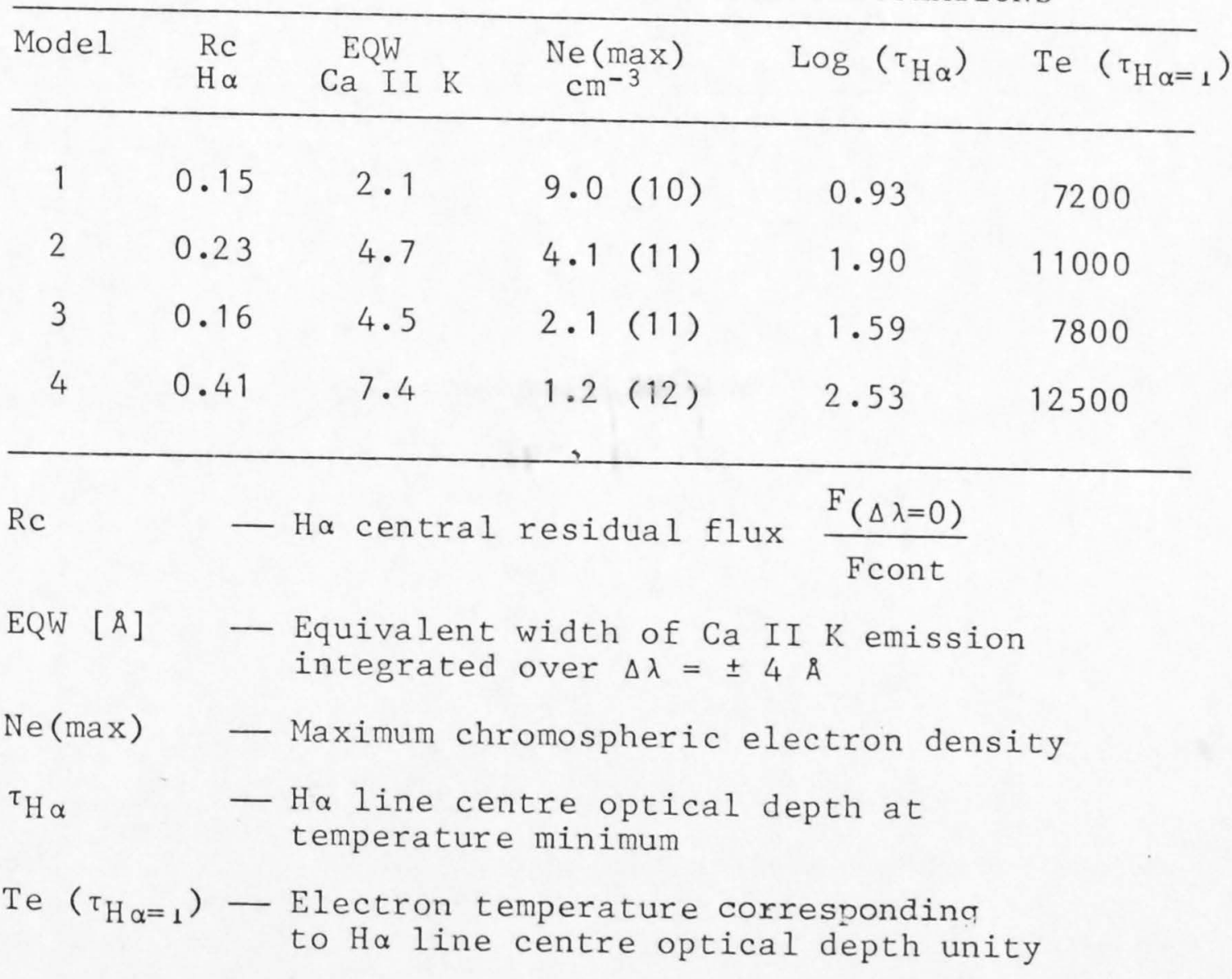


FIGURE 5.4

The distributions of $\log (\mathrm{Ne})$ with $\log (\mathrm{M})$ are illustrated for the chromosphere models. 


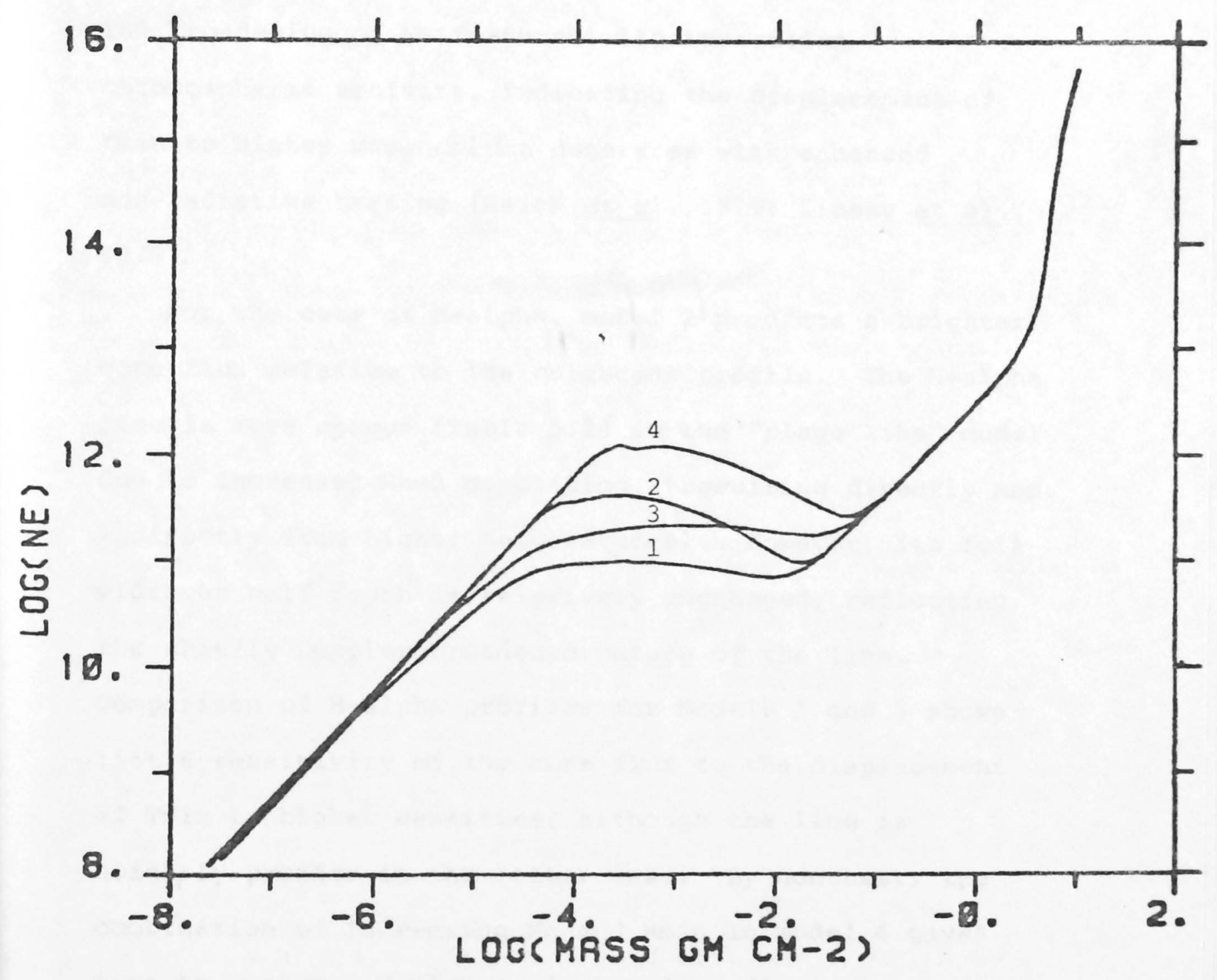


counterparts with higher lying temperature minima. This phenomenon can be understood in terms of the

Eddington-Barbier mapping (under CRD conditions) between the wavelength position of the damping controlled $\mathrm{Kl}$ feature and the location of Tmin (Ayres et al., 1975; Ayres, 1979). Measurements of $\mathrm{Wl}$ in a variety of active and quiet late type dwarfs have qualitatively confirmed the broadening of $\mathrm{Kl}$ features with increasing chromospheric activity, indicating the displacement of Tmin to higher mass column densities with enhanced non-radiative heating (Kelch et al.,1979; Linsky et al., 1979).

For the case of H-alpha, model 2 predicts a brighter core flux relative to the quiescent profile. The H-alpha line is more opaque (Table 5.2) in the "plage like" model due to increased $\mathrm{Hn}=2$ population, (resulting directly and indirectly from higher temperatures). However, its full width at half depth is relatively unchanged, reflecting the chiefly Doppler broadened nature of the line. Comparison of H-alpha profiles for models 1 and 3 shows little sensitivity of the core flux to the displacement of Tmin to higher densities, although the line is slightly broader in the latter case. By contrast, the combination of increasing Mo and Mmin in model 4 gives rise to a strong $\mathrm{H}$-alpha emission line displaying a deep central reversal.

Physical insight into these results can be obtained by considering the H-alpha frequency independent line source function, $\mathrm{S}_{\mathrm{H} \alpha}$. From the statistical equilibrium 
equations, $\mathrm{S}_{\mathrm{H} \alpha}$ can be formulated as (Thomas, 1957).

$$
S_{H \alpha}=\frac{\int J_{\nu} \phi_{\nu} d \nu+\varepsilon B \nu(T)+n B^{*}}{1+\varepsilon+\eta}
$$

where the first term in the numerator represents the scattering integral; $\phi_{\nu}$ is the normalised absorption profile and $J_{v}$ the mean intensity. The remaining terms denote source and sink parameters, which are discussed below. The distribution of $\log \left(\mathrm{S}_{\mathrm{H} \alpha}\right)$ with $\log (M)$ for each of the models is illustrated in Figure 5.5. Corresponding Planck functions and positions of $\mathrm{H}-\mathrm{al}$ pha line centre optical depth unity are shown for reference. In models 1 and $3, \mathrm{~S}_{\mathrm{H} \alpha}$ is uncoupled from the thermal structure in the chromosphere; and displays a decrease with height that is characteristic of a radiatively controlled line. Models 2 and 4 , on the other hand, demonstrate a clear coupling between $\mathrm{S}_{\mathrm{H} \alpha}$ and the increasing chromospheric Planck function and, most importantly, predict enhanced source functions at $\tau^{\mathrm{H} \alpha}=1$, relative to the shallow temperature gradient models.

The above coupling can be understood by examining the nature of the source/sink terms in equation 5.1. For analytic tractability, we approximate the H-alpha line as a resonance transition in a two bound level plus continuum $(k)$ model atom. This assumption is valid in regions where $\tau($ LYC $)>1 \emptyset$, where Ly-alpha opacity is sufficiently large to drive levels 1 and 2 into radiative detailed balance (Thomas, 1957). Following the analysis of Fosbury (1974), we ignore stimulated emissions and 


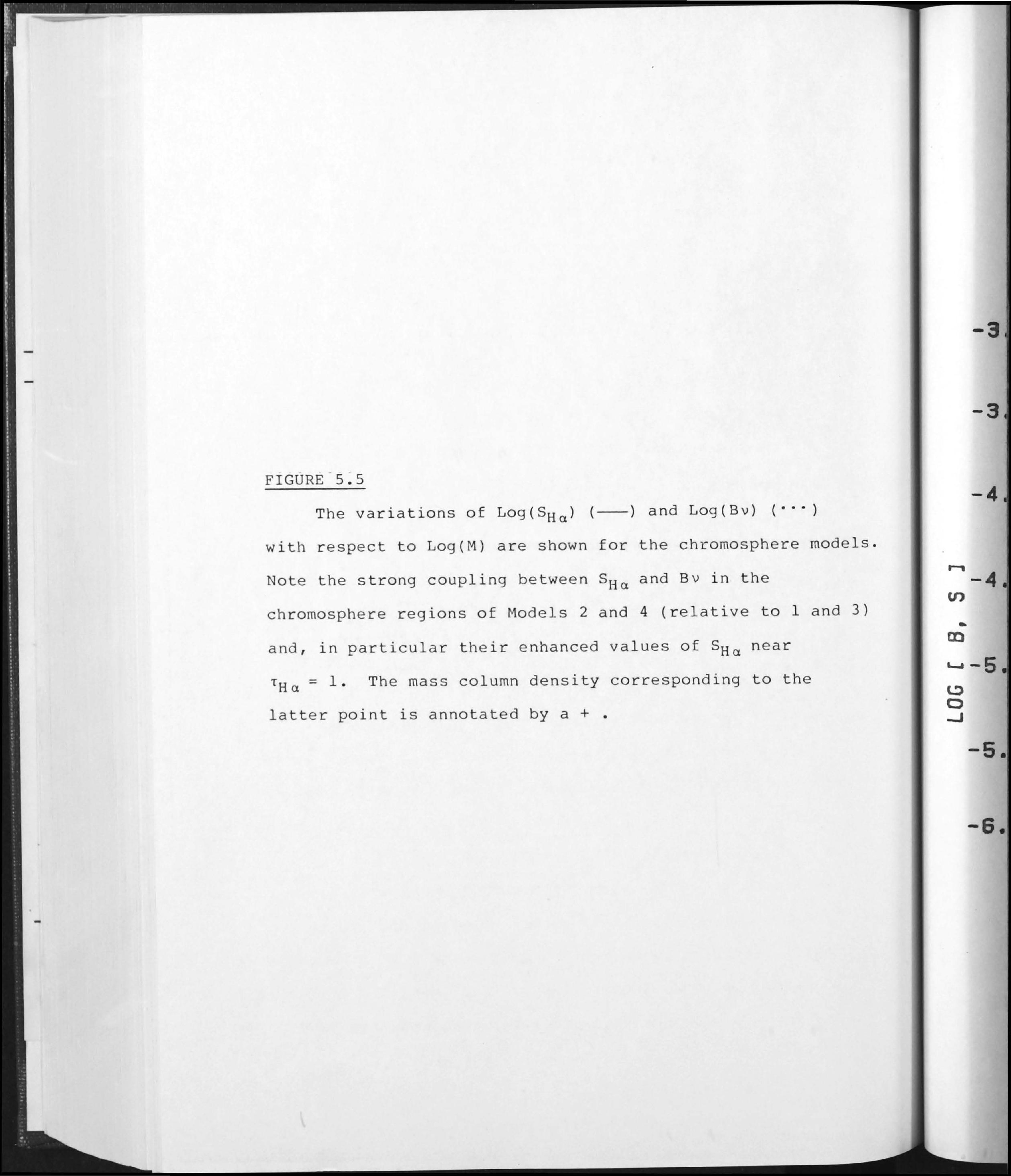




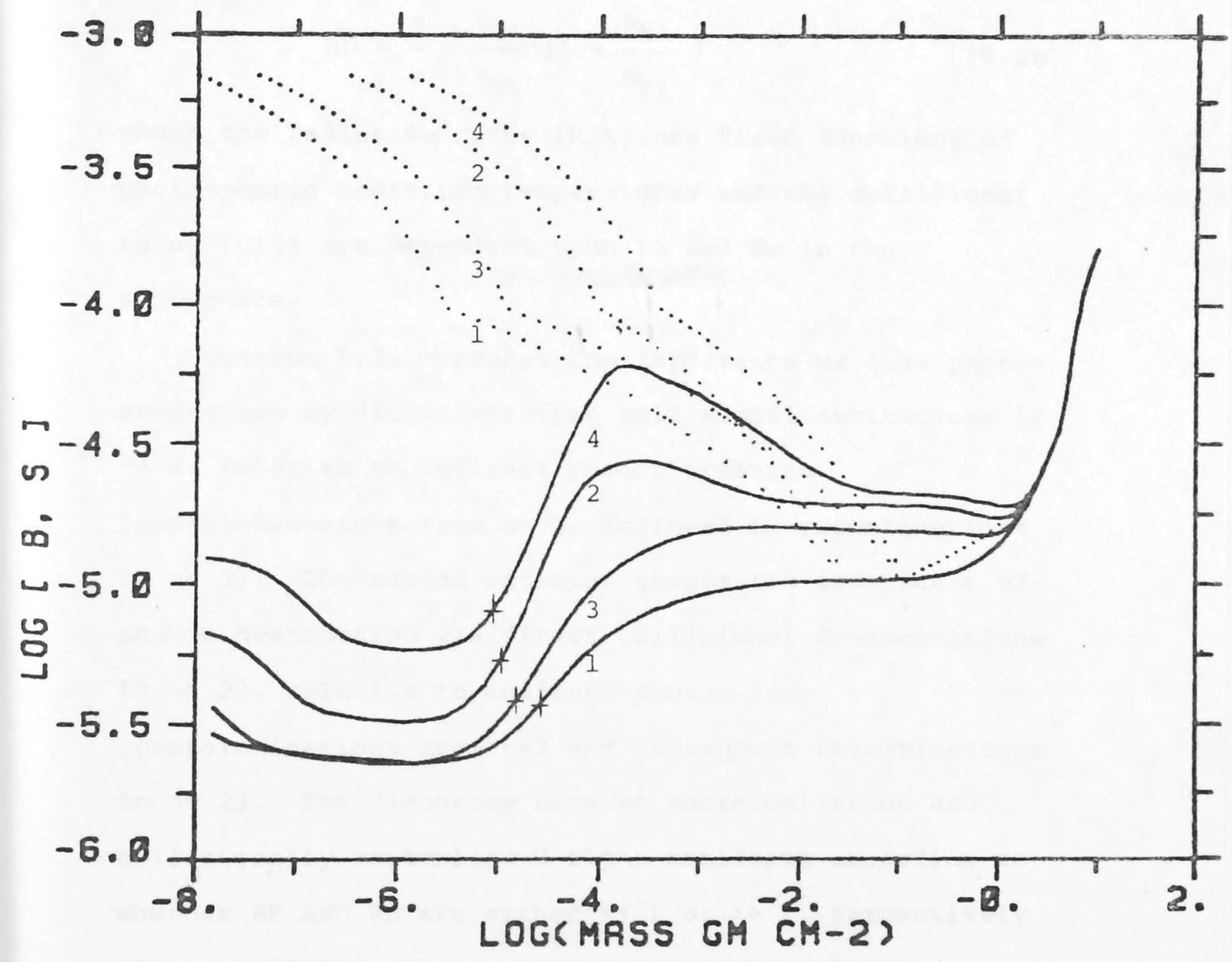


neglect collisional ionisation and recombination rates relative to their radiative counterparts. With these simplifications, the quantities in equation 5.1 can be expressed in terms of the ratios,

$$
\begin{aligned}
& R P=\frac{\varepsilon B \nu(T)}{n B^{*}}=\frac{C_{23}}{R_{2 k}}\left\{1+\frac{R_{k 2}}{R_{k 3}}\right\} \\
& R D=\frac{\varepsilon}{n}=\frac{C_{32}}{R_{3 k}}\left\{1+\frac{R_{k 3}}{R_{k 2}}\right\}
\end{aligned}
$$

where the radiative rates (Rik) are fixed functions of photospheric radiation temperatures and the collisional rates (Cij) are dependent upon $\mathrm{Te}$ and $\mathrm{Ne}$ in the atmosphere.

Equation 5.2 a measures the importance of line photon production by direct electron collisional excitations ( 2 $\rightarrow 3$ ) relative to indirect photon creation (photoionisations from $n=2$, followed by recombinations to $n=3)$. The second equation gauges the importance of photon destruction via direct collisional de-excitations $(3 \rightarrow 2)$, relative to indirect photon loss (photoionisations from $\mathrm{n}=3$ and subsequent recombinations to $n=2$ ). The dichotomy between photoionisation and collisionally controlled H-alpha manifests according to whether RP AND RD are either $<1$ or $\gg 1$, respectively (Thomas, 1957). Hence, by examining the relative behaviours of these ratios between each of the models, we can elucidate the degree to which the above competing processes influence the corresponding source functions. In Figures $5.6 \mathrm{a}$ and $\mathrm{b}$ we plot, respectively, $\log (\mathrm{RP})$ 
FIGURE $5.6 \mathrm{a}$

Annotated $\log (R P)-\log (M)$ distributions for each

chromosphere model. RP measures the importance of $\mathrm{H} \alpha$ photon production by direct, collisional relative to indirect, radiative processes. In the text we call attention to the lack of validity of our RP definition in regions where the Lyman lines are optically thin. 


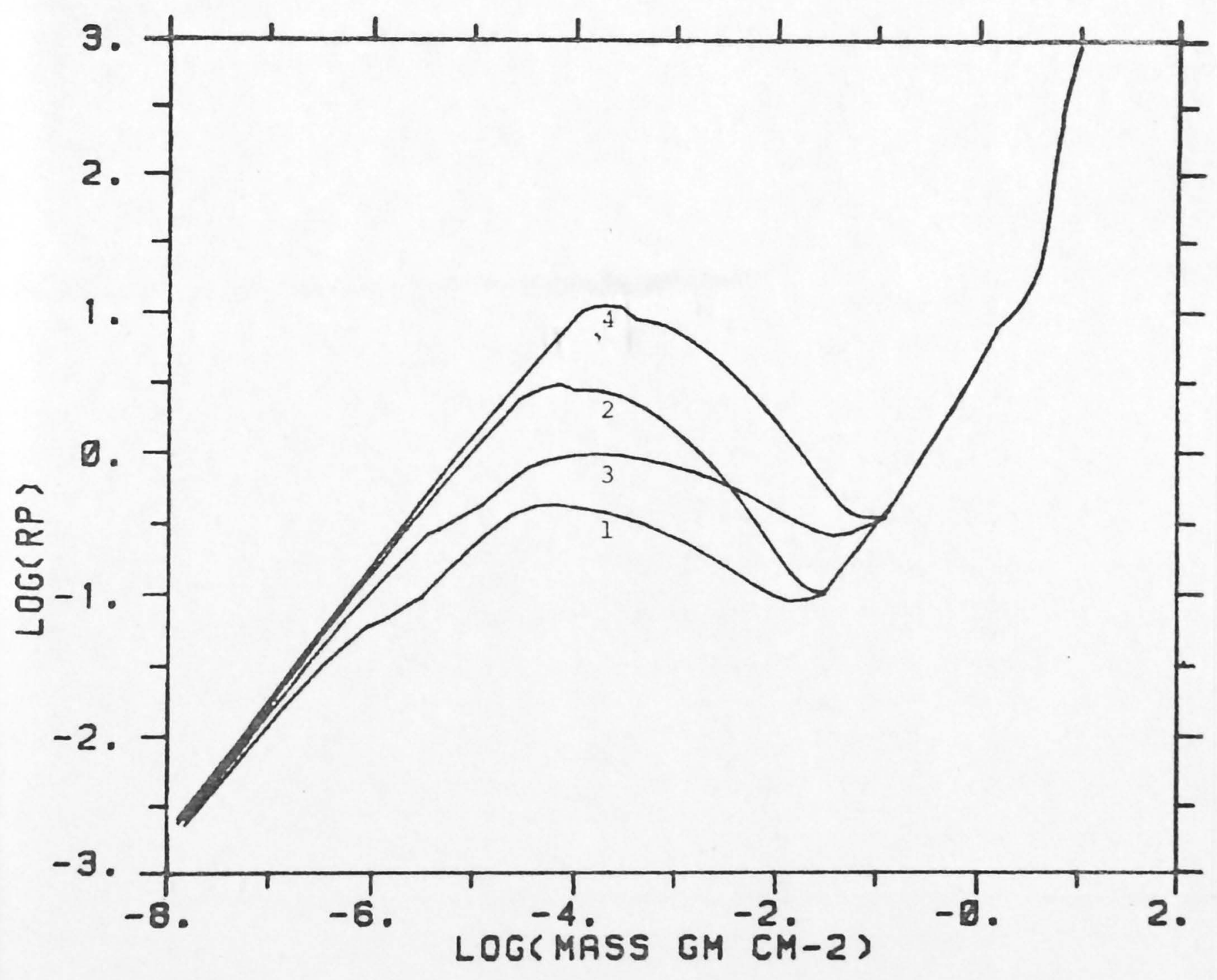


FIGURE $5.6 \mathrm{~b}$

Similar to Fig. 5.6a, except that the distribution of Log(RD) (the corresponding $\mathrm{H} \alpha$ direct/indirect sink ratio) with respect to $\log (M)$ is "shown for each model. 


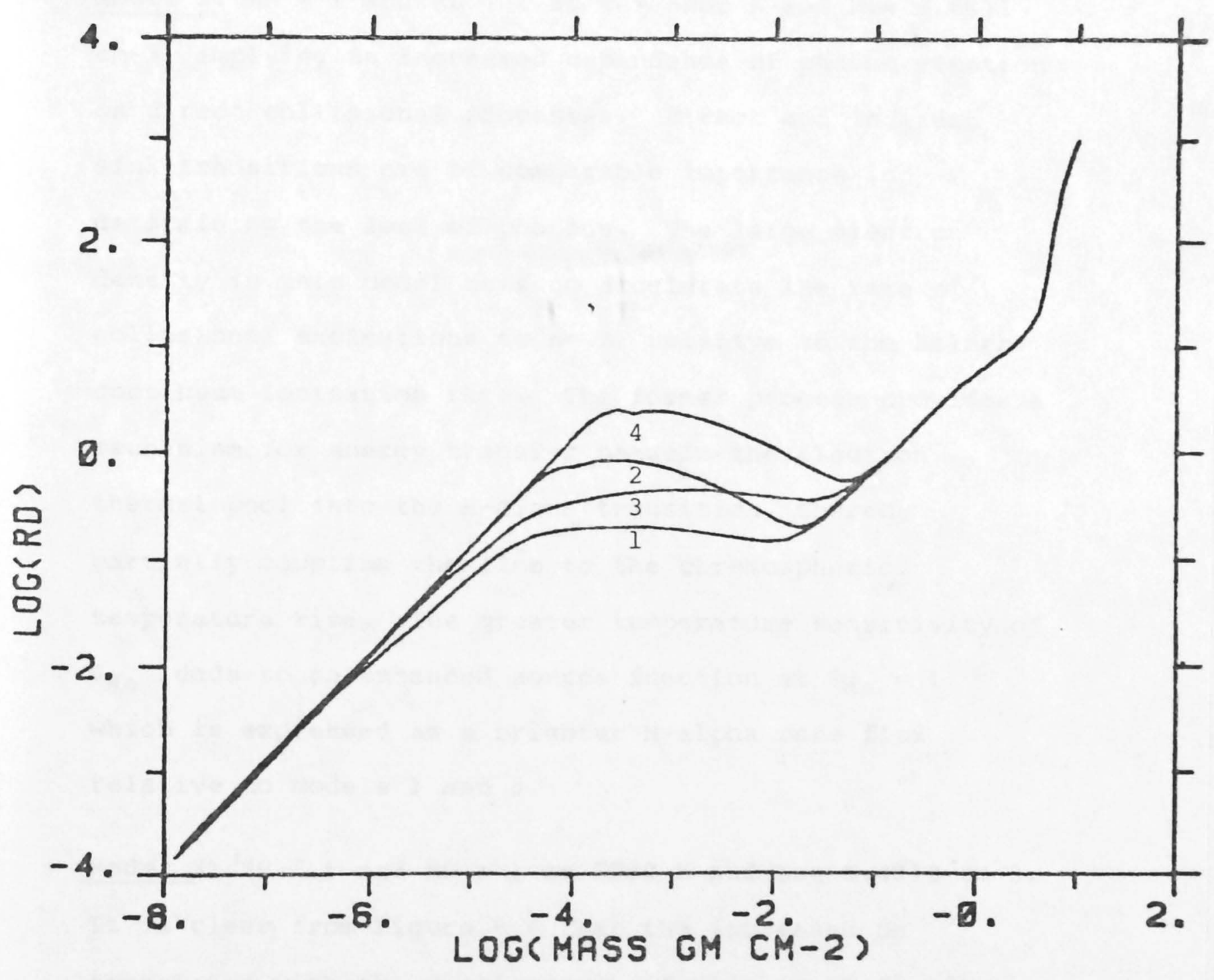


and $\log (R D)$ as functions of $\log (M)$ for the four chromosphere models.

Model I: RP $<1$ and RD $<1$ throughout the chromosphere, signifying the dominance of indirect radiative processes in controlling the production and destruction of line photons.

Model 2: RP $>1$ and $R D \sim 1$ at $T e=8000 \mathrm{~K}$ and $\mathrm{Ne}=3.6 \mathrm{E} 11$ $\mathrm{cm}-3$, implying an increased dependence of photon creation on direct collisional processes. Direct and indirect sink transitions are of comparable importance in determining the loss of photons. The large electron density in this model acts to accelerate the rate of collisional excitations to $n=3$, relative to the Balmer continuum ionisation rate. The former process provides a mechanism for energy transfer between the electron thermal pool into the H-alpha transition, thereby partially coupling the line to the chromospheric temperature rise. The greater temperature sensitivity of $\mathrm{S}_{\mathrm{H} \alpha}$ leads to an enhanced source function at $\tau_{\mathrm{H} \alpha}=1$, which is expressed as a brighter H-alpha core flux relative to models 1 and 3 .

Model 3: RP $\sim 1$ and $\mathrm{RD}<1$ at $8 \emptyset \emptyset \emptyset \mathrm{K}$ and $\mathrm{Ne}=5.4 \mathrm{El} \emptyset \mathrm{cm}-3$. It is clear from Figure 5.4 that the increased Ne associated with the displacement of Mmin is confined to the lower chromospheric layers. Towards higher layers, particularly where the H-alpha core is formed, Ne is a monotonically decreasing function of height. Although 
collisional and radiative processes are equally

competitive in their control of photon production, the relatively low Ne at $\tau_{\mathrm{H} \alpha}=1$ (compared to the previous case) is insufficient to activate a strong temperature sensitivity for $\mathrm{S}_{\mathrm{H} \alpha}$. The latter therefore demonstrates little coupling to the chromospheric temperature rise and $\mathrm{H}$-alpha appears in absorption.

Model 4: RP > 1 and $\mathrm{RD}>1$ at $8 \emptyset \emptyset \emptyset \mathrm{K}$ and $\mathrm{Ne}=1 \emptyset . \emptyset \mathrm{E} 12$ $\mathrm{cm}-3$. This extreme case gives rise to very high chromospheric Ne and a source function whose source and sink terms are dominated by collisionally induced transitions. The computed H-alpha profile is responsive to the chromospheric temperature rise and appears as a broad, optically thick emission line with a central reversal ascribed to non-LTE effects of the outer boundary (Athay, 1972).

The inclusion of collisional ionisation terms in our definitions of $\mathrm{RP}$ and $\mathrm{RD}$ will strengthen the temperature dependencies of these ratios (Gebbie and Steinitz, 1974) Above electron densities of $10 . \emptyset \mathrm{E} 12 \mathrm{~cm}-3$, collisional ionisations from level 3 will in fact begin to compete with corresponding photoionisations in determining the hydrogen statistical equlibrium.

\section{$\underline{5.4 \text { DISCUSSION }}$}

Albeit the H-alpha emission profile generated from model 4 bears no resemblance to that observed in single $G$ (or early K) active dwarf stars, it serves to illustrate 
the sensitivity of an otherwise radiatively dominated line to temperature-density variations that could occur in chromospheric regions subject to augmented energy release.

It is instructive to compare the results of model 4 with Balmer line transfer calculations by Cram and Mullan (1979), applied to flare phenomena in dMe stars. Based on schematic chromospheric (and transition region) temperature models, these authors demonstrated the potential usefulness of $\mathrm{H}-\mathrm{al}$ pha as a chromospheric indicator in such active stars. In particular, they showed that the combination of cool background radiation temperatures and steep chromospheric temperature rises resulted in a collisionally dominated H-alpha line, whose emission strength was particularly sensitive to the total amount of matter contained within the chromosphere and transition zone.

It is clear from our theoretical calculations that, even at the hotter effective temperatures pertinent to a G2 V star, a steep chromospheric temperature increase can affect H-alpha formation via the enhancement of collisional excitation processes.

From the results of model 4 it is apparent that too large an increase in $\mathrm{dT} / \mathrm{d} \log (M)$ and/or Mmin will conspire to produce $\mathrm{H}$-alpha (and Ca II K) in conflict with observations. In fact, the computed Ca II $\mathrm{K}$ line for this model is extremely strong compared to the brightest of the plage profiles calculated by Shine and Linsky (1974). It is in this sense that observations of 
will influence the H-alpha intensity by affecting the H-alpha optical depth scale. Changes in the latter can also be induced by variations in gas density and $\mathrm{Hn}=2$ excitation jevels (Basri et al., 1979), or by peculiarities in microvelocity fields. Presumably, these phenomena operate in addition to the H-alpha collisional coupling mechanism, however, the assessment of their relative importance in non-solar stars is beyond the scope of the present work.

Finally, we note that perturbations in the $\mathrm{T} z$ thermal structure can also play an important role in determining

\section{ERRATUM}

pages 93 and 94 are in reverse order.

consequences of temperature-ueworly

region on the formation of $\mathrm{H}-a l$ pha will be explored in more detail in Chapter Six.

\subsection{CONCLUSIONS}

The preliminary non-LTE transfer calculations we have conducted indicate that the parameterisation of enhanced chromospheric activity by steep chromospheric temperature structures, as invoked in the solar plage studies of Shine and Linsky (1974), is consistent with the H-alpha core brightening observed in active main sequence stars. On the basis of these computations, we conclude that 
will influence the H-alpha intensity by affecting the H-alpha optical depth scale. Changes in the latter can also be induced by variations in gas density and $\mathrm{Hn}=2$ excitation levels (Basri et al., 1979), or by peculiarities in microvelocity fields. Presumably, these phenomena operate in addition to the H-alpha collisional coupling mechanism, however, the assessment of their relative importance in non-solar stars is beyond the scope of the present work.

Finally, we note that perturbations in the $\mathrm{Tz}$ thermal structure can also play an important role in determining the behaviour of the H-alpha profile (Cram and Mullan, 1979; Cram and Woods, 1982). , Inspection of the source function distributions in Figure 5.5, particularly for models 2 and 4 , reveals that ${ }^{\tau} \mathrm{H} \alpha=1$ occurs in the $\mathrm{T} 2$ (see Table 5.2), and the H-alpha core flux is sensitive to the source function magnitude at this point. The consequences of temperature-density variations in this region on the formation of $\mathrm{H}-a l p h a$ will be explored in more detail in Chapter Six.

\subsection{CONCLUSIONS}

The preliminary non-LTE transfer calculations we have conducted indicate that the parameterisation of enhanced chromospheric activity by steep chromospheric temperature structures, as invoked in the solar plage studies of Shine and Linsky (1974), is consistent with the H-alpha core brightening observed in active main sequence stars. On the basis of these computations, we conclude that 
H-alpha, when utilised in conjunction with the Ca II (and

Mg II) resonance lines, will assist in constraining theoretical chromosphere models of late type active dwarfs.

The results of model 2 best illustrate the H-alpha core brightening with increasing Ca II $\mathrm{K}$ emission strength that is reflected in our observations. In order to ensure a strong temperature sensitivity for the H-alpha source function over the region where the line core is formed, one requires that large electron densities be sustained in these high temperature layers. This can be achieved by necessitating the existence of steep chromospheric temperature stuctures above the temperature minimum, although a simultaneous increase in Mmin is not ruled out by the results of our $\mathrm{H}-\mathrm{alpha}$ calculations. Clearly, displacing the chromosphere to larger densities, whilst maintaining a relatively shallow temperature gradient, does not fulfill the above requirement.

It has also been argued that the analogous "filling-in" of the H-alpha line core seen in filtergrams of the solar chromosphere (Athay, 1976) can be explained in terms of lateral changes in the shape of the line absorption profile $\left(\phi_{\nu}\right)$ in heterogenous regions of the atmosphere (Gebbie and Steinitz, 1973; Gebbie and Steinitz, 1974). A change in $\phi_{\nu}$ will modify $S_{H \alpha}$ through its control of the scattering integral in equation 5.1, independently of the chromospheric distribution of source and sink terms. Alternatively, local variations in $\phi_{v}$ 
electron collisional coupling between the H-alpha source function and a steep chromospheric temperature gradient offers a plausible explanation for this core brightening.

Although we have concentrated on $\mathrm{H}$-alpha formation in a G2 V star, the effects of collisional processes will be more pronounced in the active chromospheres of cooler dwarfs, since the associated reduction in effective temperatures acts to decrease the relative importance of indirect to direct processes in influencing the behaviour of H-alpha (Fosbury, 1974).

By analogy with solar active regions, we presume that the steep chromospheric temperature structures are consequences of amplified non-radiative heating in regions pervaded by magnetic fields. Evidence for the existence of magnetic active regions, like solar plages, on the cool dwarfs Epsilon Eri and Xi Boo (A) is given by Linsky et al. (1982). Their studies demonstrate that stellar chromospheric and high temperature emission line (C IV, S IV, N V) surface fluxes for these active stars is similar to that in solar plages. The detection of magnetic fields on Xi Boo (A) and 70 Oph (A) by Robinson et al. (1980) further reinforces the possibility of magnetic active regions on their surfaces.

The observations presented in section 5.1 and Chapter Three provide additional evidence in favour of the stellar plage hypothesis since the enhanced $\mathrm{H}$-alpha core brightness among active dwarfs in the sample parallels the well known contrasts seen in H-alpha filtergrams of the solar surface. In particular, the brightening of the 
H-alpha core indicates that it is formed in regions of higher temperature and density than the quiescent sun chromosphere, precisely the important attribute of magnetic active regions revealed in the Ca II $K$ modelling of plages by Shine and Linsky (1974).

Accordingly, an extension of the previous $\mathrm{Ca}$ II work to detailed numerical simulation studies of $\mathrm{H}-a l p h a$ in broad range of quiet and active dwarfs, in the spirit of the preliminary modelling of Kelch et al. (1979) should provide an increased understanding of the temperature density profiles of magnetic active regions, the surface coverage of such regions and, perhaps, some insight into the elusive chromospheric heating mechanisms. 
CHAPTER SIX

\section{THE H-ALPHA PROFILE IN 70 OPHIUCHI A (KO V) \\ $\underline{\text { AND ARCTURUS (K2 III) }}$}

\section{$\underline{6.1 \text { INTRODUCTION }}$}

The preliminary transfer calculations performed in the previous chapter have highlighted the potential usefulness of the H-alpha absorption profile as a constraining observational parameter in the determination of stellar model chromospheres. The present investigation endeavours to establish further this capability by examining the degree to which chromosphere models, inferred from the Ca II and Mg II resonance lines, produce H-alpha profiles consistent with observations. We adopt, as case studies, the two stars 70 Oph A (KO V) and Arcturus (K2 III) for which chromospheric temperature and microturbulence distributions have been well determined from observations of the latter lines.

The formation of the subordinate H-alpha line has been shown to depend on details of the stellar chromosphere in a slightly different manner from the resonance lines (cf. Jefferies and Thomas, 1958). Consequently, H-alpha profile synthesis could provide a consistency check for model chromospheres deduced from the more traditional $\mathrm{Ca}$ II and Mg II diagnostics. 
Moderate success in obtaining self-consistency between $\mathrm{H}$-alpha and $\mathrm{Ca}$ II $\mathrm{K}$ is exemplified by the work of Kelch et al. (1979). Their H-alpha calculations for the cool dwarfs 61 Cyg $B($ dMO) and EQ Vir (dK7e), using models derived from Ca II $K$ partial redistribution (PRD) analyses, predicted $\mathrm{H}$-alpha absorption from the former star and emission from the latter in qualitative agreement with observations. Based on similar model calculations for the dwarfs GL 411 (dM2) and EQ Vir, Giampapa et al. (1982) demonstrated that, as a consequence of the differing regions over which $\mathrm{H}-\mathrm{al}$ pha and $\mathrm{Ca} I \mathrm{I} \mathrm{K}$ are formed, it is possible to construct single component, homogenous model chromospheres for $M$ dwarfs that match simultaneously observations of these 1 ines.

In the light of the above work, we analyse the $\mathrm{H}-\mathrm{alpha}$ and $\mathrm{Ca}$ II $\mathrm{K}$ profiles in $70 \mathrm{Oph} \mathrm{A}$ and Arcturus from the point of view of addressing whether single component, homogenous models form adequate representations for their respective chromospheres. These two stars provide interesting and contrasting cases upon which to base this investigation. $70 \mathrm{Oph} A$ is a well known active KO dwarf whose upper photospheric and chromospheric structure has been analysed by Kelch (1978). Its mean chromospheric electron density is quite high $(\mathrm{Ne} \sim 1.0 \mathrm{Ell} \mathrm{cm}-3$ ) indicating that electron collisional processes may be important in affecting the sensitivity of its H-alpha profile to chromospheric thermal structure (cf. discussion, Chapter Five). On the 
other hand, Arcturus is an archetype red giant (K2 III), metal deficient and of moderate chromospheric activity relative to $70 \mathrm{Oph} A$ and the mean quiet sun (Ayres and Linsky, 1975b). Owing to much lower electron densities ( $\mathrm{Ne}<1.0 \mathrm{Ell} \mathrm{cm}-3$ ), its H-alpha profile is essentially radiatively controlled and, consequently, is expected to behave quite differently than in 70 Oph A (Fosbury, 1974 ).

\subsection{OPHIUCHI A CHROMOSPHERE MODELS}

\subsubsection{Description of Models}

Figure 6.1 illustrates the electron temperature-mass distribution derived by Kelch (1978) to yield reasonable agreement with spatially averaged emission fluxes in the $\mathrm{Ca}$ II and Mg II lines. We adopt this "best-fit" model (denoted model 1 ) as our reference chromosphere for 70 Oph A and consider three variations of its upper chromospheric and transition zone (TZ) structure (depicted in Figure 6.1). Defining characteristics for each of these models are compiled in Table 6.1 and are discussed below.

Principle features of the Kelch (1978) model include:

(i) a lower photospheric segment adapted from a line blanketed, radiative equilibrium atmosphere (Kurucz, 1974) with $\operatorname{Teff} / \log (\mathrm{g}) /[\mathrm{Fe} / \mathrm{H}]=5150 / 4.4 / 0.0$, and $\mathrm{a}$ constant microturbulence (Vturb) of $2 \mathrm{~km} / \mathrm{s}$;

(ii) a lower chromospheric temperature rise, linear in 


\section{FIGURE 6.1}

Electron temperature-mass column density distributions for the four 70 Oph A chromosphere models considered in this investigation. Model 1 corresponds to model A of Kelch (1978), which was inferred from PRD calculations of Mg II and $\mathrm{Ca}$ II resonance $\mathrm{lines.} \mathrm{See} \mathrm{text} \mathrm{for} \mathrm{details} \mathrm{of}$ remaining models. 


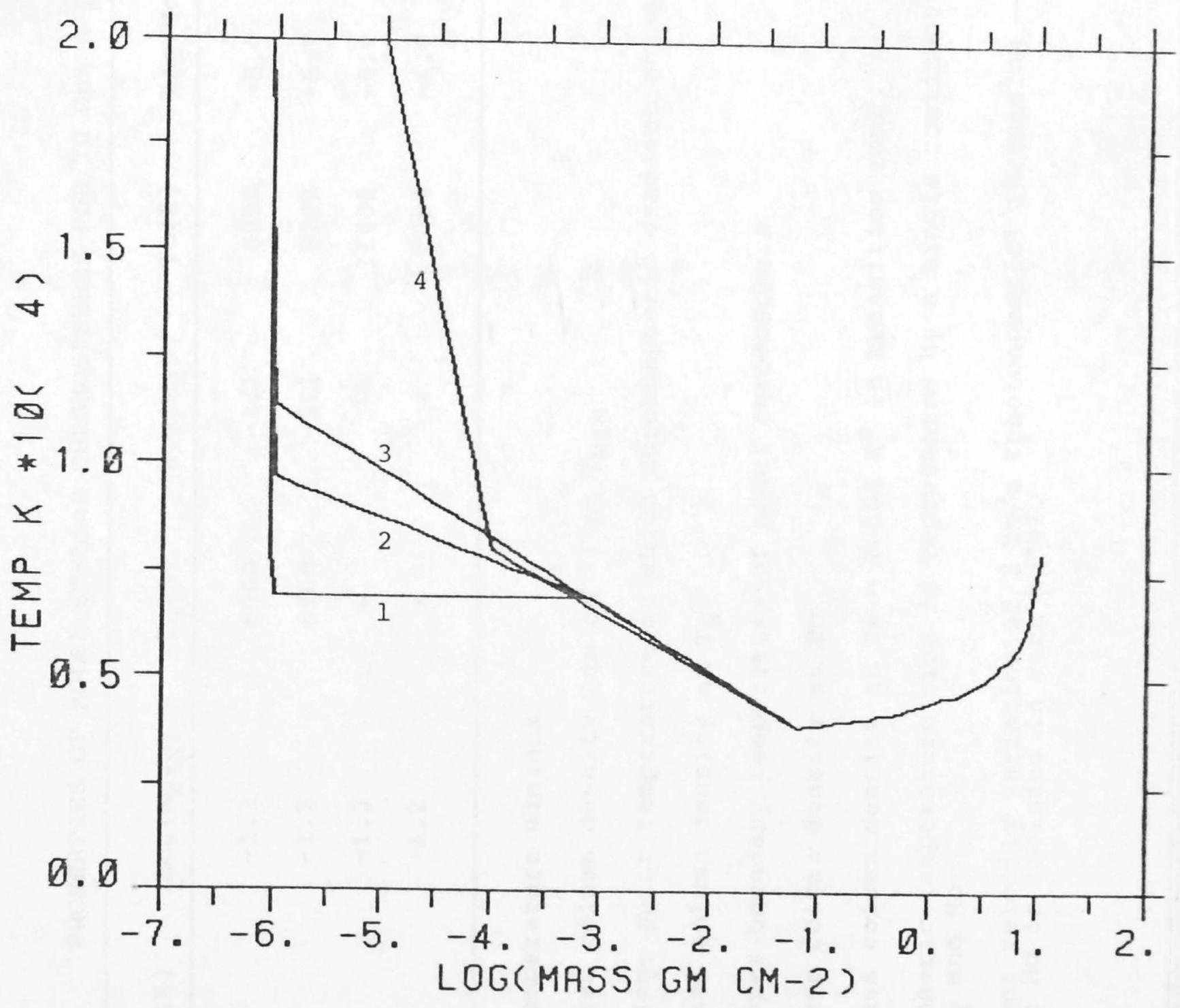


TABLE 6.1

PROPERTIES OF MODEL STELLAR CHROMOSPHERES FOR 70 Oph A (KO V)

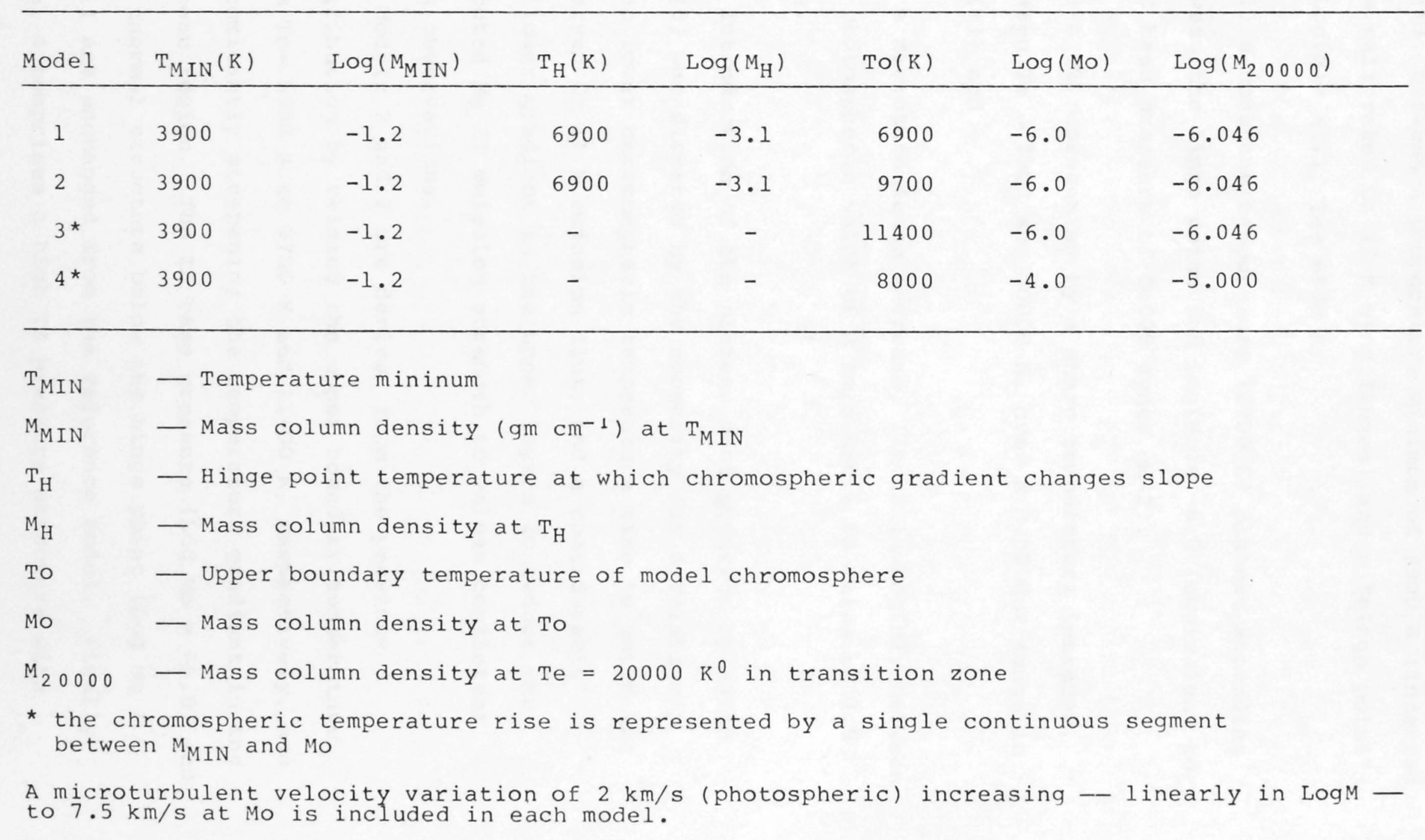


Log(M), between a temperature minimum of $3900 \mathrm{~K}$ (inferred from calibrated $\mathrm{Ca}$ II $K$ wing fluxes) and a "hinge point" at $\log (M)=-3.1, T e=6900 \mathrm{~K}$;

(iii) a constant temperature (6900 K) plateau extending between the hinge point and $\log \left(\mathrm{MO}_{\mathrm{O}}\right)=-6.0$ (equivalent to a TZ base pressure of 0.025 dynes $\mathrm{cm}-2$ ).

(iv) A TZ represented by a sharp temperature increase, between $\mathrm{Te} \backsim 7000$ and $20000 \mathrm{~K}$, over a 0.05 dex range in $\log (M)$; and

(v) a microturbulence increase, linear in $\log (M)$, between the photospheric value of $2 \mathrm{~km} / \mathrm{s}$ and a Tz value of 7.5 $\mathrm{km} / \mathrm{s}$.

Introduction of the plateau configuration by Kelch (1978) was dictated by the necessity for a relatively steep lower chromospheric temperature rise to match the observed Ca II K emission flux, and a considerably shallower gradient in the upper layers to reduce the computed Mg II emission strength to values consistent with observations.

Models 2 and 3 are derived from the previous distribution by raising the upper boundary temperature from $\mathrm{To}=6900 \mathrm{~K}$ to $9700 \mathrm{~K}$ and $11400 \mathrm{~K}$, respectively, and concomitantly steepening the temperature gradient in the plateau region. The $\mathrm{Tz}$ base pressure ( Log $M O=-6.0$ ) and the thermal structure below the hinge point (Log Mo > -3.1) are unchanged from the reference model. Finally, model 4 comprises a high Tz pressure structure with 
$\log (M O)=-4.0$ and a single steep chromospheric temperature rise between $\mathrm{Tmin}$ and $\mathrm{To}=8000 \mathrm{~K}$.

\subsubsection{Model Calculations And Results}

We use the non-LTE profile synthesis code (see Chapter Four) to solve for the H-alpha line from the pre-specified chromosphere models. The Lyman alpha, beta and continuum transitions are treated explicitly in the radiative transfer solution, while fixed rate transitions (Chapter Four, section 4.7.1) are specified by radiation temperatures (Trad) scaled in effective temperature from the solar values of Ayres and Linsky (1975a). The sensitivity of the computed $\mathrm{H}$-alpha line to errors in these background temperatures is investigated below.

Figure 6.2 illustrates the depth dependence of electron densities (Ne) obtained from the coupled solution of the hydrostatic equilibrium and hydrogen transfer equations. These densities, together with the converged hydrogen populations, are used in a transfer solution for the $\mathrm{Ca}$ II H, K and IR triplet lines, assuming a solar calcium abundance (Kelch, 1978). Fixed continuum transitions are incorporated using radiation temperatures set equal to the effective temperature of 70 Oph A. The Ca II lines are computed under the assumption of complete redistribution (CRD). Consequently, the profiles may be less accurate - particularly in the $\mathrm{K}$ damping wings - compared to more refined PRD calculations (Shine et al.., 1975; Kelch, 1978). Nevertheless, they are useful in examinations of first order differential 


\section{FIGURE 6.2}

The distribution of $\log (\mathrm{Ne})$ against $\log (M)$ is

illustrated for models 1 to 3 (solid lines) and 4 (dotted line). 


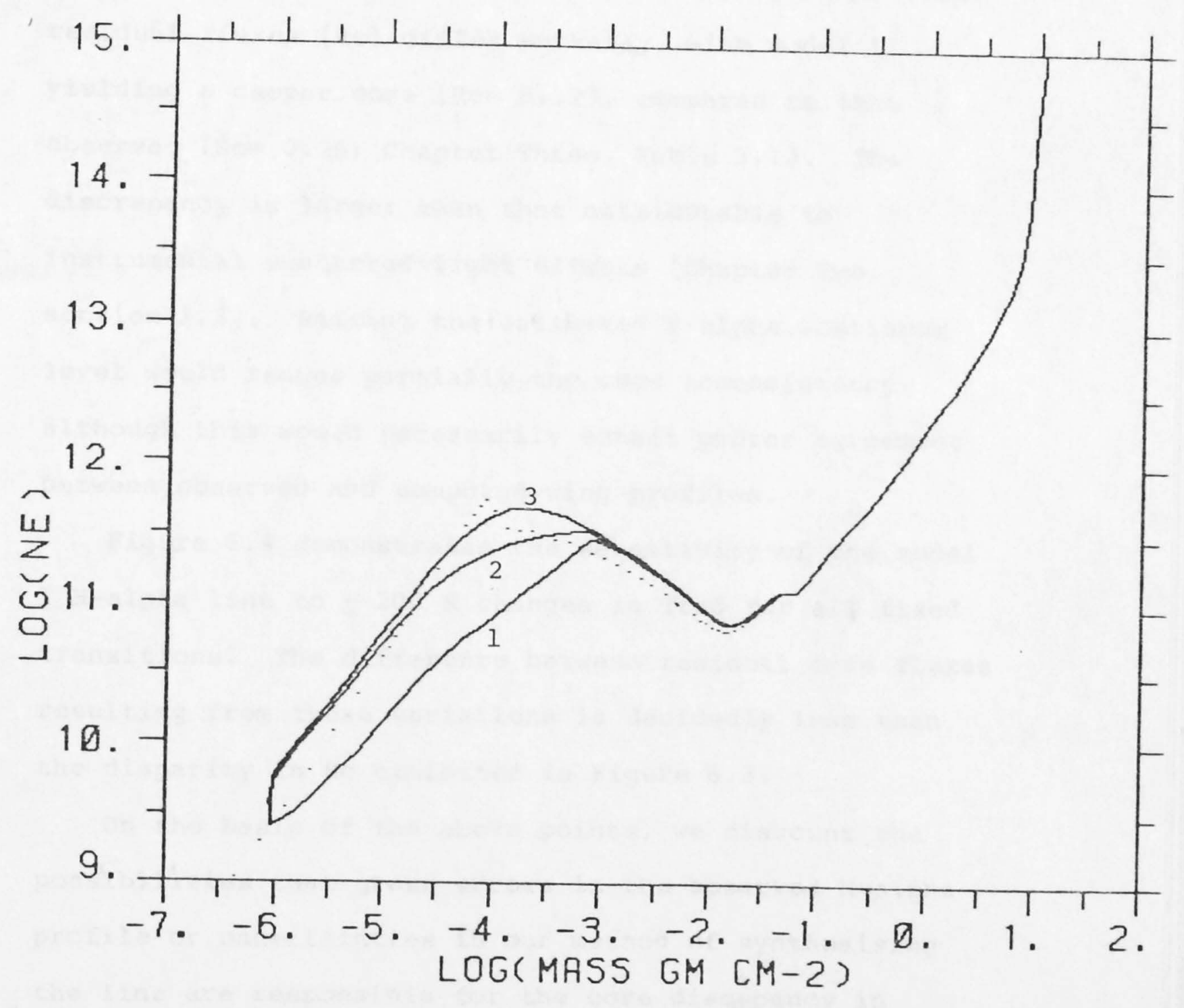


effects resulting from the various temperature models.

\section{(a) Examination of $\mathrm{H}$-alpha profiles}

Figure 6.3 compares the H-alpha line from model 1 with our observed 70 Oph A profile (atlas, Appendix 2.1). Theoretical and empirical H-alpha full widths at half depth compare quite favorably. On the other hand, core residual fluxes (RC) differ markedly, with model I yielding a deeper core $\left(\mathrm{RC}_{\mathrm{C}}=0.12\right)$, compared to that observed ( $R c=0.26$; Chapter Three, Table 3.1). The discrepancy is larger than that attributable to instrumental scattered light effects (Chapter Two, section 2.3). Raising the estimated H-alpha continuum level would reduce partially the core inconsistency, although this would necessarily entail poorer agreement between observed and computed wing profiles.

Figure 6.4 demonstrates the sensitivity of the model $1 \mathrm{H}$-alpha line to $\pm 200 \mathrm{~K}$ changes in Trad for all fixed transitions. The difference between residual core fluxes resulting from these variations is decidedly less than the disparity in Rc exhibited in Figure 6.3 .

On the basis of the above points, we discount the possibilities that gross errors in the observed H-alpha profile or uncertainties in our method of synthesising the line are responsible for the core disç्र epancy in Figure 6.3. Rather, we argue that the inconsistency is related to an inadequacy of the model 1 chromosphere structure, particularly in the region where the $\mathrm{H}-a l$ pha line core is formed. 
FIGURE 6.3

A comparison between the computed $\mathrm{H}$-alpha line of model 1 with the observed 70 oph A profile (on common velocity and continuum flux scales). 


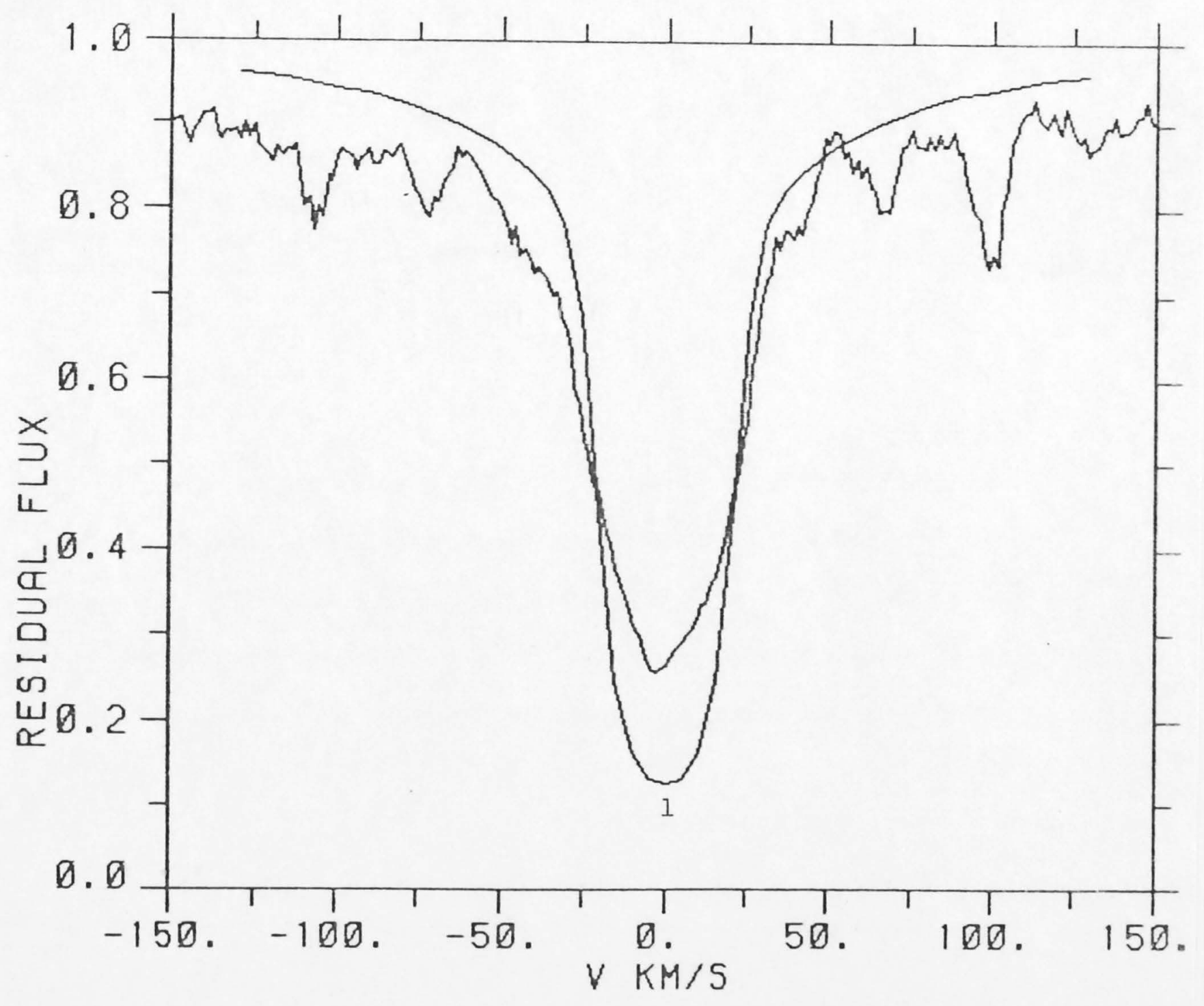




\section{FIGURE 6.4}

The $\mathrm{H} \alpha$ line from model $1(-)$ is compared with profiles computed using background radiation temperatures increased by $200 \mathrm{~K}^{0}(\mathrm{x} \times \mathrm{xx})$ and decreased by $200 \mathrm{~K}^{0}(\ldots)$, relative to those of model 1 . The differences in the line core resulting from these perturbations are qenerally small. 


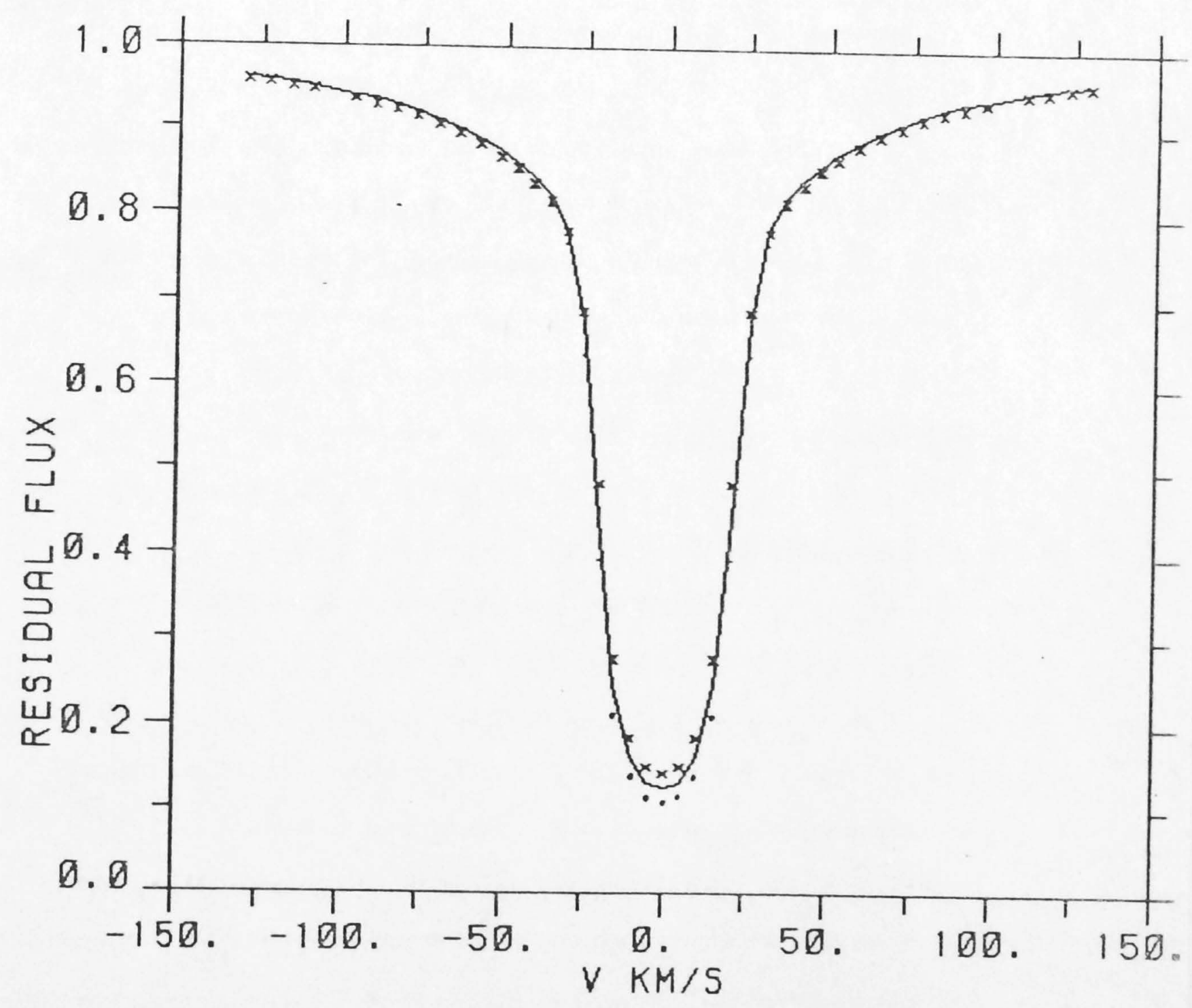


Inspection of the H-alpha optical depth scales for model 1 reveals that line centre optical depth unity ${ }^{(}{ }^{\tau} \mathrm{H} \alpha$ =1) occurs precisely in the plateau region at $\log (M)=$ -4.5 and $\mathrm{Ne}=4.0 \mathrm{E} 10 \mathrm{~cm}-3$. Therefore, based on the arguments developed in the previous chapter, we conjecture that the enhanced Rc in the observed 70 Oph A profile is indicative of a hot upper chromospheric component, with an electron temperature above the $6900 \mathrm{~K}$ plateau inferred from Ca II and Mg II observations.

To test this hypothesis, we investigate the responses of the H-alpha line to adhoc temperature enhancements in the plateau region as represented in models 2 and 3 . The resultant profiles are compared with observation in Figure 6.5. Evidently, steepening the upper chromospheric temperature gradient by increasing To (with fixed Mo) can lead to a "filling-in" of the H-alpha core. The H-alpha profile of model 3 maps closely the observed 70 Oph A core; however its wings are discrepant in the appearance of emission components at $\Delta \lambda=0.6 \AA$.

The fact that H-alpha wing emissions are not observed in 70 Oph A may mitigate against the validity of such enhanced To models. For the case of solar Ca II K features, it has been argued that large scale surface turbulent motions (macroturbulence) can play an important role in removing the characteristic Ca II K self-reversal feature predicted by optically thick, static chromosphere models (Shine and Linsky, 1974; Durrant et al., 1976;

Kneer and Mattig, 1979). Presumably, similar dynamical processes operating on 70 Oph A may be involved in 
FIGURE 6.5

A comparison between the $\mathrm{H}$-alpha $\mathrm{l}$ ines computed from models $1(-), 2(\ldots)$ and $3(x \times x x)$ with the observed 70 Oph A profile. Note the enhancement in Rc with increasing temperature gradient in the plateau region of model 1 . The H-alpha core profile of model 3 maps closely with that observed. The corresponding wing profile is, however, discrepant in the appearance of emission components at $\Delta \lambda=$ $0.6 \AA$. 


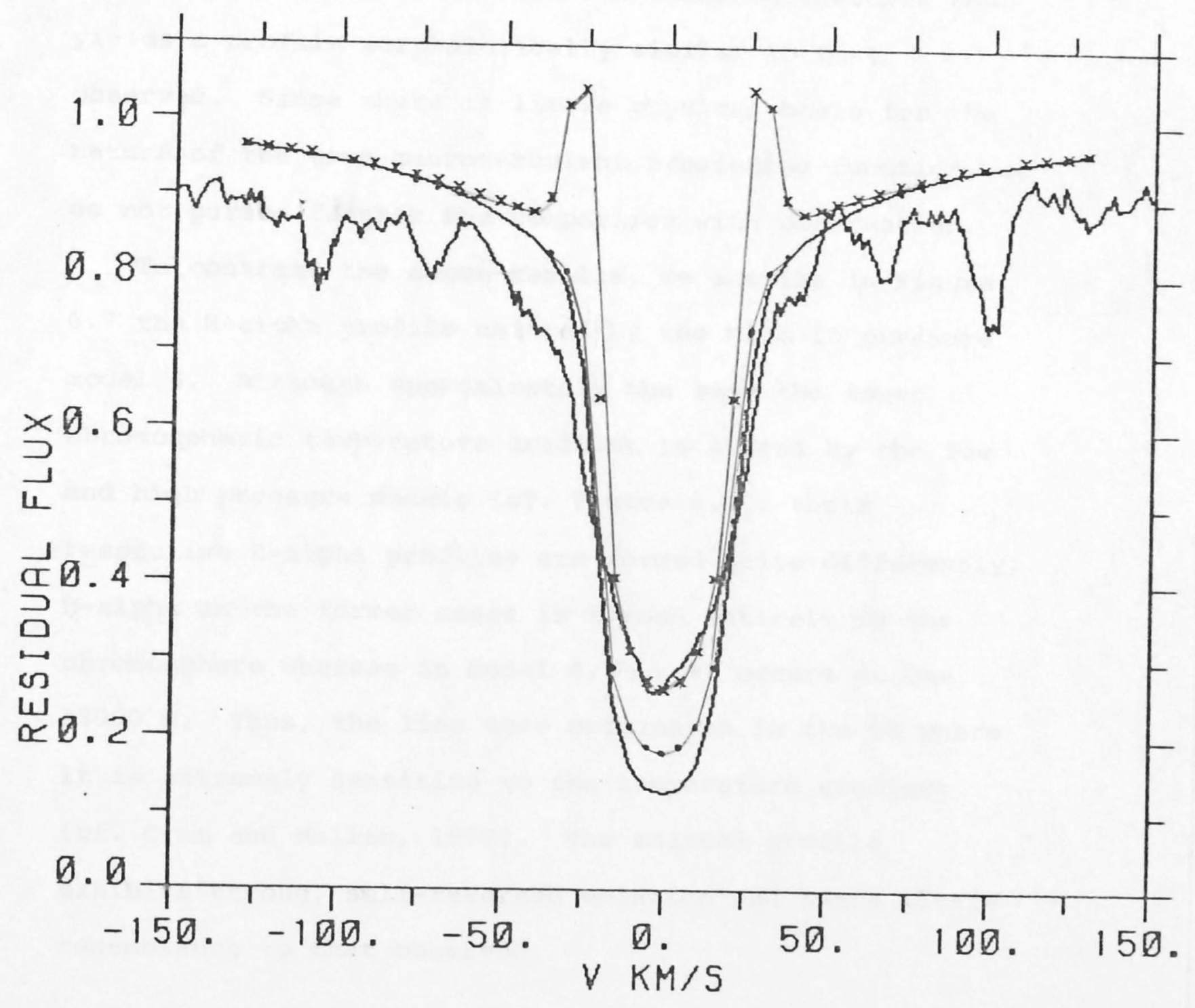


smearing the presence of weak H-alpha emission structures.

To illustrate the likely first order effects of such macroturbulent motions on the H-alpha profile of model 3 , we convolve it with a gaussian function of arbitrarily chosen FWHM $25 \mathrm{~km} / \mathrm{s}$. As shown in Figure 6.6, this process completely attenuates the emission features and yields a profile morphologically similar to that observed. Since there is little physical basis for the nature of the true macroturbulent broadening function, we do not pursue further the comparison with observation.

To contrast the above results, we examine in Figure 6.7 the H-alpha profile emitted by the high Tz pressure model 4. Although approximately the same the lower chromospheric temperature gradient is shared by the low and high pressure models (cf. Figure 6.1), their respective H-alpha profiles are formed quite differently. $\mathrm{H}$-alpha in the former cases is formed entirely in the chromosphere whereas in model $4, \tau_{\mathrm{H} \alpha}=1$ occurs at $\mathrm{Te}=$ $15000 \mathrm{~K}$. Thus, the line core originates in the $\mathrm{TZ}$ where it is extremely sensitive to the temperature gradient (cf. Cram and Mullan, 1979). The emitted profile exhibits strong, self-reversed emission and bears little resemblance to that observed.

\section{(b) Behaviour of $\mathrm{H}$-alpha source functions}

Insight into the above results can be gained by studying the the $\mathrm{H}-\mathrm{al}$ pha line source function $\left(\mathrm{S}_{\mathrm{H} \alpha}\right)$ in the various models. The distributions of $\mathrm{S}_{\mathrm{H} \alpha \text { for the low }}$ 


\section{FIGURE 6.6}

The observed 70 Oph A H-alpha profile is compared with the model 3 profile smeared by a $25 \mathrm{~km} / \mathrm{s}$ width gaussian function. Note the attenuation of the wing emission components and the more favorable fit to the observed line wings (cf. Fig. 6.5). Although the choice of gaussian width is unrealistically large, the above comparison illustrates the likely consequences of random atmospheric motions on the behaviour of spectral lines, such as H-alpha. 


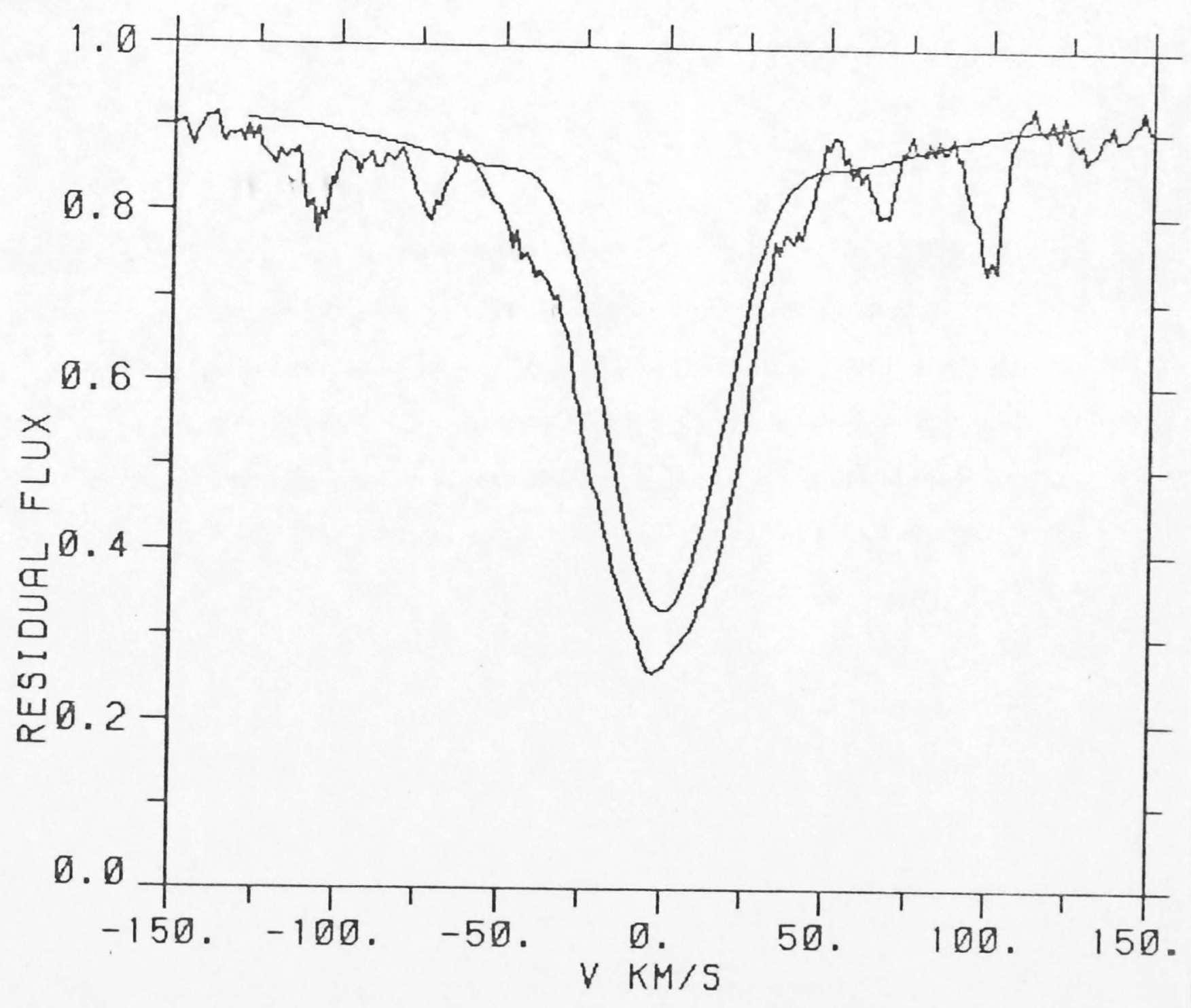




\section{FIGURE 6.7}

H-alpha profiles from models 1 (low TZ pressure) and 4 (high TZ pressure) are compared with the observed 70 Oph A profile. Wing emission features and a bright central intensity are predicted by the high pressure model 4 . 


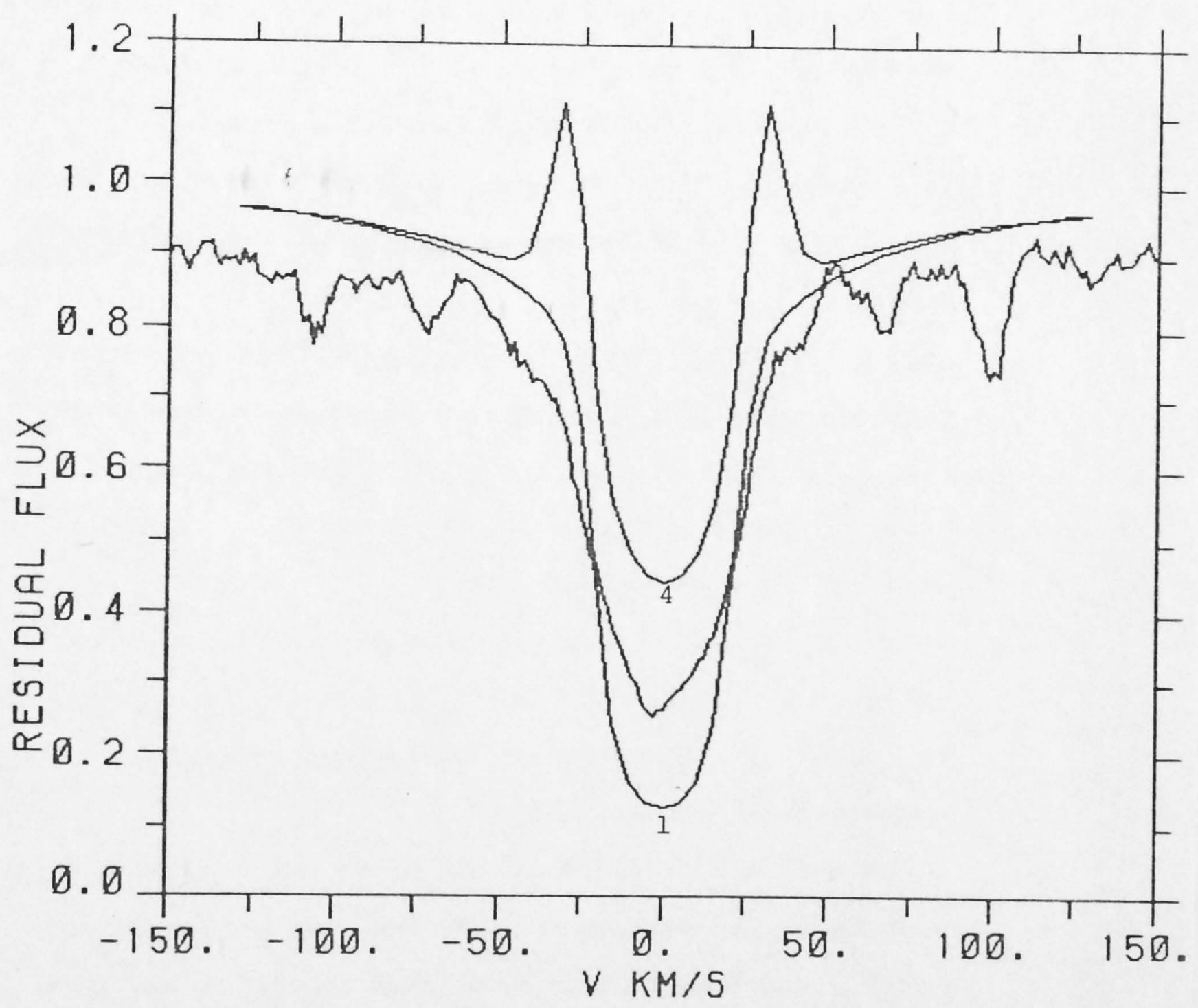


pressure models are plotted in Figure 6.8, together with the corresponding variations in $\mathrm{B}(\mathrm{T})$. The positions corresponding to H-alpha optical depth unity at line centre and at $\Delta \lambda=0.6 \AA$ (emission wavelength) are marked for reference. We note that in the temperature minimum region of each model, $\mathrm{S}_{\mathrm{H} \alpha}$ is uncoupled from $\mathrm{B}(\mathrm{T})$. This situation is a consequence of these layers being optically thin, such that the scattering term in $\mathrm{S}_{\mathrm{H} \alpha \text { is }}$ controlled completely by radiation emanating upwards from the photosphere and directed downwards from the chromosphere. Thus, the H-alpha profile provides little information on the thermal structure in these lower layers .

In the upper chromosphere of model $1, \mathrm{~S}_{\mathrm{H} \alpha}$ exhibits a very weak coupling with the chromospheric temperature rise. By contrast, the source functions in models 2 and 3 show pronounced maxima in the range $-4.0<\log (M)<$ -3.0 and enhanced values at ${ }^{\top} H_{\alpha}=1$. This source function behaviour can be understood through consideration of the source and sink terms which determine the sensitivity of $\mathrm{S}_{\mathrm{H} \alpha}$ upon local electron and background radiation temperatures (Thomas, 1957).

Figure 6.9 illustrates the depth distributions of the $\mathrm{H}$-alpha photon production (RP) and destruction (RD) ratios, defined for the simplified case of a two level plus continuum model hydrogen atom with the Lyman lines in detailed balance (Chapter 5, eq. 5.2). For model 1, $\mathrm{RP}>1$ and $\mathrm{RD} \sim 1$ at the location corresponding to maximum chromospheric $\mathrm{Ne}(2.8 \mathrm{E} 11 \mathrm{~cm}-3$, at $\mathrm{Te}=6900 \mathrm{~K})$. 


\section{FIGURE 6.8}

The variation of $\log \left(\mathrm{S}_{\mathrm{H} \alpha}\right)(-)$ and $\log \left(\mathrm{B}_{\nu}\right)$

$(\ldots$.$) with respect to \log (M)$ is shown for models 1,2 and 3 . The mass column density points corresponding to $\mathrm{H} \alpha$ optical depth unity at $l$ ine centre and $\Delta \lambda=0.6 \AA$ are annotated by $(+)$ and (๑), respectively. Note the enhanced chromospheric $\mathrm{S}_{\mathrm{H} \alpha}$ in models 2 and 3 and, in particular, the increased $\mathrm{H} \alpha$ wing opacity at $\Delta \lambda=0.6 \AA$ in model 3 . 


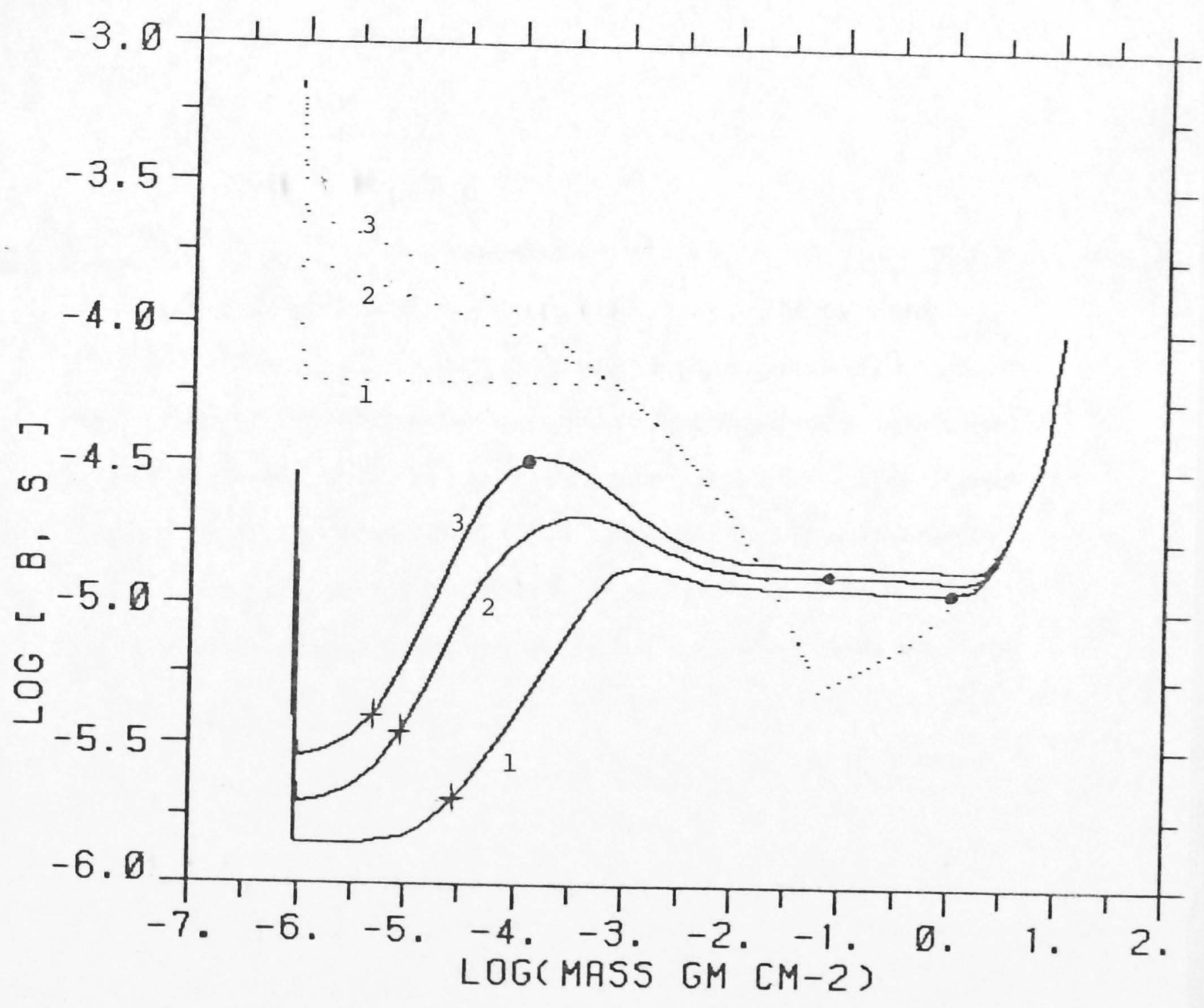




\section{FIGURE 6.9}

The variation of $\log (\mathrm{RP})(-)$ and $\log (\mathrm{RD})(\ldots)$ with respect to $\log (M)$ is shown for models 1,2 and 3 . RP measures the importance of $\mathrm{H} \alpha$ photon production by direct electron collisional excitations $(2 \rightarrow 3)$, relative to indirect photoionisations $(2 \rightarrow$ continuum $\rightarrow 3)$. RD gauges the importance of $\mathrm{H} \alpha$ photon destruction by collisional deexcitations $(3 \rightarrow 2)$, relative to photoionisations $(3 \rightarrow$ continuum $\rightarrow 2$ ). Expressions for these ratios are given in Chapter Five (eq. 5.2). 


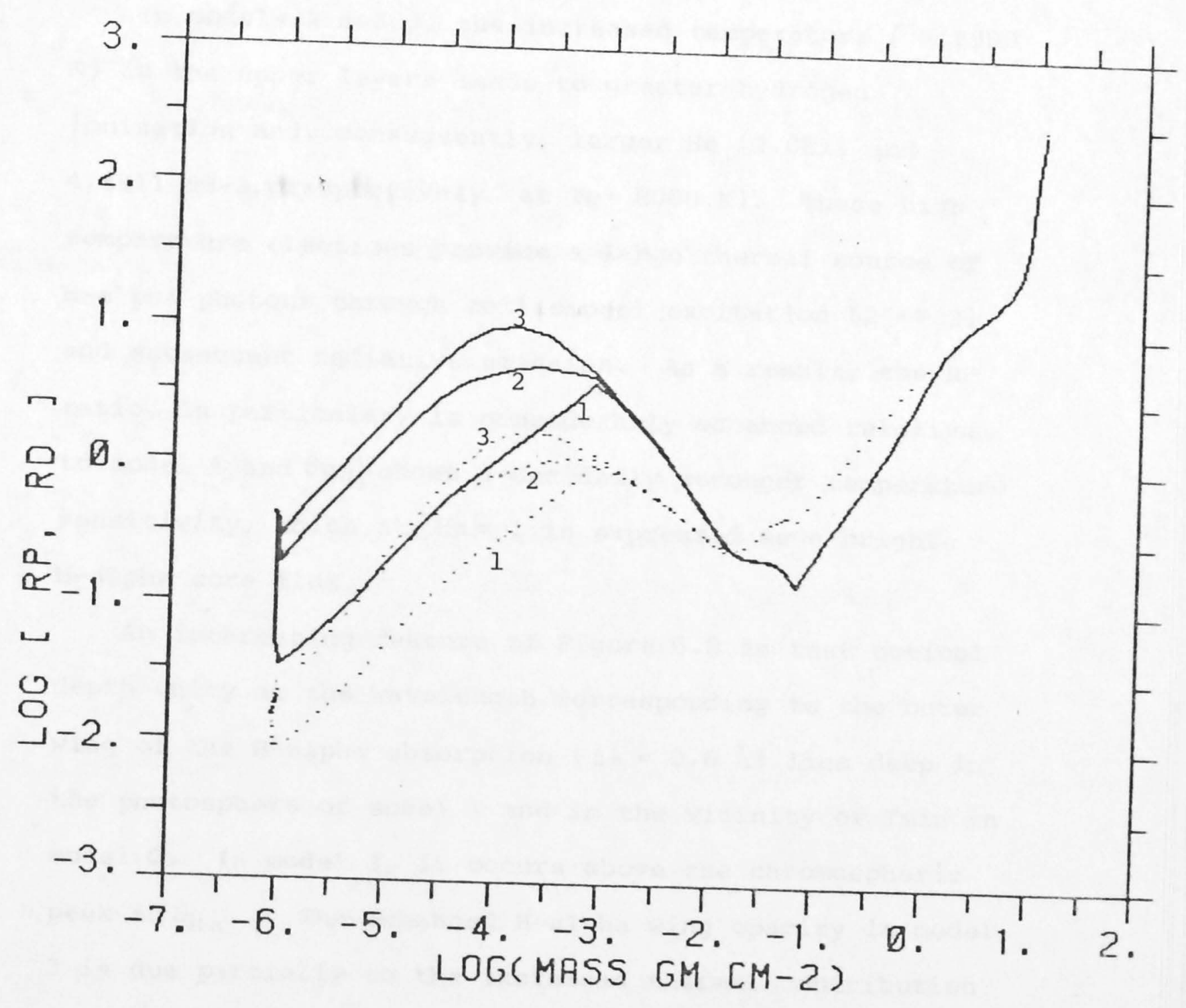


Thus, direct electron collisional excitation processes are particularly dominant relative to indirect radiative in influencing the H-alpha statistical equilibrium. This explains partially the weak sensitivity of the H-alpha line emitted by this model to the variations in background radiation temperatures described previously (see Figure 6.4).

In models 2 and 3 , the increased temperature ( > 6900 K) in the upper layers leads to greater hydrogen ionisation and, consequently, larger $\mathrm{Ne}(2.0 \mathrm{Ell}$ and 4.8Ell $\mathrm{cm}-3$, respectively, at $\mathrm{Te}=8000 \mathrm{~K})$. These high temperature electrons provide a large thermal source of H-alpha photons through collisional excitation $(2 \rightarrow 3)$ and subsequent radiative emission. As a result, the RP ratio, in particular, is considerably enhanced relative to model 1 and $\mathrm{S}_{\mathrm{H} \alpha} \mid$ shows a decidedly stronger temperature sensitivity, which at ${ }^{\tau} \mathrm{H} \alpha=1$ is expressed as a bright H-alpha core flux.

An interesting feature of Figure 6.8 is that optical depth unity at the wavelength corresponding to the outer wing of the H-alpha absorption $(\Delta \lambda=0.6 \AA)$ lies deep in the photosphere of model $l$ and in the vicinity of Tmin in model 2. In model 3 , it occurs above the chromospheric peak in $\mathrm{S}_{\mathrm{H} \alpha}$. The enhanced $\mathrm{H}$-alpha wing opacity in model 3 is due partially to the increased thermal contribution to the H-alpha Doppler width, $\Delta \lambda_{D}$, caused by the steep temperature gradient above the hinge point. The attendant increase in $\Delta \lambda_{D}$ in these upper layers is responsible for inhibiting the escape of photons in the 
lower layers (Athay, 1972). This effect, in conjunction with the high electron density, acts to enhance further $\mathrm{S}_{\mathrm{H} \alpha}$ relative to models 1 and 2 . Incipient emission wings develop as a consequence of the greater contribution to the wing flux by photons emanating from the optically thick chromospheric layers of this model. Similar arguments apply to the formation of the H-alpha emission components in the high pressure distribution.

\section{$\underline{6.2 .3 \text { Discussion }}$}

In principle, enhanced upper temperature models such as 2 and 3, when combined with appropriate degrees of macroturbulence, can lead to an optimum fitting $\mathrm{H}-\mathrm{al}$ pha profile for $70 \mathrm{Oph}$ A. An important constraint on the validity of such configurations concerns their consistency with $\mathrm{Ca}$ II and Mg II observations. For example, the Ca II K2 emission produced by these models is brighter than for the reference case (see Figure 6.10). Examination of the Ca II $K$ optical depth scales for the latter model reveals that unit optical depth at the wavelength corresponding to the $\mathrm{K} 2$ feature occurs at the base of the plateau region. Furthermore, this point coincides with the peak in the Ca II $K$ frequency independent source function. Thus, the Ca II K flux will be sensitive particularly to the thermal structure in this region. Increasing the temperature above $\log (M)=$ -3.1 will, by virtue of enhanced collisional coupling with $B(T)$ and increased $C a$ II $K$ optical depth, act to strengthen the $\mathrm{K} 2$ emission. 
FIGURE 6.10

CRD $\mathrm{Ca}$ II $\mathrm{K}$ profiles are shown for models 1 ( - ), $2(\ldots)$ and $3(x \times x x)$. As expected, the Ca II K2 feature brightens with increasing temperature gradient in the upper chromosphere. The fluxes at $\mathrm{Kl}$ are unchanged between the models. 


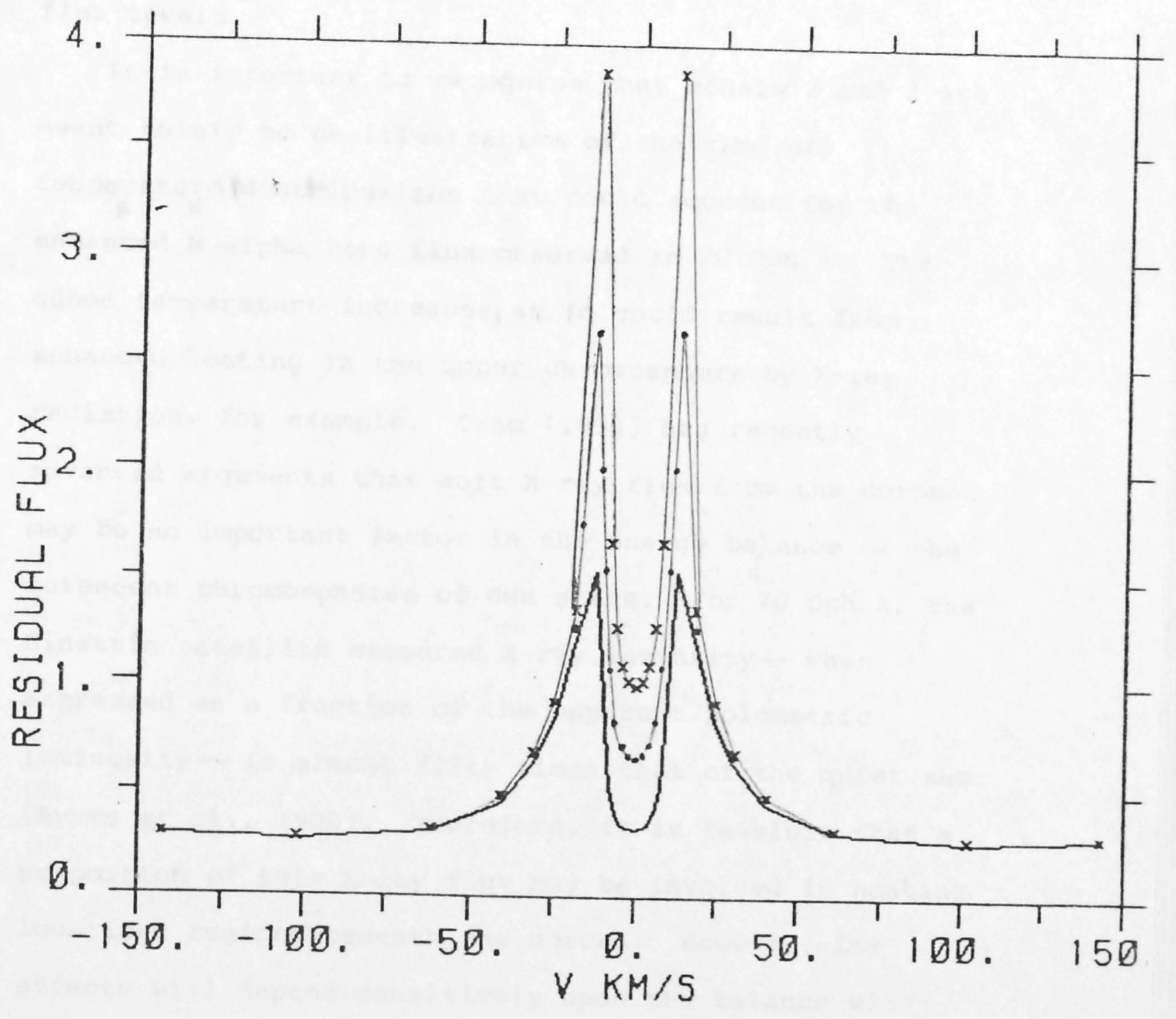


Similar arguments apply to the optically thicker Mg II $h$ and $k$ lines which thermalise in the higher chromospheric layers, and would accordingly undergo an emission strengthening with increasing upper chromospheric temperature gradient (Kelch, 1978). Consequently, we anticipate that these models would tend to overpredict observed $70 \mathrm{Oph} \mathrm{A} \mathrm{Ca} \mathrm{II} \mathrm{and} \mathrm{Mg} \mathrm{II} \mathrm{emission}$ flux levels.

It is important to recognise that models 2 and 3 are meant solely to be illustrative of the kind of temperature distributions that could account for the enhanced H-alpha core flux observed in $70 \mathrm{Oph} \mathrm{A.} \mathrm{The}$ adhoc temperature increases at To could result from enhanced heating in the upper chromosphere by $x$-ray radiation, for example. Cram (1982) has recently advanced arguments that soft $\mathrm{X}$-ray flux from the corona may be an important factor in the energy balance of the quiescent chromospheres of dMe stars. For $70 \mathrm{Oph} A$, the Einstein satellite measured $\mathrm{X}$-ray intensity - when expressed as a fraction of the apparent bolometric luminosity - is almost fifty times that of the quiet sun (Ayres et al., 1982). Therefore, it is feasible that a proportion of this $\mathrm{X}$-ray flux may be involved in heating localised regions beneath the corona. However, its effects will depend sensitively upon the balance with competing heating agents (e.g. thermal conduction or magneto-acoustic phenomena) and the efficiency of plasma cooling (e.g. radiative losses in effectively thin resonance lines). 
Our calculations do not exclude the possibility that a more improved fit to the H-alpha profile can be achieved by different chromospheric configurations. For example, models with slightly higher Tz pressures. However, we do discriminate against excessively high $\mathrm{TZ}$ pressures ( $\log M o>-4.0$ ) on the grounds that,

(1) models such as 4 would produce optically thick H-alpha emission lines with discrepantly large central intensities; and

(2) according to the theoretical calculations of Kelch (1978), high pressure modeis with $\log ($ Mo) $>-4.25$ (see, for example, his model $\mathrm{C}$ ) would lead inevitably to Mg II $h$ and $k$ emission flux levels above acceptable limits of observation.

The results of our preliminary calculations suggest that it is difficult to derive a single component chromosphere model for $70 \mathrm{Oph}$ A that is consistent simultaneously in the H-alpha, Ca II and Mg II lines. The important point remains that the chromospheric structure of model 1, although alledgedly consistent with $\mathrm{Ca}$ II and Mg II emission in $70 \mathrm{Oph} \mathrm{A}$, does not reproduce H-alpha compatible with observation. Thus, we surmise that homogeneity may not be a valid assumption for the chromosphere of this active dwarf. In particular, the $\mathrm{H}$-alpha core and $\mathrm{Ca}$ II $\mathrm{K}$ emission may be more likely formed in different laterally spaced chromospheric regions of varying electron temperature. Evidence in 
support of this heterogenous scenario for 70 Oph A is summarised in section 6.4 .

\subsection{ARCTURUS CHROMOSPHERE MODELS}

\subsubsection{Description of Models}

In this section we consider the H-alpha profile of Arcturus. Theoretical profiles are synthesised from chromosphere models of differing temperature gradient, $\mathrm{dT} / \mathrm{d} \log (\mathrm{M})$, and are compared with observation. Defining characteristics for the three models treated in this investigation are compiled in Table 6.2, while the corresponding $\mathrm{Te}-\log (\mathrm{M})$ distributions are plotted in Figure 6.11.

The photospheric region in each model is represented by a flux constant, line blanketed model atmosphere adapted from Gustafsson et al. (1975), with $\operatorname{Teff} / \log (\mathrm{g}) /[\mathrm{Fe} / \mathrm{H}]=4250 / 1.7 /-0.5$ and constant $2 \mathrm{~km} / \mathrm{s}$ microturbulence. Chromospheric segments are characterised by linear temperature rises in $\log (M)$ between $[\mathrm{Tmin}, \log \mathrm{Mmin}]=[3200 \mathrm{~K}, 0.25]$ and $\mathrm{TO}=8000 \mathrm{~K}$ at $\log ($ Mo $)=-5.5,-5.0$ and -4.4 for models $A, B$ and $C$, respectively. The latter choices of Mo bracket the range of "top masses" that is consistent with observed Ca II and Mg II emission in Arcturus (Ayres and Linsky, 1975b). Above Mo, the $\mathrm{Tz}$ in each model is simulated by a sharp temperature increase to $20000 \mathrm{~K}$ over a $0.6 \mathrm{dex}$ range in $\log (M)$. Additionally, a microturbulent velocity field - which increases linearly in Log(M) - from $2 \mathrm{~km} / \mathrm{s}$ 
FIGURE 6.11

Te-Log(M) distributions for the three Arcturus

chromosphere models considered in this investigation. 


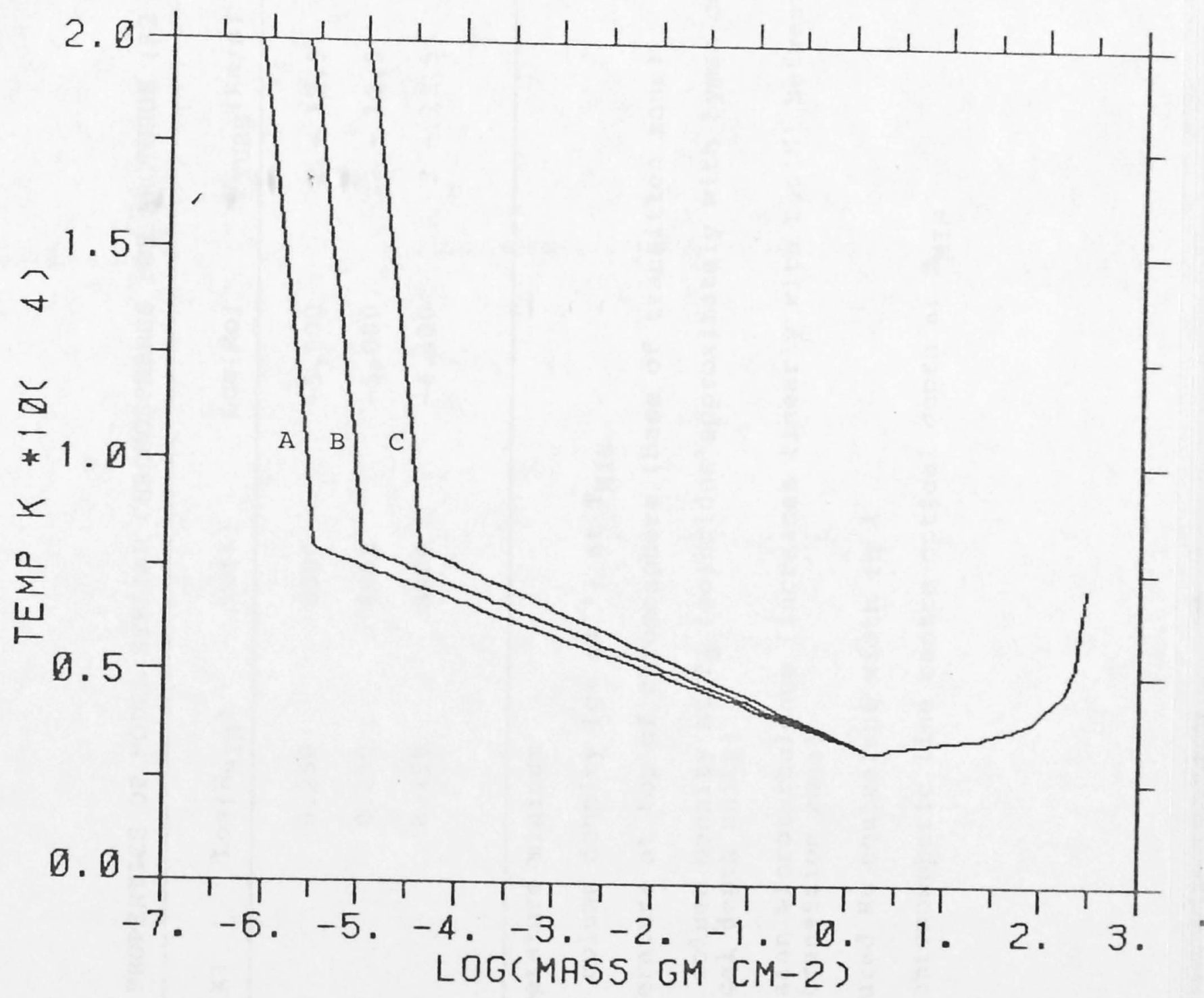


TABLE 6.2

PROPERTIES OF MODEL STELLAR CHROMOSPHERES FOR ARCTURUS (K2 III)

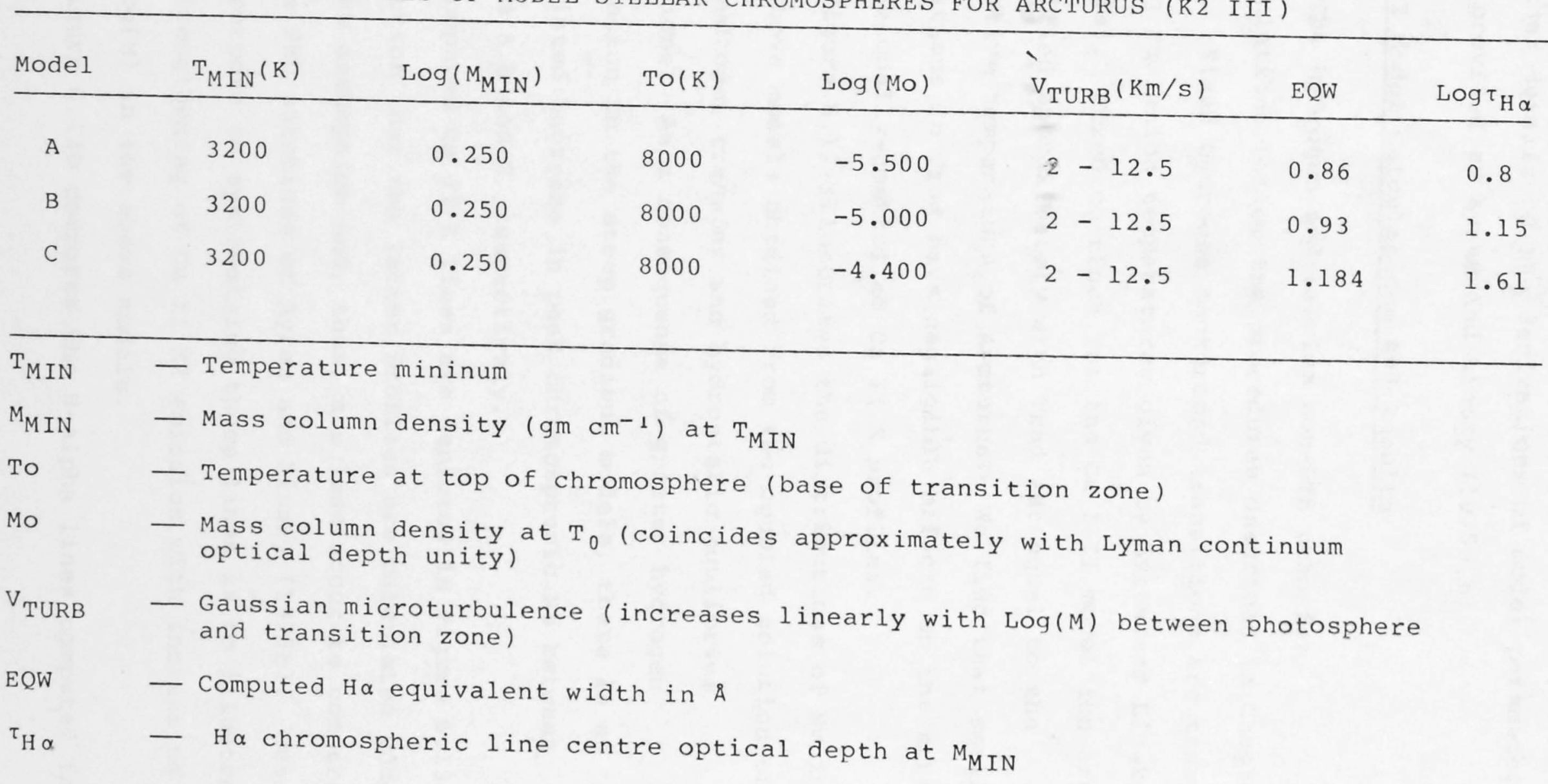


at Mmin to $12.5 \mathrm{~km} / \mathrm{s}$ at Mo is included in each model.

Further details of the derivations of model parameters are provided by Ayres and Linsky $(1975 \mathrm{a}, \mathrm{b})$.

\subsubsection{Model Calculations And Results}

The hydrogen and calcium non-LTE transfer calculations follow the procedures described in Chapter Four. Fixed hydrogen background transitions are treated using radiation temperatures given by Ayres and Linsky (1975a). Fixed continua for the Ca I-II model ion are specified approximately with Trad set equal to the effective temperature of Arcturus. We find that small variations in Trad have negligible effects on the strong collisionally-controlled Ca II K profiles.

Figure 6.12 illustrates the distributions of $\mathrm{Ne}$ in the above models obtained from the coupled solution of the hydrogen transfer and hydrostatic equlibrium equations. As a consequence of greater hydrogen ionisation in the steep gradient models, there is an associated increase in peak chromospheric Ne between models $A, B$ and $C$, respectively.

Computed Ca II K lines are depicted in Figure 6.13a. We caution that the latter profiles are calculated under the CRD assumption and, thus, are less accurate compared to the PRD solutions of Ayres and Linsky (1975b). Our only purpose in synthesising these lines is to illustrate the strengthening of $\mathrm{Ca}$ II K2 emission with increasing $\mathrm{dT} / \mathrm{dLog}(\mathrm{M})$ in the above models.

Figure $6.13 \mathrm{~b}$ compares the $\mathrm{H}-\mathrm{al}$ pha lines computed from 


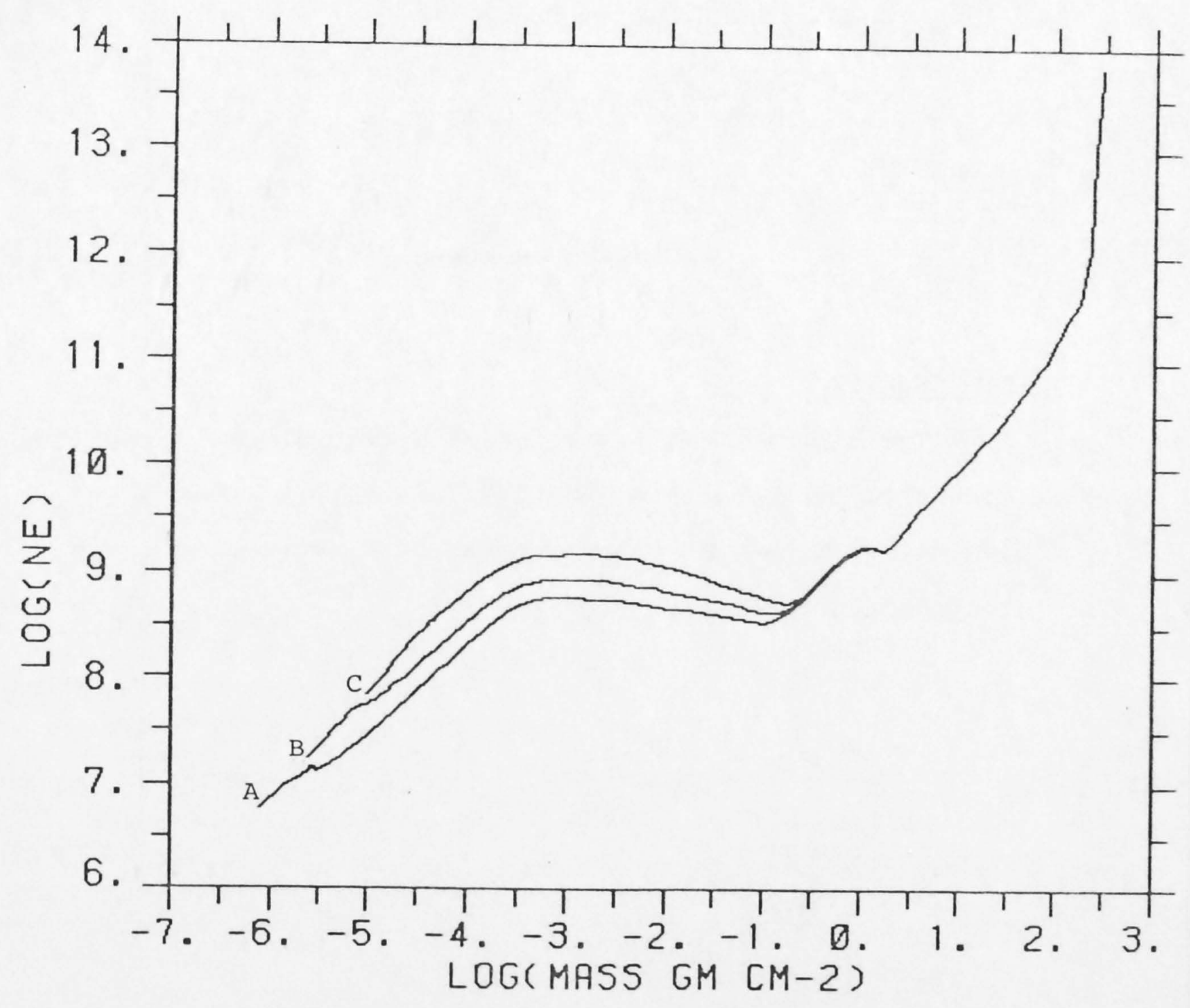


FIGURE 6.12

The distribution of $\log (\mathrm{Ne})$ with respect to $\log (M)$ for models $A, B$ and $C$. Note the almost two orders of magnitude reduction in peak chromospheric Ne between model $\mathrm{C}$ and 70 Oph A model 1 (cf. Fig. 6.2). 


\section{FIGURE $6.13 \mathrm{a}$}

The CRD Ca II K profiles computed from models A (...), $B(x \times x x)$ and $C(-)$ are compared to illustrate the emission strengthening with increasing chromospheric temperature gradient. 


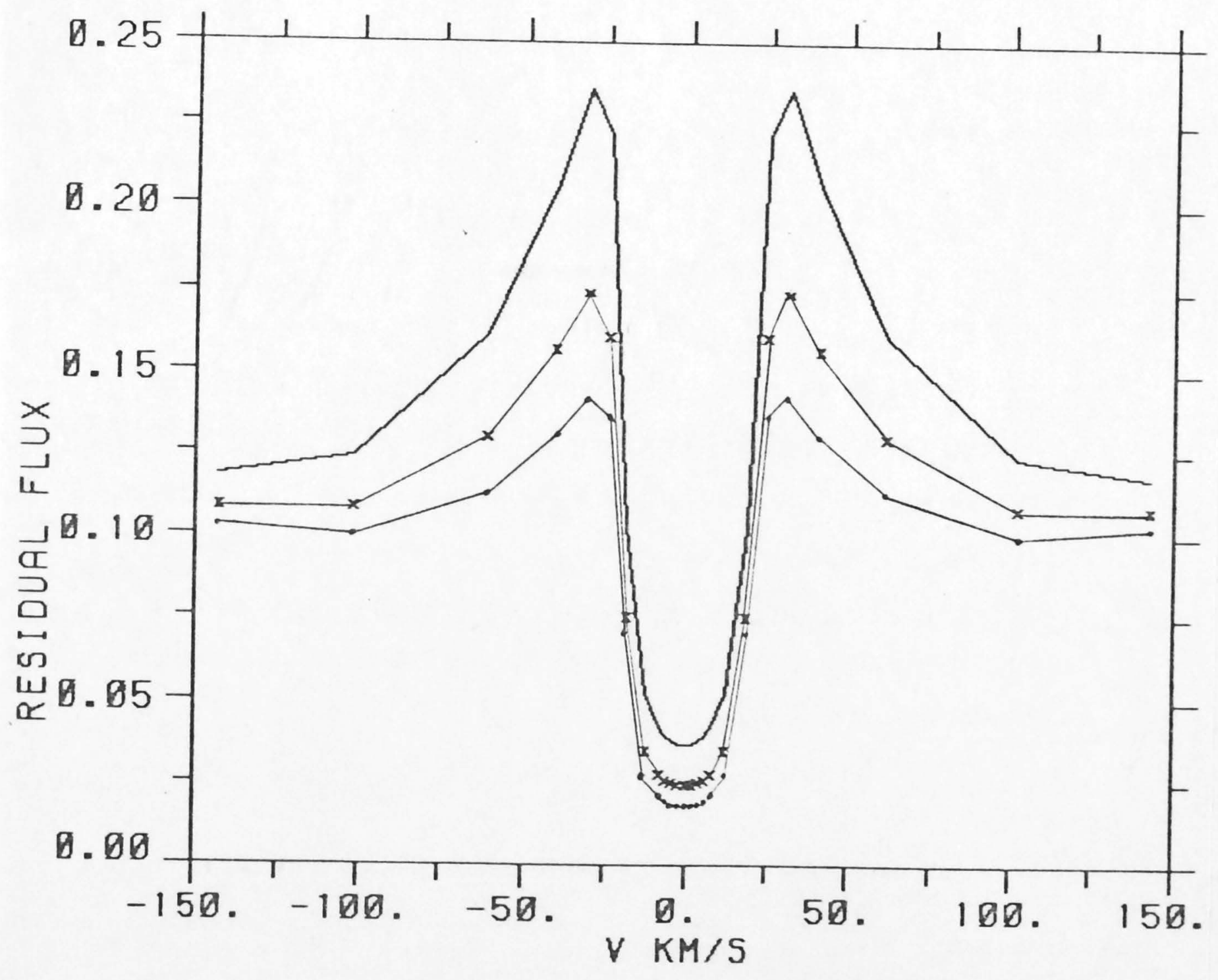




\section{FIGURE $6.13 \mathrm{~b}$}

Computed $\mathrm{H} \alpha$ lines from models A $(\ldots), \mathrm{B}(\mathrm{x} \times \mathrm{x} x)$ and

C (-) are compared with the observed Arcturus $\mathrm{H} \alpha$ profile (on common velocity and continuum flux scales). A favorable fit to the observed line width is provided by the model $C$ profile. Note also the effect of decreasing Rc with increasing chromospheric temperature gradient in models A, B and $\mathrm{C}$, respectively. 


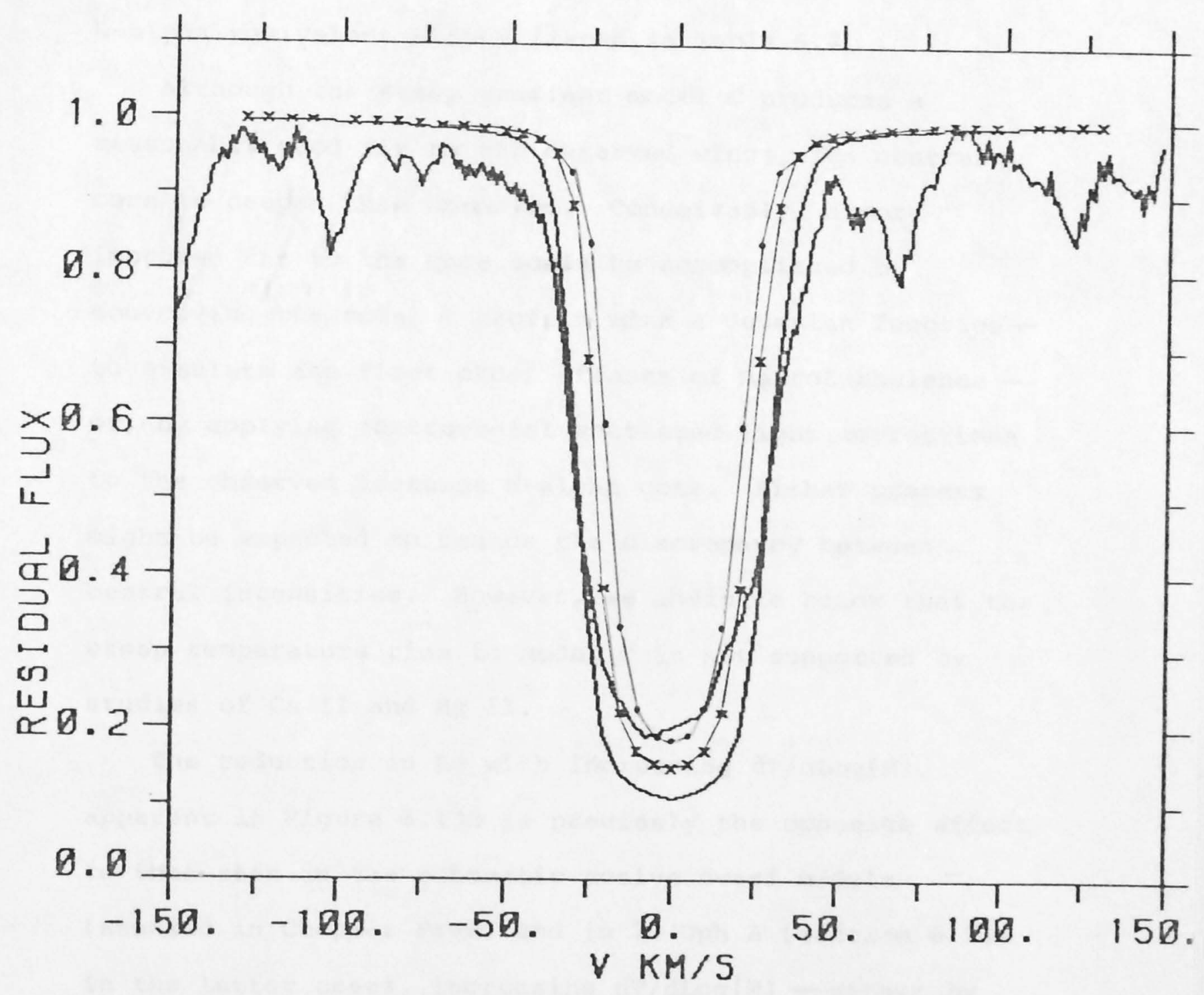


the three chromosphere models with our observed Arcturus profile (Chapter Two, Figure 2.2a). A similar result to that demonstrated previously by Eriksson (1980) is found here; namely, for a fixed chromospheric gradient in Vturb, the H-alpha profile tends to become deeper (decreasing $\mathrm{RC}$ ) and broader with increasing $\mathrm{dT} / \mathrm{dLog}(\mathrm{M})$. This enhancement in line strength is reflected in the H-alpha equivalent widths listed in Table 6.2.

Although the steep gradient model $C$ produces a reasonably good fit to the observed wings, its central core is deeper than observed. Conceivably, a more improved fit to the core could be accomplished by convolving the model $C$ profile with a Gaussian function to simulate the first order effects of macroturbulence or, by applying instrumental scattered light corrections to the observed Arcturus H-alpha core. Either process might be expected to reduce the discrepancy between central intensities. However, we indicate below that the steep temperature rise in model $\mathrm{C}$ is not supported by studies of $\mathrm{Ca}$ II and $\mathrm{Mg}$ II.

The reduction in $\mathrm{RC}$ with increasing $\mathrm{dT} / \mathrm{dLOg}(\mathrm{M})$ apparent in Figure $6.13 \mathrm{~b}$ is precisely the opposite effect to that seen in the schematic active dwarf models (studied in Chapter Five) and in 70 Oph A (section 6.2). In the latter cases, increasing $\mathrm{dT} / \mathrm{dLog}(\mathrm{M})$ - either by increasing Mo or To-resulted in significant $\mathrm{H}-\mathrm{al}$ pha core intensity enhancements. We discuss these two contrasting behaviours in the following section. 


\subsection{Discussion}

Eriksson (1980) has indicated that the chromospheric gradient in Vturb is a critical parameter affecting the $\mathrm{H}$-alpha (and H-gamma) line strength of Arcturus. In particular, he finds that while high pressure models (Log Mo $\sim-4.0)$ can provide acceptable fits to the observed H-alpha strength, equally good agreement can be achieved with low pressure models ( Log Mo $\sim-5.0$ ) combined with steep chromospheric rises in Vturb. Since large values of Mo ( $\log$ Mo > -4.5) are not supported by Ca II and Mg II observations (Ayres and Linsky, 1975b) - nor by studies of H-epsilon emission in Arcturus (Ayres and Linsky, 1975a) - Eriksson (1980) concludes that the observed strengths of the hydrogen lines necessitate the existence of a steep chromospheric Vturb gradient.

Permissible microturbulence models are tightly constrained by the requirement of fitting the $\mathrm{K} 2$ emission peaks. The $2-12.5 \mathrm{~km} / \mathrm{s}$ Vturb variation adopted in this investigation is actually an upper limit to the chromospheric Vturb gradient that is consistent with the Ca II H, K and infra-red triplet lines of Arcturus (Ayres and Linsky, 1975b). Given this Vturb variation, the results of the $\mathrm{H}-\mathrm{alph}$ computations indicate that the range of semi-empirical Arcturus chromosphere temperature models $(-5.0<\log$ Mo $<-4.5)$ - inferred from studies of $\mathrm{Ca}$ II and Mg II- is consistent in predicting H-alpha profiles to within acceptable limits of observational uncertainty. 
Finally, we comment on the behaviour of the Arcturus $\mathrm{H}-\mathrm{alpha}$ line core with increasing $\mathrm{dT} / \mathrm{d} \log (\mathrm{M})$. Figure 6.14 compares the distributions of $\mathrm{S}_{\mathrm{H} \alpha}$ and $\mathrm{B}(\mathrm{T})$ in models $\mathrm{A}$ and $\mathrm{C}$. As a consequence of its photoelectric nature - due to relatively low chromospheric $\mathrm{Ne}-\mathrm{S}_{\mathrm{H} \alpha}$ in both models is uncoupled from the chromospheric temperature rise. Consequently, the H-alpha core flux in Arcturus is relatively insensitive to upper chromospheric thermal structure. Therefore, the decrease in Rc (or, increase in equivalent width) with increasing $\mathrm{dT} / \mathrm{dLog}(\mathrm{M})$ displayed in Figure $6.13 \mathrm{~b}$ is primarily in response to the enhanced H-alpha chromospheric optical depth (see Table 6.2), resulting from the larger $\mathrm{Hn}=2$ column density in the hotter steeper gradient models.

Thus, in contrast to the H-alpha profiles of the active dwarf stars alluded to previously, we would not expect a direct dependence between H-alpha core flux and $\mathrm{dT} / \mathrm{dLOg}(\mathrm{M})$ in low gravity stars such as Arcturus. Correspondingly, an absence of explicit correlation between RC and Ca II K emission strength is implicated for giants (and supergiants). Indeed, this dichotomous behaviour between dwarfs and giants is reflected in the Rc - Ik diagrams illustrated in Chapter Three (Figures $3.3 \mathrm{a}, \mathrm{b}$ and $\mathrm{c})$. The lack of correlation, and high dispersion, between Rc and Ik amongst giants and supergiants is more likely a consequence of enhanced chromospheric velocity fields - random and systematic affecting the $\mathrm{H}-\mathrm{alpha}$ and $\mathrm{Ca}$ II $\mathrm{K}$ cores in these low gravity stars (cf. Chapter Three, section 3.5). 
FIGURE 6.14

$\log \left(\mathrm{S}_{\mathrm{H} \alpha}\right)(\longrightarrow)$ and $\log \left(\mathrm{B}_{\nu}\right)(\ldots)$ are plotted

with respect to $\log (M)$ for models $A$ and $C . S_{H \alpha}$ in both models is uncoupled from $B_{\nu}$ in the chromosphere. 


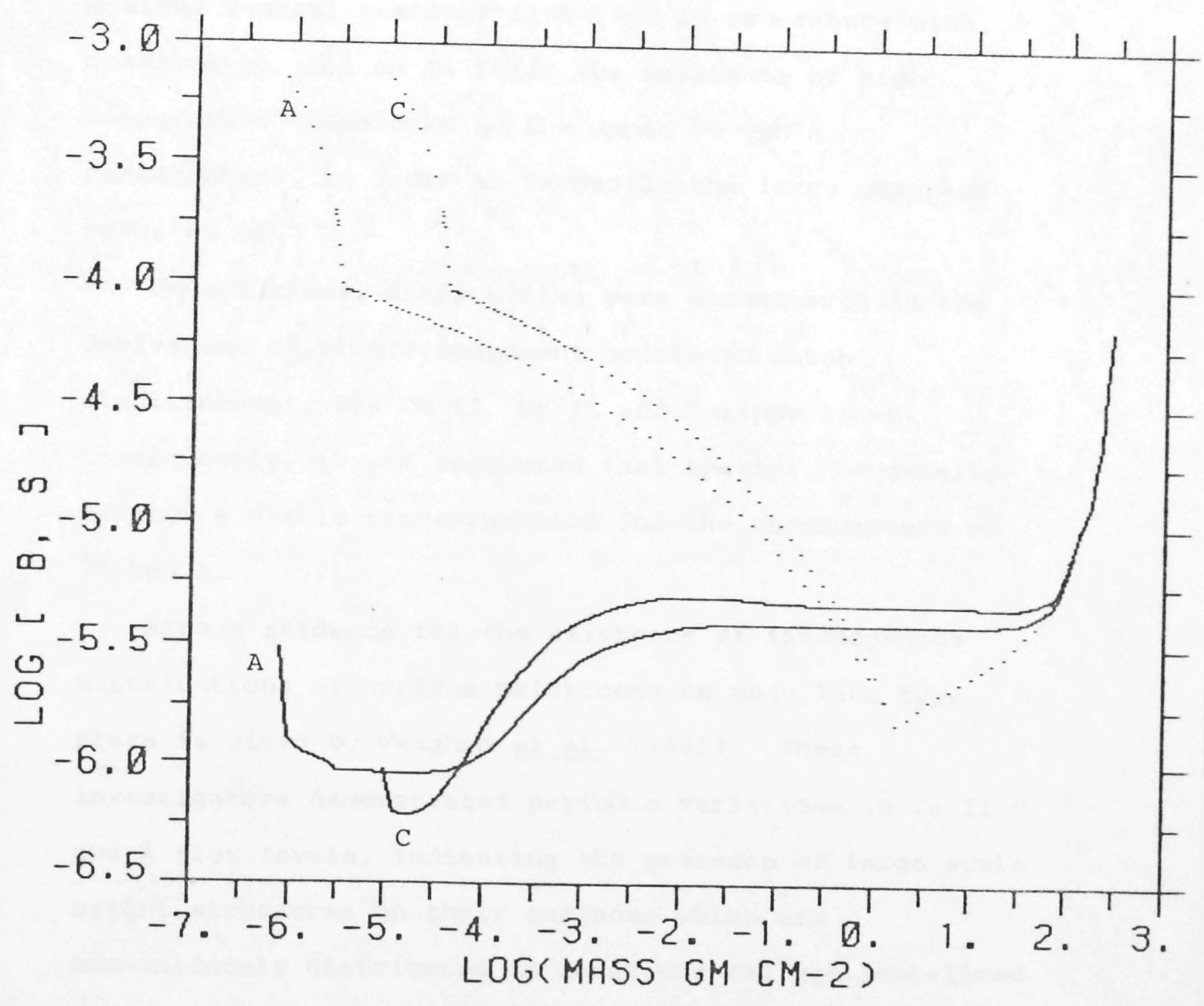




\section{$\underline{6.4 \text { CONCLUSION }}$}

We commenced this investigation by examining the degree to which the $70 \mathrm{Oph}$ A chromosphere model derived by Kelch (1978) was self-consistent in its reproduction of the observed $\mathrm{H}-\mathrm{al}$ pha profile for this active late type dwarf. The apparent failure of the model to predict the $\mathrm{H}-\mathrm{alpha}$ central residual flux (RC) in accordance with observation, led us to infer the existence of high temperature components in the upper $70 \mathrm{Oph} \mathrm{A}$ chromosphere, in order to reconcile the large observed value of Rc.

Nevertheless, difficulties were encountered in the derivation of single component models to match simultaneously the $\mathrm{Ca}$ II, Mg II and $\mathrm{H}-\mathrm{al}$ pha lines. Consequently, it was suggested that spatial homogeneity was not a viable representation for the chromosphere of 70 Oph A.

Strong evidence for the existence of inhomogenous distributions of surface brightness on many late type stars is given by Vaughan et al. (1981). These investigators demonstrated periodic variations in $\mathrm{Ca}$ II $\mathrm{H}$ and $K$ flux levels, indicating the presence of large scale bright structures on their surfaces which are non-uniformly distributed in longitude, and are long-lived compared to a rotational period. Extensive studies (Wilson, 1978) of long term cyclical variations of $\mathrm{H}$ and $\mathrm{K}$ in 91 main sequence stars - including 70 Oph $\mathrm{A}$ - have provided evidence for short term small amplitude 
fluctuations, which may be attributed to localised regions of "patchiness" on their surfaces.

The notion of heterogeneity for the $70 \mathrm{Oph} A$ chromosphere is reinforced further by the detection of magnetic fields of order 2 kilogauss, covering perhaps 10 \% of its surface (Robinson et al., 1980). Therefore, it is likely that such fields, in addition to augmenting chromospheric non-radiative heating (Stein, 1981), will play an important role in chanelling the flow of mass and energy in diverging flux tube configurations such as have been proposed for the sun (Giovanelli, 1980).

On the basis of mounting evidence for the existence of heterogenous atmospheric regions on many late type stars, and in view of the preliminary results of our H-alpha calculations, we conclude that a more viable model for $70 \mathrm{Oph} \mathrm{A}$ would consist of multicomponent structures, with the H-alpha core profile being a sensitive indicator of hot, upper chromospheric components.

Finally, for the case of Arcturus, we find that chromospheric temperature and microturbulence models, inferred from the Ca II and Mg II lines, give reasonable fits to observed H-alpha. Thus, we conclude that insofar as obtaining simultaneous agreement between theory and observation of the latter spectral features, the single component models considered in section 6.3 provide adequate representations for the chromosphere of Arcturus . 


\section{CHAPTER SEVEN}

\section{THESIS SUMMARY AND FUTURE WORK}

The aim of this thesis has been to illustrate - from both an empirical and theoretical standpoint - the usefulness of the H-alpha profile as a chromospheric diagnostic in late type stars. Specifically, we have focussed on comparisons between the H-alpha line and the well-established chromospheric indicator Ca II K, as a means of discerning features of common chromospheric origin. This concluding chapter summarises the principle results of this investigation, and outlines several suggestions for future work to complement and extend that described here.

The observational data base (Chapter Two) comprised an extensive and homogenous set of high resolution H-alpha and $\mathrm{Ca}$ II $\mathrm{K}$ echelle spectra, which covers a wide range of spectral type and luminosity class. A coordinated analysis of these two lines (Chapter Three) has yielded the following results:

(i) a weak correlation of increasing H-alpha absorption full width at half depth with increasing $\mathrm{Ca}$ II $\mathrm{K}$ Wilson-Bappu width or, equivalently, absolute visual luminosity. The Ho dependence upon wo is spectral type dependent and is somewhat scattered. Various factors 
contributing to this scatter include, the influence of photospheric damping wings in the H-alpha cores of dwarfs and macrovelocity induced $\mathrm{H}$-alpha asymmetries (and possible emission) in cool giants and supergiants. However, future corrections for these intrinsic stellar effects - for example, the fitting of Gaussian profiles to the H-alpha Doppler core in dwarfs (Lo Presto, 1971), and the symmetrising of $\mathrm{H}$-alpha in giants and supergiants using line bisector techniques (Imhoff, 1977) - may lead ultimately to an improved H-alpha width-luminosity correlation.

(ii) our repetition of the analysis of Fosbury (1973) has verified that the non-linearity in the empirical Ho-Wo relation can be understood partially, by taking into account the H-alpha chromospheric thermal broadening component; the latter provides a lower limit to Ho in low luminosity stars. An important corrollary of this treatment is that Ho-corrected for the thermal broadening contribution - can provide information on mean chromospheric non-thermal velocities, in particular microturbulence. For example, application of the Goldberg (1957) constant properties slab formula allows determinations of upper limits to chromospheric microturbulence from measurements of Ho. A comparison between these non-thermal velocites and the Wilson-Bappu width measurements has shown that, for many of the programme stars, the intensity point at which wo is measured is located beyond the nominal three Doppler 
widths limit separating the Doppler core from the damping wing of the line absorption profile. We deduce from this result that the Ayres (1979) hypothesis of damping controlled and, hence, opacity broadened wo is a plausible alternative to the Doppler interpretations documented extensively in early literature (cf. Chapter One, section 1.2.1).

The constant properties atmosphere approximation implicit in the slab model limits severely its practical application to empirical data. Realistic chromosphere models of numerous late type stars (refer to the "Stellar Model Chromospheres Series", by Linsky and collaborators) dramatically illustrate the depth-dependent nature of many important chromospheric quantities, in particular the line Doppler width parameter. Furthermore, line profile asymmetries evident in numerous late type giants and supergiants, provide clear indications for the existence of systematic macro-velocity fields; the latter have a profound influence on the radiative transfer properties of a stellar atmosphere, and subsequently the widths, shapes and strengths of spectral lines such as H-alpha (Mallik, 1982). Finally, the phenomenon of macroturbulence (random large scale atmospheric motions) has been shown to be important in affecting the widths of spectral lines in late type giants and supergiants (Smith and Dominy, 1979; and references therein).

In view of the above complications, our simplified approach to estimating the elusive Doppler parameter from H-alpha widths should only be construed as a first cut 
attempt at utilising the H-alpha profile as a diagnostic of chromospheric non-thermal velocities. Clearly, future and more accurate determinations of chromospheric microturbulence from H-alpha widths must entail an explicit account of height variations of chromospheric parameters, and the existence of dynamical motions on scales larger than a photon mean free path.

\section{(iii) comparisons between the shapes of H-alpha} absorption and $\mathrm{Ca}$ II $\mathrm{K}$ emission cores of late type giants and supergiants have revealed a correlation in asymmetry morphology. In particular, red-dominated Ca II $\mathrm{K}$ asymmetry - preponderant among low gravity stars cooler than the Stencel (1978) wind boundary - is reflected in redward asymmetry in the corresponding $\mathrm{H}$-alpha aborption core. This correlation highlights the usefulness of the H-alpha profile as a tracer for chromospheric velocity fields.

A more striking effect, seen among several of the $G$ and $\mathrm{K}$ supergiants, is an apparent one-to-one mapping between fine structures observed in the $\mathrm{K} 3$ component of Ca II K, and those evident in the asymmetric core of H-alpha. This correspondence parallels a similar correlation observed between $\mathrm{H}-\mathrm{al}$ pha and $\mathrm{Ca}$ II $\mathrm{K}$ features in the solar chromosphere (McMath et al., 1956). It suggests that dynamical processes controlling the behaviours of these two spectral features in the sun are also operating in other late type stars, hence reinforcing the concept of a solar-stellar connection. 
Many of the cool, luminous stars displayed in Chapter Two show evidence of enhanced blue-shifted Ca II K absorption features (K4), attributable to the effect of a circumstellar envelope. The contaminating influence of the K4 component severely inhibits the accurate use of the $\mathrm{Ca}$ II $\mathrm{K}$ line asymmetry as a diagnostic of intrinsic chromospheric velocity fields. On the other hand, the corresponding $\mathrm{H}-\mathrm{al}$ ha profiles of these stars do not appear to suffer from this defect; the bulk of H-alpha absorption is formed in the chromosphere with no circumstellar contribution (Mallik, 1982). This trait promotes the asymmetric H-alpha absorption profile as a non-ambiguous probe of chromospheric dynamical conditions.

Consequently, the use of H-alpha in conjunction with the $\mathrm{Ca}$ II $\mathrm{K}$ line (and also $\mathrm{Mg}$ II $\mathrm{h}$ and $\mathrm{k}$ ) in future numerical simulation studies of expanding chromospheres, will prove invaluable in constraining velocity models of late type stars.

(iv) finally, a study of the H-alpha profile in $G$ and $K$ dwarfs displaying a variety of $\mathrm{Ca}$ II $\mathrm{K}$ emission strengths - indicative of different degrees of chromospheric activity - has demonstrated that the central residual flux of H-alpha absorption increases with increasing $\mathrm{Ca}$ II $K$ brightness. This result suggests that the H-alpha core may provide a viable diagnostic of chromospheric activity, such as the $K$ emission has proved to be in past studies (cf. Kelch et al., 1979). 
The enhancement of H-alpha core intensity among the active dwarfs parallels the well-known contrast of chromospheric plages seen in H-alpha filtergrams of the solar surface (Athay, 1976). This is another manifestation of a solar-stellar connection. Preliminary non-LTE transfer calculations (Chapter Five) have indicated that the parameterisation of enhanced chromospheric activity by steep chromospheric temperature gradients - as invoked in past solar plage studies (Shine and Linsky, 1974) - is consistent with the $\mathrm{H}$-alpha core brightening observed among active main-sequence stars. In particular, it was demonstrated that electron collisional coupling between the H-alpha source function and the Planck function offered a plausible explanation for this core brightening phenomenon. Furthermore, the latter indicates that the $\mathrm{H}$-alpha core in active dwarfs originates in regions of higher temperature and density than the quiet sun chromosphere precisely the important attribute of magnetic active regions revealed in the $\mathrm{Ca}$ II $\mathrm{K}$ modelling of plages.

Consequently, future extensions of the previous plage studies to investigations of $\mathrm{H}$-alpha in a broad range of quiet and active dwarfs may elucidate the nature of magnetic active regions, and possibly may provide a better understanding of their important heating mechanisms (Athay, 1976).

Although the use of steep chromospheric temperature gradients is a reasonable approach to model active 
chromosphere stars, it is important to recognise that other processes can be equally effective in "filling-in" the H-alpha line core (e.g. rotation, or random turbulent motions on varying length scales; Shine, 1975). Hence, future studies of $\mathrm{H}$-alpha in active dwarfs which incorporate the effects of upward propagating acoustic and gravity waves - which have been investigated extensively for solar Ca II K (Heasley, 1975; Cram, 1978) - may possibly provide further important insights into the H-alpha-Ca II K contrasts alluded to previously. The study of the H-alpha profile in active late type dwarfs has clearly demonstrated the importance of this line as a diagnostic of thermodynamic conditions in active chromosphere regions. Moreover, it has verified the usefulness of the H-alpha profile as a constraining observational parameter in the determination of stellar model chromospheres. The latter aspect was addressed further in an investigation of the chromosphere models of 70 Oph A (KO V) and Arcturus (K2 III) (Chapter Six).

For Arcturus, it was found that the range of chromospheric models, consistent with Ca II and Mg II observations (Ayres and Linsky, 1975b), also produced $\mathrm{H}$-alpha profiles in reasonable agreement with observation. Thus, insofar as attaining simultaneous agreement between Ca II, Mg II and H-alpha lines, it appears that a single component chromospheric representation for Arcturus may be valid. In the case of the active dwarf 70 Oph $A$, it was found that the H-alpha profile calculated from a 
chromospheric model inferred from $\mathrm{Ca}$ II and Mg II studies (Kelch, 1978), was inconsistent with observation. In particular, the predicted $\mathrm{H}$-alpha central intensity was considerably deeper than observed. This discrepancy led us to infer the existence of high temperature components in the upper $70 \mathrm{Oph} A$ chromosphere, in order to reconcile the enhanced observed H-alpha central intensity. However, problems were encountered in attempting to obtain self-consistency between proposed temperature models/observations of Ca II K, Mg II $\mathrm{k}$ and $\mathrm{H}-\mathrm{al}$ pha. In view of these difficulties, we concluded that spatial homogeneity may not be a valid assumption for the chromosphere of $70 \mathrm{Oph} \mathrm{A}$, and that more viable chromosphere models for this star should, perhaps, comprise multi-component structures. It is hoped that these initial indications of chromospheric heterogeneity afforded by H-alpha observations will stimulate the future use of this line in multi-dimensional atmosphere studies of other non-solar stars (such as the work typified by Stenholm and Stenflo, 1977 and Owocki and Auer, 1980). 


\section{REFERENCES}

Abramowitz, M., and Stegun, I. 1968, Handbook of Mathematical Functions (N.B.S. Applied Mathematics Series \# 55).

Athay, R.G. 1972, Radiation Transport in Spectral Lines (Dordrecht : Reidel).

Athay, R.G. 1976, The Solar Chromosphere and Corona : Quiet Sun (Boston : Reidel).

Athay, R.G. 1981, Ap. J., 250, 709.

Athay, R.G., and Skumanich, A. 1968, Solar Phys., 3, 181.

Auer, L.H. 1967, Ap. J. (Letters), 150, L53.

Auer, L.H. 1973, Ap. J., 180, 469.

Auer, L.H., Heasley, J.N., and Milkey, R.W. 1972, Kitt Peak National Observatory Contribution No. 555 .

Auer, L.H., and Heasley, J.N. 1976, Ap. J. 205, 165.

Auer, L.H., and Mihalas, D. 1969, Ap. J. 158, 641 .

Auer, L.H., and Mihalas, D. 1970, M.N.R.A.S., 149, 65 .

Avrett, E.H. 1973, in "Stellar Chromospheres", eds. S.D. Jordan and E.H. Avrett (NASA SP-317), p27.

Ayres, T.R. 1975, Ap. J., 201, 799.

Ayres, T.R. 1977, Ap. J., 213, 296.

Ayres, T.R. 1979, Ap. J., 228, 509.

Ayres, T.R., and Linsky, J.L. 1975a, Ap. J., 201, 212.

Ayres, T.R., and Linsky, J.L. 1975b, Ap. J. 200, 660 .

Ayres, T.R., Linsky, J.L., Rodgers, A.W. and Kurucz, R.L. 1976, Ap. J., 210, 199.

Ayres, T.R., Linsky, J.L., and Shine, R.A. 1975, Ap. J. (Letters), 195, Ll2l.

Ayres, T.R., Linsky, J.L., Vaiana, G.S., Golub, L., and Rosner, R. 1982, preprint.

Bahcall, J.N. and Wolf, R.A. 1968, Ap. J., 152, 701.

Basri, G.S. 1979, PhD thesis, Univ. Colorado, Boulder Colorado.

Basri, G.S. 1980, Ap. J., 242, 1133. 
Basri, G.S., Linsky, J.L., Bartoe, J.D.F., Brueckner, G., and Van Hoosier, M.E. 1979, Ap. J., 230, 924.

Boesgaard, A.M., and Hagen, W. 1979, Ap. J., 231, 128.

Bonsack, W.K., and Culver, D. 1966, Ap. J., 145, 767.

Breene, R.G., 1957, Rev. Mod. Phys., 29, 92.

Cacciari, C., and Freeman, K.C. 1981, in "Physical Processes in Red Giants", eds. I. Iben, Jr. and A. Renzini, (Dordrecht : Reidel), p3ll.

Chiu, H.Y., Adams, P.J., Linsky, J.L., Basri, G.S., Maran, S.P., and Hobbs, R.W. 1977, Ap. J., 211, 453.

Cohen, J. 1976, Ap. J. (Letters), 203, L127.

Cram, L.E. 1976, Astr. Ap., 50, 263.

Cram, L.E. 1978, Astr. Ap., 70, 345.

Cram, L.E. 1982, Ap. J., 253, 768 .

Cram, L.E., Brown, D.R. and, Beckers, J.M. 1977, Astr. Ap., 57, 211 .

Cram, L.E., Krikorian, R., and Jefferies, J.T. 1979, Astr. Ap., 71, 14 .

Cram, L.E., and Mullan, D.J. 1979, Ap. J., 234, 579.

Cram, L.E., and Ulmschneider, P. 1978, Astr. Ap., 62, 239.

Cram, L.E., and Woods, D.T. 1982, Ap. J., 257, 269.

Dalgarno, A. 1962, GCA Technical Report No. 62-68-A (Bedford, Mass.).

Delandres, H. 1921, Compt. Rend., 171, 451.

Deutsch, A.J. 1956, Ap. J., 123, 210 .

Deutsch, A.J. 1960, in "Stellar Atmospheres", ed.J.L. Greenstein, Univ. of Chicago Press.

Dumont, S., Heidmann, N., Kuhi, L.V., and Thomas, R.N. 1973, Astr. Ap., 28, 199 .

Dupree, A.K. 1976, in "Physique des Movements dans les Atmosphere Stellaires", eds. R. Cayrel and M. Steinberg (Paris : CNRS).

Durrant, C.J., Grossmann-Doerth, U., and Kneer, F. 1976, Astr. Ap. 51, 95. 
Edmunds, M.G. 1978, Astr. Ap., 64, 103.

Engvold, O., and Rygh, B.O. 1978, Astr. Ap. $\underline{70}, 399$.

Eriksson, K. 1980, Uppsala Astronomical Observatory Report

Feautrier, P. 1964, C. R. Acad. Sci. Paris, 258, 3189.

Fosbury, R.A.E. 1973, Astr. Ap., 27, 129.

Fosbury, R.A.E. 1974, M.N.R.A.S., 169, 147.

Gebbie, K.B., and Steinitz, R. 1973, Solar Phys., 29, 3.

Gebbie, K.B., and Steinitz, R. 1974, Ap. J., 188, 399.

Geltman, S. 1962, Ap.J., 136, 935.

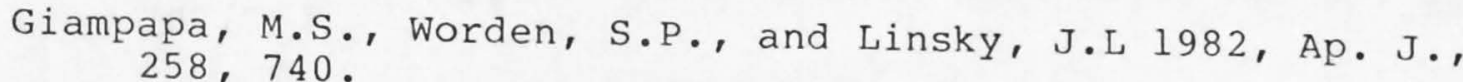

Gingerich, O.J., Latham, D.W., Linsky, J.L. and Kumar, S.S. 1967, S.A.O. Special Report 240.

Gingerich, O.J., and Rich, J.C. 1968, Solar Phys., 3, 82.

Gingerich, O.J., Noyes, R.W., Kalkofen, W. and Cuny, Y. 1971, Solar Phys. $18,347$.

Giovanelli, R.G. 1980, Solar Phys., 68, 49.

Goldberg, L. 1957, Ap. J., 126, 318.

Goldberg, L. 1979, Q.J.R. Astr. Soc., 20, 361.

Golden, L.B., and Sampson, D.H. 1971, Ap. J., 163, 405.

Gray, D.E. 1972, American Institute of Physics Handbook, 3 rd. ed. (McGraw - Hill Book Company).

Gray, D. 1976, The Observation and Analysis of Stellar Photospheres, (John Wiley and Sons, Inc.).

Griffin, R.F. 1968, A Photometric Atlas of the spectrum of Arcturus, Cambridge, England : Cambridge Philosophical Society.

Gustafsson, B., Bell, R.A., Eriksson, K., and Nordlund, A. 1975, Astr. Ap., 42, 407.

Harris, D.L. III 1948, Ap. J., 108, 112.

Heasley, J.N. 1975, Solar Phys., 44, 275.

Heidmann, N., and Thomas, R.N. 1980, Astr. Ap., 87, 36 . 
Hoffleit, D. 1964, Catalogue of Bright Stars, Yale Observatory, New Haven.

Hoyle, F., and Wilson, O.C. 1958, Ap. J., 128, 604.

Inhoff, C.L. 1977, Ap. J., 214, 773.

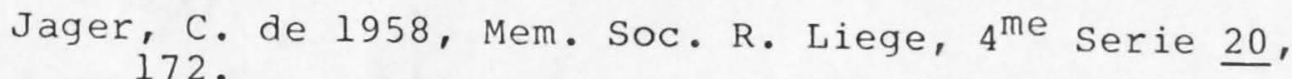

John, T.L. 1964, M.N.R.A.S., 128, 93.

Jefferies, J.T., and Thomas, R.N. 1958, Ap. J., 127, 667.

Jefferies, J.T., and Thomas, R.N. 1959, Ap. J., 129, 401.

Kelch, W.L. 1978, Ap. J., 222, 931.

Kelch, W.L., Linsky, J.L., and Worden, S.P. 1979, Ap. J., 229 ,

Kennedy, P.M. 1981, MK Classification Extension, Centre de donnees Stellaire, Strasbourg III, 19.

Kieffer, L.J., and Dunn, G.H. 1966, Rev. Mod. Phys., 38, 1.

Kneer, F., and Mattig, W. 1978, Astr. Ap., 65, 17.

Kondo, Y. Morgan, T.H., and Modisette, J.L. 1976, Ap. J., 207,167 .

Kraft, R.P. 1959. Ann. Ap., 22, 164 .

Kraft, R.P., Preston, G.W., and Wolff, S.C. 1964, Ap. J., 140,235 .

Kurucz, R.L. 1970, S.A.O. Special Report No. 309.

Kurucz, R.L. 1974, Dudley Obs. Rept., No.9.

Kurucz, R.L. 1979, Ap. J. Suppl., 40, 1.

Linsky, J.L. 1977, in "The Solar Output and its Variation", ed. O.R. White, p477.

Linsky, J.L. 1980, Ann. Rev. Astr. Ap., 18, 439.

Linsky, J.L., and Avrett, E.H. 1970, P.A.S.P., 82, 169.

Linsky, J.L. and Ayres, T.R. 1973, Ap. J., 180, 473.

Linsky, J.L., and Ayres, T.R. 1978, Ap. J., 220, 619.

Linsky, J.L., Bornmann, P.L., Carpenter, K.G., Wing, R.F., Giampapa, M.S., Worden, S.P. and Hege, E.K. 1982, preprint. 
Linsky, J.L., Worden, S.P., MCClintock, W., and Robertson, R.M. 1979, Ap. J. Suppl. $41,47$.

Lo Presto, J.C. 1971, P.A.S.P., 83, 674 .

Lutz, T.E., Furenlid, I., and Lutz, J.H. 1973, Ap. J., 184,
787.

Lutz, T.E., and Pagel, B.E.J. 1978, preprint.

Lutz, T.E., and Pagel, B.E.J. 1982, M.N.R.A.S., 199, 1101. Machado, M.E., and Linsky, J.L. 1975, Solar Phys., 42, 395. Mallia, E.A., and Pagel, B.E.J. 1978, M.N.R.A.S., 184, 55.

Mallik, S.V. 1982, J. Astrophys. Astr., 3, 39.

McMath, R.R., Mohler, O.C., Pierce, A.K., and Goldberg, L. 1956, Ap. J., 124, 1 .

MCClintock, W., Henry, R.C., Moos, H.W., and Linsky, J.L. 1975, Ap. J., 202, 733 .

Menzel, D.H. 1937, Ap. J., 85, 330.

Mihalas, D. 1967a, Ap. J., 149, 169.

Mihalas, D. 1967b, Methods in Computational Physics, 7, 1, Academic Press, New York.

Mihalas, D. 1978, Stellar Atmospheres 2nd. ed. (San Francisco : Freeman).

Milkey, R.W., and Mihalas, D. 1973, Ap. J., 185, 709.

Minnaert, M.G.J., Mulders, G.F.W., and Houtgast, J. 1940, Photometric Atlas of the Solar Spectrum, Utrecht.

Moore, C.E., Minnaert, M.G.J., and Houtgast, J. 1966, The Solar Spectrum $2935 \AA$ to $8770 \AA$, N.B.S. Monograph 61 .

Moos, H.W., Linsky, J.L., Henry, R.C., and McClintock, W. 1974, Ap. J. (Letters). 188, L93.

Mullan, D.J. 1978, Ap. J., 226, 151.

Mullan, D.J., and Cram, L.E. 1982, Astr. Ap., 108, 251.

Neckel, H. 1974, Astr. Ap., 35, 99.

Owocki, S.P., and Auer, L.H., 1980, Ap. J., 241, 448.

Peach, G. 1967, Mem. R.A.S., 71, 1 .

Percival, I.C. 1966, Nucl. Fusion, $\underline{6}, 182$. 
Peytremann, E. 1973, in "Stellar Chromospheres", eds. S.D. Jordan and E.H. Avrett (Nasa SP-317), pl4.

Reimers, A. 1973, Astr. Ap., 24, 79.

Reimers, D. 1975, in "Problems in Stellar Atmospheres and Envelopes", eds. B. Baschek, W.H. Kegel and G. Traving, Springer-Verlag, New York.

Reimers, D. 1977, Astr. Ap., 57, 345 .

Robinson, R.D., Worden, S.P., and Harvey, J.W. 1980, Ap. J. (Letters), 236, L126.

Rodgers, A.W., and Bell, R.A. 1968, M.N.R.A.S., 138, 23.

Sampson, D.H., and Golden, L.B. 1970, Ap. J., 161, 321.

Saraph, H.E. 1970, J. Phys. B. Atom Mol. Phys., 3, 952.

Scharmer, G.B. 1976, Astr. Ap. $\underline{53}, 341$.

Schatzman, E. 1958, Mem. Soc. R. Liege, $4^{\text {me }}$ Serie, 20 , 537 .

Severino, G. 1982, Astr. Ap., 109, 90.

Shine, R.A., and Linsky, J.L. 1974, Solar Phys., 39, 49.

Shine, R.A., Milkey, R.W. and Mihalas, D. 1975, Ap. J., 199, 724 . Shmeleva, O.P., and Syrovatskii, S.I. 1973, Solar Phys., 33 ,
34l.

Smith, M.A., and Dominy, J.F. 1979, Ap. J., 231, 477.

Stapinski, T.E., Rodgers, A.W., and Ellis, M.J. 1978, in "Adv. E.E.P. Symposium on Photo Electronic Image Devices".

Stapinski, T.E., Rodgers, A.W., and Ellis, M.J. 1981, P.A.S.P., 93, 242 .

Stawikowski, A., Glebocki, R. 1978, Astr. Ap., 68, 69.

Stein, R.F. 1981, Ap. J., 246, 966.

Stencel, R.E. 1978, Ap. J. (Letters), 223, L37.

Stencel, R.E., and Mullan, D.J. 1980, Ap. J., 238, 221.

Stenholm, L.G., and Stenflo, J.O. 1977, Astr. Ap., 58, 273.

Sutton, K. 1978, J. Quant. Spectrosc. Rad. Transf., 20, 333.

Taylor, P.O., and Dunn, G.H. 1973, Phys. Rev. \& 8 , 2304. 
Thomas, R.N. 1957, Ap. J., 125, 260.

Thomas, R.N. 1973, Astr. Ap., 29, 297.

Thomas, R.N., and Athay, R.G. 1961, Physics of the Solar Chromosphere (New York : Interscience).

Title, A. 1966, Selected Spectroheliograms (Pasadena: California Institute of Technology).

Ulmschneider, P. 1979, Space Sci. Rev., 24, 71.

Vaughan, A.H., Baliunas, S.L., Middelkoop, F., Hartmann, L.W., Mihalas, D., Noyes, R.W., and Preston, G.W. 1981, Ap. J.',
$250,276$.

Vernazza, J.E., Avrett, E.H., and Loeser, R. 1973, Ap. J., 184,605

Vernazza, J.E., Avrett, E.H., and Loeser, R. 1976, Ap. J. Suppl., 30, 1 .

Vernazza, J.E., Avrett, E.H., and Loeser, R. 1980, Ap. J. Suppl., 45,635 .

Weiler, E.J., and Oegerle, W.R. 1979, Ap. J. Suppl., 39, 537.

Weymann, R. 1962, Ap. J., 136, 844 .

Wiese, W.L., Glennon, B.M., and Smith, M.W. 1969, Atomic Transition Probabilities (Washington : US Government Printing Office) NSRDS-NBS 4 .

Wilson, O.C. 1959, Ap. J., 130, 499.

Wilson, O.C. 1967, P.A.S.P., 79, 46 .

Wilson, O.C. 1976, Ap. J., 205, 823 .

Wilson, O.C. 1978, Ap. J., 226, 379.

Wilson O.C., and Bappu, M.K.V., 1957, Ap. J., 125, 661.

Zelenka, A. 1975, Solar Phys., 40, 39.

Zirker, J.B. 1968, Solar Phys., 3, 164. 